\title{
Lappeenranta
}

University of Technology

Arto Sankala

\section{MODULAR DOUBLE-CASCADE CONVERTER}

Thesis for the degree of Doctor of Science (Technology) to be presented with due permission for public examination and criticism in the Auditorium 1382 at Lappeenranta University of Technology, Lappeenranta, Finland, on the $4^{\text {th }}$ of June, 2015 , at noon.

Acta Universitatis

Lappeenrantaensis 635 
Supervisor

Reviewers

Opponent
Professor Pertti Silventoinen

Laboratory of Applied Electronics

LUT School of Energy Systems

Lappeenranta University of Technology

Finland

Professor Hans Peter Nee

Department of Electrical Energy Conversion KTH Royal Institute of Technology

Sweden

Professor Hirofumi Akagi

Graduate School of Science and Engineering Tokyo Institute of Technology

Japan

Professor Hans Peter Nee

Department of Electrical Energy Conversion

KTH Royal Institute of Technology

Sweden

ISBN 978-952-265-790-9

ISBN 978-952-265-791-6 (PDF)

ISSN-L 1456-4491

ISSN 1456-4491

\section{Lappeenrannan teknillinen yliopisto}

Yliopistopaino 2015 


\section{Abstract}

Arto Sankala

\section{Modular Double-Cascade Converter}

Lappeenranta 2015

160 pages

Acta Universitatis Lappeenrantaensis 635

Dissertation, Lappeenranta University of Technology

ISBN 978-952-265-790-9, ISBN 978-952-265-791-6 (PDF)

ISSN-L 1456-4491, ISSN 1456-4491

Medium-voltage motor drives extend the power rating of $\mathrm{AC}$ motor drives in industrial applications. Multilevel converters are gaining an ever-stronger foothold in this field. This doctoral dissertation introduces a new topology to the family of modular multilevel converters: the modular double-cascade converter.

The modularity of the converter is enabled by the application of multiwinding mediumfrequency isolation transformers. Owing to the innovative transformer link, the converter presents many advantageous properties at a concept level: modularity, high input and output power quality, small footprint, and wide variety of applications, among others. Further, the research demonstrates that the transformer link also plays a key role in the disadvantages of the topology.

An extensive simulation study on the new converter is performed. The focus of the simulation study is on the development of control algorithms and the feasibility of the topology. In particular, the circuit and control concepts used in the grid interface, the coupling configurations of the load inverter, and the transformer link operation are thoroughly investigated. Experimental results provide proof-of-concept results on the operation principle of the converter.

This work concludes a research collaboration project on multilevel converters between LUT and Vacon Plc. The project was active from 2009 until 2014.

Keywords: AC drive, medium-voltage drive, multilevel converter, cascaded H-bridge, mediumfrequency transformer 



\section{Acknowledgments}

The research of my doctoral dissertation was made possible in 2009 when Vacon Plc and Lappeenranta University of Technology joined forces on a new research program. I was lucky to be selected to the group of researchers from the LUT side. I would like to express my sincere gratitude to certain people from Vacon: Dr Hannu Sarén and Mr Risto Komulainen: Your innovative minds created the seed and this work is the fruit.

I am grateful for the effort of the reviewers of this dissertation, Professor Hans Peter Nee and Professor Hirofumi Akagi. Special thanks go to my supervisor Professor Pertti Silventoinen. It was a pleasure to work under your guidance for so many years.

Countless ideas, relentless eagerness to help, support in free time and with professional matters, and friendship - these are the words that describe the atmosphere of our research team, the guys at the office: A big thank you to you all: Dr Juhamatti Korhonen, Mr Janne Hannonen, Mr Tommi Kärkkäinen, Dr Juha-Pekka Ström, Dr Juho Tyster, Dr Mikko Purhonen, and Mr Jari Honkanen.

The quality of this work was greatly improved by the effort of Dr Hanna Niemelä. I thank you for enhancing the grammar and making the work easier to comprehend.

The financial support from Walter Ahlström Foundation and Ulla Tuominen Foundation is highly appreciated. Not a thousand, but 9800 times Thank You.

Mom and Dad, thank you for raising me to become what I am. Thank you for supporting me on my ambitious journey towards the doctoral degree. And Aki, I couldn't have a better brother than you.

Maija, my love and beautiful bride, I'm so lucky and proud to have you. The writing of the dissertation was a challenging task, but it was such a delight to have you next to me in the rough times. I wait eagerly for our fifth anniversary.

Tampere, May 21, 2015

Arto Sankala 



\section{Contents}

$\begin{array}{ll}\text { Abstract } & 3\end{array}$

$\begin{array}{ll}\text { Acknowledgments } & 5\end{array}$

List of Symbols and Abbreviations 9

1 Introduction 15

1.1 Motivation of the work . . . . . . . . . . . . . . 16

1.2 Structure of the MDC . . . . . . . . . . . . . . . . . . 18

1.3 Outline of the work . . . . . . . . . . . . . . . . . . . . . . . . . . . . . . .

1.4 Scientific contributions ....................... 20

2 State-of-the-art of high-power converters 2

2.1 Classic high-power topologies . . . . . . . . . . . . . . . . . 24

2.1.1 Load-commutated inverter . . . . . . . . . . . . . 25

2.1.2 Current source PWM inverter . . . . . . . . . . . . . . 27

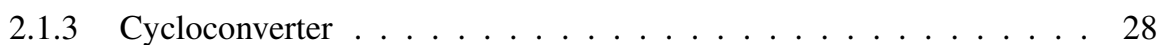

2.2 High-power multilevel converter topologies . . . . . . . . . . . . . 30

2.2.1 Five-level active neutral-point-clamped inverter (5LANPC) . . . . . . 31

2.2.2 Cascaded H-bridge inverter . . . . . . . . . . . . . . . . . 33

2.2 .3 UNIFLEX-PM . . . . . . . . . . . . . . . . 34

2.3 Discussion . . . . . . . . . . . . . . . . . . 36

3 Functional description of the MDC 41

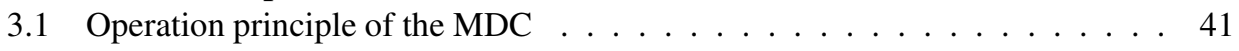

3.2 Selection of the belt voltage rating . . . . . . . . . . . . . . . . . 45

3.3 Multilevel modulation of a cascaded H-bridge inverter . . . . . . . . . . . 47

3.4 Grid interface . . . . . . . . . . . . . . . . . . . . . 50

3.4.1 LCL filter design . . . . . . . . . . . . . . . . . . . . 50

3.4.2 Active front end controller . . . . . . . . . . . . . . 57

3.4.3 Example LCL filter and AFE controller design . . . . . . . . . . . . . 60

3.4.4 Assisted DC link voltage control . . . . . . . . . . . . . . . . . . . 64

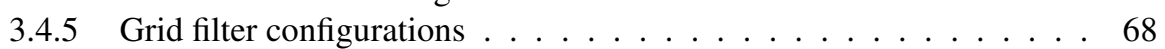

3.5 Load interface . . . . . . . . . . . . . . . . . 71

3.5.1 Motor control: FOC . . . . . . . . . . . . . . . 72

3.5.2 Parallel connection of H-bridges . . . . . . . . . . . . 75 
3.5.3 Delta connection of H-bridges . . . . . . . . . . . . . . . 79

3.5.4 Multiport operation . . . . . . . . . . . . . . . . 81

3.6 Medium-frequency transformer link . . . . . . . . . . . . . . 83

3.6.1 Comparison of transformer bridge cell topologies . . . . . . . . . . . 86

3.6.2 Analysis of the submodule current and voltage frequency spectrum . 93

3.7 Experimental setup . . . . . . . . . . . . . . . . . 101

3.7.1 Measurement results . . . . . . . . . . . . . . 103

4 Feasibility study of the MDC 109

4.1 Comparison of the MDC with the existing topologies in an example application 110

4.2 Performance of the MDC . . . . . . . . . . . . . . . . 115

4.2.1 Transformer voltage stress . . . . . . . . . . . . . . . . 115

4.2.2 Overloading conditions . . . . . . . . . . . . . . . . 119

4.2 .3 Input current quality . . . . . . . . . . . . . . . . . . . . . 122

4.2.4 Asymmetric transformer . . . . . . . . . . . . . . . . . . 128

4.2.5 Seventh winding in the transformer . . . . . . . . . . . . . . . 129

4.2.6 Common-mode voltages and currents . . . . . . . . . . . . 135

4.3 Summary of the feasibility study . . . . . . . . . . . . . . 138

5 Discussion $\quad 141$

6 Conclusion $\quad 145$

$\begin{array}{ll}\text { References } & 147\end{array}$

$\begin{array}{ll}\text { Appendices } & 153\end{array}$

$\begin{array}{lll}\text { A Simulation model } & 154\end{array}$

$\begin{array}{lll}\text { B Simulation parameters } & 158\end{array}$ 


\title{
List of Symbols and Abbreviations
}

\author{
Roman letters \\ C Capacitance \\ $d \quad$ Delay (integer coefficient) \\ E Energy \\ $f \quad$ Frequency \\ G Transfer function \\ $I, i \quad$ Current \\ $i, j \quad$ Index \\ $L \quad$ Inductance \\ L Inductance matrix \\ M Number of cascaded belts or cells \\ M Mutual inductance matrix \\ $N \quad$ Quantity \\ $P, p \quad$ Active power \\ $R \quad$ Resistance \\ $r \quad$ Ratio \\ R Resistance matrix \\ $s \quad$ Laplace transformation variable \\ $t \quad$ Time \\ T Transistor \\ T Torque
}


$U, u \quad$ Voltage

Z Impedance

\section{Greek letters}

$\alpha, \beta \quad$ Axes of the stationary coordinate system

$\eta \quad$ Efficiency

$\Omega \quad$ Rotation speed, mechanical

$\omega \quad$ Angular speed

$\sigma \quad$ Stray impedance

$\varphi \quad$ Angular disposition

\section{Subscripts}

\begin{tabular}{ll} 
aa & Anti-alias filter \\
add & Additional \\
app & Apparent \\
avg & Average \\
circ & Circulating current \\
ctrl & Control \\
d & Damp \\
dc & Intermediate DC link \\
dead & Dead time of the inverter phase leg \\
dis & Distributed \\
em & Electromagnetic \\
eq & Equivalent \\
ext & External \\
$\mathrm{f}$ & Filter \\
$\mathrm{g}$ & Grid \\
$i, j$ & Index \\
inv & Inverter \\
i,o & Input, Output \\
$\mathrm{m}$ & Magnetizing \\
\hline
\end{tabular}




$\begin{array}{ll}\text { mod } & \text { Modulator } \\ \text { mot, m } & \text { Motor } \\ \text { nom, n } & \text { Nominal value } \\ \text { osc } & \text { Oscillation } \\ \text { P } & \text { Primary } \\ \text { r } & \text { Rotor } \\ \text { res } & \text { Resonance } \\ \text { S } & \text { Secondary } \\ \text { s } & \text { Sample } \\ \text { ser } & \text { Series element } \\ \text { sw } & \text { Switch } \\ \text { w } & \text { Winding }\end{array}$

\section{Superscripts}

* $\quad$ Reference value

\section{Acronyms}

2Q Two-quadrant

4Q Four-quadrant

5LANPC Five-level active neutral-point-clamped inverter

$\mathrm{A}, \mathrm{B}, \mathrm{C} \quad$ The three phases of the grid

AC Alternating current

AFE Active front end

CC Current-controlled plant

$\mathrm{CCV}$ Cycloconverter

CHB Cascaded H-bridge inverter

CM Common-mode

CPWM Carrier-based pulse-width modulation

CSI Current source inverter

CSR Current source rectifier

DC Direct current 


\begin{tabular}{|c|c|}
\hline $\mathrm{d}, \mathrm{q}$ & Axes of the rotating coordinate system \\
\hline EMF & Electromotive Force \\
\hline ESR & Equivalent series resistance of an inductor or a capacitor \\
\hline $\mathrm{FC}$ & Flying-capacitor inverter \\
\hline FFT & Fast Fourier Transform \\
\hline FOC & Field-oriented control. \\
\hline GaN & Gallium nitride \\
\hline GCT & Gate-commutated thyristor \\
\hline GTO & Gate turn-off thyristor \\
\hline $\mathrm{HV}$ & High-voltage range, $>20 \mathrm{kV}_{\mathrm{AC}}$ \\
\hline HVDC & High-voltage direct current \\
\hline IGBT & Insulated gate bipolar transistor \\
\hline IGCT & Integrated gate-commutated thyristor \\
\hline IM & Induction motor \\
\hline IP & Intellectual property \\
\hline LCI & Load-commutated inverter \\
\hline LF & Line frequency, 50 or $60 \mathrm{~Hz}$ \\
\hline LL & Line-to-line \\
\hline LN & Line-to-neutral \\
\hline LV & Low-voltage range, $<1.0 \mathrm{kV}_{\mathrm{AC}}$ and $<1.5 \mathrm{kV}_{\mathrm{DC}}$ \\
\hline $\mathrm{M}^{2} \mathrm{C}$ & Modular multilevel converter \\
\hline MDC & Modular double-cascade converter \\
\hline MF & Medium-frequency range, several $\mathrm{kHz}$ \\
\hline MV & Medium-voltage range, $1.0-20 \mathrm{kV}_{\mathrm{AC}}$ \\
\hline NP & Neutral point of the NPC inverter \\
\hline NPC & Neutral-point-clamped inverter \\
\hline PCC & Point of common coupling \\
\hline $\mathrm{PF}$ & Power factor \\
\hline PI & Proportional-integral controller \\
\hline
\end{tabular}


pu Per-unit value

PWM Pulse-width modulation

SHE Selectrive harmonic elimination

SiC Silicon carbide

SM Synchronous machine

TPWM Trapezoidal pulse-width modulation

UPS Uninterruptible power supply

N Neutral

U,V,W The three phases of the load

VC Voltage-controlled plant

VSI Voltage source inverter

ZCS Zero Current Switching 



\section{Chapter 1}

\section{Introduction}

In the field of high-power electric energy conversion, multilevel voltage source inverters (VSI) are an attractive alternative to conventional two-level VSIs and current source inverters (CSI). The two-level converter technology is considered mature owing to its vast application spectrum in low- and medium-power ranges. In the high-power range, however, medium and high voltages are used and the semiconductor technologies are under development. This has given rise to the interest in multilevel converters, which, in addition to a higher voltage quality, use mature low-voltage medium-power semiconductor technologies to achieve high-power ratings of the converter (Franquelo et al., 2008).

The higher power demands of certain applications can be met by parallel connection of multiple two-level converters. Wind power generators in the range of a few megawatts are an example of such an application. The parallel connection of low-voltage two-level converters only increases the power rating of the converter set, but does not have an impact on the voltage rating. As powers increase, higher voltages are used to reduce current and ohmic losses. From this demand, the need for multilevel converters arises (Rodríguez et al., 2002).

Multilevel converters are employed in applications such as compressors, pumps, fans, rolling mills, conveyors, mine hoists, high-voltage direct current (HVDC) transmission, and many more. Because of their acceptance in the industry and intensive research carried out all across the world, the multilevel converters can be regarded as a mature technology. However, not all the potential of the multilevel converters has been implemented by the current technologies. This holds true especially for the energy efficiency, reliability, and power density of the multilevel converters. Therefore, new multilevel converter topologies emerge quite frequently (Kouro et al., 2010).

In the literature, three multilevel converter topologies are considered the classic and mature topologies (Franquelo et al., 2008; Kouro et al., 2010; Najafi and Yatim, 2012). These are a neutral-point-clamped inverter (NPC), a flying capacitor inverter (FC), and a cascaded H-bridge inverter (CHB) (Nabae et al., 1981; Meynard and Foch, 1993; Hammond, 1997). 
These topologies have gained commercial interest in different applications. For instance, NPCs are used in high-power AC motor drives such as conveyors, pumps, and fans, whereas FCs are used in applications that require a high bandwidth (i.e., fast transient recovery) and a high switching frequency such as medium-voltage traction drives. CHBs have been successful in very high-power applications and solutions requiring high power quality. Moreover, there are low-voltage applications of multilevel converters for instance in the solar power industry and in automotive applications.

Each of the classic topologies has a set of special requirements of its own, which affect the design complexity and the control techniques that have to be used. NPC, for example, needs a control approach that balances the voltages of the series-connected DC link capacitors. This is referred to as the neutral point (NP) control in the literature. In FC inverters, again, the voltages in the phase capacitors are naturally balanced, but typically, the required minimum ripple of the voltages of the phase capacitors sets the minimum limit for the switching frequency of the semiconductors. The CHB exhibits no such balancing problems, but instead, it requires hardware that provides isolated DC voltage sources for the series-connected modules.

The classification of multilevel converters can be made according to the application spectrum or by the electrical circuit topology and the semiconductor technology. Figure 1.1 divides the topologies into categories according to the circuit topology and the semiconductor technology. The figure includes topologies that have not been mentioned yet but will be discussed in the literature review in Chapter 2.

\subsection{Motivation of the work}

The different specific requirements of the three classic topologies lead to different applications for each of the topologies. Among the interests of a manufacturer of a multilevel converter is to have a product that has applications in all categories of medium-voltage drives. This means that the topology to be applied should be modular. The client who buys the inverter, on the other hand, may not care about the topology, but the client's interests are in the performance, lifetime costs, reliability of the converter, and the required footprint.

As in any other industry and market, the driving force for a product development comes from the end-users, the customers. In the field of medium-voltage high-power electrical drives, the customer has some kind of a process that needs electrical energy to be converted into mechanical work, or vice versa. The processes in this category include heavy-duty hoists and conveyors, traction motors in trains, megawatt-size fans and pumps that move air or liquid, and wind mills and farms, among others. The installation of power electronic converters in these applications may be a part of a renovation of an old process, an installation of a completely new process line, or retrofitting an old process with converters to improve the system efficiency and controllability.

The customer's interests regarding the power electronic converters in industrial installations 


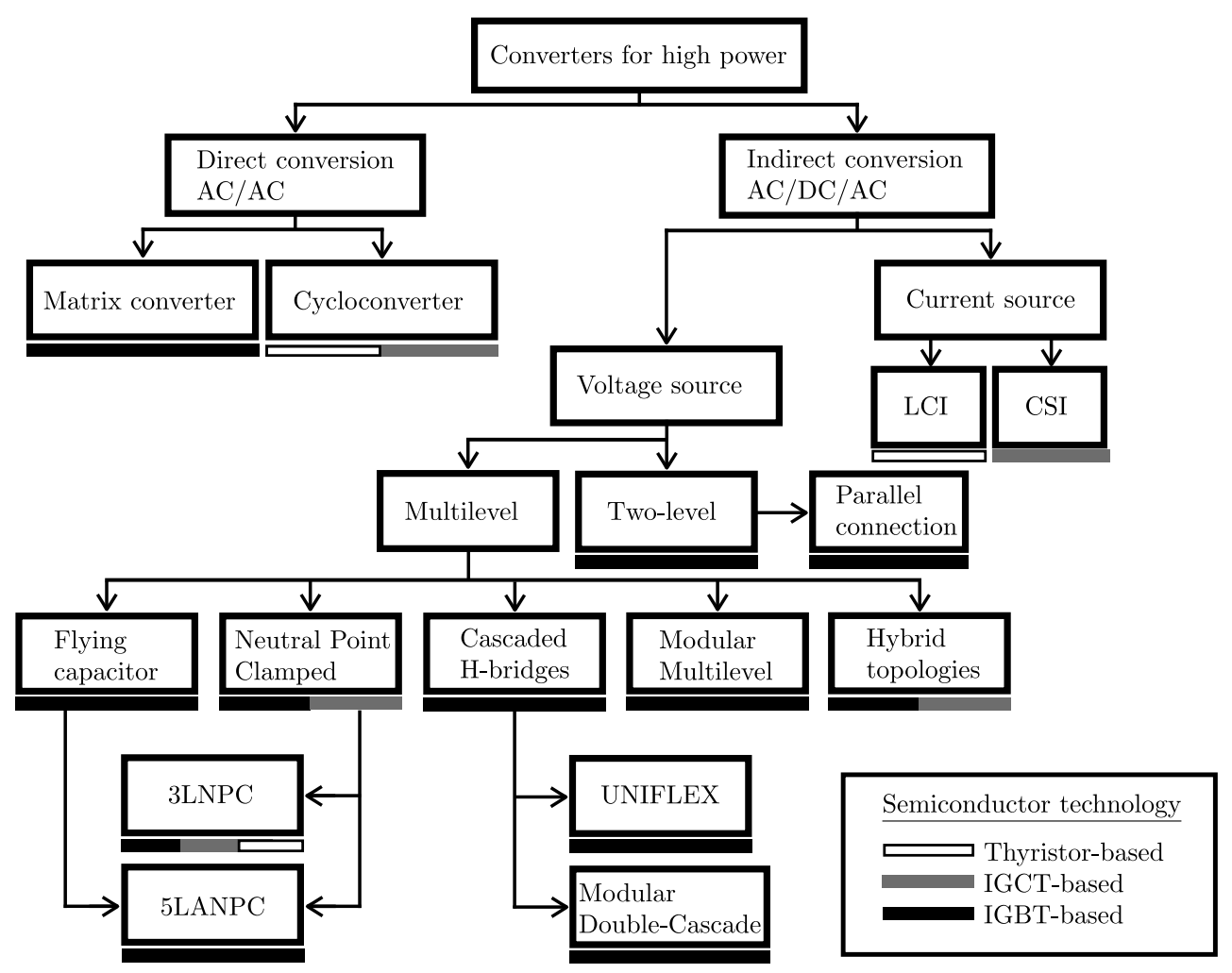

Figure 1.1. Classification of high-power converters according to the circuit topology and the semiconductor technology. Partially reproduced from (Kouro et al., 2010).

can be listed as

1. high efficiency (lower lifetime costs),

2. high reliability (no/few maintenance breaks),

3. low investment costs,

4. simple and adaptive user interface (communications to a higher-level control of the facility),

5. low electromagnetic interference and acoustic noise,

6. small footprint in certain applications (ships, trains, wind mills: smaller size of passive components such as transformers, $\mathrm{d} u / \mathrm{d} t$, and harmonic filters), and

7. low torque ripple (less mechanical stress on mechanical power transmission apparatuses: gears, drive shaft, operating devices),

8. personnel requirements (qualification and training), and

9. product lifetime service and maintenance.

Manufacturers provide solutions to meet the customers' needs. Different customers may have different requirements for instance in terms of voltage, power, and footprint specifications, 
which pose challenges for the manufacturer: In order to meet the specific requirements of multiple customers, many types of converters should be manufactured. Could it be possible to have only one product that is scalable in terms of power and voltage to different applications? Could this product meet simultaneously all or most of the other requirements in the list above?

In order to have a product that is scalable in power, the underlying circuit topology should be modular. In modular topologies, a sufficient number of low-voltage power modules are connected in series or in parallel according to the voltage or current rating of the application. The following questions are relevant to a manufacturer when this type of a medium-voltage product is being planned:

1. What is the multilevel topology that could be provided by this approach?

- The intellectual property (IP) status of the topology should be either free to use or patentable to avoid license fees to the patent owner.

2. Can the manufacturer use existing low-voltage products as the power modules of the medium-voltage drive?

- Manufacturing costs could be low if this is possible

3. Would a customer choose this topology over existing and acknowledged topologies?

At the initiative of Vacon Plc in 2009, LUT and Vacon started a research program, the topic of which was a new modular multilevel converter invented by Mr Risto Komulainen and Dr Hannu Sarén from Vacon. The topology is based on the classic cascaded H-bridge topology featuring one $\mathrm{CHB}$ for the grid connection and another for the load connection. The power module isolation is provided by medium-frequency transformers instead of a linefrequency transformer. First, the functionality of the topology in medium-voltage drives was validated by simulations. The study then continued into the inspection of more specific technical challenges, and finally, a prototype was built to verify some of the simulation-based results. According to the construction, this topology was named a modular double-cascade converter (MDC). The key results of this research program are documented in this doctoral dissertation.

\subsection{Structure of the MDC}

Modular double-cascade converter is a relatively new topology, which belongs to the multilevel voltage source converter family. Vacon Plc owns the US patent, which discloses the invention. The structure and basic building blocks of the converter topology are presented in Figure 1.2 (Komulainen and Sarén, 2013).

The basic building blocks of the MDC are

- Belt is a three-phase three-level unit, which comprises six identical submodules and one medium-frequency six-winding transformer. Connecting multiple belts in series or in parallel increases the voltage rating or current rating of the converter, respectively.

- Filter is installed in the grid interface of the MDC. The preferred structure is an LCL filter. 


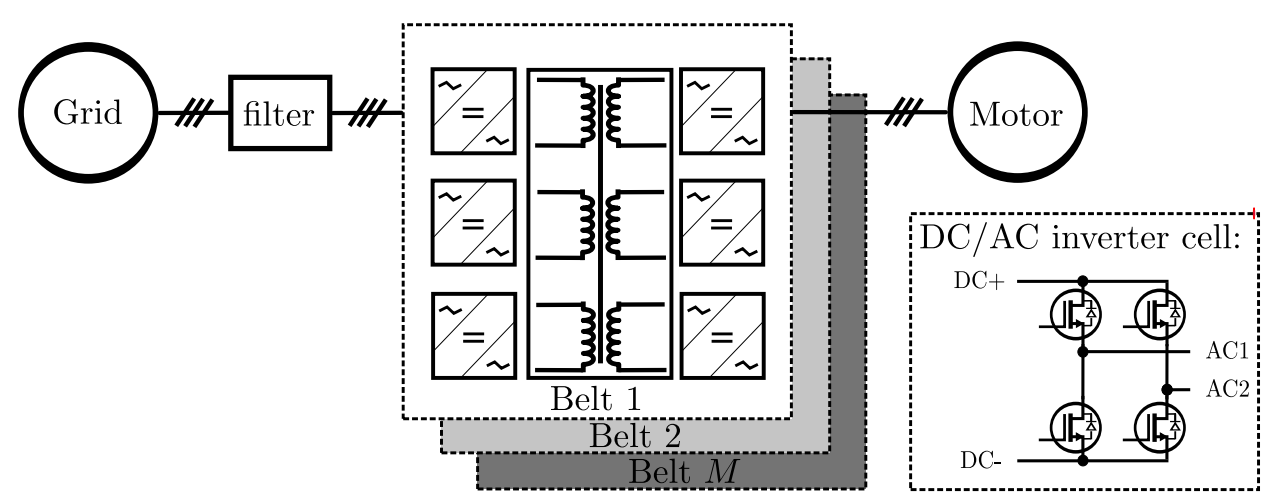

Figure 1.2. Simplified structure of a modular double-cascade converter. The grid and the load are both interfaced with a multilevel cascaded H-bridge inverter.

- Submodule is an AC/AC unit, which comprises two DC/AC inverter cells connected back-to-back and an intermediate DC link capacitor. The first inverter cell is one piece of a cascaded H-bridge (CHB) connection. The second cell interfaces the medium-frequency transformer.

- DC/AC inverter cell is a three-level single-phase unit, which comprises four low-voltage transistors. The type of the bridge cell is widely known as the H-bridge.

- Six-winding medium-frequency transformer is the true innovation of the MDC. The isolation of the submodules required by the CHB setups is provided by the transformer.

The operating principle and features of this converter topology are investigated thoroughly in the following chapters.

\subsection{Outline of the work}

Aside from this introductory part, the rest of the doctoral dissertation is divided into five chapters. Below, a brief description of the contents and applied research methods is given for each chapter.

Chapter 2 A literature review is given of high-power and multilevel topologies that are used in the industry at present. These converter technologies are reviewed in brief in order to establish a realistic view of the technology field and parallel technologies. The chapter is concluded with a comprehensive comparison of the topology-specific features.

Chapter 3 introduces the new multilevel converter topology, the MDC. Various technical details and problems specifically related to the MDC are analyzed and solved. First, the topology is discussed and its characteristic behavior is described. Next, the modulation algorithm and control of the grid inverter and the filter design are presented. Then, the load 
bridge connection methods and the control algorithm are addressed. Further, the details of the medium-frequency transformer link are given. Simulations of the electric and magnetic circuits are made to demonstrate the effectiveness of the proposed control algorithms. The simulations are carried out using Matlab Simulink with the Simscape/SimPowerSystems toolbox. The simulation models employ ideal switching components, and no average modeling of the converters is used. The final part of the chapter provides experimental results, which show the basic principles of the converter operation. The simulations are performed for a full-scale system while the experimental system is downscaled in power and voltage.

Chapter 4 assesses the technical feasibility of the MDC. Here, the topology is compared with parallel technologies with a special focus on the hardware component count. Then, an exhaustive simulation study is performed to explore the limitations of the converter topology and also to propose solutions to the problems discovered in the previous chapter.

Chapter 5 discusses the significance of the research and points out the potential future research subjects.

Chapter 6 concludes the study by summarizing the findings.

\subsection{Scientific contributions}

The scientific contributions of this doctoral dissertation are:

1. Introduction of a new modular multilevel converter topology the applications of which include general motor drives, generator drives, and interconnections of grids

2. Analysis of the control principles needed in the active front end at the grid interface of the converter

3. Analysis of the load bridge connection methods: parallel, star, and delta connections, and multiport operation

4. Analysis of the six-winding medium-frequency transformer link operation

5. Assessment of the feasibility of the converter topology

The author also has other publications on the topic of the work. These publications are listed below but are not appended to the doctoral dissertation.

P1

Sankala, A., Korhonen, J., Ström, J.P., Luukko, J., Silventoinen, P., Komulainen, R., Sarén, H., Södö, N., and Isaksson, D., "Modular double-cascade converter," in Proceedings of the 27th annual IEEE Applied Power Electronics Conference and Exposition (APEC), Feb. 2012, pp. 647-652.

The MDC topology is introduced. Potential applications of the converter are presented, and its capability to change the voltage level is discussed. Simulation results are shown for a 
seven-level version of the converter operating in a $3.3 \mathrm{kV}$ grid with an LR load. The first measurements of a three-level experimental system operating in a $400 \mathrm{~V}$ grid and feeding a motor are reported.

The author's contribution: the principal author of the paper.

\section{P2}

Sankala, A., Korhonen, J., Ström, J.P., Luukko, J., Silventoinen, P., Komulainen, R., Sarén, H., Södö, N., and Isaksson, D., "Modular Double-Cascade Converter for High-Power Medium-Voltage Drives," IET Power Electronics, Accepted for publication on March 25th, 2015

The outline is similar to that of P1. The difference is in the thorough analysis of the mediumfrequency transformer link and more detailed measurement results of the experimental system. The power of the experimental system is increased by a factor of three compared with Publication P1, giving more insight into the dynamics of the converter topology.

The author's contribution: the principal author of the paper.

\section{P3}

Korhonen, J., Sankala, A., Ström, J.P., Luukko J., Silventoinen, P., Komulainen, R., Sarén, H., Södö, N., and Isaksson, D., "Power Direction Control of Medium Frequency Isolation DC/DC Converter for Modular Double Cascade Converter," in Proceedings of the 28th Annual IEEE Applied Power Electronics Conference and Exposition (APEC), Mar. 2013, pp. 2240-2246.

The publication discusses the four-quadrant operation and power flow control principles of the medium-frequency transformer link. Three principles are compared with each other: In the first principle only the primary-side bridges and in the second principle also the secondary-side bridges of the transformer are switched. The third principle uses information from the load bridge controller and measurements to determine whether the corresponding transformer bridge should be switched or not. The third control principle is the preferred one according to the conclusions of the paper.

The author's contribution: the measurement results section of the publication.

\section{P4}

Sankala, A., Korhonen, J., Ström, J.P., Silventoinen, P., Komulainen, R., Sarén, H., Södö, N., and Dilley, D., "Modular Double-Cascade converter with soft switching DC/DC isolation converter," in The 15th European Conference on Power Electronics and Applications, EPE'13 ECCE Europe, Sep. 2013, pp. 1-9.

The application of series resonant circuits to improve the energy efficiency of the mediumfrequency transformer link is introduced. The asymmetry of the transfomer link is found to have a significant effect on the performance of the series resonance. A compensating circuitry is suggested to overcome the asymmetry problem. The simulation results demonstrate the performance of the proposed method.

The author's contribution: the principal author of the paper. 


\section{P5}

Sankala, A., Korhonen, J., Purhonen, M., Ström, J.P., Silventoinen, P., Komulainen, R., Sarén, H., Södö, N., and Strandberg, S., "Design of an Active Front End for a Modular Double-Cascade converter," in The 16th European Conference on Power Electronics and Applications, EPE'14 ECCE Europe, Sep. 2014, pp. 1-10.

The design and implementation of the control of the active front end of the converter are presented. The grid filter and AFE controller design are performed for an example $3.3 \mathrm{kV}$ motor application. The performance of the example design is assessed with simulation results.

The author's contribution: the principal author of the paper.

\section{P6}

Sankala, A., Korhonen, J., Hannonen, J., Ström, J.P., Silventoinen, P., Sarén, H., Komulainen, R., Strandberg, S., and Södö, N., "Flux and Winding Current Balancing Control for a Medium-Frequency Six-Winding Transformer," in The 40th Annual Conference of the IEEE Industrial Electronics Society (IECON), Oct. 2014.

An antisaturation control of the six-winding medium-frequency transformer is introduced. Additionally, the effects of the flux balance controller on the parallel windings on the primary and secondary side are discussed. A control algorithm that both controls the magnetic flux of the transformer and balances the loading of the parallel units is proposed. The algorithm has been granted a Finnish patent in 2013 .

The author's contribution: the principal author of the paper. 


\section{Chapter 2}

\section{State-of-the-art of high-power converters}

The number of multilevel converter topologies is immense, and new topologies emerge quite frequently. The classic topologies and a few new topologies have been commercialized by several manufacturers, and the application spectrum of these converters is wide. This chapter describes the technical background covering circuit topologies and semiconductor technologies, examples of applications, and manufacturer information of selected multilevel topologies. Load-commutated inverters, current-source inverters, and cycloconverters are also described in brief to cover the field of high-power electrical drives to a larger extent.

High-power converters come in the forms of current-source inverters (CSI), load-commutated inverters (LCI), cycloconverters (CCV), two-level voltage source inverters (VSI), and multilevel voltage source inverters. The development of high-power semiconductor devices (up to $8 \mathrm{kV}$ and $6 \mathrm{kA}$ ) extends the power range of CSIs and two-level VSIs. Even though multilevel converters are employed in many industrial applications, they still cannot entirely replace the load-commutated inverters and cycloconverters used in very high-power applications such as large ship propulsion (above $25 \mathrm{MW}$ ) (Rodríguez et al., 2009).

A state-of-the-art analysis of multilevel inverters published by Rodríguez et al. (2002) listed the NPC, FC, and CHB as the three basic topologies that were already commercialized for medium-voltage (MV) applications. The publication discusses the usage of multilevel converters in applications such as: multilevel rectifiers that replace phase-shift transformers and multipulse diode rectifiers, DC/DC converters, nonregenerative and regenerative motor drives, and power systems that require power flow control or harmonic compensation units. The paper delineates future trends of multilevel converters: Some hybrid versions of the classic multilevel topologies are reviewed, applications for the distribution voltage level (11$16 \mathrm{kV}$ ) and distributed energy systems are suggested, and advanced high-power semiconductor technologies such as $3.3 \mathrm{kV}$ and $6.5 \mathrm{kV}$ IGBT technologies are considered as solutions to enable high-power (at least $5 \mathrm{MW}$ ) inverters with a lower number of voltage levels (Rodríguez 
et al., 2002).

A generalized multilevel converter topology was published by F. Z. Peng in 2001. The generalized topology covers all the classic topologies, and it can be modified in many ways to resemble multilevel inverters with different numbers of voltage levels. The basic block of the generalized multilevel converter comprises a voltage source (a capacitor) and a half-bridge consisting of two semiconductor switches. This kind of a building block is used for example in the modular multilevel converter $\left(\mathrm{M}^{2} \mathrm{C}\right)$ (Glinka and Marquardt, 2005). The generalized topology itself is not commercialized.

Less than a decade later, another study on multilevel inverters was published by Franquelo et al. (2008). Again, the same three topologies were presented as the classic topologies. The publication reviewed the applications, modulation techniques, and modeling methods of the most popular topologies. In addition to the applications presented by Rodríguez et al. in 2002, Franquelo et al. discuss applications such as traction drives, automotive, uninterruptible power supplies (UPS), photovoltaic and wind energy applications, magnetic resonance imaging, and high-voltage direct-current (HVDC) applications.

A few interesting applications of multilevel converters are addressed by Rodríguez et al. (2009). A regenerative downhill conveyor in a Chilean mine carries minerals approximately 5800 tons per hour $1.3 \mathrm{~km}$ down a mountainside on a $12.6 \mathrm{~km}$ long track. The electrical system consists of three induction motor drives, $2.5 \mathrm{MW}$ each, with back-to-back NPCs. These NPCs use gate turnoff thyristors (GTO) as power semiconductors. In a marine application, six NPCs are employed on the multimotor system of a tanker. A $6.6 \mathrm{kV}$ supply is generated by four diesel generators, and energy is distributed to two motor systems. Both have an input transformer, NPC active rectifier, and two NPC inverters. One of the inverters drives a 6.15 MW synchronous motor, which is one of the two main propulsion drives. The other NPC inverter drives a $2 \mathrm{MW}$ auxiliary motor. Train traction systems are also mentioned: the Transrapid system, which is a magnetic levitation train (maglev), also uses NPCs in a back-to-back configuration (Rodríguez et al., 2009).

\subsection{Classic high-power topologies}

The high-power and medium-voltage ranges are regarded as $1-50 \mathrm{MW}$ and $2.3-6.6 \mathrm{kV}$ in the industry (Kouro et al., 2010). On the other hand, in (Wu et al., 2008), the mediumvoltage range is defined as $2.3-13.8 \mathrm{kV}$. In this doctoral dissertation, the voltage ranges are set as follows: low-voltage $<1.0 \mathrm{kV}_{\mathrm{AC}}$ (and $<1.5 \mathrm{kV}_{\mathrm{DC}}$ ), medium-voltage $1.0 \mathrm{kV}_{\mathrm{AC}}-20 \mathrm{kV}_{\mathrm{AC}}$, and high-voltage $>20 \mathrm{kV}_{\mathrm{AC}}$. Multilevel converters are not the only solution in this range: two-level VSIs, current source inverters, load-commutated inverters, and direct-conversion variable-frequency drives also fall into this category and should not be forgotten when comparisons of different technological solutions are made.

The two-level VSI is a standard solution in today's low-power and low-voltage (LV) applications, and its properties are well documented. In medium-voltage drives, the two-level 
topology suffers from the need for MV semiconductor technology. The quality of output voltage is inherently low compared with multilevel topologies or direct converters. For these reasons, the 2LVSI has been left outside the analysis in this section.

Although the matrix converter (MC, also called a forced commutated cycloconverter (Neft and Schauder, 1992)) presents numerous advantages such as sinusoidal input current and compact physical size, it has only gained a minor market penetration. Only one manufacturer, the Yaskawa Electric Corporation, is offering this type of converters for both the lowand the medium-voltage ranges (Yaskawa, 2008). The relatively low interest towards this topology by the industry is caused by the inherent disadvantages: limited output-to-input voltage ratio (max. $86 \%$ for sinusoidal modulation), the more complex commutation compared to VSIs, the insufficient reactive power compensation capability, and problems related to operation under unbalanced line voltage conditions (Friedli and Kolar, 2012). In their publication Friedli and Kolar (2012) concluded: "the MC is one of the academically most investigated but industrially least applied converter topology." This converter topology is not analyzed further in the course of this dissertation.

The topologies that are the top competitors of the multilevel technology are PWM currentsource inverters, load-commutated inverters, and cycloconverters (Kouro et al., 2010). The main circuits of the PWM CSI and LCI are similar. The difference between the two is the semiconductors applied: gate turn-off thyristors or integrated gate-commutated thryristors are used in the PWM CSI while the LCI uses thyristors without a self-turn-off capability (Wu et al., 2008). The basic operating principles, applications, and limitations of the LCI, CSI, and $\mathrm{CCV}$ are reviewed in the following three sections, respectively.

\subsubsection{Load-commutated inverter}

LCI drives are used in very high-power applications, where multilevel converters are not applicable. One of the largest variable speed motor drives in the world is a wind tunnel fan owned by NASA. A $101 \mathrm{MW}$ synchronous motor is operated with an LCI drive. In addition to fans and pumps, LCIs are used in ship propulsion and soft starting of synchronous generators (ABB, 2006; Petersson and Frank, 1972).

The LCI is basically a current source inverter having a DC reactor in the intermediate circuit instead of a capacitor found in VSIs. The commutation strategy of the LCI drive is limited to the firing angle control for turn-on and natural commutation for turn-off because normal thyristors are employed. Gate-turn-off thyristors (GTOs) need a circuit for active turn-off, the current rating of which is in the range of one-third of the thyristor anode current (Mohan et al., 2003). GTOs are needed in current source inverters where pulse width modulation techniques are employed to regulate the output current.

The size of the DC link reactor can be massive; for instance, the water-cooled reactor in MEGADRIVE (4.5-72 MW) by ABB takes one of the five cabins needed for the drive. Two cabins contain the grid and motor converters, one is reserved for the drive controller, and the last one houses the water-cooling unit. 


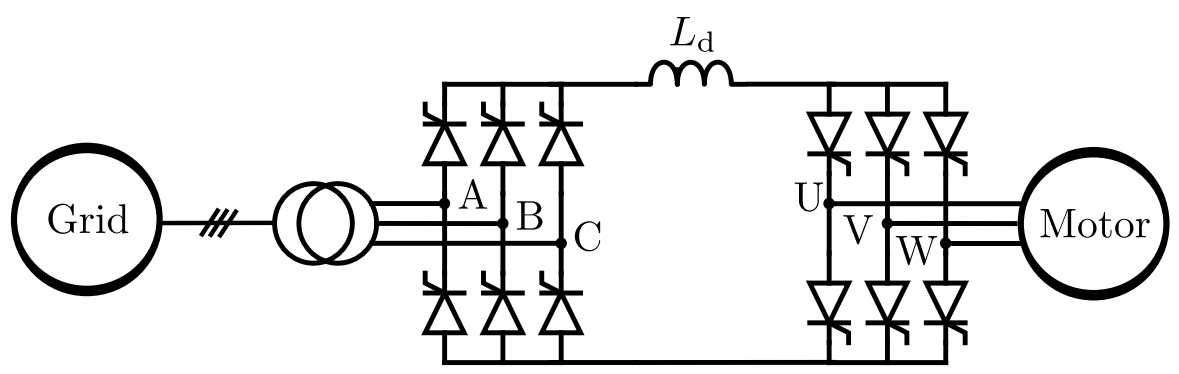

Figure 2.1. Circuit diagram of a load-commutated inverter drive. The converter consists of two identical thyristor bridges and a reactor. The load is normally a synchronous motor.

Features of the LCI that can be regarded as advantageous are the simple converter structure, low switch count, low switching $\mathrm{d} u / \mathrm{d} t$, and reliable overcurrent and short-circuit protection. However, the low dynamic performance as a result of the large DC link reactor and the lack of self-extinguishing capability of thyristors are its drawbacks. The natural commutation of the thyristors occurs by the load voltage with a leading power factor (PF). Therefore, synchronous machines with a leading power factor are the preferred load for the LCI. This limits the application spectrum of the drive (Wu et al., 2008).

The circuit diagram of an LCI drive is presented in Figure 2.1. The thyristor converter connected to the input side rectifies the three-phase input into a DC current, which is filtered by the intermediate circuit reactor $L_{\mathrm{d}}$. The thyristor converter facing the motor load inverts the DC current into three-phase AC currents. Normally, the grid converter operates in the rectifier mode while the load converter operates in the inverter mode. However, for instance in regenerative braking, when the power flow is reversed, the roles of the converters are exchanged (Mohan et al., 2003). The input of the converter may be built with a phase-shifting transformer and series-connected thyristor rectifiers to achieve a 12, 18, or 24-pulse rectification. This will decrease the amount of harmonic distortion produced by the drive to the distribution network voltages (Wu et al., 2008).

The current $I_{\mathrm{d}}$ through the link reactor is controlled by adjusting the firing angle of the grid converter thyristors. The load converter thyristors are naturally commutated with the voltages generated by the back electromotive force (EMF) of the motor. The current $I_{\mathrm{d}}$ in the DC link flows in two phases at a time: when $i_{\mathrm{u}}=I_{\mathrm{d}}$ and $i_{\mathrm{w}}=-I_{\mathrm{d}}$, the current from phase $u$ is shifted to phase $v$ when the back EMF-induced voltage of phase $v$ sets the corresponding thyristors to the forward-biased mode and control current pulses are applied to the gates of those thyristors (Mohan et al., 2003).

It is worth mentioning that the current through the DC link reactor cannot reverse its direction because of the thyristors used in the rectifier and the inverter. Thus, the polarity of the DC link voltage has to be changed when entering the regenerative mode (energy is fed back to the grid from the motor).

The low-speed operation (typically in the range of $10 \%$ of rated speed) of an LCI drive suffers from the low amplitude of the induced back EMF voltage, insufficient to provide turn-off of 
the conducting thyristors. In this operating mode, the current in the DC link reactor must be forced to zero by the rectifier in order to turn off the inverter thyristors (Mohan et al., 2003). When $I_{\mathrm{d}}=0$ A the motor current becomes noncontinuous and results in higher torque ripple produced by the motor. A method to solve the problems caused by this phenomenon is presented in (Petersson and Frank, 1972).

While the output voltages of an LCI drive are almost sinusoidal (except for the spikes produced by thyristor commutations), the currents supplied to the load exhibit a six-step waveform. The stepped waveform includes odd harmonics of the fundamental frequency. The nontriplen odd harmonics cause unwanted deviation in the electromagnetic torque of the machine. The torque ripple results in a deviating rotational speed and/or mechanical stresses experienced by the mechanics connected to the rotor shaft (Wu et al., 2008).

\subsubsection{Current source PWM inverter}

The current source inverter was introduced in the 1970s. The basic circuit is the same as in the load-commutated inverter (Figure 2.1). The original CSI employed thyristors without a self-extinguishing capability, and a special commutation circuit was needed to obtain a sixstep current waveform. Whereas the preferred load of the LCI is a synchronous machine operating at a leading power factor, the CSI is suitable for all kinds of AC motors, especially asynchronous induction motors. Fast response, ruggedness, simplicity of control schemes, regeneration capability, and a wide speed range were the driving forces for the development of this topology (Phillips, 1972).

The CSI did not gain much attention in its early form. Gate turn-off thyristors (GTOs) were used as the main switching component before gate-commutated thyristors (GCT) and integrated gate-commutated thyristors (IGCT) were developed in the late 1990s. With the selfextinguishing capability of these devices, the CSI can employ pulse width modulation (PWM) techniques. The switching frequencies of these devices is very low, and therefore, selective harmonic elimination (SHE) techniques in the PWM operation are often used in the rectifier section of a CSI drive (Wu et al., 2008).

The use of GCTs as the main switching devices eliminates the need for commutation capacitors and diodes, but still, a capacitive filter is needed in the output to provide a current path for the energy stored in the leakage inductance of the machine during switching commutations. Without the filter, high inductive voltage spikes would occur and cause damage to the transistors (Wu et al., 2008).

Similarly to the LCI drive (Figure 2.1), CSIs are normally supplied through an input transformer for common-mode filtering. The transformer has also other functionalities: using a phase-shifting transformer and series-connected six-pulse rectifiers, 12, 18, or 24-pulse rectifier constructions can be established. The harmonic requirements set by the IEEE standard 519-1992 can be met using an 18-pulse configuration. For a given power rating, the 12-pulse configuration is a more cost efficient solution, but it may not meet the harmonic requirements (Wu et al., 2008). 


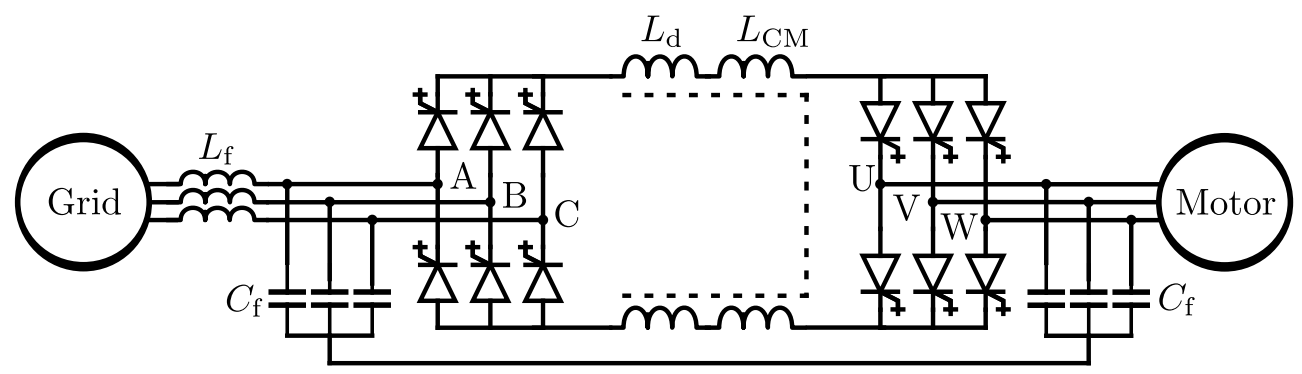

Figure 2.2. Circuit diagram of a transformerless CSI drive with an integrated common-mode DC choke.

The DC link current cannot reverse the polarity in the CSI similarly to the LCI, and in order to supply regenerative energy from the motor to the grid, the polarity of the DC link voltage must be reversed.

An alternative to an input transformer is to have an integrated DC choke serving the functionality of the common-mode filter. This configuration is shown in Figure 2.2. The transformerless grid connection is provided by a PWM current source rectifier (CSR) with a similar structure as the PWM current source inverter. In place of the transformer, an LC filter is used. The neutral points of the grid and the load filter capacitors are coupled to ensure the elimination of common-mode voltage from the stator winding of the motor. The integrated common-mode choke has four windings on the same core: the differential-mode inductance is divided into two parts, and it serves as the main DC link current filter, which is essential for the CSI. The other two windings provide a common-mode inductance, which suppresses the common-mode current. In the example given by Wu et al. (2008), the common-mode inductance is 4.8 per unit (pu) while the differential-mode inductance is $1.0 \mathrm{pu}$. This configuration gives efficient results in the filtering of the common-mode voltages, but the inductance values required are relatively high. The physical size of the DC link reactor can constitute a significant proportion of the footprint of the converter.

As an example, the inverter section of a CSI for a $6.6 \mathrm{kV}$ (line-to-line) network can be built by connecting three GCTs with a voltage rating of $6.5 \mathrm{kV}$ each in series in one inverter branch ( six devices per one inverter phase leg). With a series connection of two of these devices, an inverter for a $4.16 \mathrm{kV}$ utility voltage can be provided. Typical motor applications for these utility voltage levels are on the scale of few megawatts.

\subsubsection{Cycloconverter}

The cycloconverter (CCV) belongs to the direct conversion family of high-power converters. It means that the amplitude, frequency, and phase of the output voltage are directly synthesized from the input phases without a DC intermediate link. This is accomplished with a circuit shown in Figure 2.3. Each of the motor phases is supplied by a converter consisting of two six-pulse thyristor bridges. Each phase module is supplied through an isolation transformer; without the transformer, a short circuit would occur through the neutral point 


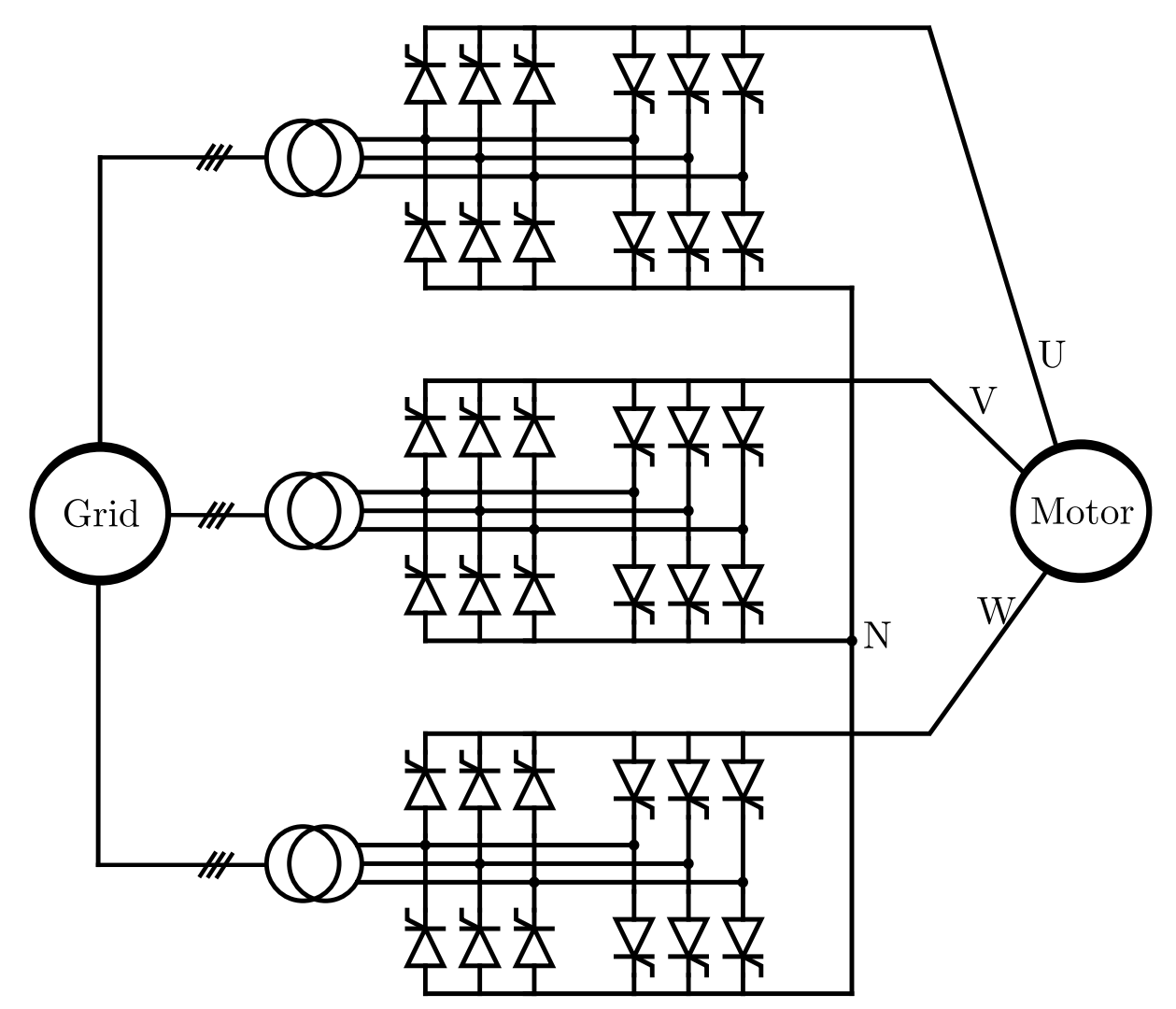

Figure 2.3. Circuit diagram of a cycloconverter. Two six-pulse thyristor bridges and an isolation transformer are needed to supply one motor phase. A common-core transformer can be used for each output phase by having three phase-shifted secondaries wound on the same magnetic core.

connection. The CCV is capable of four-quadrant (4Q) operation because of the back-toback connection of two thyristor bridges per phase.

The output voltage of a cycloconverter is synthesized from input voltages by connecting one of the input phases to the output at a time according to a reference signal. This operation limits the practical output frequency of the $\mathrm{CCV}$ to only a proportion of the supply frequency. However, the converter operates with a high efficiency and a high power, which enables its usage in low-speed high-torque applications such as ship propulsion, grinding mills, and cement mills (Wu et al., 2008).

Wu et al. (2008) highlight one application of a cycloconverter: a grinding mill process for copper production. In this process, four $12.7 \mathrm{MW}$ synchronous motors are operated by four CCVs. The rated voltage (line-to-line) is $1900 \mathrm{~V}$, and the rotor spins nominally at $9.55 \mathrm{rpm}$. This rotation speed is very low, but it is suitable for this application. With 40 pole pairs in the machine, the electrical frequency is $6.4 \mathrm{~Hz}$ which is suitable for a cycloconverter. 
In addition to a low output to input frequency ratio, other drawbacks of the CCV are a low power factor at a low modulation index and the need for filters for harmonic mitigation on the grid side. There is also a possibility that a commutation of a thyristor fails to occur resulting in a short circuit of the motor terminals through the converter. The short circuit results in a very high torque experienced by the machine. Even six times the rated torque may be exceeded (Wu et al., 2008).

In contrast to VSIs, the output voltages of a cycloconverter do not have high $\mathrm{d} u / \mathrm{d} t$ values. The output currents of a cycloconverter are almost sinusoidal, which differs drastically from the waveforms of LCI and CSI converters.

\subsection{High-power multilevel converter topologies}

The amount of academic research effort put into the multilevel converter technology increases annually, and at the same time, the industry has shown a wide interest in the technology. Certain multilevel converter topologies are reviewed in this section. The topologies chosen for the review are five-level active-neutral-point-clamped inverter (5LANPC), the cascaded H-bridge inverter, and the UNIFLEX-PM.

The 5LANPC is chosen for the review because it is an extension of the classic NPC and FC topologies having five levels in the line-to-neutral voltage. The topology has been commercialized by ABB as the ACS2000 product. The classic NPC and FC are omitted from the review because numerous other contributions already provide detailed analyses on them.

Inoue and Akagi (2006) invented a topology for new-generation MV motor drives, which is based on the cascaded H-bridge topology. The converter topology was then adopted to the UNIFLEX-PM project (Universal and Flexible Power Management). UNIFLEX-PM was a EU-funded research program, which took place in the University of Nottingham, UK. Application of the converter was mainly focused on the interconnection of supply networks using the said circuit topology. This converter has a lot in common with and its invention precedes that of the MDC. Therefore, the UNIFLEX-PM system cannot be left unmentioned in the scope of this work. The cascaded H-bridge inverter and the UNIFLEX converter are therefore selected for review here.

The modular multilevel converter is a viable solution for high-voltage direct current (HVDC) applications, but its usability in motor drives is compromised by the poor operation at low output frequencies. Although applications of the $\mathrm{M}^{2} \mathrm{C}$ have recently been reported for motor drives for example in (Spichartz et al., 2013), this topology is not discussed further in this doctoral dissertation.

Modularity has at least two different meanings in the literature. First, the physical construction of a converter can be modular, which means that different operational segments (e.g. rectifier, inverter, control) are separate components. This kind of modularity is advantageous, for instance, if one part gets damaged and has to be replaced quickly. Second, the modularity 
is a property of a multilevel converter that defines its applicability in a wide power and voltage range. The CHB, for instance, is a modular technology since by adding more similar cells in the cascade connection, the voltage and power ratings of the converter can be increased. NPCs and FCs are not modular since the addition of voltage levels changes the circuit design and adds complexity.

The latter definition of modularity is a property that a manufacturer of a multilevel converter appreciates since the same design can be used in a wider application spectrum leading to the need for only one design and potentially lower manufacturing costs.

\subsubsection{Five-level active neutral-point-clamped inverter (5LANPC)}

The 5LANPC converter is an extension of the classic NPC and FC topologies (Bernet and Brückner, 2003; Barbosa et al., 2005). ABB owns the patent to the topology, and the commercial name of the inverter is ACS2000. It is characterized by the following specifications (ABB, 2012).

- Input voltage 4.0-6.9 [kV]

- Output power 250-2500 [kW]

- Output frequency 0-75 [Hz]

- Semiconductor technology: IGBT

The phase legs of the three-level NPC and FC, and the 5LANPC are shown in Figure 2.4. The classic topologies can also be implemented as five-level topologies, but as the number of voltage levels is increased, the NPC requires additional clamping points, and the number of semiconductors needed increases progressively as shown in the equation below. With a three-level $(N=3)$ converter, the number of clamping diodes is two, and there are four switching transistors per phase leg. With five output levels $(N=5)$, the numbers are 12 and 8 , respectively. With an $N$-level NPC converter, the total number of semiconductors is given by

$$
N_{\text {diode }}+N_{\text {sw }}=(N-1)(N-2)+2 N-2=N^{2}-N,
$$

when the blocking voltage of each semiconductor is ideally $u_{\mathrm{dc}} /(2 N-2)$. In addition to the added semiconductors, the number of cascaded capacitors in the DC link is increased. The total number of capacitors is $N_{\mathrm{C}}=N-1$ for the three-phase $\mathrm{N}$-level implementation.

On the other hand, the flying capacitor inverter requires $N_{\mathrm{sw}}=2 N-2$ transistors per phase leg, and the number of flying capacitors per phase leg is given by

$$
N_{\text {cap }}=N^{2}-4 N+4 \text {, }
$$

when the voltage level of each capacitor is ideally $u_{\mathrm{dc}} /(N-1)$ with $u_{\mathrm{dc}}$ representing the total DC link voltage. Here, the number of capacitors is progressively increasing with the number of levels.

The 5LANPC combines the two classic topologies: the clamping diodes of the 3LNPC are removed, and the phase leg of the 3LFC is connected between the emitter of the uppermost 


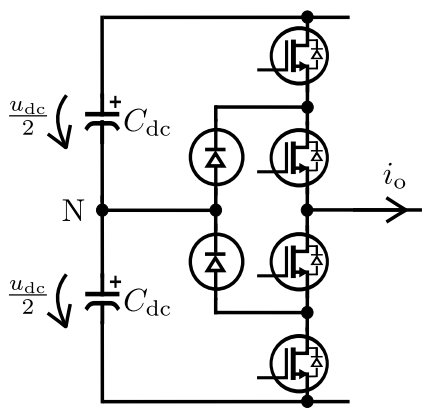

(a) $3 \mathrm{LNPC}$

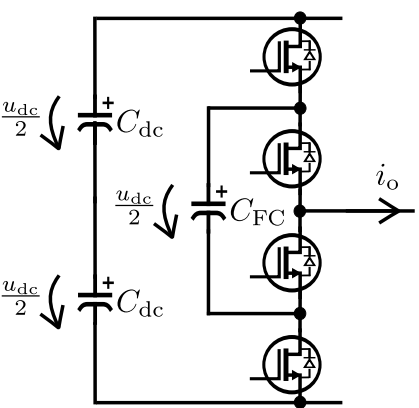

(b) $3 \mathrm{LFC}$

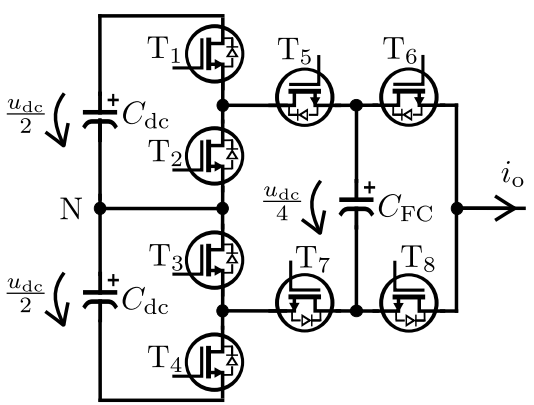

(c) 5LANPC

Figure 2.4. Output phase legs of the classical neutral-point-clamped and flying capacitor inverters, and the active five-level NPC inverter.

transistor and the collector of the lowermost transistor of the NPC leg. While the blocking voltages of each transistor in the NPC and the FC are equal at $u_{\mathrm{dc}} /(2 N-2)$, the transistors in the hybrid 5LANPC are not. Transistors $\mathrm{T}_{1}-\mathrm{T}_{4}$ are rated for $0.5 u_{\mathrm{dc}}$, while the $\mathrm{T}_{5}-\mathrm{T}_{8}$ are rated for $0.25 u_{\mathrm{dc}}$. These voltage-blocking values represent the ideal case where DC link voltage fluctuations are nonexistent and inductive voltage spikes are not produced by current in the stray inductances with high $\mathrm{d} i / \mathrm{d} t$ values during switching operation. In practice, a safety margin should be added to the voltage ratings of the transistors. The nominal voltage level of the flying capacitor is one-fourth of the total DC link voltage.

The 5LANPC can be operated with space-vector PWM, carrier-based PWM, or by selective harmonic elimination PWM methods. A special implementation of the last method is presented in (Kieferndorf et al., 2012). Differently from NPC or CHB, a few switching sequences generated in normal PWM operation are not allowed in the 5LANPC. These switching sequences result in distortion in the output voltage, which is seen as a double commutation: a transition from one level to another without entering the intermediate level. The modulator has two additional tasks not present in a modulator for a CHB inverter: neutral point and flying capacitor voltage balancing. The high number of levels introduces multiple redundant switching states that produce the same output voltage vector, but change the direction of internal current having either an incremental or decremental effect on the voltage of the neutral point. On the other hand, phase voltages have redundancy also making it possible to 
change the direction of the flying capacitor current to either charge or discharge the capacitor (Kieferndorf et al., 2010).

As the 5LANPC topology introduces many positive characteristics to a medium-voltage drive, the topology is still nonmodular. The voltage rating of the converter cannot be increased by connecting the modules in series without input transformers that isolate the power modules. ABB offers this converter with or without input transformer, and the input voltage can be increased by changing the transformation ratio of the input transformer (ABB, 2012). This increase in the input voltage does not provide an increase in the power rating of the converter. The data sheet of the ABB drive suggests that this drive is intended for induction motors. This is a limitation set on the product rather than a limitation set by the topology.

\subsubsection{Cascaded H-bridge inverter}

The cascaded H-bridge inverter was first introduced in the 1970s (McMurray, 1971). The $\mathrm{CHB}$ is one of the three classic multilevel topologies, and it is commercially available from at least seven different manufacturers: Siemens, TMEIC-GE, Arrow-Speed, RXPE, LS Industrial Systems, Yaskawa, and Beijing Leader \& Harvest Electric Technologies (Kouro et al., 2010).

The above-mentioned manufacturers offer CHBs for power levels extending from few megawatts up to $120 \mathrm{MW}$ and voltage levels from $2.3 \mathrm{kV}$ up to $13.8 \mathrm{kV}$. Some of the manufacturers provide the converter with active rectifiers in the submodules while others have diode rectifiers, and the converters are suitable for 2Q operation only.

In order to construct a phase leg circuit of a CHB inverter, a number of H-bridges have to be connected in series as shown in Figure 2.5. All of these bridges have a voltage source capacitor. The capacitors in different cells have to be fed from an isolated voltage source. This is both a pro and a con for the topology: In order to provide the isolation, a complex transformer with multiple secondaries has to be installed, which adds weight, footprint, and cost to the system. On the other hand, the secondary windings of the transformer can be configured to produce phase-shifted outputs canceling low-order harmonics from the input supply current and thereby improving the input power quality and eliminating the need for additional filters.

Similar phase-shifted transformers are often used in cycloconverters, where isolated supply voltages are also needed, and in current source inverters and load-commutated inverters, where the transformers are employed to improve the input power quality and provide common-mode filtering. In the CHB, the transformer is the only nonmodular part.

The semiconductor technology applied to the CHB is the IGBT. The voltage rating of a submodule can be in the LV range, and thus, LV IGBTs can be employed even in MV applications. This is desirable since the low-voltage semiconductor tehchnology is mature, and higher apparent switching frequencies can be obtained through the series connection. 

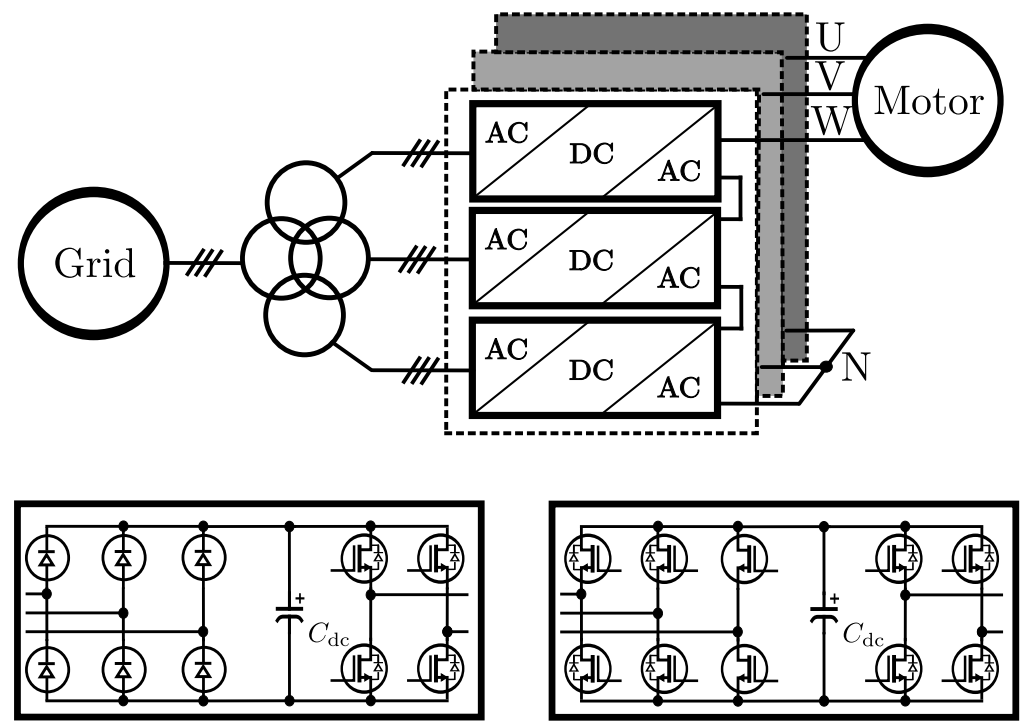

Figure 2.5. Circuit diagram of a cascaded H-bridge inverter. A transformer with multiple three-phase secondaries supplies each submodule. The rectifier can be either a diode bridge (2Q operation) as shown in the bottom left corner, or an active IGBT bridge (4Q operation) as shown in the bottom right corner. Three series-connected H-bridges are depicted on the motor side.

\subsubsection{UNIFLEX-PM}

In order to get rid of the bulky grid-frequency transformer of the CHB inverter, Inoue and Akagi (2006) developed the topology further by adding high-frequency isolating DC/DC converters, which provide isolation to the power cells and enable the use of two CHBs. The first CHB interfaces the utility, while the other is coupled to the load. This topology was introduced to motor drive applications in the next generation. The wide-bandgap transistor technologies such as silicon carbide $(\mathrm{SiC})$ and gallium nitride $(\mathrm{GaN})$ were seen as the enabling technologies efficiencywise for the construction of the high-frequency $(20 \mathrm{kHz})$ transformer link (Inoue and Akagi, 2006, 2007).

This topology was adopted for the Universal and Flexible Power-Management system (UNIFLEX-PM); a research project carried out at the University of Nottingham from 2006 to 2009. The project was funded by the European Union. The topology was intended for use in the interconnection of asynchronous grids. The topology for a three-port application is shown in Figure 2.6. In each of the ports, a CHB converter is applied. The isolation for the H-bridge modules is performed with individual DC/DC converters, which employ medium-frequency isolation transformers. The power cell comprises a back-to-back connection of two IGBT $\mathrm{H}$-bridges with an intermediate DC link and a capacitor. The first $\mathrm{H}$-bridge is connected to the cascade-connection of $\mathrm{H}$-bridges while the other interfaces the two-winding transformer. An experimental setup was constructed at the University of Nottingham, where the operating frequency of the transformers was $2 \mathrm{kHz}$. 


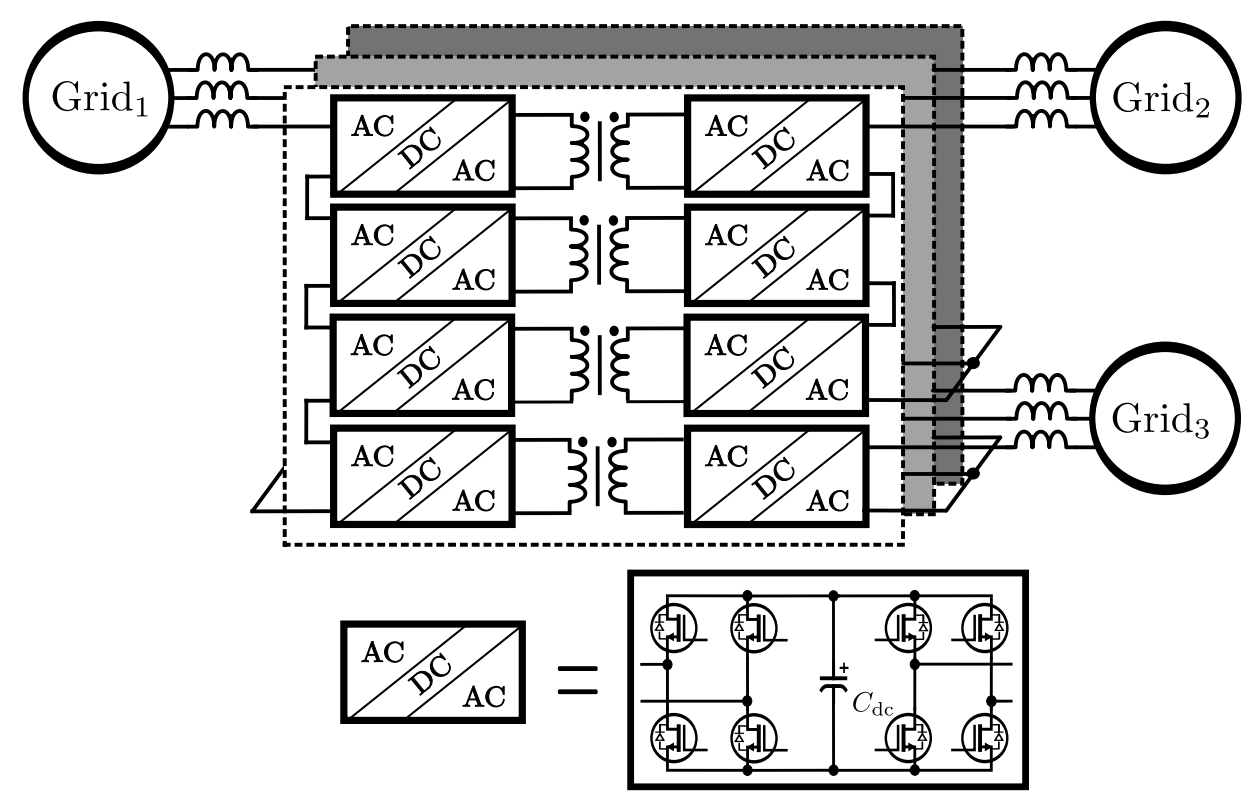

Figure 2.6. Circuit diagram of a UNIFLEX-PM for three-port application. Single-phase mediumfrequency transformers are used to isolate the cells of the cascaded H-bridge connections of each port.

The UNIFLEX-PM system is a flexible energy conversion topology, the basic functions of which include, but are not limited to, the following (Iov et al., 2009):

- Voltage ratio adjustment,

- Interconnection of asynchronous networks,

- Asymmetric current and voltage cancellation,

- Individual control of active and reactive powers,

- Low-voltage ride-through capability, and

- Network support functions such as reactive power injection.

Figure 2.6 shows a three-port application of the topology. A series connection of four Hbridge cells is used in port 1 , which interfaces a medium-voltage network. The second port interfaces another medium-voltage network while port 3 is connected to a low-voltage network. If both medium-voltage networks have the same voltage level, $3.3 \mathrm{kV}$ for instance, one extra cell per phase is applied at port 1 . The same topology can be used for example in a configuration where port 1 is $4.16 \mathrm{kV}$, port 2 is $3.3 \mathrm{kV}$, and port 3 is $690 \mathrm{~V}$ (line-to-line).

One notable issue is the power balance of the ports. Apart from the relatively small amount of energy stored in the DC link capacitors, the topology is not capable of storing energy. Therefore, the powers of the three ports must be balanced, meaning that the sum input power must match the sum output power. Thus, one of the three ports acts as a master in the control of power flow. The internal power balance, that is, the DC link voltage balance, is maintained with an additional control loop. The functionality of this control loop is discussed in detail in Section 3.4.4, where the same method is applied to the MDC. 
The single-phase medium-frequency transformers are operated with a phase-shifted control that enables zero-voltage switching when snubber capacitors are added parallel to each IGBT at the transformer interface (snubbers not shown in Figure 2.6). This control method provides 4Q operation for the converter and is further discussed in (De Doncker et al., 1991; Siemaszko et al., 2009). It is noted here that the transformer construction and the control method are the main differences between the UNIFLEX-PM system and the MDC.

An impact and reliability analysis of the UNIFLEX-PM system found out that, even though the system is complex with many energy conversion stages, the reliability figures are fully comparable with a commercial solution chosen for a reference case. The reference topology was a modular multilevel converter $\left(\mathrm{M}^{2} \mathrm{C}\right)$, which is used for example in HVDC applications. It was stated in the study that special attention has to be paid to the choice of the DC link capacitors: if electrolytic capacitors are used, the reliability will go down, and therefore, the use of polypropylene film capacitors is suggested. The UNIFLEX-PM topology also provides an opportunity to have redundancy in the system. In practice this means that additional cells are included in the cascade connection. This approach is shown to increase the mean time to the first failure by $45 \%$ in the case of 4-out-of-5 redundancy (five cascaded cells used when only four are needed to obtain the full output voltage) (Magro and Savio, 2009).

\subsection{Discussion}

Medium-voltage high-power converters found in the industry at present comprise multilevel voltage source converters, current source converters, and converters with direct conversion. This chapter reviewed a selected few of these topologies.

From the current source family, the PWM-CSI and LCI drives were presented. The major difference between these two is the switching components: while the LCI uses thyristors without a self-extinguishing capability, the CSI uses GTOs and can therefore employ PWM to synthesize the output current. With this capability, the PWM-CSI can be applied to induction motor drives unlike the LCI, which is only suitable for synchronous motors with a leading power factor. Both current source topologies are robust and reliable solutions found in industrial applications.

The cycloconverter (CCV) was selected for review from the direct-conversion family. Unlike the current source topologies, the CCV provides nearly sinusoidal output current to the load. The output voltage is synthesized by successively connecting one of the input phase-voltages to the output thereby imposing a fundamental limitation on the topology: the output frequency is restricted to below approximately one-eight of the input frequency (line frequency).

The three topologies selected from the voltage source family were the five-level activeneutral-point clamped inverter, the cascaded H-bridges, and the UNIFLEX-PM. In these topologies, the voltage in the capacitive voltage sources is synthesized to the output by switching the transistors with selected modulation methods. The 5LANPC and the CHB are direct competitors to the MDC, which is why they were chosen for review. The UNIFLEX- 
PM converter, on the other hand, has a lot in common with the MDC. Its invention precedes that of the MDC, and it was therefore also included in the review.

The main properties of the six topologies considered in this chapter are summarized in Tables 2.1-2.3. In the first table, the input and output waveforms and the converter power ranges are presented. The second table presents the advantages and applications of each topology, and the third table compares the switching frequencies, modulation techniques, and topologyspecific limitations.

Out of the reviewed topologies, the LCI and CCV reach the highest power levels, even up to $100 \mathrm{MW}$. The power range that is of interest in this dissertation is 1-10 MW. Each of the studied topologies have applications in this power range. All of the topologies can be used in 4Q applications. The current source topologies are robust mainly because of the low component count, and reach a very high efficiency. However, their dynamic performances are not at the same level with the voltage source topologies.

The limitation on the dynamic performance is mainly caused by the high-value inductance in the DC link. The current of the motor is adjusted by controlling the current through the DC link inductor. In the voltage source topologies, the DC link energy storage is a capacitor, which acts as a low impedance source when it comes to rapid changes in the output current.

The switching frequencies of the LCI and CCV are the lowest owing to the fact that they employ thyristors without a self-extinguishing capability. The CSI uses GTOs and can thus reach a switching frequency of a few hundred hertz in the specified power range. In the voltage source topologies, the main switching component is the IGBT. Therefore, switching frequencies in the $\mathrm{kHz}$ range are obtained.

Table 2.1. Topology comparison, part 1.

\begin{tabular}{clll}
\hline Topology & \multicolumn{1}{c}{ Voltage } & Current & Power range \\
\hline LCI & $\begin{array}{l}\text { nearly sinusoidal } \\
+ \text { commutation } \\
\text { spikes }\end{array}$ & $\begin{array}{l}\text { quasi-square } \\
\text { wave }\end{array}$ & $\begin{array}{l}\text { tens of MW (typ.), } \\
\text { up to 100 MW }\end{array}$ \\
\hline CSI & nearly sinusoidal & three-level PWM & $\begin{array}{l}<10 \mathrm{MW} \text { (typ.), } \\
\text { parallel connetion }\end{array}$ \\
\hline CCV & $\begin{array}{l}\text { piecewise rep- } \\
\text { resentation of } \\
\text { three-phase input }\end{array}$ & $\begin{array}{l}\text { nearly sinusoidal } \\
\text { +clamping to 0 A } \\
\text { at zero-crossing }\end{array}$ & $\begin{array}{l}<10 \mathrm{MW} \text { (typ.), } \\
\text { up to 70 MVA }\end{array}$ \\
\hline 5LANPC & five-level PWM & nearly sinusoidal & $<10 \mathrm{MW}$ \\
\hline CHB & $N$-level PWM & nearly sinusoidal & $<10 \mathrm{MW}$ (typical) \\
\hline UNIFLEX & $N$-level PWM & nearly sinusoidal & $<5 \mathrm{MW}$ \\
\hline
\end{tabular}


Only the CHB inverter and the UNIFLEX-PM have modular constructions. The basic power modules can be cascaded to increase the voltage level of the converter. In the CHB, the addition of modules leads to the redesign of the only nonmodular part in the converter: the input transformer. The input transformer is replaced by highly modular single-phase DC/DC isolation units inside the UNIFLEX-PM, which increases its modularity, but has a negative impact on the reliability and losses because of the very high component count; 16 transistors plus one medium-frequency transformer per phase per output voltage level. In the CHB, the number of transistors is ten when three-phase input bridges are used for the power cells, and eight when single-phase input bridges are used.

Table 2.2. Topology comparison, part 2.

\begin{tabular}{|c|c|c|}
\hline Topology & Advantages & Applications \\
\hline LCI & $\begin{array}{l}\text { 1. reliable and robust } \\
\text { 2. low manufacturing costs } \\
\text { 3. low switching } \mathrm{d} u / \mathrm{d} t\end{array}$ & $\begin{array}{l}\text { 1. high-power fans } \\
\text { 2. grinding mills } \\
\text { 3. SM starters } \\
\text { 4. Ship propulsion }\end{array}$ \\
\hline CSI & $\begin{array}{l}\text { 1. reliable and robust } \\
\text { 2. very high efficiency } \\
\text { 3. low switching } \mathrm{d} u / \mathrm{d} t\end{array}$ & $\begin{array}{l}\text { 1. fans } \\
\text { 2. pumps }\end{array}$ \\
\hline $\mathrm{CCV}$ & $\begin{array}{l}\text { 1. reliable and robust } \\
\text { 2. low switching losses }\end{array}$ & $\begin{array}{l}\text { 1. low-speed high-torque } \\
\text { 2. ship propulsion } \\
\text { 3. grinding mills }\end{array}$ \\
\hline 5LANPC & high dynamic performance & multipurpose \\
\hline $\mathrm{CHB}$ & $\begin{array}{l}\text { 1. modular } \\
\text { 2. high dynamic performance }\end{array}$ & multipurpose \\
\hline UNIFLEX & $\begin{array}{l}\text { 1. modular } \\
\text { 2. high dynamic performance }\end{array}$ & $\begin{array}{l}\text { grid power } \\
\text { management }\end{array}$ \\
\hline
\end{tabular}


Table 2.3. Topology comparison, part 3.

\begin{tabular}{|c|c|c|c|}
\hline Topology & Switching frequency & Modulation & Specific limitations \\
\hline LCI & $\begin{array}{l}\text { fundamental } \\
\text { frequency }\end{array}$ & $\begin{array}{l}\text { natural } \\
\text { commutation } \\
\text { at turn-off }\end{array}$ & $\begin{array}{l}\text { 1. synchronous } \\
\text { motors with } \\
\text { leading PF, } \\
\text { 2. low dynamic } \\
\text { performance }\end{array}$ \\
\hline CSI & a few hundred $\mathrm{Hz}$ & $\begin{array}{l}\text { SVPWM, SHE- } \\
\text { PWM and TPWM }\end{array}$ & $\begin{array}{l}\text { needs large out- } \\
\text { put capacitors } \\
C \approx 0.3-0.5[\mathrm{pu}]\end{array}$ \\
\hline $\mathrm{CCV}$ & $\begin{array}{l}\text { fundamental } \\
\text { frequency }\end{array}$ & $\begin{array}{l}\text { natural } \\
\text { commutation } \\
\text { at turn-off }\end{array}$ & $\begin{array}{l}\text { 1. needs input } \\
\text { transformer } \\
\text { 2. low output } \\
\text { frequency range } \\
\text { 3. poor PF at low } \\
\text { modulation index }\end{array}$ \\
\hline 5LANPC & $\begin{array}{l}\text { a few } \mathrm{kHz} \\
\text { (apparent) }\end{array}$ & $\begin{array}{l}\text { SVPWM, SHE- } \\
\text { PWM, CPWM }\end{array}$ & $\begin{array}{l}\text { needs NP \& FC } \\
\text { voltage control }\end{array}$ \\
\hline $\mathrm{CHB}$ & $\begin{array}{l}\text { several kHz } \\
\text { (apparent) }\end{array}$ & $\begin{array}{l}\text { SVPWM, SHE- } \\
\text { PWM, CPWM }\end{array}$ & $\begin{array}{l}\text { needs input } \\
\text { transformer }\end{array}$ \\
\hline UNIFLEX & $\begin{array}{l}\text { a few } \mathrm{kHz} \\
\text { (apparent) }\end{array}$ & $\begin{array}{l}\text { SVPWM, SHE- } \\
\text { PWM, CPWM }\end{array}$ & $\begin{array}{l}\text { very high tran- } \\
\text { sistor count } \\
\text { compared with } \\
\text { other topologies }\end{array}$ \\
\hline
\end{tabular}




\section{Chapter 3}

\section{Functional description of the MDC}

The previous chapter dealt with acknowledged topologies for medium-voltage drives. In this chapter, the focus is on a new converter topology. This topology is based on the classic cascaded H-Bridge inverter, where the modularity is increased by replacing the bulky input transformer with medium-frequency transformers. Each transformer has six windings and provides isolation for three submodules on the grid side and three on the load side.

The chapter is organized as follows: First, Section 3.1 introduces the operating principle of the MDC. Section 3.2 presents the dimensioning of power module voltage rating. The multilevel modulation method selected for the MDC is presented in Section 3.3. Section 3.4 addresses the control principles of the grid-side CHB inverter and presents the design guidelines for the grid filter. Section 3.5, again, focuses on the different load connection methods that provide voltage transformation capability. Section 3.6 focuses on the details of the medium-frequency transformer link and control principles. Finally, the chapter is concluded by presenting the measurement results in Section 3.7.

\subsection{Operation principle of the MDC}

In neutral-point-clamped and flying capacitor inverters, the addition of voltage levels leads to changes in the circuit design. In CHBs, on the other hand, it results in the redesign of the input transformer. The MDC is a topology the basic module design of which allows virtually any number of series-connected cells without affecting the circuit design. In practice, the maximum voltage rating for the MDC is set by the isolation strength of the transformer.

The modular structure, a potentially small footprint because of the high-frequency isolation transformer, and a high input and output power quality are factors that motivate the research on the MDC topology. On the other hand, the high switching component count suggests high 
conduction losses, and the high operating frequency of the transformer means high switching losses.

The MDC topology is shown schematically in Figure 3.1. The building block of the converter, a belt, is a three-phase module. The power rating of the converter is defined by the number of belts. If $M$ is the number of belts, the power rating of the converter is $M P_{\text {belt }}$. The voltage rating of the converter can be adjusted according to a specific application by connecting an appropriate number of belts in series or in parallel. A combined series-parallel connection may also be formed.

Each belt comprises six identical submodules and a six-winding transformer, the operating frequency of which is in the range of several kilohertz. The submodule is implemented by a back-to-back connection of two H-bridge cells and a DC link filter capacitor. The first $\mathrm{H}$-bridge cell connects the submodule to the grid or a load, and the other to the mediumfrequency transformer. The H-bridge cells of one belt are not connected in series: the series connection is made between belts.

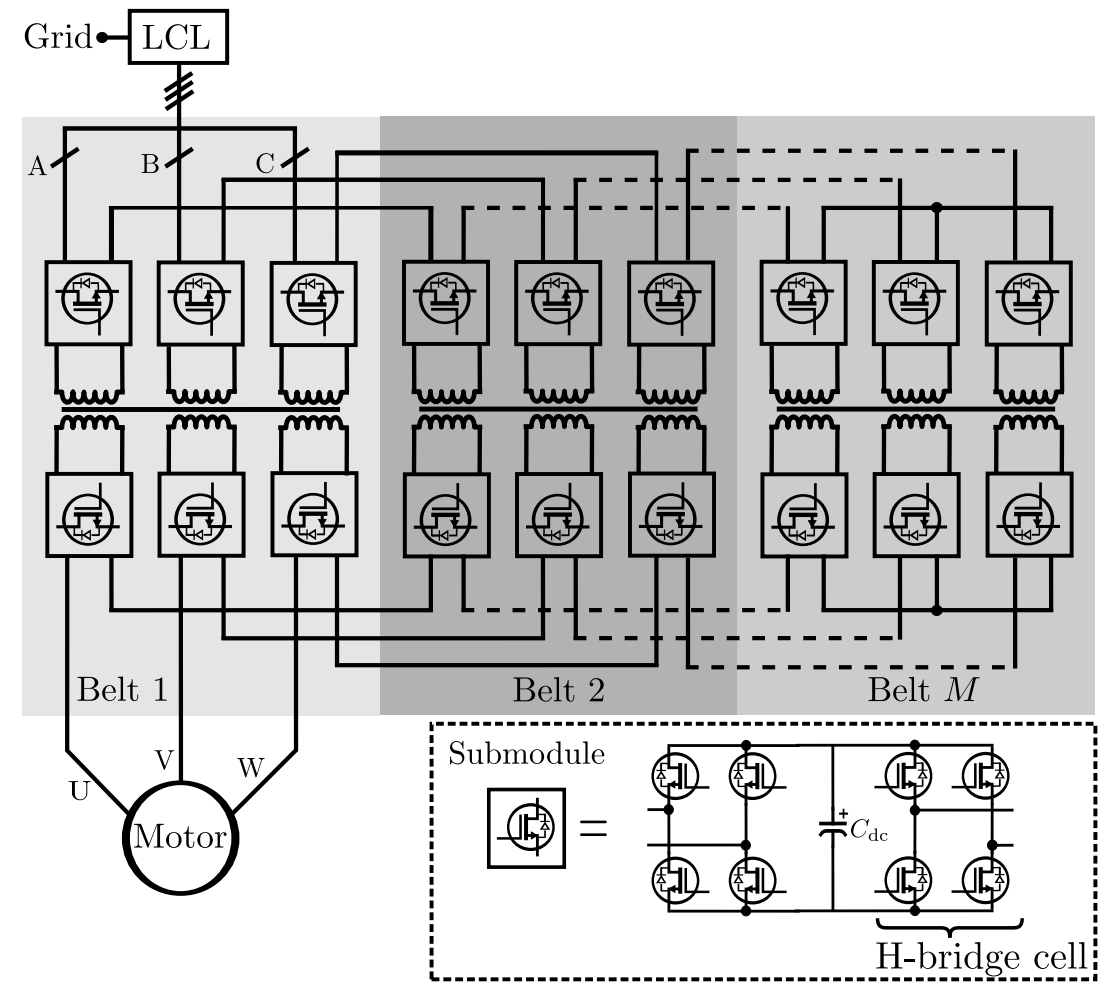

Figure 3.1. Schematic diagram of a modular double-cascade converter. Three-phase power modules, belts, can be connected in series or in parallel. One belt includes a six-winding isolation transformer and six submodules with $\mathrm{H}$-bridge connections to the grid or a load and the transformer. 
The transformer is a symmetric single-phase transformer with six parallel windings. This means that the same amount of magnetic flux flows through each winding at any given time instant. The number of winding turns is the same for each winding, making the transformation ratio equal to one. This type of a transformer can be constructed for instance by using a toroid or an E-core. With a toroidal core, the windings can be distributed with equal spacing around the toroidal form. In the case of an E-core, all the windings are wrapped around the central leg. Symmetric magnetic coupling is provided for the submodules if the winding leakage and wiring impedances are equal among the windings.

The philosophy of the operation can be outlined as follows. Three-phase grid voltage is fed through a grid filter to the converter. A cascaded $\mathrm{H}$-bridge converter rectifies the input voltage to produce multiple DC voltages. The filter capacitors attenuate the high-frequency voltage ripple in the DC links. Inside each belt, three parallel H-bridge cells produce synchronous voltage pulses at a frequency of several $\mathrm{kHz}$ to the three parallel primary windings of the belt transformer. Each grid-connected submodule has a single-phase input, and therefore, the power of one primary-side DC link pulsates at twice the input frequency. The pulsation of the parallel DC links, which are interconnected through the belt transformer, are phase shifted by 120 degrees, and thus, a virtual DC link with a constant instantaneous power is established. On the secondary side of the belt transformer, voltage pulses synchronous to the primaryside switching are induced to three parallel secondary windings. These AC pulses are then rectified to three DC links with active H-bridge cells. These bridges may be switched synchronously with the primary-side bridges, but when the power flow is towards the secondary side, the bridges may function as diode bridges. The DC links on the secondary side include capacitors that again attenuate the high-frequency voltage ripple. The three output H-bridge cells of a belt provide one proportion to the generation of the three-phase output voltage. These H-bridge cells are connected in series or in parallel with the corresponding cells in other belts of the system.

The basic operation of MDC is illustrated using a simulation. The input and output waveforms, transformer winding currents, and DC link voltages in particular are investigated. The simulation is performed with four series-connected belts operating in a $4.16 \mathrm{kV}, 50 \mathrm{~Hz}$ utility and driving a $3.0 \mathrm{MW}, 1486 \mathrm{rpm}$ induction motor. Examination of the simulation results shown in Figure 3.2 reveals the following properties characteristic of the MDC:

1. Input and output voltages have $2 M+1=9$ voltage levels (line-to-neutral);

2. Input and output waveforms are not synchronized;

3. The transformer currents have at least three major frequency components;

- Low-frequency pulsations of the envelopes at twice the fundamental frequencies (the fundamental of the grid in primary phases A, B, C, and the fundamental of the load in secondary phases $\mathrm{U}, \mathrm{V}, \mathrm{W}$ );

- Pulsation generated by the switching operation at the input and output H-bridges, and

- Ripple at the transformer switching frequency and its harmonics.

4. The DC link voltage of a submodule is transferred as an amplitude-modulated signal to the winding current in the same phase. The carrier frequency of this apparent amplitude modulation is the switching frequency of the transformer.

5. The low-frequency pulsation in windings A and $\mathrm{U}$ is nearly in phase opposition, and thus, 
the energy supplied from grid phase A flows mostly into phases $\mathrm{V}$ and $\mathrm{W}$ in the shown time range.

6. The DC link voltages are smaller on the secondary side as a result of the leakage inductance of the transformer.

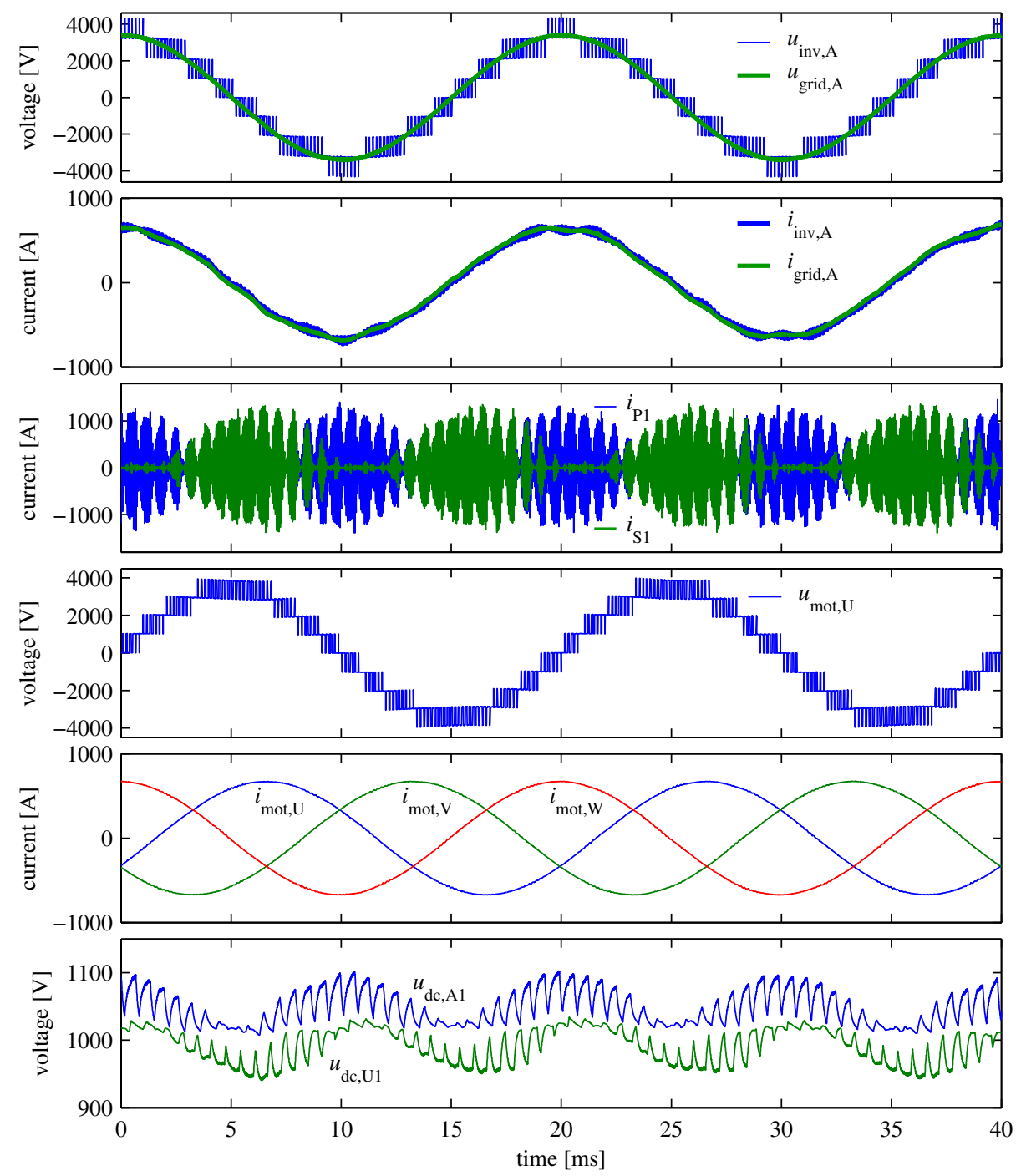

Figure 3.2. Typical waveforms of the MDC. $M=4, U_{\mathrm{LL}}=4.16 \mathrm{kV}, P_{\text {motor }}=3.0 \mathrm{MW}$. From top to bottom: grid voltage and inverter voltage, grid and inverter currents, transformer currents of belt 1 (one primary and one secondary), motor voltage, motor currents, and DC link voltages in phases A and U of belt 1 . 
The doctoral dissertation includes a number of simulations. These simulations are performed with a varying number of belts and various voltage levels. The applied simulation model is presented in Appendix A, and the simulation parameters are given in Appendix B.

\subsection{Selection of the belt voltage rating}

In order to harvest the opportunities of the mature low-voltage IGBT technology, the voltage rating of a belt has to be chosen accordingly. A practical implementation could be achieved with either $1200 \mathrm{~V}$ or $1700 \mathrm{~V}$ IGBT/diode modules. The nominal submodule DC link voltage should be considerably lower than the nominal blocking voltage of the transistors to ensure a sufficient safety margin in the case of DC link voltage fluctuation and inductive voltage spikes caused by high $\mathrm{d} i / \mathrm{d} t$ values during the switching operation. The upper limit of the low voltage range is $1500 \mathrm{~V}_{\mathrm{DC}}$ by definition, but $1700 \mathrm{~V}$ components can still be regarded as low-voltage ones because they are used when the DC link voltage is in the low-voltage range.

Table 3.1 presents the effect of the IGBT/diode module voltage rating on the selection of the submodule DC link voltage and the number of series-connected belts in different grid voltage configurations. A star connection of the cascaded cells is assumed. The number of belts is acquired by dividing the peak line-to-neutral voltage by the nominal DC link value and performing rounding up to the next integer value. The boost ratio $r_{\text {boost }}$ defines the amount of boosting needed by the AFE controller. The boost ratio is calculated by

$$
r_{\mathrm{boost}}=\frac{M u_{\mathrm{dc}, \mathrm{cell}}}{\sqrt{\frac{2}{3}} U_{\mathrm{LL}}} .
$$

It should be noted that for each grid voltage level the construction of a belt remains unchanged. Therefore, the nominal submodule DC link voltages cannot be chosen independently. If the nominal power of a belt is considered constant, the current through the transformer is inversely proportional to the DC link voltage value.

The DC link voltage values deviated slightly from the nominal values $(750 \mathrm{~V}$ and $1100 \mathrm{~V})$

Table 3.1. Comparison of the number of belts and the nominal DC link voltages with different grid voltage levels when $1200 \mathrm{~V}$ and $1700 \mathrm{~V}$ transistors are used.

\begin{tabular}{lllllll}
\multicolumn{3}{c}{$1200 \mathrm{~V}$ modules } & \multicolumn{5}{c}{$1700 \mathrm{~V}$ modules } \\
\hline$U_{\text {LL }}[\mathrm{V}]$ & $u_{\mathrm{dc}, \text { cell }}[\mathrm{V}]$ & $M$ & $r_{\text {boost }}$ & $u_{\mathrm{dc}, \text { cell }}[\mathrm{V}]$ & $M$ & $r_{\text {boost }}$ \\
\hline 1100 & 700 & 2 & 1.56 & 1100 & 1 & 1.22 \\
2300 & 750 & 3 & 1.20 & 1100 & 2 & 1.17 \\
3300 & 750 & 4 & 1.11 & 1100 & 3 & 1.22 \\
4160 & 780 & 5 & 1.15 & 1050 & 4 & 1.24 \\
5700 & 730 & 7 & 1.10 & 1100 & 5 & 1.18 \\
6900 & 770 & 8 & 1.09 & 1050 & 6 & 1.12 \\
\hline
\end{tabular}


Table 3.2. Nominal ampere values for a belt.

\begin{tabular}{lll}
\hline Element & RMS value [A] & Peak value [A] \\
\hline Input current, $\cos \varphi=1.00$ & 420 & 590 \\
Transformer current, primary & 410 & 1320 \\
Transformer current, secondary & 430 & 1490 \\
Output current, $\cos \varphi=0.86$ & 480 & 680 \\
\hline
\end{tabular}

for different grid voltage levels for two reasons: First, the voltage should be approximately constant if the power rating of a belt is considered constant. Second, all voltage levels should be reasonably used. With the $1100 \mathrm{~V}$ grid and $1200 \mathrm{~V}$ modules, one belt is not enough and two are too much to produce the correct voltage. In order to obtain a close to nominal DC link voltage, a very high boosting ratio is needed.

Evaluation of the data presented in Table 3.1 leads to a conclusion that a more practical solution is to choose a submodule DC link voltage in the range of $1000-1200 \mathrm{~V}$ and use $1700 \mathrm{~V}$ IGBTs. As a reference, the $1100 \mathrm{~V}$ DC link voltage was the preferred choice in the UNIFLEX converter also (UNIFLEX-PM, 2007). Moreover, the higher blocking voltage results in a lower total number of switching components.

The nominal power of a belt is chosen as $P_{\text {belt }}=750 \mathrm{~kW}$. For example, the nominal load of the MDC operating in a $4.16 \mathrm{kV}$ grid is $3.0 \mathrm{MW}$. The nominal grid and load current values are defined by

$$
I_{\text {nom }}=\frac{M P_{\text {belt }}}{\sqrt{3} U_{\text {LL }} \cos \varphi}
$$

Thus, the RMS and peak values of the grid and load currents depend on the supply voltage level, not on the DC link voltage. On the other hand, the currents flowing in the windings of the isolation transformers depend on the DC link voltage levels and power. For this reason, the current rating of the transformer is approximately constant at all network supply voltage levels, but the input and output currents of the converter are not.

The nominal currents in different parts of the belt are shown in Table 3.2. The grid and load currents are calculated by (3.2) in the example case of $M=4, U_{\mathrm{LL}}=4,16 \mathrm{kV}$, and $P=3.0 \mathrm{MW}$. In practice, the active power drawn from the grid is higher than the nominal because of losses in the system. The transformer winding current values are obtained from a simulation of this system in the nominal operating conditions (induction motor load).

The data in Table 3.2 reveal a few noteworthy aspects:

1. The RMS value of the current is highest at the load interface because of a lower power factor.

2. The RMS value in the secondary is higher than in the primary as a result of reactive power cycling through the transformer between the output phases.

3. The peak values of transformer currents exceed the grid and load current values by more than two times. 


\subsection{Multilevel modulation of a cascaded H-bridge inverter}

There are numerous pulse width modulation (PWM) methods available for multilevel converters. The methods can be divided into three categories: carrier-based sine triangle comparison, space vector modulation, and optimized pulse patterns such as the selective harmonic elimination method. The desired modulation method depends on the circuit topology of the inverter. For example, the carrier-based level-sifted carriers PWM (LSCPWM) is suitable for neutral-point-clamped and flying capacitor inverters, but not suitable for cascaded H-bridge inverters because of the unequal load sharing among the series-connected cells (Carrara et al., 1992; McGrath and Holmes, 2002; Patel and Hoft, 1973).

Different space-vector- and carrier-based modulation methods for cascaded H-bridge inverters are compared in (Naumanen, 2010). The phase-shifted carriers (PSC) PWM method is a method that results in a balanced loading of the series-connected cells of a CHB inverter. Owing to this property, the PSCPWM method is selected for the simulation study of the MDC. In the following, the basic principles of the PSCPWM are presented and demonstrated by an example.

If $M$ is considered the number of series-connected cells in a CHB configuration, the number of carrier signals in the PSCPWM is either $M$ or $2 M$ depending on the implementation. The phase shift between different carriers is $180^{\circ} / M$ in both of the implementation methods. In the case of $M$ carriers, the reference signal is inverted such that there are two reference signals (noninverted and inverted references). These two methods result in exactly the same switching commands for the CHB. The method with $M$ carriers is used in the simulations.

In a CHB inverter with the PSCPWM, the apparent switching frequency of one H-bridge output voltage is double the switching frequency of one IGBT module. With $M$ seriesconnected cells, the apparent switching frequency of the output voltage is

$$
f_{\mathrm{sw}, \mathrm{app}}=M f_{\mathrm{car}}=2 M f_{\mathrm{sw}, \mathrm{IGBT}}
$$

where $f_{\text {car }}$ is the carrier frequency and $f_{\text {sw,IGBT }}$ the switching frequency of a single IGBT.

A block diagram of the gate pulse generation for one H-bridge cell is shown in Figure 3.3, and an example of the applied PSCPWM method with four cascaded cells is shown in Figure 3.4. The reference voltage and its inverse are compared with the carrier signal of the cell to produce the switching commands for switches 1 and 4 (the upper switch in the positive leg and the lower switch in the negative leg). The negation of these commands gives the commands for switches 2 and 3 (the lower switch in the positive leg and the upper switch in the negative leg). Finally, the dead time that prevents the short circuit of an H-bridge leg is introduced by postponing the gate command transition from zero to one by $d$ modulator sample periods. The delay is calculated by

$$
t_{\text {delay }}=d t_{\mathrm{s}, \bmod }=\operatorname{round}\left(\frac{t_{\mathrm{dead}}}{t_{\mathrm{s}, \bmod }}\right) t_{\mathrm{s}, \bmod },
$$


where $t_{\text {dead }}$ is the desired dead time and $t_{\mathrm{s}, \bmod }$ the modulator sample period. Initially, the reference voltage $u_{i j}^{*}$ is the same for each cell of one phase. $u_{i j}^{*}$ is calculated by

$$
u_{i j}^{*}=u_{i}^{*}=u_{\mathrm{ctrl}, i}^{*} \frac{u_{\mathrm{dc}}^{*}}{u_{\mathrm{dc}, \mathrm{avg}, i}},
$$

where

$$
u_{\mathrm{dc}, \operatorname{avg}, i}=\frac{1}{M} \sum_{j=1}^{M} u_{\mathrm{dc}, i j}
$$

is the average DC link voltage in phase $i$ comprising $M$ series-connected cells. $u_{\mathrm{ctrl}, i}$ is the output of the AFE controller at the grid interface and the motor controller at the motor interface. The index $i \in[\mathrm{A}, \mathrm{B}, \mathrm{C}]$ describes the phase in question and $j \in[1, \ldots, M]$ the cell. The reference voltage is multiplied by the ratio of the reference DC link voltage, $u_{\mathrm{dc}}^{*}$ and the average DC link voltage, $u_{\mathrm{dc}, \text { avg }, i}$, in order to compensate for the DC link voltage variation.
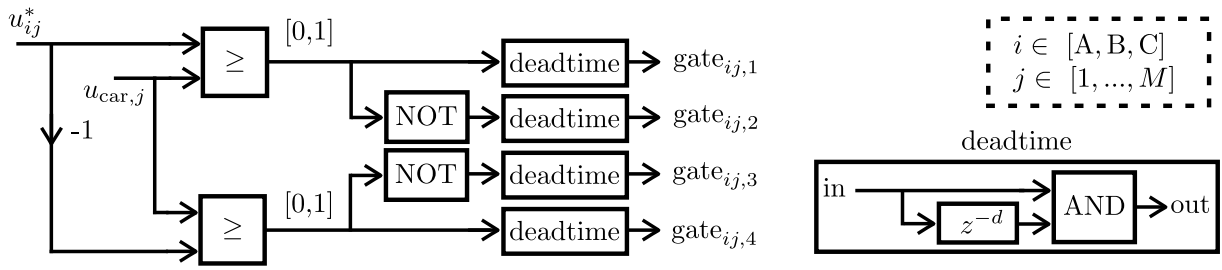

Figure 3.3. Block diagram implementation of the PSCPWM for one H-bridge cell. Similar blocks are needed for each cell of the CHB inverter.
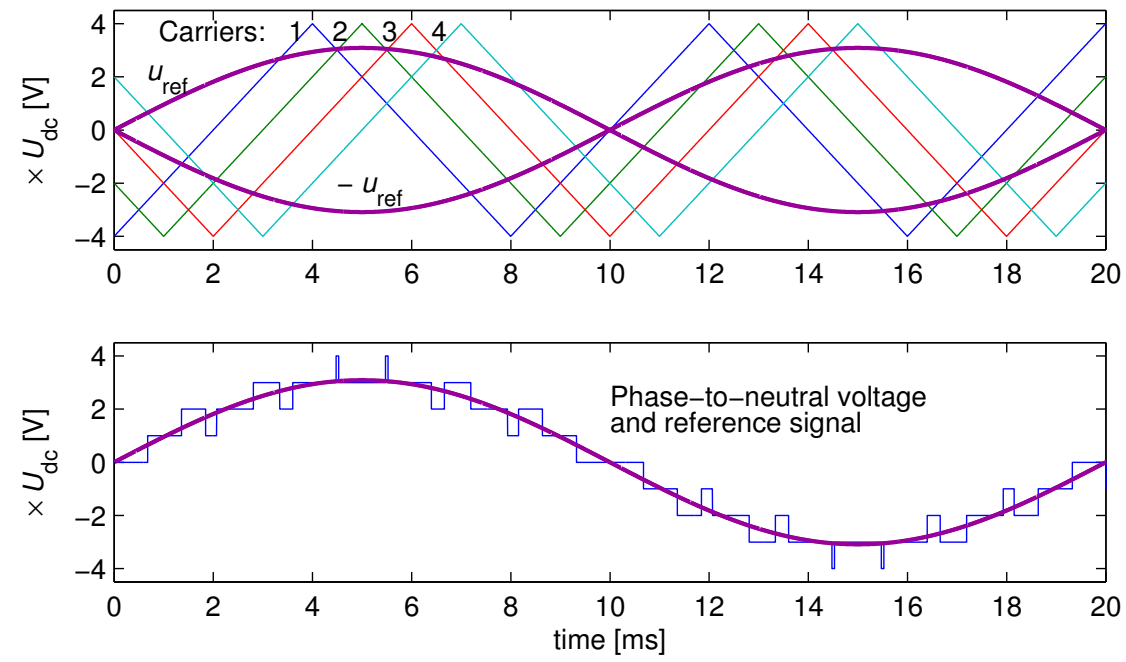

Figure 3.4. Example case of the PSCPWM method when $M=4, f_{\mathrm{sw} \text {,app }}=1 \mathrm{kHz}$, and $f_{\text {ref }}=50 \mathrm{~Hz}$. The number of carriers equals the number of cascaded cells, and the phase difference of adjacent carriers is $\frac{180^{\circ}}{M}=45^{\circ}$. The output voltage has nine levels, and a single IGBT is switched at the $125 \mathrm{~Hz}$ frequency. 
The DC link voltages are filtered by a low-pass filter before the calculation of the average value.

The PSCPWM method was selected for its ability to balance the loading of series-connected cells. Figure 3.5 assesses this property by a simulation in a case of four series-connected belts feeding a motor with $3 \mathrm{MW}$ output power. All DC links in belt 1 are initially at $900 \mathrm{~V}$ while all the other DC links start at $1100 \mathrm{~V}$. At first, a transient occurs when the AFE and the motor controllers initialize, and current builds up at the grid interface. The transient caused by the controller initializations is over in approximately $20 \mathrm{~ms}$. The imbalance of the DC link voltages in belt 1 issues variations in the DC link voltages of other belts also, but when $200 \mathrm{~ms}$ have elapsed, a balanced operation is attained.

The operation of a modulator is impacted by the resolution. The sample time of a modulator $t_{\mathrm{s} \text {, mod }}$ is normally much lower than that of the line current controller $t_{\mathrm{s}, \mathrm{ctrl}}$. $t_{\mathrm{s}, \text { mod }}$ can be the clock period of a DSP or an FPGA in an actual application while $t_{\mathrm{s}, \mathrm{ctrl}}$ normally equals the period of the apparent switching frequency. The triangular carrier signals go through values $-M \ldots M \ldots-M$ during the carrier cycle time $t_{\text {car }}$. The ratio $R=\operatorname{round}\left(\frac{t_{\mathrm{car}}}{t_{\mathrm{s}, \bmod }}\right)$ determines the number of modulator sample cycles during one carrier cycle.

The ratio $R$ is used to determine the resolution of the carrier signal amplitude in the implementation of the modulator. With $M$ series-connected cells, $R$ is first defined as presented in the previous paragraph and then modified such that it is a multiple of $2 M$. This enables the allocation of the $M$ carrier signals to an exactly $180^{\circ} / M$ phase shift. The amplitude of the carriers is $M$, and therefore, the resolution of the carrier amplitudes is $4 M / R$.

As a result of the modification to $R$, the actual apparent switching frequency may be different from the initially determined value. Keeping this in mind, the sample time of the modulator

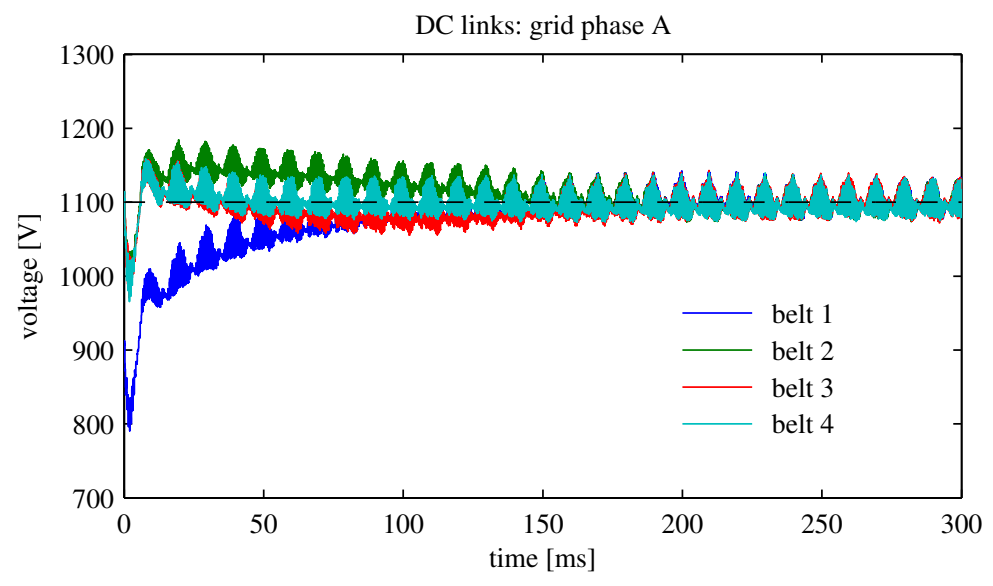

Figure 3.5. Demonstration of the effectiveness of the equal load sharing ability of the PSCPWM on DC link voltages of series-connected cells when $M=4$. The reference DC link voltage of $1100 \mathrm{~V}$ is indicated by a black dashed line. 
and the simulation can be artificially selected such that no modification to $R$ is needed, and the resulting switching frequency equals the initially determined value.

\subsection{Grid interface}

In low-power motor applications, two-level inverters with diode rectifiers are widely used. The diode bridge cripples the performance of the converter in two ways: first, the DC link voltage cannot be regulated, and the voltage level is inversely proportional to the load active power. Second, the converter can only operate in two quadrants of the grid-side voltagecurrent plane: when the motor operates as a generator, for instance in braking of the motor, the energy must be dissipated in a braking resistor to avoid overvoltage conditions in the DC link.

An active front end (AFE) can be implemented if the diode bridge is replaced by an active bridge. In this configuration, the grid filter inductance and the switching of the active bridge increases the DC link voltage and enables the regulation of the DC link voltage and the fourquadrant operation. The latter means that the converter can feed the energy produced by a motor operating as a generator back to the grid. In the MDC, the AFE has to adjust the voltage level such that the voltage drop that occurs over the medium-frequency transformer link is compensated.

An LCL filter is selected instead of a simple L-filter for its better capability to suppress the high-frequency content of the grid current while keeping the filter components relatively small (Liserre et al., 2005).

In the publications on the MDC, the grid is interfaced with diode bridges (Sankala et al., 2012, 2013, 2014a, 2015; Korhonen et al., 2013a). In these publications, the focus is mainly on the medium-frequency transformer link, and the diode bridges are selected because higher DC link voltage fluctuations occur and reveal characteristics that would not be visible when an active front end is used. The benefits and design procedure of an active front end and gridside filter for the MDC are presented originally in (Sankala et al., 2014b). In addition to the previously published material, this section comprises a more thorough analysis of the filter and controller design, presents an assisted DC link voltage controller, and considers different filter configurations which aim at improving the system modularity.

\subsubsection{LCL filter design}

A circuit diagram of a three-phase LCL filter is shown in Figure 3.6. $L_{2}$ and $R_{2}$ constitute the grid-side inductor and its equivalent series resistance (ESR), and $L_{1}$ and $R_{1}$ the inverter-side inductor. The filter capacitors $C_{\mathrm{f}}$ can be connected in a star or delta configuration. $R_{\mathrm{d}}$ is a damping resistor that damps the oscillations in the circuit. The star point of the capacitors can be connected to the neutral point of the grid and/or the neutral point of the CHB inverter. In 


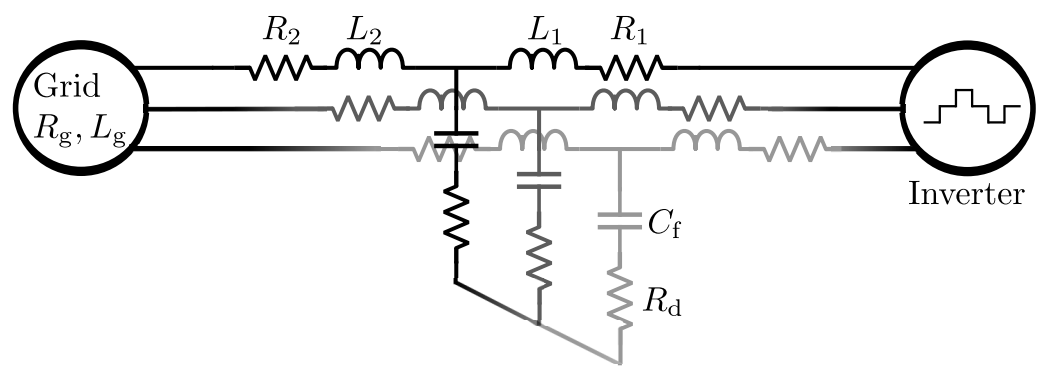

Figure 3.6. Circuit diagram of an LCL grid filter. Grid impedance is denoted by $R_{\mathrm{g}}$ and $L_{\mathrm{g}}$.

the following analysis, the capacitors are star connected, but the star point is not coupled to the neutral point of the grid inverter. Contrary to publication (Sankala et al., 2014b), the index numbers of the filter inductors have been interchanged to harmonize the naming convention with the reference publications.

\section{Grid impedance}

The base quantities of an electrical drive system are defined as

$$
\begin{aligned}
& Z_{\mathrm{b}}=\frac{U_{\mathrm{LL}}^{2}}{S_{\mathrm{n}}} \quad[\Omega] \\
& L_{\mathrm{b}}=\frac{Z_{\mathrm{b}}}{\omega_{\mathrm{g}}} \quad[\mathrm{H}] \\
& C_{\mathrm{b}}=\frac{1}{\omega_{\mathrm{g}} Z_{\mathrm{b}}}[\mathrm{F}] \text {, }
\end{aligned}
$$

where $U_{\mathrm{LL}}$ is the line-to-line voltage of the grid, $S_{\mathrm{n}}$ the nominal apparent power of the drive, and $\omega_{\mathrm{g}}=2 \pi f_{\mathrm{g}}$ the grid frequency. With motor drives, the power factor can be assumed unity in normal operation.

Installations for electric motors usually include a supply transformer that adjusts the voltage levels of the distribution network and the drive. The leakage inductance of the transformer dictates the maximum current in the point of common coupling (PCC). The grid impedance is a sum of resistance and inductance:

$$
Z_{\mathrm{g}}=R_{\mathrm{g}}+j \omega_{\mathrm{g}} L_{\mathrm{g}}
$$

The harmonic content of the grid current is regulated by the standard IEEE 519-1992. The harmonic content limits are defined for different short-circuit to nominal load current ratios, $I_{\text {ratio }}=I_{\mathrm{sc}} I_{\mathrm{n}}^{-1}$. This ratio is a good starting point for the design of the filter: the grid impedance can be determined.

$$
L_{\mathrm{g}}=\frac{Z_{\mathrm{b}}}{I_{\text {ratio }} \omega_{\mathrm{g}}} \sqrt{1-x^{2}}
$$


Equation (3.11) is in a designer-friendly form since it has simple parameters: the base impedance of the electrical system $Z_{\mathrm{b}}$, the short-circuit to nominal current ratio $I_{\text {ratio }}$, and the ratio of grid resistance and impedance $x=\frac{R_{\mathrm{g}}}{\left|\boldsymbol{Z}_{\mathrm{g}}\right|}$.

\section{Inverter-side inductor}

The grid- and inverter-side inductors differ significantly from each other. While the grid-side inductor is a line-frequency reactor, the inverter-side inductor has to withstand ripple current at the apparent switching frequency of the inverter. Thus, the core material of the inverterside inductor depends on the frequency and the cross-sectional area of the core depends on the maximum ripple of the current. The ripple current, on the other hand, is normally selected such that the current rating of the semiconductor switches is not exceeded (Rockhill et al., 2011).

Yet another limiting factor for the inductance of the inverter-side inductor comes from thermal limitations. An excessive current at the switching frequency may cause a considerable temperature rise in the core of the inductor because of the higher hysteresis losses and eddy currents. For the simulation study of the MDC, no real-world values for the current ripple specification can be obtained. Therefore, a generic value of $10 \%$ of the nominal current amplitude is used to determine the inductance.

When choosing the current specifications for the MDC, it is noted that one of the greatest benefits of the MDC is the use of similar power electronic building blocks in all H-bridge cells. Thus, the same IGBT component can be used at the motor and transformer interfaces as at the grid interface. The motor bridge switches normally carry a higher current than the grid bridge switches because of the lower power factor. It is typical for the MDC that the transformer bridge switches experience repetitive peak currents more than twice the peak current of the grid or motor bridges. Therefore, the choice of a 10\% ripple current for the inverter-side inductor and the grid bridge IGBTs is an artificial limit that is not determined by the actual current rating of the IGBTs in the grid-side H-bridges.

The design procedure of an LCL filter for the MDC was presented in (Sankala et al., 2014b). The applied design guidelines were published by Liserre et al. (2005), but a new dimensioning method for the inverter-side inductor was proposed. The proposed method is based on the frequency domain analysis of the inverter output voltage in the nominal operating point. The values of the voltage components in the vicinity of the switching frequency were calculated, and the sum impedance of the inverter-side inductor, the capacitor, and the damping resistor was tuned such that the switching frequency component of the phase current was at a desired level. The proposed method was deemed too complicated compared with other published methods.

For instance, the method published by Rockhill et al. (2011) defines the maximum volt second deviation during a switching cycle as a function of modulation index $m$ and time instant during a fundamental cycle. With the 3LNPC and the space vector pulse width modulation 
(SVPWM), the maximum deviation occurs when $m=1 / \sqrt{3}$ and the angle of the reference voltage vector equals $90^{\circ}, 210^{\circ}$, or $330^{\circ}$ (equal to the time instants when the phase voltages cross zero).

The maximum volt second deviation in the case of a $2 M+1$-level $\mathrm{CHB}$ inverter with the PSCPWM was calculated as a function of modulation index and number of series-connected cells. The procedure for the calculation starts by defining the reference voltage in discrete representation by

$$
u^{*}\left(k t_{\mathrm{s}}\right)=m M \sin \left(2 \pi f_{\text {grid }} k t_{\mathrm{s}}\right), k \in\left[0,1, \ldots \operatorname{round}\left(\frac{1}{f_{\text {grid }} t_{\mathrm{s}}}\right)\right],
$$

where $t_{\mathrm{s}}=\frac{10^{-6}}{6} \mathrm{~s}$ is the applied sample time. The output voltage of the grid inverter was defined as shown in Section 3.3. Then, the flux linkage (volt seconds) of the inverter-side inductor was determined from the difference of the inverter output voltage and the reference voltage

$$
\psi_{L_{1}}\left(k t_{\mathrm{S}}\right)=\psi_{L_{1}}\left((k-1) t_{\mathrm{s}}\right)+u_{\mathrm{dc}, \text { cell }} \times\left(u_{\mathrm{inv}}\left(k t_{\mathrm{s}}\right)-u^{*}\left(k t_{\mathrm{s}}\right)\right) t_{\mathrm{s}} .
$$

Calculating the difference of the voltages eliminates the fundamental wave from the inverter output voltage. Next, the sideband components of the switching frequency and their harmonics define the $\psi_{L_{1}}$. The maximum deviation of flux linkage during a switching cycle over the fundamental frequency cycle was then defined in order to calculate the minimum inductance at which the maximum allowed current ripple occurs.

The modulation index $m$ was varied from 0 to 1 and the number of cells $M$ from 1 to 6 . Other parameters of the calculation are shown in Table 3.3. The nominal power of one belt is $P_{\text {belt }}=750 \mathrm{~kW}$ and the maximum current ripple is defined as

$$
\Delta I_{L_{1}, \max }=0.1 \times \frac{\sqrt{2} P_{\mathrm{n}}}{\sqrt{3} U_{\mathrm{LL}}}=0.1 \times \frac{\sqrt{2} M P_{\mathrm{belt}}}{\sqrt{3} U_{\mathrm{LL}}} .
$$

The flux linkage deviation is illustrated in Figure 3.7. The figure shows that when $m>$ $0.5 \mathrm{M}^{-1}$, the maximum flux linkage deviation is approximately constant. The DC link voltage has a clear effect on the results: with $M=4$ and $M=6$, the deviation is less than in other

Table 3.3. Parameters used in the calculation of the maximum flux linkage deviation of the inverter-side inductor.

\begin{tabular}{llll}
\hline$M$ & $U_{\mathrm{LL}}[\mathrm{V}]$ & $u_{\mathrm{dc}, \text { cell }}[\mathrm{V}]$ & $\Delta I_{L_{1}, \max }[\mathrm{A}]$ \\
\hline 1 & 1100 & 1100 & 55.7 \\
2 & 2300 & 1100 & 53.2 \\
3 & 3300 & 1100 & 55.7 \\
4 & 4160 & 1050 & 58.9 \\
5 & 5700 & 1100 & 53.7 \\
6 & 6900 & 1050 & 53.2 \\
\hline
\end{tabular}




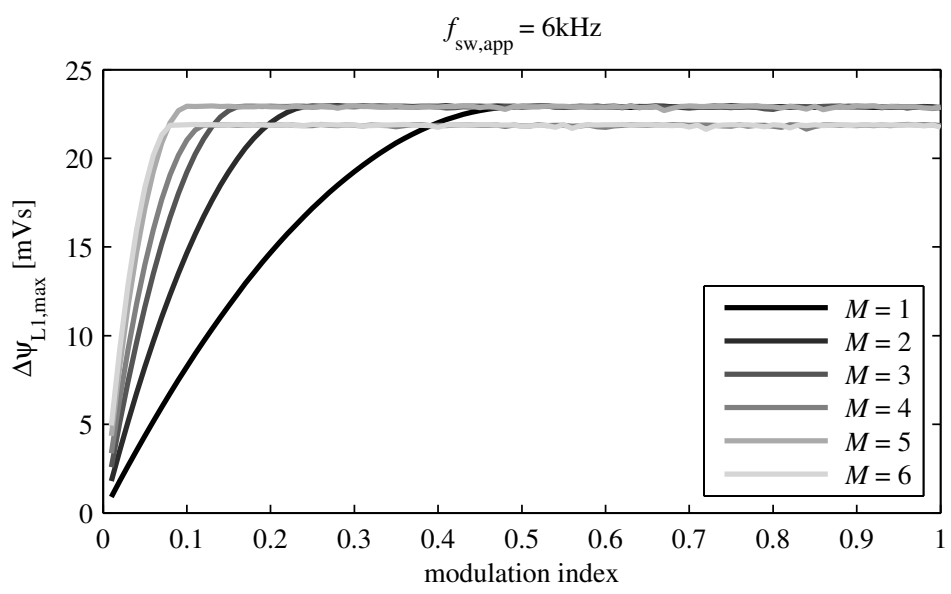

Figure 3.7. Flux linkage deviation of the inverter-side inductor as a function of modulation index and number of series-connected cells.

cases because the DC link voltage is $50 \mathrm{~V}$ less. Further, the number of belts has no effect on the volt seconds if the apparent switching frequency is constant.

Knowing that the maximum flux linkage occurs when $m>0.5 \mathrm{M}^{-1}$, the highest deviation is produced whenever the reference voltage is halfway between two voltage levels; see Figure 3.8. Assuming a constant reference voltage of $0.5 M^{-1}$ over one switching cycle, the maximum volt seconds can be defined analytically

$$
\begin{aligned}
\Delta \psi_{L_{1}, \max } & =\psi_{L_{1}, \min }+\left.u_{\mathrm{dc}, \text { cell }} \int_{0}^{0.5 t_{\mathrm{sw}, \text { app }}}\left(u_{\mathrm{inv}}(t)-u^{*}(t)\right) d t\right|_{\Delta \psi_{L_{1}, \max }=-\psi_{L_{1}, \min }} \\
& =\frac{u_{\mathrm{dc}, \mathrm{cell}}}{8} t_{\mathrm{sw}, \mathrm{app}} .
\end{aligned}
$$

Thus, the minimum inductance needed to suppress the current ripple below the value $\Delta I_{L_{1}}$,max is defined as

$$
L_{1, \min }=\frac{\Delta \psi_{L_{1}, \max }}{\Delta I_{L_{1}, \max }}=\frac{u_{\mathrm{dc}, \text { cell }}}{8 f_{\mathrm{sw}, \text { app }} \Delta I_{L_{1}, \max }} .
$$

Assuming parameters from Table 3.3 with $M=4$ and $f_{\mathrm{sw} \text {,app }}=6 \mathrm{kHz}$, the maximum flux linkage deviation is $21.9 \mathrm{mVs}$ according to (3.16) and the minimum inductance is $371 \mu \mathrm{H}$ according to (3.17). The value $21.9 \mathrm{mVs}$ correponds well with the maximum volt seconds shown in Figure 3.7 for $M=4$ and $M=6$.

\section{Filter capacitor}

After the dimensioning of the filter inductors, the filter capacitor and the damping resistor have to be determined. According to Liserre et. al. (2005), it is practical to choose a capacitor 


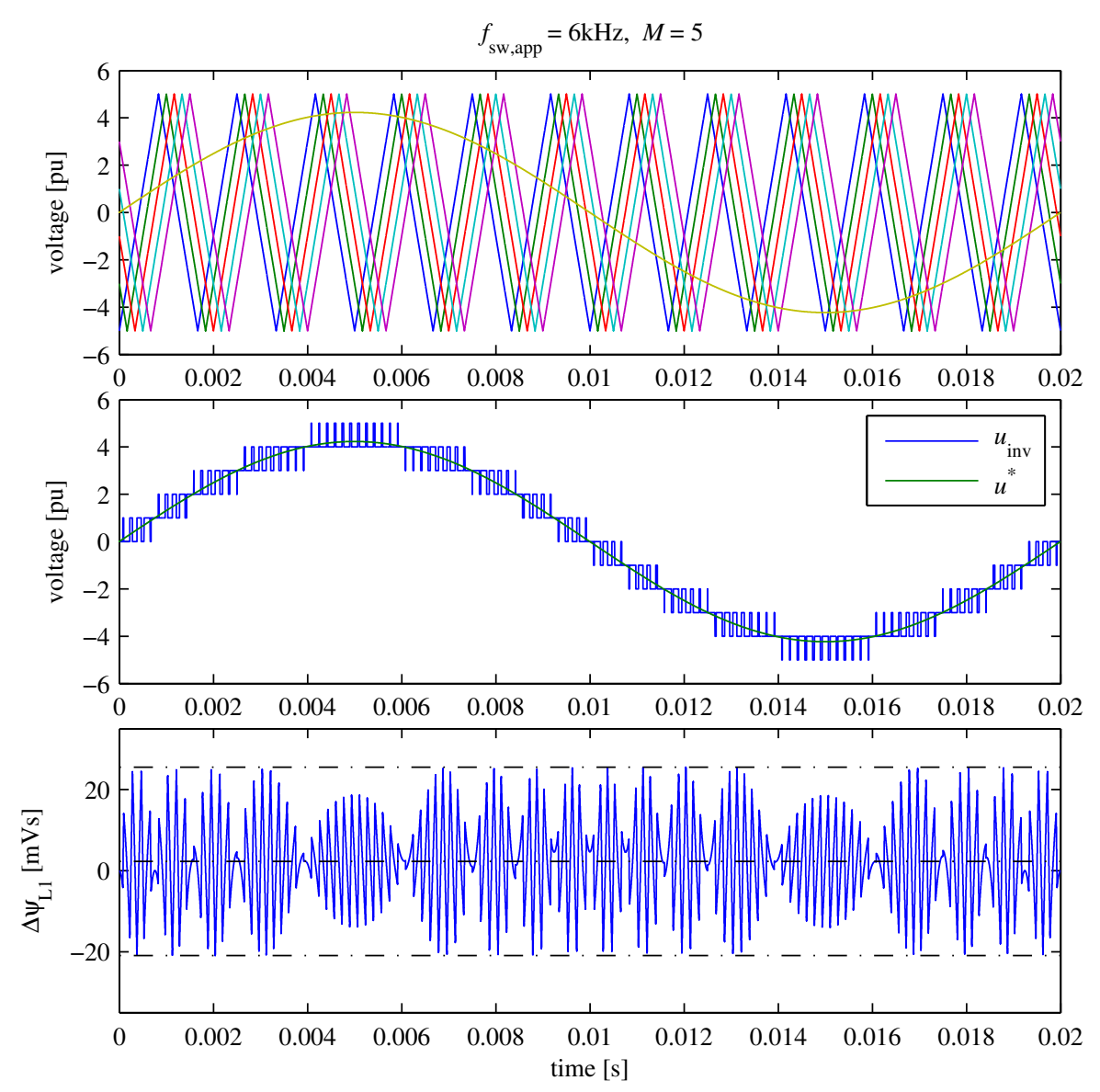

Figure 3.8. Flux linkage deviation of the inverter-side inductor when $m=0.85$ and $M=5$. The highest deviation occurs when the reference voltage is halfway between two voltage levels.

value less than $5 \%$ per unit. The limitation is imposed because a high capacitance results in a higher reactive power and a lower power factor. This is normally the case because the AFE controller controls the inverter current instead of the grid current. However, the reactive power of the grid can be controlled to zero by adjusting the inverter reactive power (Hiti et al., 1994).

In the simulations of the MDC, the apparent switching frequency of the grid inverter is in the range of several kilohertz, and thus, quite a low capacitance in the grid filter is needed. In addition, the AFE controller design is relaxed, when the recommendation (capacitance less than $5 \%$ per unit) is adhered to.

The inductance and capacitance of the LCL and the grid determine a resonance frequency for the circuit. The resonance frequency should be in the range of $10 f_{\mathrm{g}}-0.5 f_{\mathrm{sw} \text {,app }}$ to avoid resonance ringing caused by the low-order harmonics of the grid frequency in the lower end 
and the switching-frequency sidebands in the upper end (Liserre et al., 2005).

The resonance frequency can be calculated by

$$
f_{\text {res }}=\sqrt{\frac{L_{\mathrm{g}}+L_{1}+L_{2}}{4 \pi^{2}\left(L_{\mathrm{g}}+L_{2}\right) L_{1} C_{\mathrm{f}}}}=\sqrt{\frac{L_{\mathrm{tot}}}{4 \pi^{2}\left(L_{\mathrm{g}}+L_{2}\right) L_{1} C_{\mathrm{f}}}} .
$$

In order to meet the resonance frequency criteria, the limit values for $C_{\mathrm{f}}$ can be determined by solving for $C_{\mathrm{f}}$ in (3.18) and substituting the limit values for the resonance frequency

$$
\frac{L_{\mathrm{tot}}}{\left(0.5 \omega_{\mathrm{sw}, \mathrm{app}}\right)^{2}\left(L_{\mathrm{g}}+L_{2}\right) L_{1}} \leq C_{\mathrm{f}} \leq \frac{L_{\mathrm{tot}}}{\left(10 \omega_{\mathrm{g}}\right)^{2}\left(L_{\mathrm{g}}+L_{2}\right) L_{1}} .
$$

The upper limit of the capacitor is also affected by the minimum power factor criterion discussed above:

$$
C_{\mathrm{f}, \max }=\min \left\{0.05 C_{\mathrm{b}}, \quad \frac{L_{\mathrm{tot}}}{\left(10 \omega_{\mathrm{g}}\right)^{2}\left(L_{\mathrm{g}}+L_{2}\right) L_{1}}\right\} .
$$

It is obvious from (3.19) that the required capacitance is inversely proportional to the apparent switching frequency. Normally, the maximum value of the capacitor is defined by the power factor criterion. Both inductances of the LCL filter appear in the equations for the resonance frequency and capacitor limit values, but the $L_{2}$ is yet to be determined. Thus, the design process is iterative in nature.

The resistance of the damping resistor $R_{\mathrm{d}}$ should be high enough to provide sufficient damping to avoid oscillation, but its maximum value is determined by the energy dissipation. The efficiency of the converter should not be determined by the damping resistor (Liserre et al., 2005).

The voltage of the filter capacitor is approximately equal to the grid line-to-neutral voltage. Hence, the current of the capacitor can be roughly estimated to give an indication of the losses of the damping resistor. The losses can be approximated by

$$
P_{\text {loss }, R_{\mathrm{d}}}=3\left(U_{\mathrm{LN}} \omega_{\mathrm{g}} C_{\mathrm{f}}\right)^{2} R_{\mathrm{d}}
$$

The effect of $R_{\mathrm{d}}$ is further discussed in Section 3.4.2.

\section{Grid-side inductor}

The value of the grid-side inductance $L_{2}$ can be determined at this point. The peak ripple current of $L_{1}$ was set at $10 \%$ from the nominal phase current amplitude. This ratio can be parameterized as attenuation $A_{\text {inv }}$. This high-frequency content is further suppressed by the sum inductance $L_{\mathrm{g}}+L_{2}$ with an attenuation ratio $A_{\text {grid }}$. Multiplication of these attenuations gives the total attenuation $A_{\text {tot }}=A_{\text {grid }} A_{\text {inv }}$, which describes the ratio of the peak ripple current in the grid to peak nominal current. The attenuation $A_{\text {grid }}$ can be defined by the following expression (Liserre et al., 2005)

$$
A_{\text {grid }}(r)=\frac{1}{\left|1+r\left[1-4 \pi^{2} L_{1} C_{\mathrm{f}} f_{\mathrm{sw}, \mathrm{app}}^{2}\right]\right|} .
$$


In (3.22), $r$ is the ratio of the grid-side total inductance and inverter-side filter inductance. Having established (3.22) and a value for $A_{\text {grid }}$, the inductance ratio $r$ can be solved. The inductance $L_{2}$ is defined using the inductance ratio as

$$
L_{2}=r L_{1}-L_{\mathrm{g}}
$$

At this point, all but two parameters of the LCL filter have been defined. The remaining parameters are the series resistances of the filter inductors. Rockhill et. al. (2011) use a value $0.5 \%$ of the base impedance for their design, and this value is assumed also in the simulation study of this doctoral dissertation:

$$
R_{1}=R_{2}=0.005 Z_{\mathrm{b}}
$$

\subsubsection{Active front end controller}

The previous section presented the design of the grid filter, the objective of which is to filter out the unwanted high-frequency content produced by the inverter switching operation. This section presents the controller that gives the phase voltage instructions to the modulator at the grid interface. The main objectives of the controller are: 1) maintain the DC link voltages of the converter at the reference value, and 2) adjust the reactive power of the converter. The following study is based on the research reported in (Sankala et al., 2014b).

Since each cell in a belt transfers single-phase power, the instantaneous power of a cell has a DC component and a component with two times the frequency that is supplied by the seriesconnected converter (apart from this there are other components related to the switching of the belt transformer and to the motor output frequency, but for simplicity, these are neglected in the analysis). Three cells are connected to a common belt transformer so that each of these cells is part of one phase of a three-phase system. Therefore, the double frequency components are displaced by 120 degrees from each other so that the mean value of the powers of these cells is approximately constant. The three cells connected to the same belt thereby constitute a virtual three-phase system where the instantaneous power is constant compared with the single-phase power having the double frequency component. The energy exchange through the belt transformer provides a natural balancing of the DC link voltages inside one belt. On the other hand, the series connection of the H-bridges is made between different belts, and the modulation method in question (PSCPWM, see Section 3.3) was selected because it enables balanced loading of the series-connected cells.

Thus, there are two mechanisms that naturally balance the DC link voltages in the MDC. 1) The belt transformer balances the DC voltages inside the belt, and 2) the PSCPWM method balances the loading of the cascaded cells. These result in a relaxed requirement for the AFE controller, because it does not need to handle the balance of the DC link voltages. Only the total DC link voltage or the average DC link voltage has to be controlled by the AFE. This means that well-established AFE controller design procedures apply in the MDC, even though the topology is quite a complex one. In the following, an analysis of the controller is given 
and an example design is performed. After that, simulation results are shown demonstrating the controller in action.

Only simulation results are provided for system with active front end an LCL filter, since the grid interface of the prototype system has diode bridges (see Section 3.7).

The AFE system (see Figure 3.9) comprises two controllers: 1) the DC link voltage controller (VC) adjusts the current reference such that the average DC link voltage in the grid-side submodules equals the reference value. 2) The current controller (CC) operates in a rotating coordinate system and adjusts the voltage references in the direct (d) and quadrature (q) axes such that the reference currents are obtained. Both the $\mathrm{VC}$ and $\mathrm{CC}$ employ regular proportional-integral (PI) controllers. When a motor application is considered and the filter is designed as specified in Section 3.4.1, the q-axis current can be controlled to zero to obtain an almost unity power factor in the point of common coupling (PCC).

The voltage equations for the inverter output voltage in synchronous coordinates are:

$$
\begin{aligned}
& u_{\mathrm{d}, \text { inv }}=u_{\mathrm{d}, \text { grid }}-i_{\mathrm{d}}\left(R_{\mathrm{tot}}+\omega_{\mathrm{g}} L_{\mathrm{tot}}\right)+i_{\mathrm{q}} \omega_{\mathrm{g}} L_{\mathrm{tot}} \\
& u_{\mathrm{q}, \text { inv }}=u_{\mathrm{q}, \text { grid }}-i_{\mathrm{q}}\left(R_{\mathrm{tot}}+\omega_{\mathrm{g}} L_{\mathrm{tot}}\right)-i_{\mathrm{d}} \omega_{\mathrm{g}} L_{\mathrm{tot}}
\end{aligned}
$$

The reference inverter voltage vector is calculated by these equations. The first component on the right side of the equations is the measured or estimated grid voltage, the second component is the voltage drop of the filter and grid impedance neglecting the filter capacitor branch, and the third component is the cross-coupling term. The cross coupling is described by the coefficient $k_{\mathrm{cc}}=\omega_{\mathrm{g}} L_{\mathrm{tot}}$ in Figure 3.9.

The transfer function that describes the relation of the current through the inverter-side inductor and the inverter output voltage is defined by (Liserre et al., 2005)

$$
G_{\mathrm{LCL}}(s)=\frac{I_{L_{1}}(s)}{U_{\mathrm{inv}}(s)}=\frac{1}{s L_{1}} \frac{s^{2}+s R_{\mathrm{d}} C_{\mathrm{f}} Z_{\mathrm{LC}}^{2}+Z_{\mathrm{LC}}^{2}}{s^{2}+s R_{\mathrm{d}} C_{\mathrm{f}} \omega_{\mathrm{res}}^{2}+\omega_{\mathrm{res}}^{2}},
$$

where

$$
\begin{aligned}
Z_{\mathrm{LC}}^{2} & =\frac{1}{\left(L_{\mathrm{g}}+L_{2}\right) C_{\mathrm{f}}} \\
\omega_{\mathrm{res}}^{2} & =\frac{\left(L_{\mathrm{g}}+L_{2}+L_{1}\right) Z_{\mathrm{LC}}^{2}}{L_{1}}=\frac{L_{\mathrm{tot}} Z_{\mathrm{LC}}^{2}}{L_{1}} .
\end{aligned}
$$

The output of the PI controllers of the CC are the voltage drops over the filter and the grid sum impedance as described in (3.26) and Figure 3.9. Consequently, the time constant of the current controller can be calculated from the total resistance and inductance

$$
\tau_{\mathrm{LR}}=\frac{L_{\mathrm{tot}}}{R_{\mathrm{tot}}}
$$



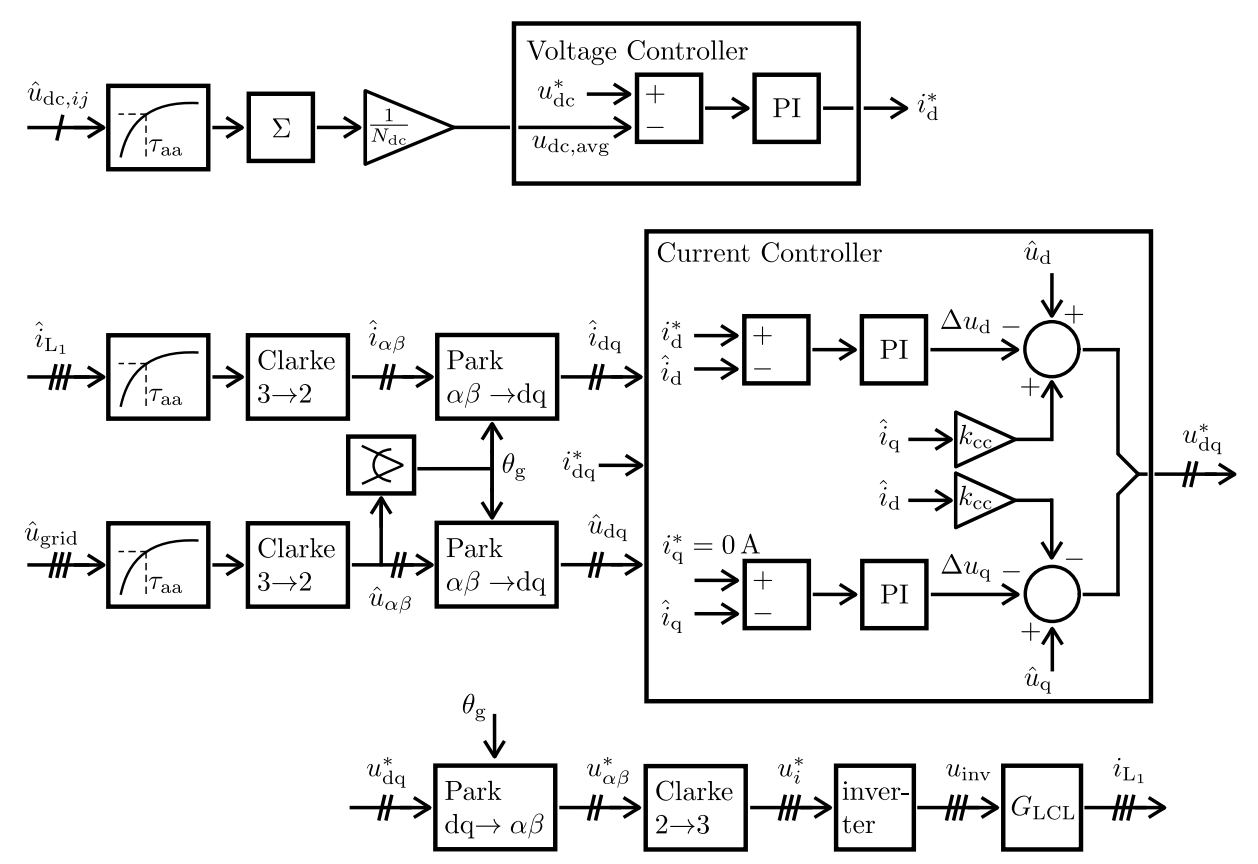

Figure 3.9. Block diagram of an active front end controller. The DC link voltage controller (VC) gives the d-axis current reference for the current controller (CC). The CC operates in a synchronous dq reference frame, and the cross coupling of the axis terms is compensated.

Next, the gain of the CC is tuned with the "technical optimum" criterion using the equation below (Blasko and Kaura, 1997):

$$
K_{\mathrm{P}, \mathrm{CC}}=\frac{\tau_{\mathrm{LR}} R_{\mathrm{tot}}}{3 t_{\mathrm{s}, \mathrm{AFE}}}
$$

Now, the gain and integration time of the PI controllers in the CC are determined, and the integral gain $K_{\mathrm{I}, \mathrm{CC}}$ is defined as the ratio of the proportional gain and the integration time. The closed-loop transfer function of the current-controlled plant can be written as

$$
G_{\mathrm{CC}}(s)=\frac{G_{\mathrm{PI}, \mathrm{CC}} G_{\mathrm{LCL}}}{1+H_{\mathrm{aa}} G_{\mathrm{PI}, \mathrm{CC}} G_{\mathrm{LCL}}},
$$

where $H_{\text {aa }}$ is a first-order low-pass filter defined by the time constant $\tau_{\text {aa }}$ and located in the feedback loop of the system. $G_{\mathrm{PI}, \mathrm{CC}}$ is the transfer function of the PI controller.

The design of the VC begins by determining the dominant pole of the DC link voltage plant. The DC link capacitance of one submodule and an equivalent load resistance produce a time constant:

$$
\tau_{\mathrm{RC}}=R_{\mathrm{eq}} M C_{\mathrm{dc}}=\frac{3 M u_{\mathrm{dc}, \mathrm{cell}}^{2}}{P_{\mathrm{n}}} M C_{\mathrm{dc}},
$$

where $P_{\mathrm{n}}$ is the nominal power of the load and $M$ the number of belts. The time constant of (3.33) can be substituted into the VC PI controller integration time. The DC voltage plant is 
approximated by the following transfer function:

$$
G_{\mathrm{DC}, \mathrm{eq}}=\frac{1}{s M C_{\mathrm{dc}}} .
$$

The closed-loop voltage controlled plant can now be described as follows:

$$
G_{\mathrm{VC}}(s)=\frac{G_{\mathrm{PI}, \mathrm{VC}} G_{\mathrm{CC}} G_{\mathrm{DC}, \mathrm{eq}}}{1+H_{\mathrm{aa}} G_{\mathrm{PI}, \mathrm{VC}} G_{\mathrm{CC}} G_{\mathrm{DC}, \mathrm{eq}}}
$$

where $G_{\mathrm{PI}, \mathrm{VC}}$ is the VC PI controller transfer function and $G_{\mathrm{DC} \text {,eq }}$ is the equivalent transfer function of the DC link. The proportional gain of the voltage controller should be tuned such that a good trade-off between the overshoot and the rise time of the step response is obtained.

\subsubsection{Example LCL filter and AFE controller design}

An example design for the grid interface of MDC is given in this section. The starting point for the design is a motor drive system with the following parameters: $U_{\mathrm{LL}}=4.16 \mathrm{kV}, f_{\mathrm{g}}=$ $50 \mathrm{~Hz}, M=4, P_{\mathrm{n}}=3 \mathrm{MW}, u_{\mathrm{dc}, \text { cell }}=1050 \mathrm{~V}, f_{\mathrm{sw}, \text { app }}=6 \mathrm{kHz}$, and the maximum allowed grid ripple current is $0.02 \sqrt{2} I_{\mathrm{n}} \rightarrow A_{\text {tot }}=0.02$. The example design is for a motor application, and therefore, a unity power factor is assumed at the grid interface.

The base quantities in this system are:

$$
\begin{aligned}
& Z_{\mathrm{b}}=\frac{(4160 \mathrm{~V})^{2}}{3 \times 10^{6} \mathrm{~W}}=5.77 \quad[\Omega], \\
& L_{\mathrm{b}}=\frac{5.77 \Omega}{2 \pi \times 50 \mathrm{~Hz}}=18.4 \quad[\mathrm{mH}], \\
& C_{\mathrm{b}}=\frac{1}{2 \pi \times 50 \mathrm{~Hz} \times 5.77 \Omega}=0.55 \quad[\mathrm{mF}] .
\end{aligned}
$$

The design procedure starts by defining the grid impedance. Assuming that the short-circuit to the nominal current ratio of the possible installations is in the range of $I_{\text {ratio }}=50-1000$ and the grid resistance is $20 \%$ of the total grid impedance $(x=0.20)$, the grid inductance will be from 360 to $18 \mu \mathrm{H}$ according to (3.11).

The LCL filter must be designed such that the total ripple attenuation $A_{\text {tot }}$ is obtained over the whole operating range. Thus, the upper limit of the short-circuit current is used in the design process: $I_{\text {ratio }}=1000 \rightarrow L_{\mathrm{g}}=18 \mu \mathrm{H}, R_{\mathrm{g}}=1.2 \mathrm{~m} \Omega$.

The maximum ripple current in the inverter-side inductor is $10 \%$ of the nominal current amplitude. Thus, the inductance can be determined using (3.17): $L_{1}=0.371 \mathrm{mH}=0.02 \mathrm{pu}$.

Next, an initial guess for the filter capacitor is made: $C_{\mathrm{f}}=0.047 C_{\mathrm{b}}=0.026 \mathrm{mF}$. In order to calculate a value for the grid-side inductor, $r$ is first solved graphically from (3.22). The result is shown in Figure 3.10. Now, the inductance becomes $L_{2}=0.157 \mathrm{mH}=0.009 \mathrm{pu}$. 
According to (3.19) and (3.20), the capacitance should be within the interval $0.024-0.028 \mathrm{mF}$ $(0.043-0.05 \mathrm{pu})$. The minimum limit selection would give a resonance frequency of $3 \mathrm{kHz}$ (the higher limit) if the value of the grid-side inductance is not refined. Recalculation of $r$ with the lower value of $C_{\mathrm{f}}$ would result in a higher value for $L_{2}$. For the sake of the example, the initial guess for $C_{\mathrm{f}}$ and the resulting $L_{2}$ are deemed sufficient and no further iteration is pursued.

The filter was designed for a low-impedance grid to meet the specified ripple attenuation. Contrary to that, the AFE is designed for a high-impedance grid in order to tune the current controller for the longest time constant. The impedance of the grid is calculated by (3.11) using a short-circuit to load current ratio of $50: L_{\mathrm{g}}=0.360 \mathrm{mH}$ and $R_{\mathrm{g}}=23.1 \mathrm{~m} \Omega$.

First, the proportional and integral gains of the $\mathrm{CC}$ are defined. The integration time is calculated by (3.30): $\tau_{\mathrm{LR}}=11 \mathrm{~ms}$. Using (3.31), the proportional gain is obtained: $K_{\mathrm{P}, \mathrm{CC}}=$ 1.776.

Now, all values, except for the damping resistor $R_{\mathrm{d}}$, have been defined. The effect of the damping resistor on the transfer function of the LCL filter and the closed-loop CC plant is shown in Figure 3.11.

As expected, the value of $R_{\mathrm{d}}$ has no effect on the resonance frequency. The peak is smoothed with higher values of the resistor. The value should be high enough to obtain attenuation at the resonance frequency on the closed-loop transfer function. In this example design, $0.5 \Omega$ is sufficient for that but a value $0.7 \Omega$ is chosen. The losses of the damping resistor are then estimated by (3.21): $P_{\text {loss }, R_{\mathrm{d}}} \approx 800 \mathrm{~W}$. This corresponds to $0.027 \%$ of the nominal power of the converter, and is thus low enough.

With the chosen $R_{\mathrm{d}}$, the effect of the grid impedance variation on the transfer functions of the LCL and the closed-loop CC plant is shown in Figure 3.12. With a lower grid impedance, the attenuation of the resonance peak increases and the frequency of the peak is pushed slightly higher.

Next, the design of the voltage controller is discussed. The input signal, $u_{\mathrm{dc}, \text { avg }}$, is the average

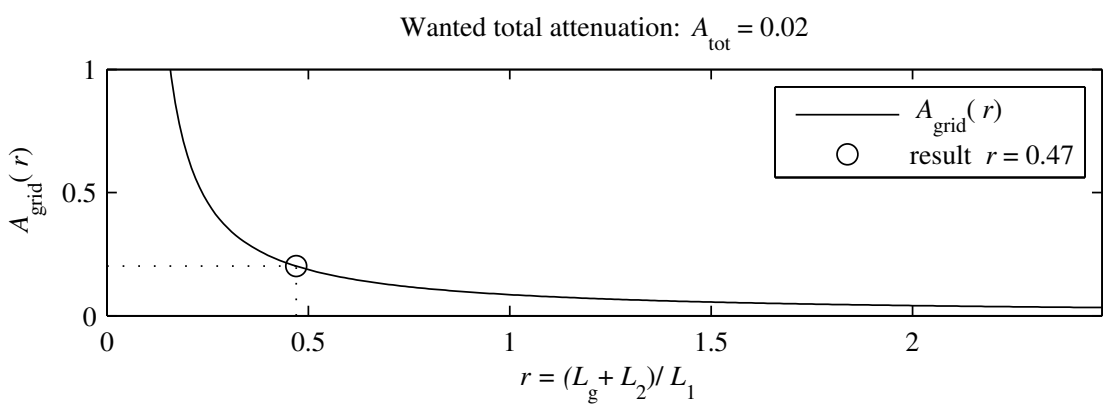

Figure 3.10. Graphical solution of the inductance ratio $r$ in the example LCL design. 


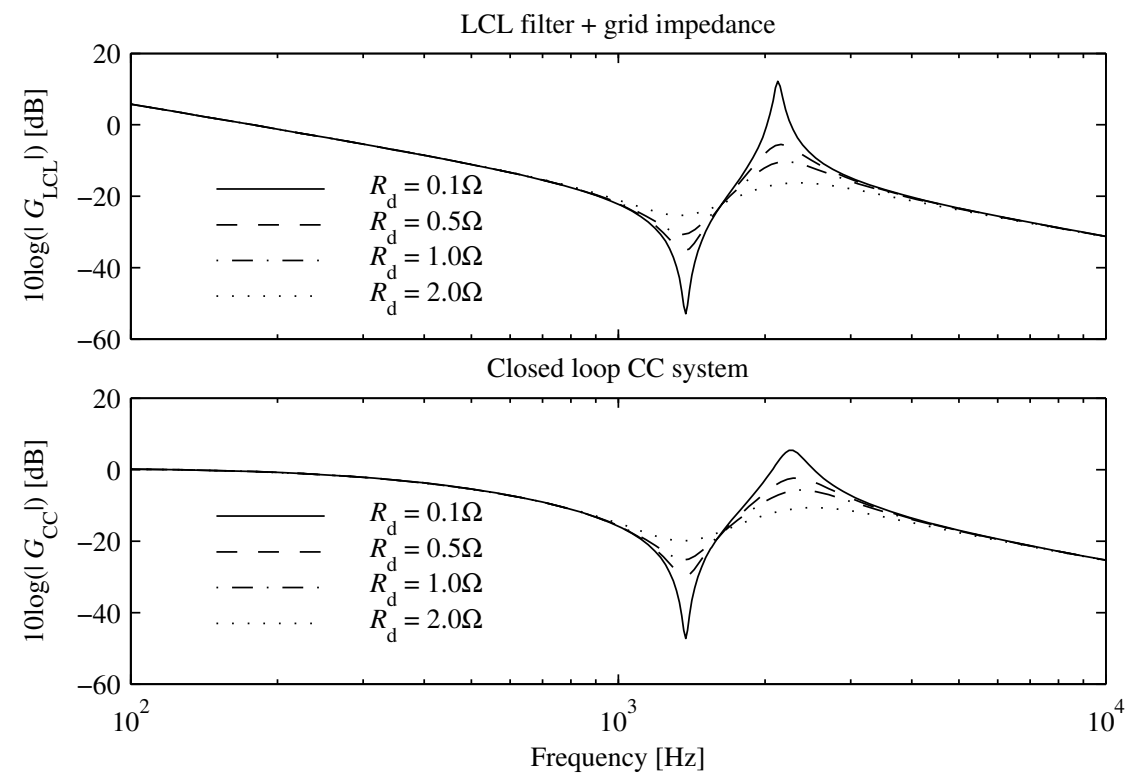

Figure 3.11. Effect of the damping resistor value on the transfer functions of the LCL filter and the closed-loop current-controlled plant.
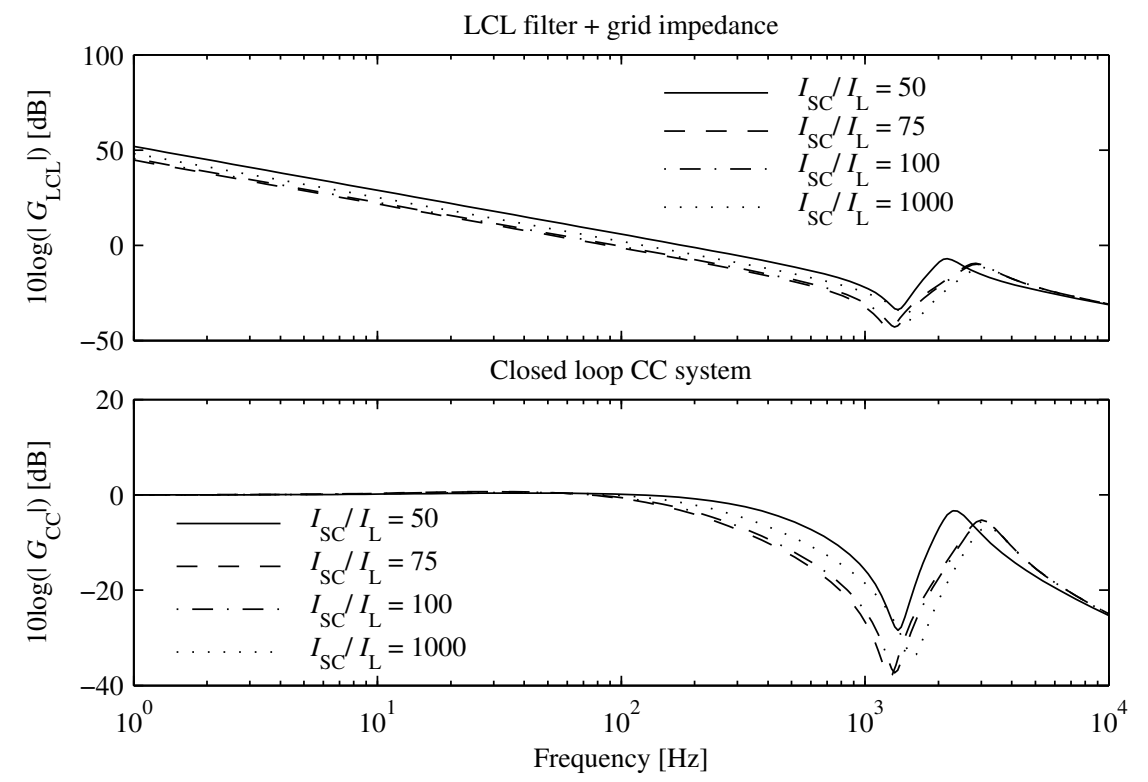

Figure 3.12. Effect of the grid impedance on the transfer functions of the LCL filter and the closed-loop current-controlled plant. 
value of all DC links on the grid side of the converter. The integration time of the controller is given by (3.33): $\tau_{\mathrm{RC}}=17.6 \mathrm{~ms}$. The proportional gain is tuned manually to obtain a smooth step response of the closed-loop VC plant, the rise time of which is longer than the rise time of the closed-loop CC plant. The obtained proportional gain is $K_{\mathrm{P}, \mathrm{VC}}=1.70$.

Figure 3.13 shows the step responses of the closed-loop CC and VC plants (see (3.32) and (3.35)) with four different grid impedances. The solid line represents the high grid impedance that was used in the design of the AFE. In the $\mathrm{CC}$, the lower grid impedance results in a longer time constant and a higher overshoot, but the variation is not significant. With the VC, the decrease in the grid impedance increases the overshoot.

Finally, a simulation result is shown where the step responses of the VC and CC controllers are tested with a high-impedance grid. The system is initially in the nominal operating point and in a steady state. The response of the average DC link voltage is quite close to that of Figure 3.13. The response of the current controller is also close to the expected curve.

Figure 3.15 shows the steady-state performance of the MDC with four belts in the nominal operating point (output active power $3 \mathrm{MW}$ ). The grid-side current waveform is free of switching frequency content, but there are clearly some harmonics on top of the fundamental wave. The inverter-side currents of the filter have the same harmonic content plus the switching frequency content. The amplitude of the stepped phase voltage is lower on the motor side than on the grid side as a result of a voltage drop that occurs over the transformer.

It is clear from these simulation results that there is room for improvement in the system performance. However, considering the scope of the doctoral dissertation, further development is omitted.

The design principles presented in this section are used in all simulations throughout the dissertation. The LCL filter and the AFE controller are individually designed for each number of belts. The parameters used in the various simulations are presented in Appendix B.
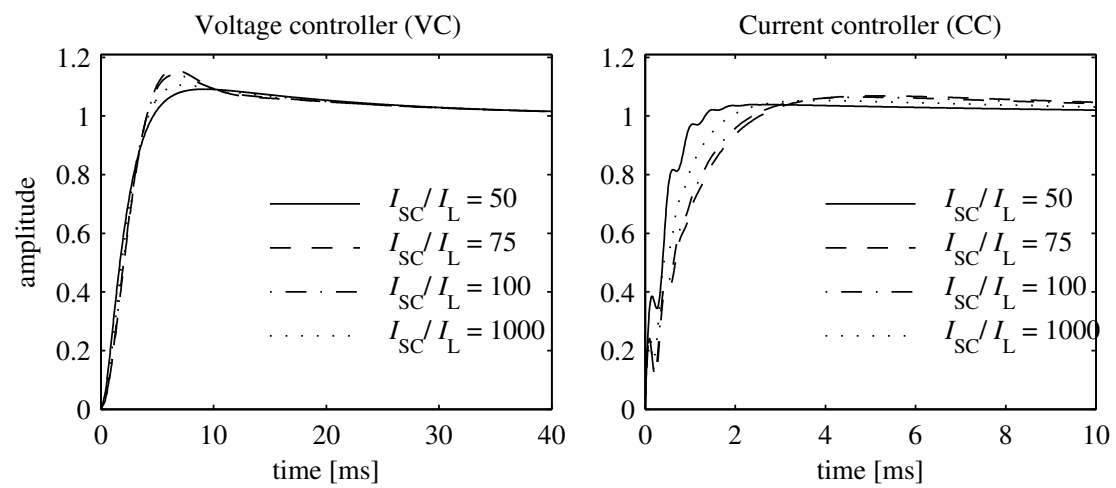

Figure 3.13. Effect of the grid impedance on the step response of the example AFE controller design. 

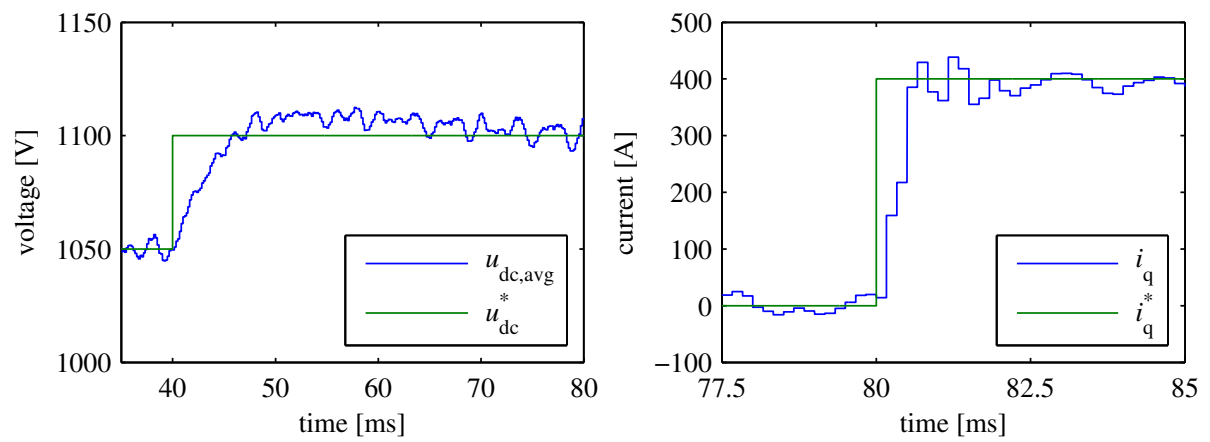

Figure 3.14. Simulated step responses of the VC and CC controllers. The DC link voltage reference step of $50 \mathrm{~V}$ is introduced at $t=40 \mathrm{~ms}$ and the reactive current reference step of $400 \mathrm{~A}$ at $t=80 \mathrm{~ms}$.
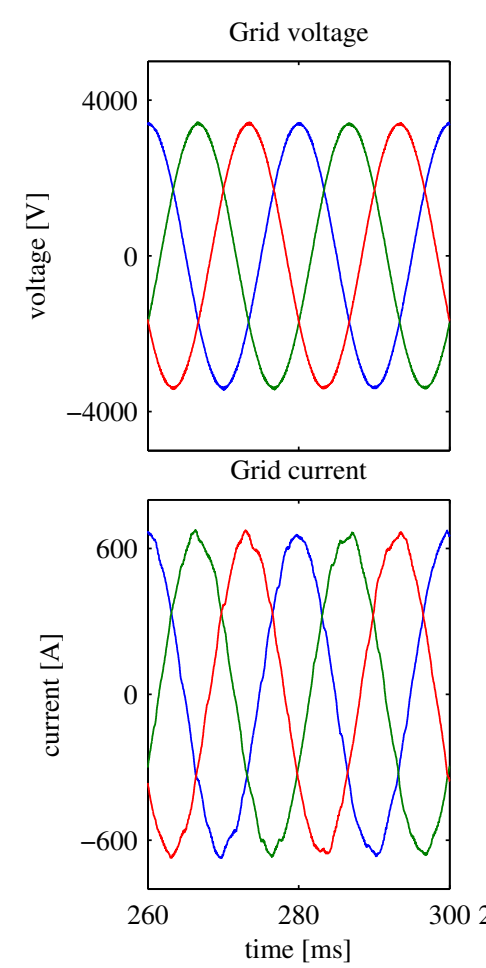

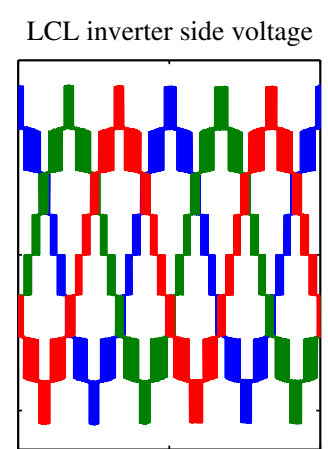

LCL inverter side current

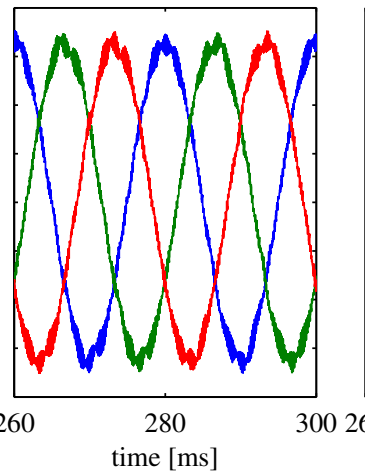

Motor voltage
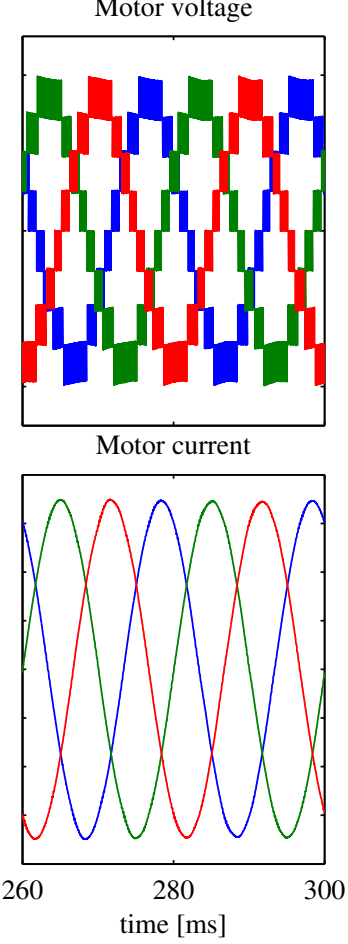

Figure 3.15. Steady-state performance of the MDC with the designed LCL filter depicted with threephase input and output waveforms.

\subsubsection{Assisted DC link voltage control}

While the PSCPWM method provides the cells of the CHB inverter with balanced loading, there may be situations where the DC link voltages are not balanced. For example, unequal 
winding impedances in the medium-frequency transformer link will degrade the balancing effect of the DC link voltages inside one belt. Another instance is a multiport application: for example, two belts are connected in series on the grid side and both belts have independent motor loads. In this case, the DC link voltages will be unbalanced if the powers of the two motors are not equal. DC link voltage balancing has been reported problematic with a multiport application of the UNIFLEX-PM converter (UNIFLEX-PM, 2007).

In the UNIFLEX-PM system (universal and flexible power management), the converter structure is very close to the structure of the MDC; see Section 2.2.3. The balance of the DC link voltage of the series-connected cells in the UNIFLEX-PM converter is handled by individually controlling the modulation index of each cascaded cell (Watson et al., 2007). The control method is described in detail in one of the UNIFLEX-PM project EU deliverables (UNIFLEX-PM, 2007). Since this algorithm seems to yield good results with the UNIFLEX converter, the algorithm was tested with the MDC also. The control method is depicted in Figure 3.16.

The basic idea of the controller is that the lower the DC link voltage of a cell is, the higher its modulation index should be. However, the sum voltage produced by the series-connected cells has to match the phase voltage reference value, $u_{\mathrm{i}}^{*}$, commanded by the AFE controller and scaled by the total DC link voltage compensator. This is achieved by applying a PI controller to each cell. The input for the PI controller is the DC link voltage error filtered by a low-pass filter and scaled by the inverse of the reference DC link voltage value:

$$
u_{\mathrm{err}, i j}=\frac{u_{\mathrm{dc}}^{*}-u_{\mathrm{dc}, \mathrm{ij}}^{*}}{u_{\mathrm{dc}}^{*}} .
$$

The average of the outputs of all PI controllers for one inverter output phase is calculated by

$$
\Delta_{\mathrm{avg}, i}=\frac{1}{M} \sum_{j=1}^{M} \Delta_{i j},
$$

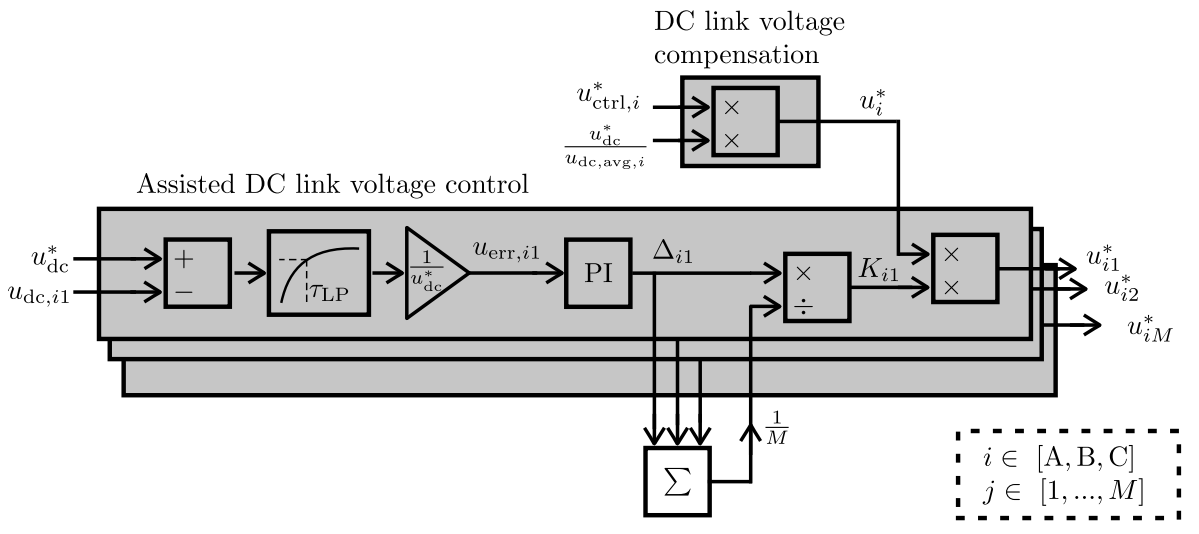

Figure 3.16. Block diagram of the assisted DC link voltage control. Individual cell voltage references are adjusted with PI controllers while the sum of the cell voltages is kept equal to the voltage commanded by the controller. 
where $\Delta_{i j}$ is the output of one PI controller. The individual PI controller outputs are then divided by the average value. This calculation gives each cell a coefficient, $K_{i j}$, which is used to scale the phase voltage reference value to get individual cell references

$$
u_{i j}^{*}=K_{i j} u_{i}^{*}, \quad \text { where } \sum_{j=1}^{M} K_{i j}=M .
$$

This method ensures that the sum of the cell voltages equals the total reference phase voltage. The PI controllers have to be initialized to value 1 (with $u_{\mathrm{err}, \mathrm{ij}}=0 \mathrm{pu} \rightarrow u_{\mathrm{ij}}^{*}=u_{\mathrm{i}}^{*}$ ).

The effectiveness of the DC link voltage control method is assessed by a simulation; see Figure 3.17. The initial conditions match those of Figure 3.5. With the assisted DC link voltage control turned on, the $-200 \mathrm{~V}$ error in belt $1 \mathrm{DC}$ links is corrected within $30 \mathrm{~ms}$. Thus, the time needed to balance the DC link voltages is reduced by $85 \%$ by the assisted DC link voltage control.

The properties of the assisted DC link voltage control under the load power sign change condition are assessed by another simulation. The power flow of the motor is reversed during a $100 \mathrm{~ms}$ time period by ramping the applied external torque from the nominal value to the negative nominal value while the motor controller keeps the speed of the motor constant.

The set of subplots on the left in Figure 3.18 demonstrate the behavior when the assisted DC link voltage controller is turned off. Smooth operation is obtained while the DC link voltages overshoot slightly from the reference value. With the controller turned on (sublots on the right), a catastrophic failure occurs when the DC link voltages return close to the reference value. During the transient, all the DC link voltages are above the reference value. The outputs of the PI controllers reach the minimum saturation limit very quickly while none of the DC links follows along. When the transient state is close to its end, the DC link voltage

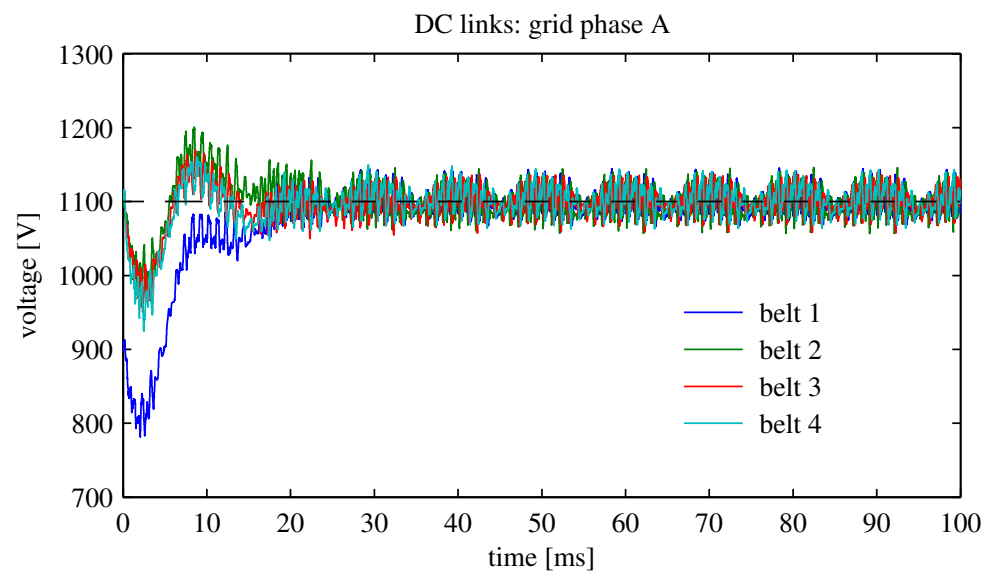

Figure 3.17. Demonstration of the effectiveness of the assisted DC link voltage control when $M=4$. The reference DC link voltage of $1100 \mathrm{~V}$ is shown by a black dashed line. 
in cell 4 goes below the reference value. From this point onwards, the controller coefficients $K_{i j}$ diverge from unity. The saturated state of the integrators inside the PI controllers drives the system unstable.

The problem described in Figure 3.18 is triggered whenever the sign of the DC link voltage error in all the DC links is the same. A solution to this problem is left for future research.

The structure of the assisted DC link voltage controller enables an interesting feature: each DC link can have an independent reference value of its own. However, there are some limitations: First, because of the natural balancing of the DC links inside one belt, the DC links of the belt need equal references. Second, the average of the DC link voltage references must match the reference voltage of the main DC link controller. Otherwise, the two controllers
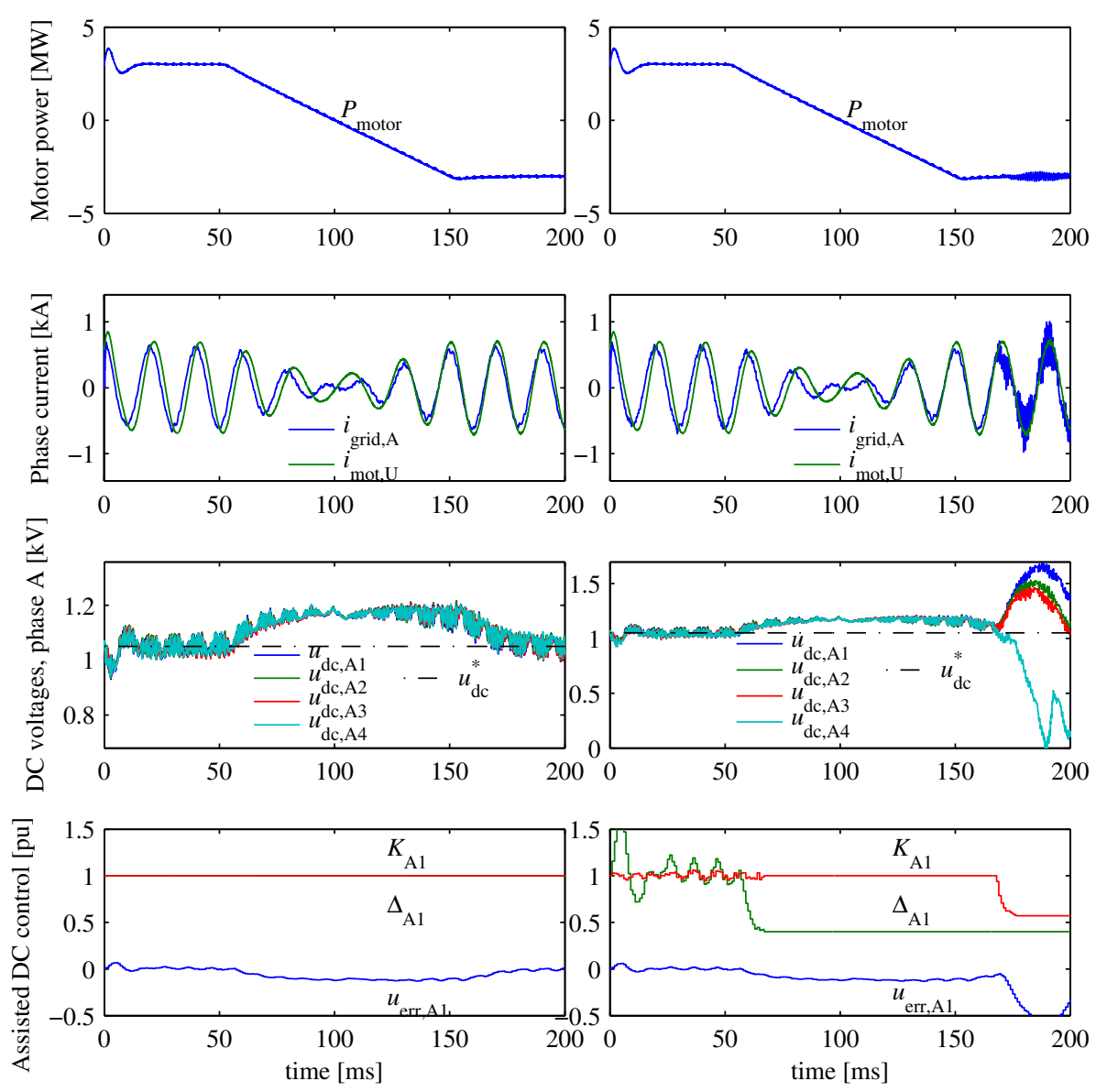

Figure 3.18. Simulation results in the case of a power sign change; the assisted DC link voltage control turned OFF on the left and ON on the right. 
have setpoints with conflicting effects on the controlled variable. However, no clear application for an intentional DC link imbalance is known, and thus, further analysis of this property is omitted.

\subsubsection{Grid filter configurations}

This section considers different approaches to provide the LCL filter at the grid interface. The traditional LCL filter is a nonmodular component, which in the case of a highly modular topology such as an MDC can be regarded as a defect. In the following, two approaches are investigated that aim to improve the modularity of the filter.

An increase in modularity can be obtained if the components of the filter are distributed among the cascaded cells. In other words, the filter itself is cascaded. As to the structure of the LCL filter, the first obvious approach would be to distribute the inverter-side inductance into multiple individual inductors, one for each cell. The capacitor and the grid-side inductor would still be nonmodular.

The second approach is to divide the inverter-side inductor and the filter capacitor (Huang et al., 2008). In this approach, each H-bridge cell has an individual LC filter, and the gridside inductor is the only nonmodular component. The investigated filter structures are shown in Figure 3.19.

\section{Distributed inverter-side inductance}

The filter topology with a distributed inverter-side inductance is shown in Figure 3.19a. The cascaded cells and inductors are connected in series, and therefore, the filter output equals the centralized LCL filter case if the individual inductance value is selected as $L_{1 \text {,dis }}=M^{-1} L_{1}$.

Thus, the total inductance matches the case of the centralized LCL filter, and therefore, this method requires no changes to the total inductance calculation or the AFE controller design. The total inductance is calculated by (3.17). The series resistance of the filter is assumed to decrease in the same proportion as the inductance. Simulated waveforms with the distributed inductance are shown in Figure 3.20. The simulation is performed with four series-connected belts connected to a $4.16 \mathrm{kV}$ grid driving a $3 \mathrm{MW}$ induction motor in the nominal operating point.

The simulation result verifies the functionality of the distributed inductance. However, the increase in modularity is not as straightforward as it appears. As discussed in Section 3.4.1, the amount of the total inverter-side inductance is inversely proportional to the apparent switching frequency and the current ripple, and directly proportional to the submodule DC link voltage. Assuming that the DC link voltage and the current ripple are constant with a different number of cascaded belts, only the switching frequency has an impact on the inductance. 
When the voltage rating of the converter is increased by connecting additional belts in series, the total inductance on the inverter side is increased with the distributed inverter-side inductance filter construction. At the same time, the apparent switching frequency increases if the individual IGBT switching frequency is kept constant. On the other hand, if the apparent switching frequency is kept constant, the inductance should also be kept constant,

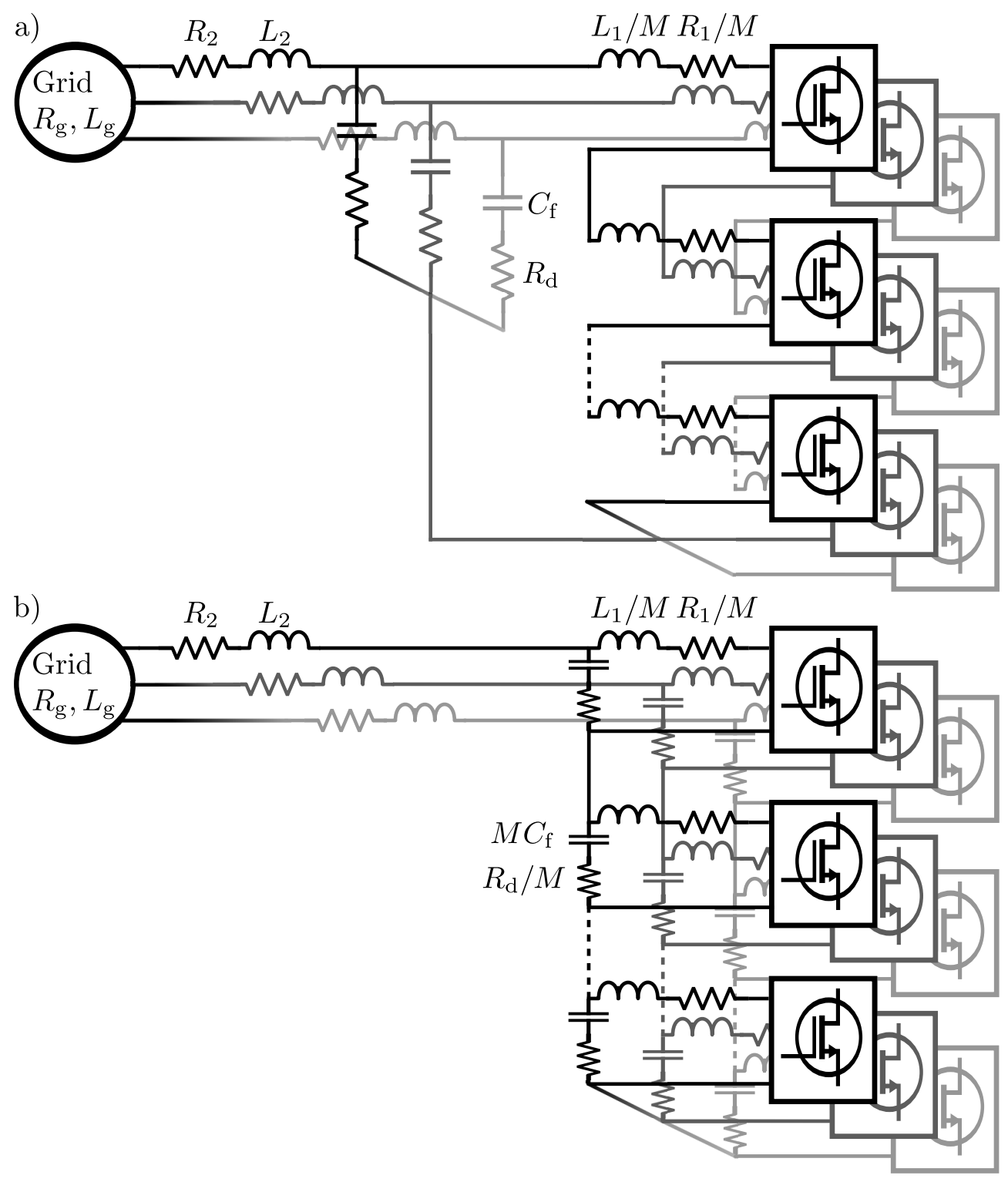

Figure 3.19. Distributed structures of the LCL filter. a) Distributed inverter-side inductance, b) distributed LC filter. 


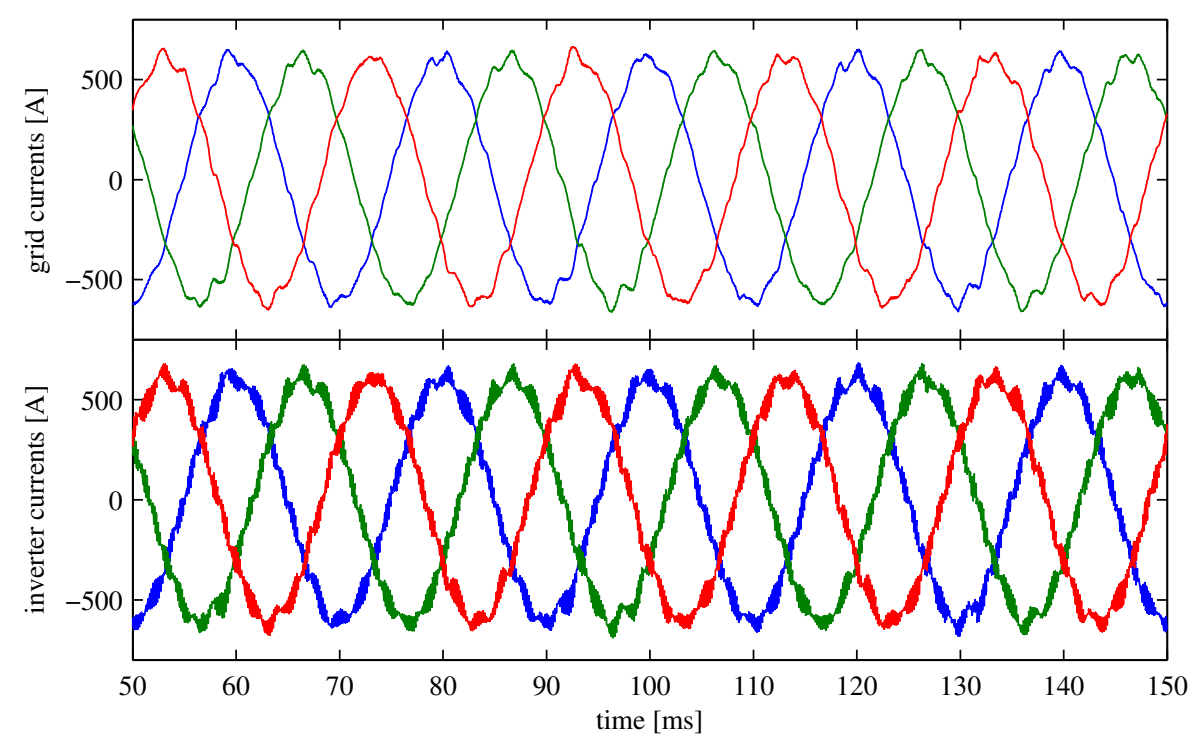

Figure 3.20. Simulated three-phase currents of the grid and the inverter with a distributed inductance on the inverter side of the filter.

but the added belts increase the inductance. This means that even though the inductance is distributed, the target of making the system more modular is not achieved.

\section{Distributed LC filter}

Another option to construct a distributed filter is a distributed LC filter. In this configuration, the grid-side inductance is still one element, but the capacitor and the inverter-side inductance are distributed to the cells; see Figure 3.19b. In the output of each cell there is an LC lowpass filter. This filter reaches the same filtering result as the distributed $\mathrm{L}$ and centralized LCL filters. In order to obtain the same total capacitance, the single capacitors have to be dimensioned for a $M$ times as high capacitance, but at the same time, the voltage rating of a single capacitor reduces in the same ratio (Huang et al., 2008).

However, this filter requires a high inverter-side inductance compared with the other two filter configurations. The reason is that each LC filter experiences a pulsed input waveform at the frequency of a single H-bridge cell, $f_{\mathrm{sw}, \text { cell }}=2 f_{\mathrm{sw}, \mathrm{IGBT}}$. In the other two configurations, the frequency of the input is $M f_{\mathrm{sw} \text {,cell }}$. At these frequencies, the impedance of the filter is dominated by the inductance, not the capacitance or resistances.

According to Huang et al., the inverter-side inductance can be calculated similarly as in the case of a distributed L filter. Even though this method results in the same filtering result in 


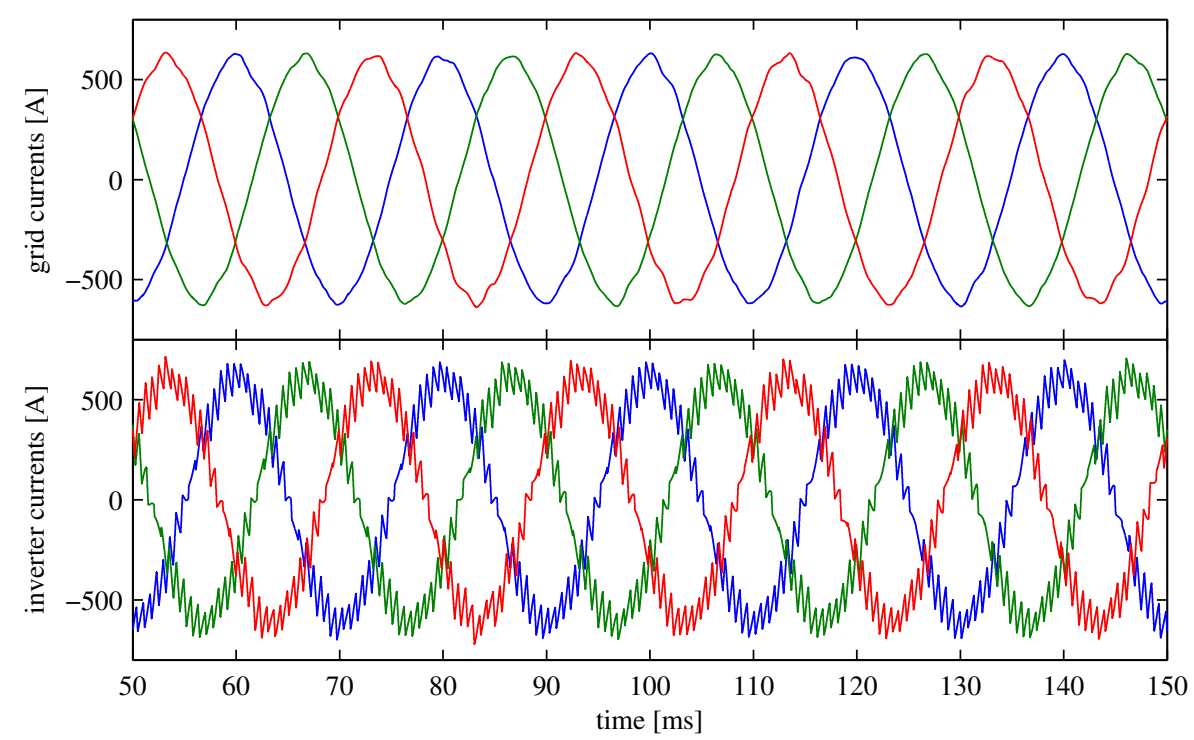

Figure 3.21. Simulated three-phase currents of the grid and the inverter with the distributed capacitance and inductance on the inverter side of the filter. The inverter currents in the bottom figure are the output currents of cells A1, B1, and C1.

theory, the practice is different. The LC filter has a very low impedance at the switching frequency of a cell, and the output current of an H-bridge cell rises to 2-3 kA. This results in a very high voltage ripple at the DC links and cripples the operation of the converter.

Figure 3.21 shows the simulation result when the total inductance on the inverter side is $1000 \%$ of the total inductance in the case of the centralized LCL filter and the total capacitances are equal. As a result of the high total inductance, the low-order harmonics of the grid currents are better damped compared with the simulation result of the previous section. However, the very high inductance is not high enough to sufficiently damp the oscillation at the output current of each cell. Additionally, the high inductance results in a relatively high voltage drop at the fundamental frequency, and the output voltage range of the grid inverter is thus smaller. Considering the modularity of the filter, the distributed LC filter is no different from the distributed $\mathrm{L}$ filter.

\subsection{Load interface}

The MDC is not only dedicated to motor drives. It can also be used in generator applications and in the interconnection of grids of different frequencies and phase angles. While the previous section dealt with the connection to the supplying grid, this section describes the topology-specific issues on the load side. The modulation method used for the CHB 
configuration that drives the load is the PSCPWM (see Section 3.3) similarly as at the grid interface.

CHB inverters have been successfully used in motor drives both in the industry and academia. An MDC as a topology does not pose new challenges to the load interface because the DC link voltage levels are adjusted with the grid-tied CHB as discussed in Section 3.4. Thus, the issues involved in the details of the motor control are omitted from the analysis of this doctoral dissertation, and only a brief explanation of the applied method is given.

This section focuses on MDC-specific issues on the load side with a special reference to the voltage transformation capability of the MDC by combining series and parallel connections of H-bridges or using a delta connection of H-bridges instead of a star connection. Further, a multiport operation of the MDC is discussed. Before going into details of the above-mentioned configurations, the applied motor controller is described in brief.

\subsubsection{Motor control: FOC}

In the simulations and in the case of a motor drive, a closed-loop vector control is used (also known as the field-oriented control, FOC). The FOC is a well-known method and is used especially in high-performance drives. In the FOC, the three-phase quantities are transformed into two phases in a rotating coordinate system. The rotation can be synchronized with any rotating vector, but a common approach is to use an estimated rotor flux linkage vector. With synchronous motor drives, the orientation is obtained with a position sensor, but with asynchronous motors, the slip frequency must be taken into account. With this orientation, the magnetizing current of the motor and the torque-producing current can be directly adjusted to modify the magnetization or torque of the motor. Open-loop control methods (also known as scalar control) such as the traditional $U / f$ curve control could be used. In fact, one embodiment of the scalar control is used in the experimental device.

All simulations are performed using a switching model with a very short sampling time (in the order of a microsecond). The FOC is preferred over the much simpler volts per hertz control in these simulations because the system obtains a much faster transient recovery, and thereby, the steady state is reached much faster thus reducing the overall simulation time. In practice, the scalar control may be the preferred choice especially in low-performance applications such as fans and pumps.

The simulations are carried out using Simulink, and specifically, the SimPowerSystems toolbox. This toolbox offers an induction machine model that outputs the rotor flux linkage vector. Thus, a direct feedback for controller orientation is obtained from the motor model, and no effort is put into the estimation of the correct orientation. This simplifies the model and helps in focusing on the matter at hand: the topology-specific issues on the load side of the converter.

Figure 3.22 shows a block diagram of the motor controller used in the simulations. The input and output values of the controller are real-world values (e.g. amperes, voltages), but the 


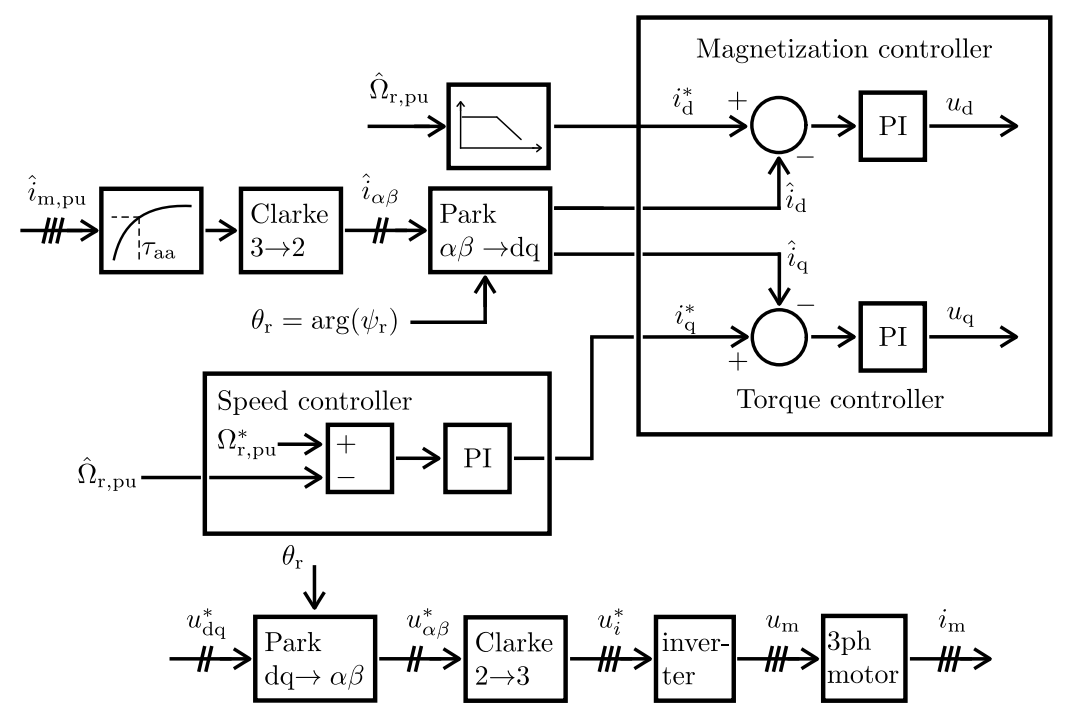

Figure 3.22. Block diagram of the field-oriented controller. Three PI controllers are used. The speed controller outputs a q-axis current reference. The d-axis reference is obtained from a lookup table according to the speed of the motor. The output of the magnetization and torque controllers are the dand q-axis voltage components.

controller operates on the per unit scale. After the input filtering and sampling, the input quantities are transformed into the per unit scale by

$$
\begin{aligned}
i_{\mathrm{m}, \mathrm{pu}} & =\frac{i_{\mathrm{m}}}{\sqrt{2} I_{\mathrm{m}, \mathrm{nom}}}, \\
\Omega_{\mathrm{r}, \mathrm{pu}} & =\frac{p_{\mathrm{m}} \Omega_{\mathrm{r}}}{2 \pi f_{\mathrm{m}, \mathrm{nom}}}
\end{aligned}
$$

where $\Omega_{\mathrm{r}}$ is the mechanical rotation speed of the rotor in radians per second, $p_{\mathrm{m}}$ the number of pole pairs in the motor, and $I_{\mathrm{m} \text {,nom }}$ and $f_{\mathrm{m} \text {,nom }}$ are the motor nominal phase current and frequency. The controller output is the voltage instruction to the modulator, and it is transformed from the per unit scale into volts by

$$
u_{\mathrm{m}}=u_{\mathrm{m}, \mathrm{pu}} \sqrt{\frac{2}{3}} U_{\mathrm{m}, \mathrm{nom}},
$$

where $U_{\mathrm{m} \text {,nom }}$ is the nominal line-to-line voltage of the motor. Note that also the modulator operates on the pu scale, but there 1 pu corresponds to the nominal DC link voltage level, and therefore, the voltage $u_{\mathrm{m}}$ has to be scaled with $1 / u_{\mathrm{dc}}^{*}$.

The motor controller parameters are tuned manually. The torque and flux controllers use the same parameters because the motor is symmetric in the direct and quadrature axes. For example, the parameters used in the simulations with four belts supplying a $4.16 \mathrm{kV}, 3 \mathrm{MW}$ motor are $K_{\mathrm{Pd}}=K_{\mathrm{Pq}}=0.5, T_{\mathrm{Id}}=T_{\mathrm{Iq}}=10 \mathrm{~ms}$, and $K_{\mathrm{Ps}}=50, T_{\mathrm{Is}}=20 \mathrm{~ms}$. The controller sample time equals the period of the apparent switching frequency on the motor side $(6 \mathrm{kHz})$. 
The induction motor used in the simulations is characterized by the parameters listed in Table 3.4 .

Table 3.4. Parameters of the induction machine model used in the simulations. The same per unit values are used regardless of the motor power rating. The absolute values are given for a $3 \mathrm{MW}, 4.16 \mathrm{kV}$ motor as an example.

\begin{tabular}{llll}
\hline Element & Symbol & Value [pu] & $3 \mathrm{MW}$ motor \\
\hline Nominal frequency & $f_{\mathrm{m}, \mathrm{nom}}$ & 1.00 & $50 \mathrm{~Hz}$ \\
Nominal voltage, line-to-line & $U_{\mathrm{m}, \mathrm{nom}}$ & 1.22 & $4160 \mathrm{~V}$ \\
Motor pole pairs & $p_{\mathrm{m}}$ & 2 & 2 \\
Stator resistance & $R_{\mathrm{S}}$ & 0.01 & $48.0 \mathrm{~m} \Omega$ \\
Stator leakage inductance & $L_{\sigma \mathrm{s}}$ & 0.10 & $1.50 \mathrm{mH}$ \\
Magnetization inductance & $L_{\mathrm{m}}$ & 3.00 & $45.9 \mathrm{mH}$ \\
Rotor resistance & $R_{\mathrm{r}}$ & 0.01 & $48.0 \mathrm{~m} \Omega$ \\
Rotor leakage inductance & $L_{\sigma \mathrm{r}}$ & 0.10 & $1.50 \mathrm{mH}$ \\
Moment of inertia & $\mathrm{J}$ & & $20 \mathrm{kgm}^{2}$ \\
\hline
\end{tabular}

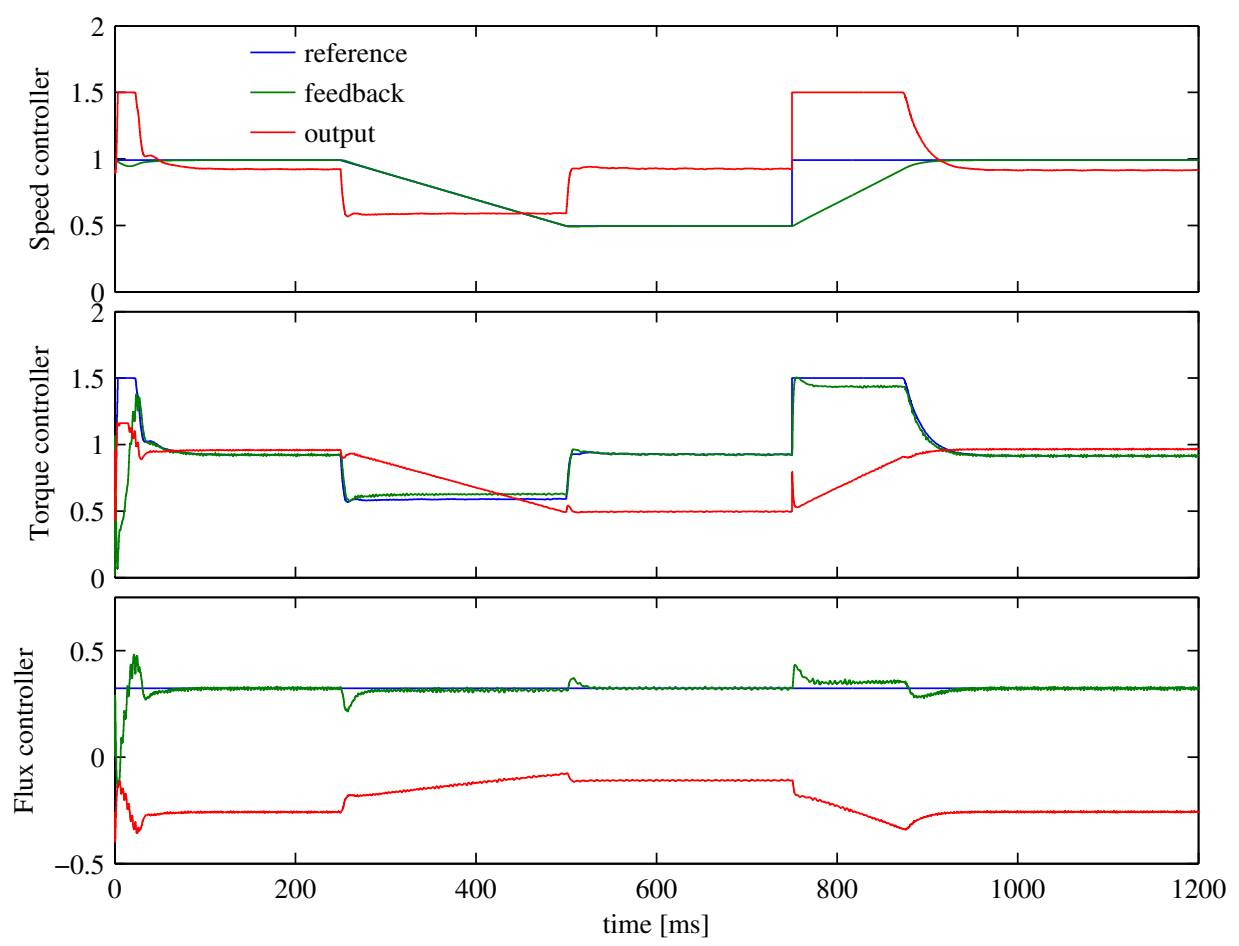

Figure 3.23. Field-oriented controller operation. The deceleration of the motor produces a transient in all controllers when braking of the motor starts and ends. The acceleration of the motor produces the third transient. 
The operation of the controller is tested by simulations; see Figure 3.23. In the simulation, the motor is initially operated under nominal torque and speed. The speed is then reduced from the nominal value to half speed with a $250 \mathrm{~ms}$ ramp. After the transient produced by the braking of the motor, the speed reference is stepwise increased to $1.0 \mathrm{pu}$ to accelerate the motor. As a result, the torque current reference jumps to $1.5 \mathrm{pu}$, which is the saturation limit set for the speed controller.

The performance of the motor controller is smooth in the braking operation. In the acceleration, the speed reference is increased stepwise, and twice the nominal torque is used in the acceleration. The power of the motor increases with the speed, and consequently, the DC link voltage ripple and voltage drop over the transformer increase. Because of the behavior of the DC voltages, perfect tracking of the set point is not obtained during the acceleration. Increasing the speed reference with a ramp in the acceleration or limiting the output of the speed controller are viable solutions to eliminate the problem. No further optimization of the controller is pursued because the topology as a whole is under review, and the presented controller meets the required functionality in the scope of this doctoral dissertation.

\subsubsection{Parallel connection of $\mathbf{H}$-bridges}

A CHB inverter is a flexible system. Isolated cells can be configured in many different ways to produce various output combinations. This enables any $\mathrm{CHB}$ inverter (including the MDC) to be used for voltage transformation. The first of the three possible voltage transformation methods is discussed here: parallel connection of H-bridges. Figure 3.24 depicts a combined series-parallel connection, which produces a voltage transformation ratio of 0.5 .

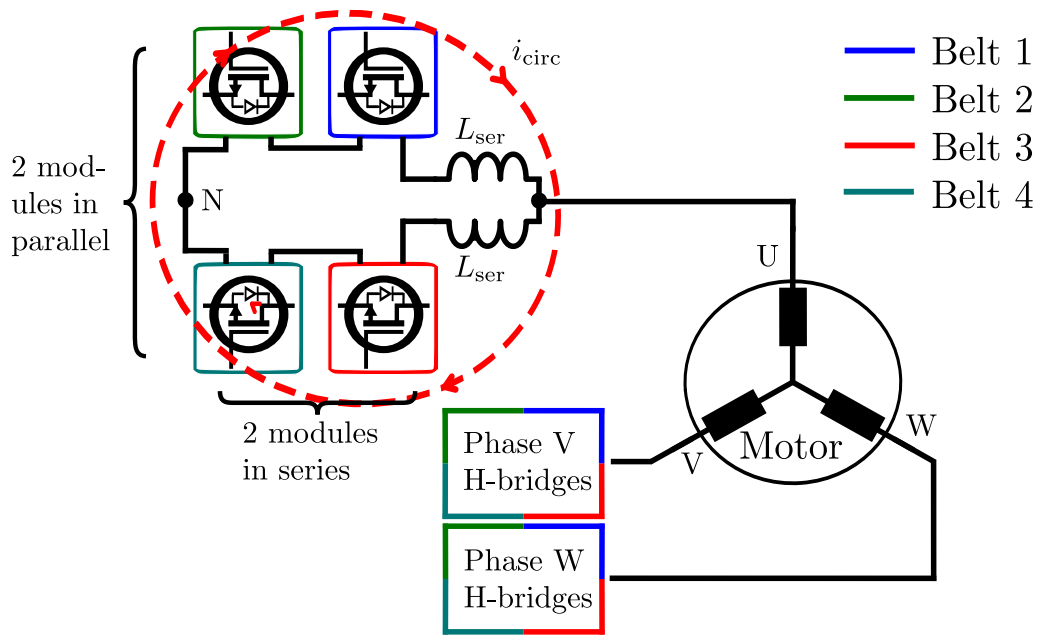

Figure 3.24. H-bridge cells of four belts are connected in a combined series-parallel configuration to drive the motor load. The different belts are indicated by colored cell borders. The parallel-connected branches are coupled through a filter inductor. 
In the MDC, the number of belts $M$ equals the number of $\mathrm{H}$-bridge cells per phase. The amplitude and number of voltage steps of the phase voltage are determined by the number of cascaded cells, $M_{\mathrm{c}}$. The current rating is defined by the number of parallel cells, $M_{\mathrm{p}}$. The total number of belts is $M=M_{\mathrm{c}}+M_{\mathrm{p}}$.

For example, a $3.3 \mathrm{kV}$ grid voltage may be transformed into $1.1 \mathrm{kV}$ by employing three belts, $M=3$. All belts are cascaded on the grid side, but on the load side the three belts are parallel connected. $3.3 \mathrm{kV}$ cannot be transformed for instance into $2.3 \mathrm{kV}$ because that would require two cascaded belts and two parallel belts $\left(M_{\mathrm{c}}+M_{\mathrm{p}}=4>3\right)$. It is noteworthy that the combined series-parallel connection is not feasible with any odd number of belts.

A simulation result is shown in Figures 3.25 and 3.26 where a series-parallel connection is employed to transform $4.16 \mathrm{kV}$ grid voltage into a $2.3 \mathrm{kV}$ supply voltage of a $3 \mathrm{MW}$ induction motor. On the motor side, belts 1 and 2 are connected in series, and correspondingly, belts 3 and 4 are in series. These two series-connected sets are then connected in parallel. In the first figure, one of the input and output phases is shown while the second figure shows the motor power and rotation speed. A few observations can be made:

1. The motor voltage and frequency vary as the speed of the motor changes.

2. At the start and end, the speed is at the nominal value, and the voltage of the motor is approximately half of the grid voltage.

3. At the nominal speed, the motor current is double compared with the grid current.

4. The performance of the parallel-connected system is smooth.

The parallel connection of inverters is known to produce circulating currents (Itkonen, 2010). This behavior is not present in the input and output waveforms or mechanical behavior of the motor in Figure 3.25. There are two mechanisms causing this problem. First, the DC link voltages of the parallel units deviate from each other giving rise to circulating currents. Second, the parallel units are driven with the same modulation signals such that their intended output voltages match. However, the output voltage switching edges include jitter as a result of the unequal switching times of the semiconductors and delays in the gate driver circuits. Nonetheless, no artificial jitter is included in the simulation, and the leakage inductances match.

Taking a look beyond, the input and output ports reveal that there are circulating currents in the simulation even though the winding impedances match and no artificial jitter is included. The current is produced by the fluctuating DC link voltages. The instantaneous value of the DC link voltage of a submodule is affected by the grid-side modulation, the energy transfer through the transformer link, and the motor-side modulation. The following conclusions can be made from the simulation results:

1. The roles of belts 1 and 3 are the same at the motor interface. This means that the output cells are modulated with exactly the same gate signals. In principle, this does not lead to an imbalance between the DC link voltages.

2. At the grid interface, belt 1 and 3 have different modulating signals, and both produce a proportion of their own to the total output voltage of the grid CHB. This leads to a difference 
between the DC link voltage fluctuations in belts 1 and 3. However, the average value of the DC links in these belts match.

3. The previous remarks are applicable to belts 2 and 4 also.

4. Belts 1 and 2 are in series and connected in parallel with the series connection of belts 3 and 4. Consequently, the combined effect of modulation of all belts influences the total amount of circulating current.

The difference in the DC link voltages in phase $U$ is shown in Figure 3.27 by calculating the sum of the series-connected submodule voltages and subtracting the value of the parallelconnected ones. The value of the additional inductance in the output of each motor-connected cell in belts 1 and 3 was $L_{\mathrm{ser}}=1 \mu \mathrm{H}$ in the first simulation. In the second simulation, the value of the inductance was increased to $100 \mu \mathrm{H}$. With the higher inductance value, the circulating currents are effectively mitigated. The DC link voltage difference can be observed to fluctuate at an approximate frequency of $1.5 \mathrm{kHz}$. This is the switching frequency of one H-bridge cell on the grid and motor sides.

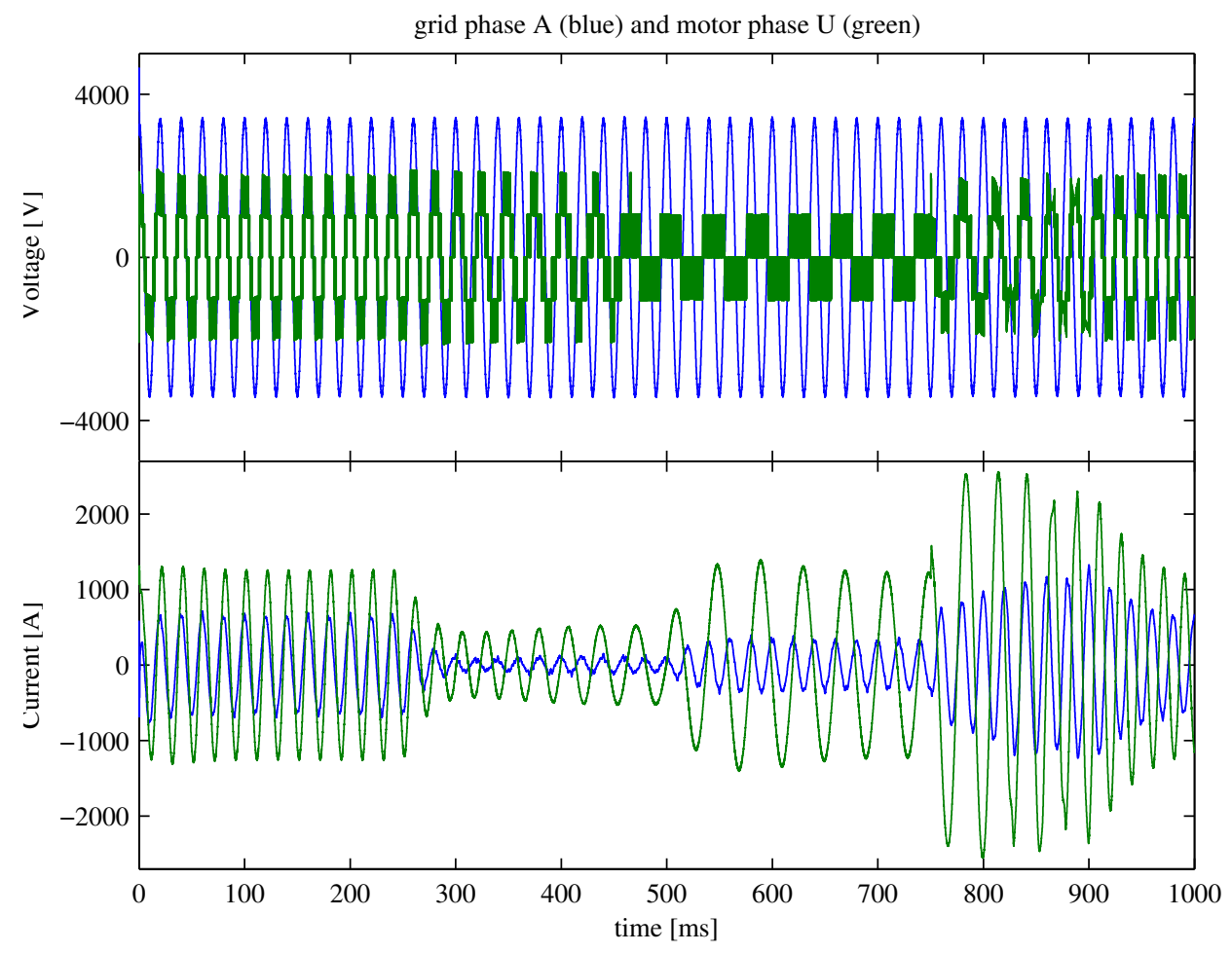

Figure 3.25 . $4.16 \mathrm{kV}$ grid voltage is transformed into $2.3 \mathrm{kV}$ by having four series-connected belts at the grid interface, and a combined series-parallel connection at the motor interface (two belts in series and two in parallel). The input and output voltages and currents are shown when the motor external torque is constant and the speed reference is varied. 


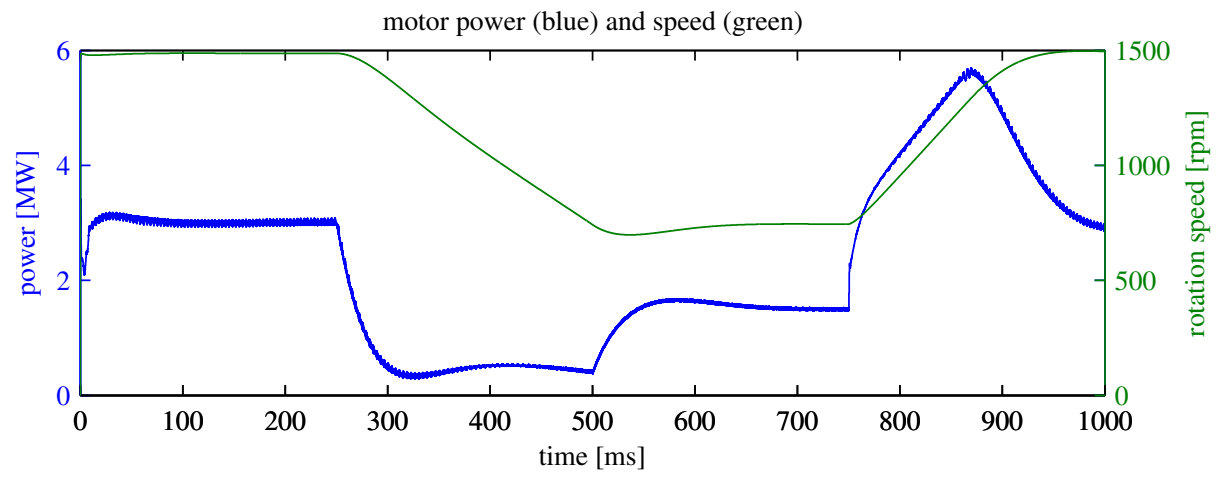

Figure 3.26. $4.16 \mathrm{kV}$ grid voltage is transformed into $2.3 \mathrm{kV}$. Four belts in series at the grid interface and a combined series-parallel connection on the motor side: two belts in series and two in parallel. The motor mechanical power and rotation speed are shown when the motor external torque is constant.
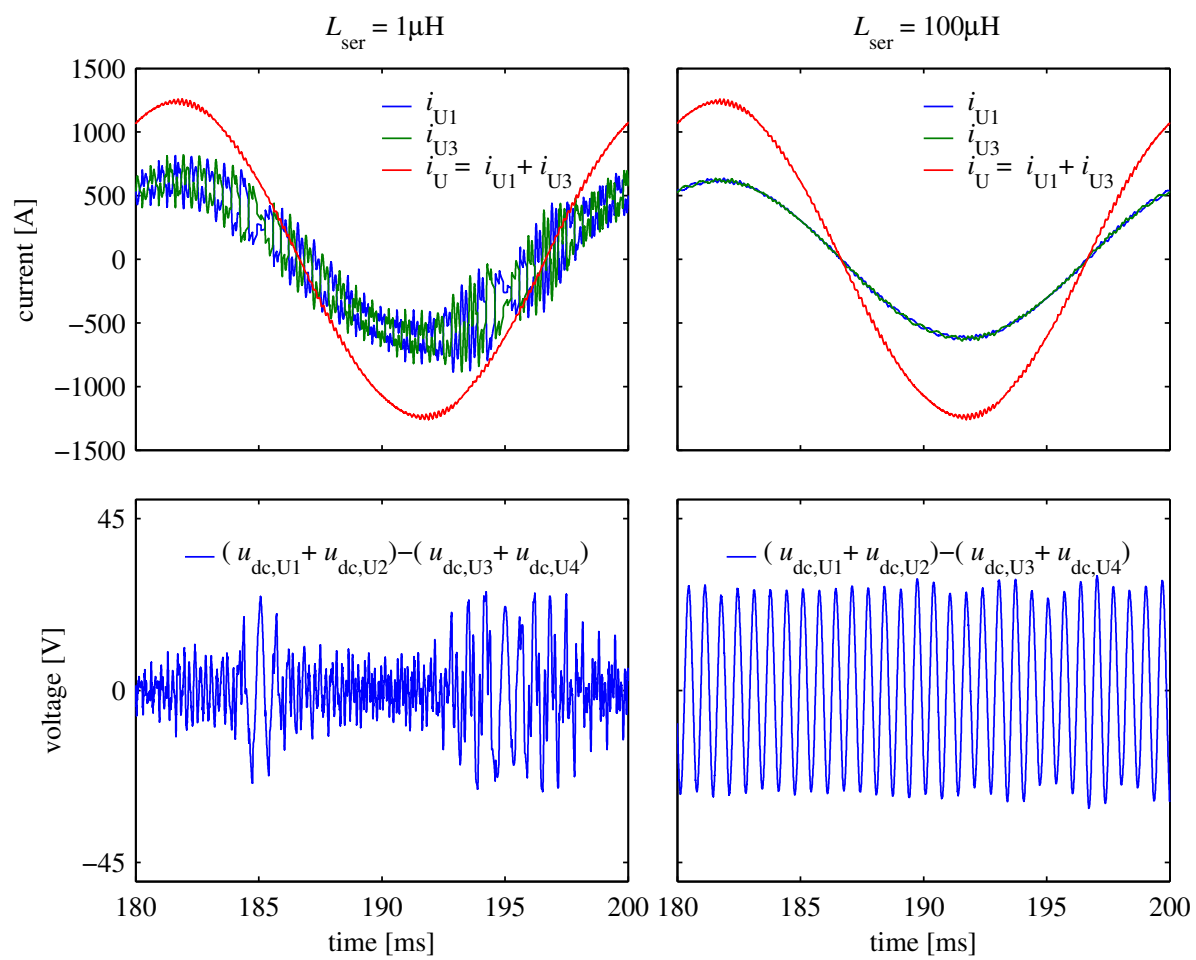

Figure 3.27 . $4.16 \mathrm{kV}$ grid voltage is transformed into $2.3 \mathrm{kV}$ by having four series-connected belts at the grid interface and a combined series-parallel connection at the motor interface (two belts in series and two in parallel). Circulating currents are generated by the fluctuating DC link voltages if the output series inductance is too low. 


\subsubsection{Delta connection of $\mathbf{H}$-bridges}

Another method to transform the voltage is to connect H-bridges in a delta configuration as shown in Figure 3.28. A voltage transformation ratio obtained by this configuration equals $\frac{1}{\sqrt{3}}=0.58$ when the higher voltage side is connected in star and the lower voltage side in delta. When the boosting effect of the AFE and the variable modulation index on the load side are taken into account, many different voltage transformation ratios are obtained.

In this configuration, the series-connected cells supply a line-to-line voltage to the load instead of a line-to-neutral. This means that the control algorithm has to be modified: the line-to-neutral voltage references $u_{i}^{*}$ are transformed into line-to-line voltage references with the following equations

$$
\begin{aligned}
& u_{\mathrm{UV}}^{*}=u_{\mathrm{U}}^{*}-u_{\mathrm{V}}^{*} \\
& u_{\mathrm{VW}}^{*}=u_{\mathrm{V}}^{*}-u_{\mathrm{W}}^{*} \\
& u_{\mathrm{WU}}^{*}=u_{\mathrm{W}}^{*}-u_{\mathrm{U}}^{*}
\end{aligned}
$$

The modulator does not require any modifications, but a relatively high-value inductor is needed in series with the phase outputs to limit the current rate of change resulting from the modulation in different phases. The cells in Figure 3.28 are intentionally drawn around the load in a circle to visualize the path of the circulating current produced by the delta connection. Suppose that at a certain time instant, cells in phase UV produce a voltage $u_{\mathrm{dc}}$, and at the same time, phases VW and WU produce zero volts. In this case, the cells in phase UV are directly shorted by the cells in the other phases, and without an inductor in the path of the short-circuit current, a failure will occur resulting in the destruction of multiple IGBTs and peripheral devices. The inductor value is determined by the highest voltage difference,

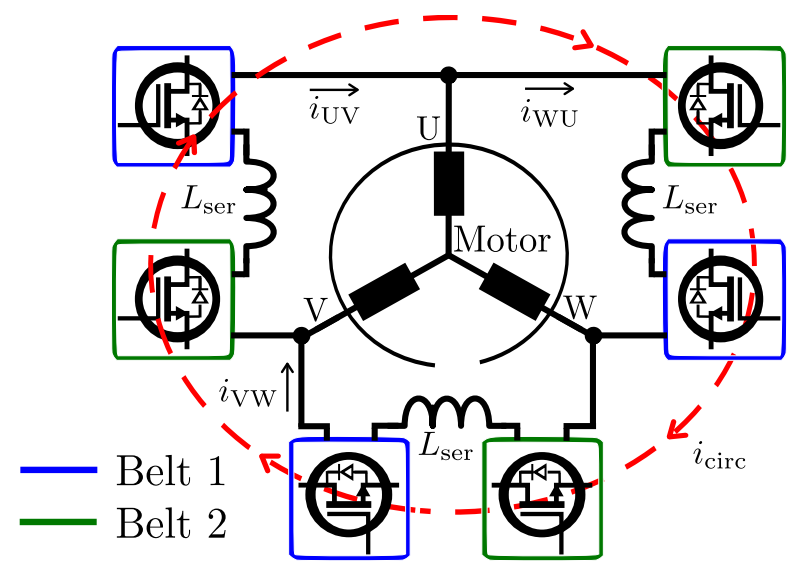

Figure 3.28. H-bridge cells of two belts are in a series-delta connection to drive the motor load. The colored borders of the H-bridge cells indicate the corresponding belt. The circulating current is reduced by the additional series reactors. The circulating current path is depicted by the red dashed line. 


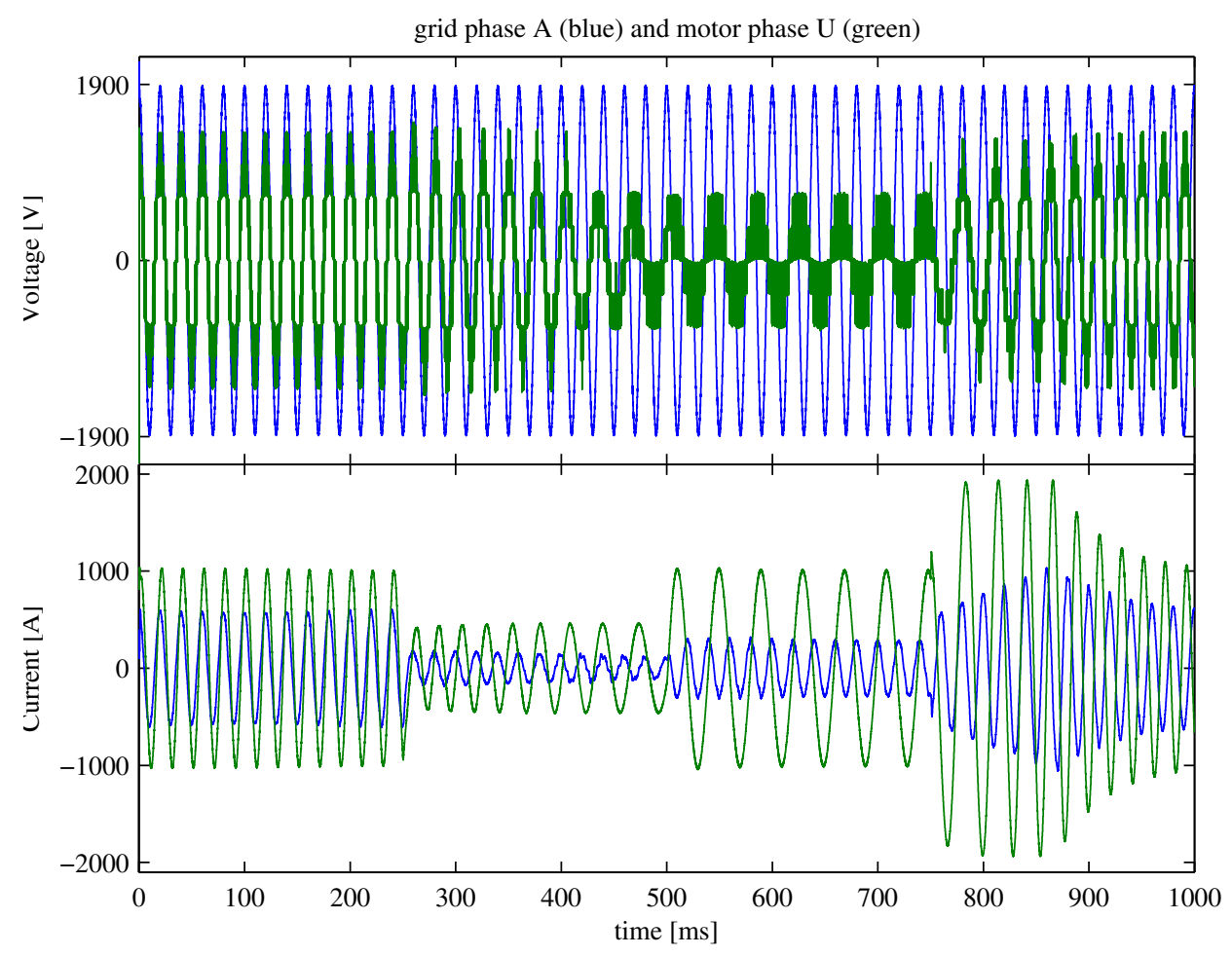

Figure $3.29 .2 .3 \mathrm{kV}$ grid voltage is transformed into $1.4 \mathrm{kV}$. Two belts in series at the grid and load interfaces, but a delta connection is used on the load side. The input and output voltages and currents are shown when the motor external torque is constant and the speed reference is varied.

the longest duration of one switching combination, and the maximum allowable intantaneous value of the circulating current. Thus, the value of inductance is proportional to the allowed circulating current and inversely proportional to the switching frequency.

The delta configuration was tested by a simulation by having two belts in series on the grid and motor sides. The motor side cells were connected in parallel so that the $2.3 \mathrm{kV}$ line-toline grid voltage was transformed to run a $1.4 \mathrm{kV}$ line-to-line motor. The series inductance value was chosen as $L_{\mathrm{ser}}=200 \mu \mathrm{H} \approx 0.053$ pu to suppress the circulating currents. The input and output waveforms are shown in Figure 3.29, and the motor mechanical speed and power in Figure 3.30. The figures show that the output voltage is approximately half and the current double of the input when the motor speed is nominal.

The currents in the first figure do not show the circulating currents because the measurement is located in series with the motor windings. The output currents of the two cells of belt 1 are shown with two different values of the series inductance $L_{\text {ser }}$ in Figure 3.31 in the nominal operating point of the motor. 


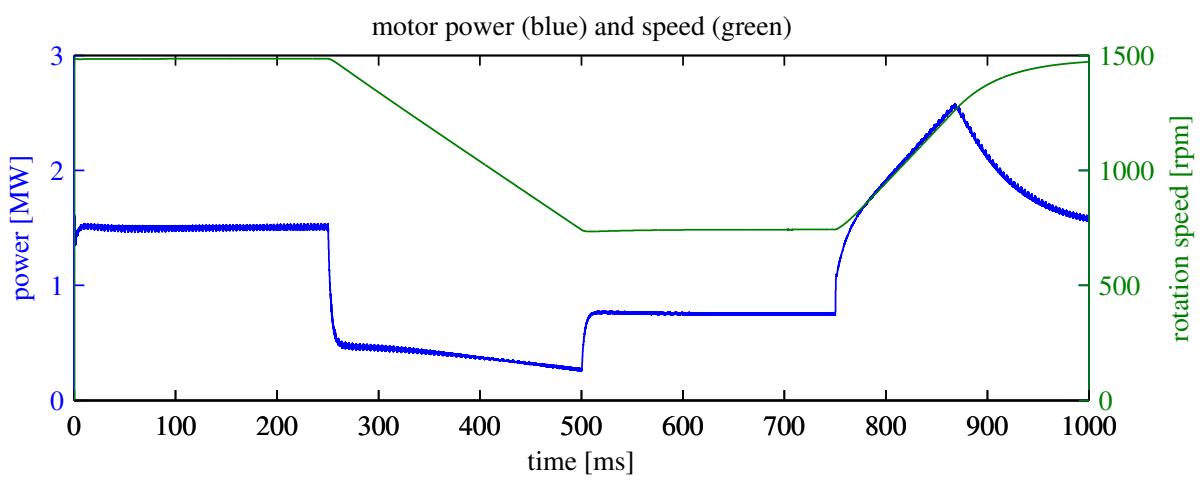

Figure 3.30. $2.3 \mathrm{kV}$ grid voltage is transformed into $1.4 \mathrm{kV}$. Two belts in series at the grid and load interfaces, but a delta connection is used on the load side. The motor mechanical power and rotation speed are shown when the motor external torque is constant.

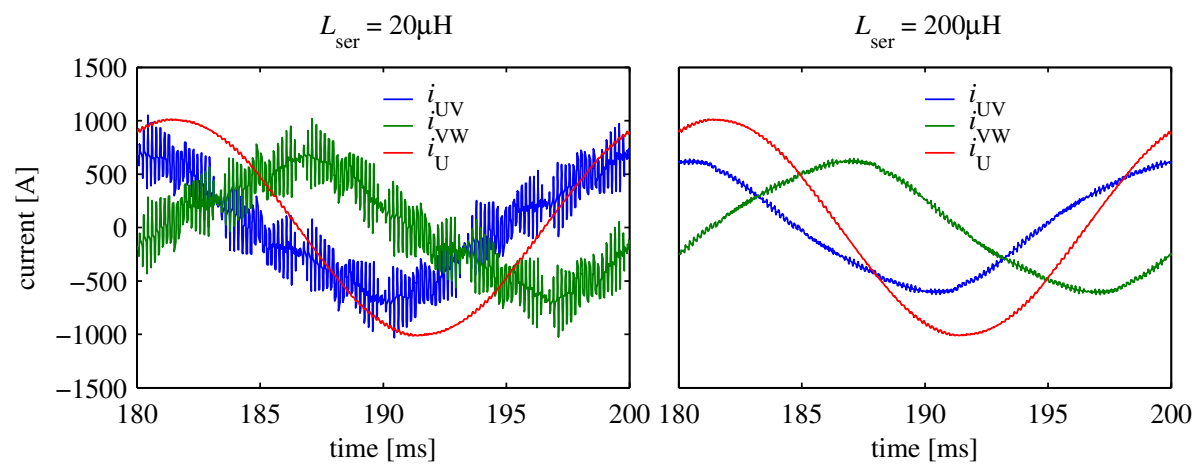

Figure $3.31 .2 .3 \mathrm{kV}$ grid voltage is transformed into $1.4 \mathrm{kV}$. Two belts in series at the grid and load interfaces, but a delta connection is used on the load side. The output current of one phase in belt 1 are shown together with the motor phase current. The effect of $L_{\mathrm{ser}}$ on the circulating currents is visible.

It can be concluded from the presented simulation results that a delta configuration is technically possible. A downside of the delta connection is the relatively large inductor needed to mitigate the circulating currents. The circulating currents are produced when cells in one phase are shorted by cells in the other phases and, as such, the nature of the circulating currents differs significantly from the parallel configuration.

\subsubsection{Multiport operation}

Using only the control algorithms presented before, the MDC can operate in a multiport application, yet on a limited scale. It is necessary to use the assisted DC link voltage controller (see Section 3.4.4) to balance the DC link voltages between the belts. In the following ex- 


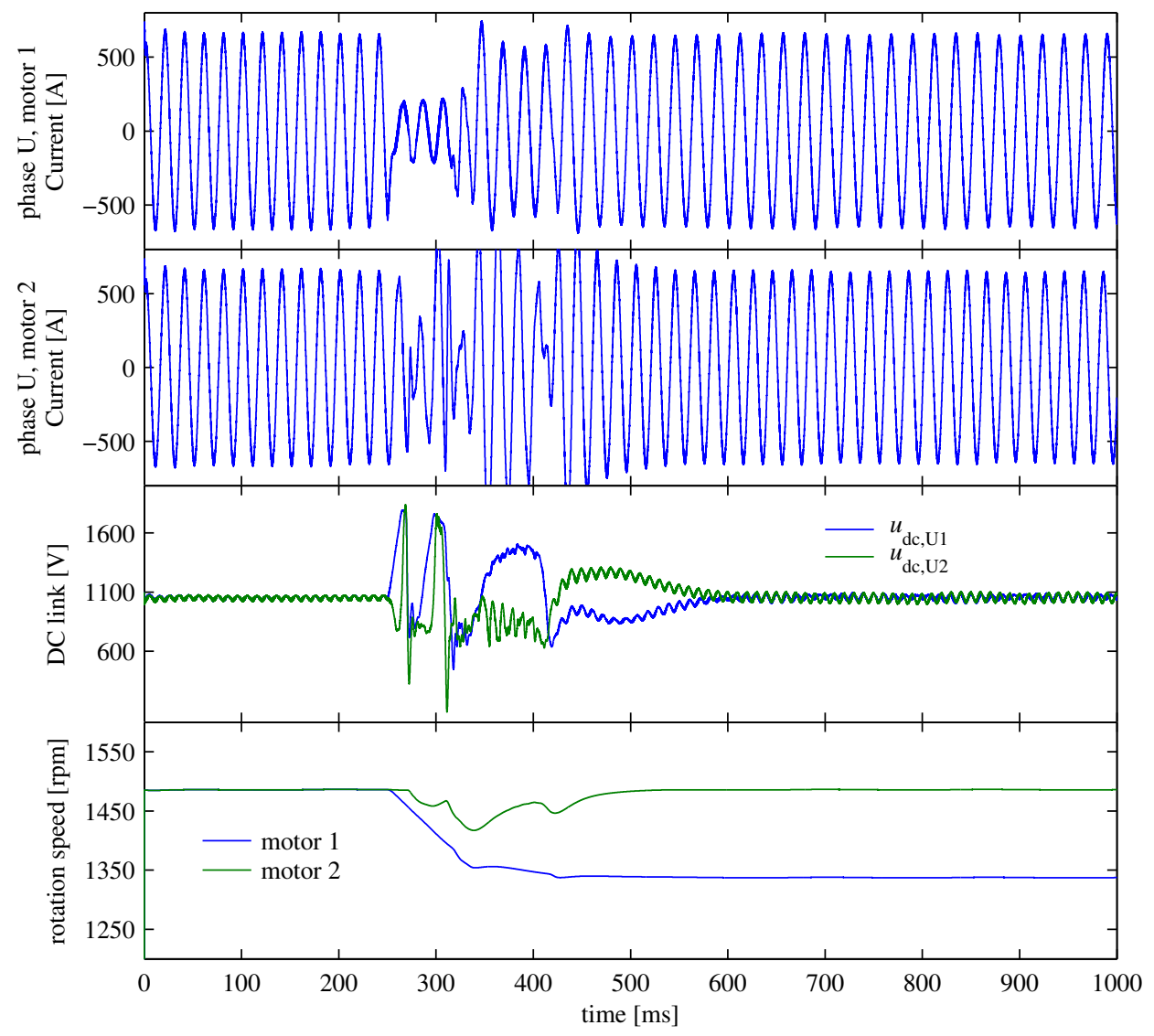

Figure $3.32 .2 .3 \mathrm{kV}$ grid voltage is transformed into two independent $1.1 \mathrm{kV}$ supplies. Two belts in series on the grid side and one belt per motor on the load side. A nominal external torque is applied to both motors throughout the simulation.

ample, a three-port application is simulated. The grid voltage is $2.3 \mathrm{kV}$ and two belts are connected in series by a wye configuration. Both belts have independent motor loads with a $1.1 \mathrm{kV}$ nominal line-to-line voltage and a nominal power of $750 \mathrm{~kW}$.

The simulation (Figure 3.32) starts with equal output characteristics of the two identical motors: nominal torque at nominal speed. Motor 1 is then decelerated to a $90 \%$ speed, and thus, its power is also reduced. To compensate for this, the assisted DC link voltage controller has to adjust the modulation indices of the series-connected cells on the grid side. After the transient, a balanced operation is achieved with different powers of the motors. However, the DC link voltages have ripple values during the transient that would result in a fatal malfunctioning in practice. The transient operation in a multiport application is a problem left outside the scope of this work. 
The performance of the MDC in a multiport application can be improved by adopting a control method from the UNIFLEX project. The voltage vector commanded by the AFE controller is decomposed into two components (in the case of two output ports in the system), with the sum voltage equaling the AFE controller output, but the components take into account the power in the two output ports (UNIFLEX-PM, 2007).

Regardless of the incomplete development of the controllers needed for multiport operation, the simulation result shows that multiport operation is possible. The limits of the operation, that is, the maximum power difference between the ports and the transient performance, are unknown for now. These questions are left for future research.

\subsection{Medium-frequency transformer link}

The true innovation of the MDC topology is the medium-frequency transformer link. The transformer not only provides modularity to the system, but also isolation of the supply and the load and an efficient and easy-to-control energy link between the input and output phases. The details of transformer link operation, control strategies, and power cell alternatives are presented in this section.

The circuit diagram of the MDC shows a transformer with three primary-side windings and three secondary-side windings. One could easily misinterpret it as a three-phase transformer. In reality, the construction of the transformer is similar to a single-phase transformer, where instantaneous, average, and RMS values of magnetic flux are equal among the windings. Also the number of winding turns are equal in different windings making the transformation ratio equal to one. In practice, this type of a transformer can be constructed either with an Eshaped core or a toroidal core. Further details of the physical construction of the transformer cannot be disclosed here because of IP issues.

In the previous sections, the input and output port configurations were studied, and practically no remarks were made on the transformer link. Actually, all simulations were performed with a symmetric transformer. The symmetry of the transformer is a critical design aspect since the power flow through the transformer is not controlled. Instead, the energy flow is naturally produced by the voltage differences in submodules around the transformer. If there are impedance variations in different energy flow paths, voltage differences persist between parallel submodules.

The natural energy flow through the transformer is obtained when the voltages of all windings are synchronous. This is obtained by controlling all H-bridges with equal and synchronous gate pulses. Figure 3.33 shows the gate pulses and output voltage of one H-bridge. The output voltage is a rectangular wave with a $50 \%$ pulsewidth, which means that a negative voltage pulse is supplied to the transformer for half of the cycle and a positive one for the other half. The frequency of the H-bridge output voltage equals the switching frequency of a single IGBT. Fundamentally, the output current of the H-bridge is produced by the voltage difference of the supplying and receiving submodules. The voltage difference is applied over 
the leakage impedance of the transformer, which is dominated by the leakage inductance. The current is the integral of the voltage difference, making its absolute value increase with an almost linear ramp during the half cycles. When the conducting IGBTs are turned off, the current is at its peak value and starts descending towards zero during the dead time, which prevents the short circuit of an H-bridge leg. Ideally, the current shifts to the antiparallel diodes instantly, and the polarity of the voltage is changed at the beginning of the dead time.

A simplified equivalent circuit of the six-winding medium-frequency transformer is shown in Figure 3.34a. The symmetry of the transformer can be seen from this figure: if the stray inductances and the winding resistances are equal in each winding, the couplings of the windings are equal. The roles of the windings are interchangeable meaning that any winding can be used for any phase in the primary or in the secondary. It also means that energy can flow from any winding to any other winding: the only variable affecting the amount of energy transfer between two windings is the voltage difference of the submodules of the two windings. The equivalent circuit of Figure $3.34 \mathrm{~b}$ corresponds better to the transformer implementation in the simulations. The inductances of a six-winding transformer can be described with a $6 \times 6$ matrix where nondiagonal elements represent the mutual inductances between windings. For example, the value in the $2^{\text {nd }}$ row $(i=2)$ and the $4^{\text {th }}$ column $(j=4)$ describes the mutual inductance from winding 2 to winding 4 . This value equals the value in the cell $i, j=4,2$, which is the mutual inductance from winding 4 to winding 2 . The mutual inductance matrix is therefore always symmetric, but that does not mean that the transformer is; the transformer is considered symmetric only if all nondiagonal elements of the mutual in-

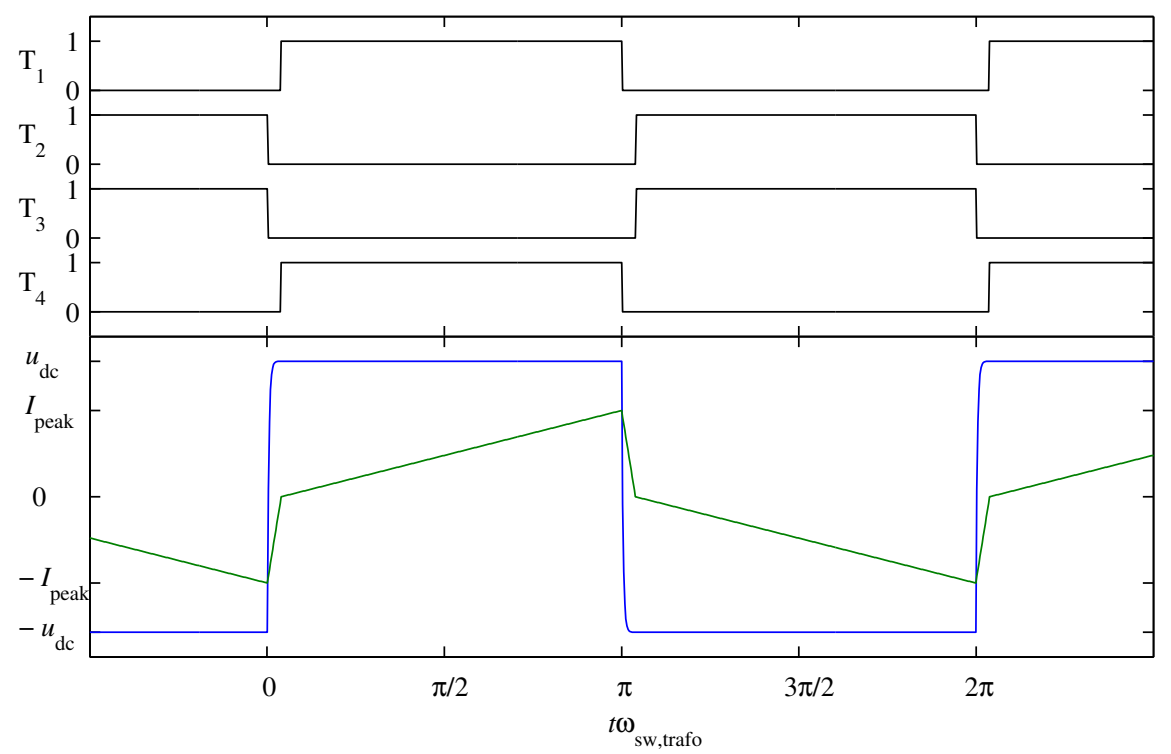

Figure 3.33. Gate pulses of transistors 1-4, output voltage, and output current of an H-bridge on the primary side of the transformer. IGBTs 1 and 4 conduct during the positive half cycle and 2 and 3 during negative. In normal operation, the six H-bridges are switched synchronously. 
ductance matrix are equal. The diagonal elements are winding self-inductances, that is, the magnetizing inductance of the transformer and, as the turns ratio is one, the diagonal elements are equal.

As an example of an asymmetric transformer, a toroidal core is considered with the six windings wound around the core with a constant spacing between adjacent windings. In this configuration, the distance from one winding to another is not the same between each winding, and the leakage flux paths differ, leading to unequal couplings. The design of a symmetric transformer is a challenging task and is omitted from the analysis here.

The mutual inductance matrix can be formed as a combination of two matrices. All elements of the first matrix $\mathbf{L}_{\mathrm{m}}$ equal the value of the transformer magnetizing inductance $L_{\mathrm{m}}$ and the other matrix $\mathbf{L}_{\sigma}$ is a symmetric matrix with the leakage inductance values. All values of the diagonal elements are zero in $\mathbf{L}_{\sigma}$. The mutual inductance matrix is defined as follows

$$
\mathbf{M}=\mathbf{L}_{\mathrm{m}}-\mathbf{L}_{\sigma}=\left[\begin{array}{cccccc}
L_{\mathrm{m}} & L_{\mathrm{m}} & L_{\mathrm{m}} & L_{\mathrm{m}} & L_{\mathrm{m}} & L_{\mathrm{m}} \\
L_{\mathrm{m}} & L_{\mathrm{m}} & L_{\mathrm{m}} & L_{\mathrm{m}} & L_{\mathrm{m}} & L_{\mathrm{m}} \\
L_{\mathrm{m}} & L_{\mathrm{m}} & L_{\mathrm{m}} & L_{\mathrm{m}} & L_{\mathrm{m}} & L_{\mathrm{m}} \\
L_{\mathrm{m}} & L_{\mathrm{m}} & L_{\mathrm{m}} & L_{\mathrm{m}} & L_{\mathrm{m}} & L_{\mathrm{m}} \\
L_{\mathrm{m}} & L_{\mathrm{m}} & L_{\mathrm{m}} & L_{\mathrm{m}} & L_{\mathrm{m}} & L_{\mathrm{m}} \\
L_{\mathrm{m}} & L_{\mathrm{m}} & L_{\mathrm{m}} & L_{\mathrm{m}} & L_{\mathrm{m}} & L_{\mathrm{m}}
\end{array}\right]-
$$

A corresponding $6 \times 6$ matrix can be defined for the winding resistances $\mathbf{R}_{\mathrm{w}}$. In a transformer with a symmetric winding configuration, the diagonal elements (winding self-resistances) can be assumed equal. The nondiagonal elements, the mutual resistances, are also assumed equal. The voltage drop over the transformer is dominated by the leakage inductance, not the

a)

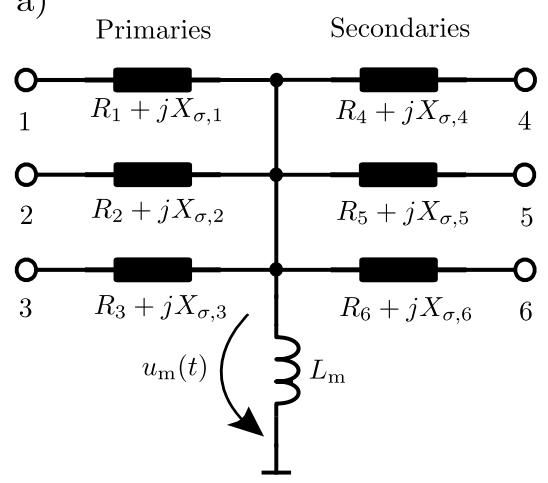

b)

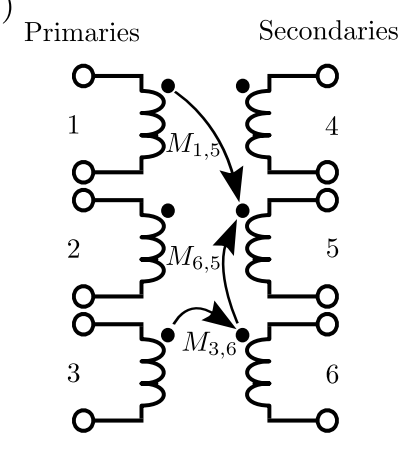

Figure 3.34. Two equivalent circuit depictions of the six-winding medium-frequency transformer. 
resistance of the windings.

This section includes various simulations, and comparisons are made with symmetric and nonsymmetric transformers. When a symmetric transformer is used, all nondiagonal elements of $\mathbf{L}_{\sigma}$ are set to $1.32 \mu \mathrm{H}$. With an asymmetric transformer, the following leakage inductance matrix is used. The average leakage inductance of the asymmetric transformer equals the leakage inductances of the symmetric transformer.

$$
\mathbf{L}_{\sigma}=\left[\begin{array}{cccccc}
0 & 1.09 & 1.70 & 1.06 & 1.32 & 1.89 \\
1.09 & 0 & 1.09 & 1.32 & 1.05 & 1.32 \\
1.70 & 1.09 & 0 & 1.89 & 1.32 & 1.06 \\
1.06 & 1.32 & 1.89 & 0 & 1.05 & 1.66 \\
1.32 & 1.05 & 1.32 & 1.05 & 0 & 1.05 \\
1.89 & 1.32 & 1.06 & 1.66 & 1.05 & 0
\end{array}\right] \times 10^{-6}[\mathrm{H}]
$$

The magnetizing inductance is $L_{\mathrm{m}}=500 \mu \mathrm{H}$ with both transformer configurations. The mutual resistances are set to $0 \Omega$ and the self-resistances to $2.6 \mathrm{~m} \Omega$ in the simulations.

Three methods to control the transformer-connected H-bridges were presented in (Korhonen et al., 2013a). In the first control scheme, all primary-side bridges are switched synchronously at the operating frequency of the transformer. In the second scheme, also the secondaryside bridges are switched correspondingly with the primary side, and in the third switching scheme (referred to as power direction control), a secondary-side bridge is switched only when the output power of the phase is negative. The second scheme, where all $\mathrm{H}$-bridges are switched synchronously, has been used throughout the dissertation so far, and will be used unless otherwise noted.

In the literature on push-pull-type bridges that feed medium or high-frequency transformers, flux walking and saturation have been described as problematic issues; see for example (Ortiz et al., 2011) and (Ortiz et al., 2014). In the research program on the MDC, a simulation-based preliminary study was conducted regarding the prevention of the transformer core saturation. The research was started in the fall 2010, and the author wrote his Master's thesis on the subject. In 2013, the control principle was granted a Finnish patent, see (Korhonen et al., 2013b). In 2014, the control method was published in (Sankala et al., 2014a). Further examination, such as experimental validation of the disclosed antisaturation control, has not been conducted in the course of the dissertation work.

\subsubsection{Comparison of transformer bridge cell topologies}

Three different transformer bridge cell configurations are reviewed in this section. The comparison is performed with simulations of a one-belt system, where the cells interfacing the transformer are varied. Submodule DC link voltage ripple and average values as well as winding current RMS and peak values are chosen as the variables that indicate the differences in the bridge cell configurations. Steady-state operation is considered. The parameters of the simulation model used in the bridge topology comparison are presented in Table 3.5. 
Figure 3.33 showed the conceptual voltage and current waveforms during normal operation when a normal H-bridge is used as the bridge cell. The current waveform indicates that the switch-off current is high, which produces excessive switching losses as the transformer operating frequency is several kilohertz. Two out of three cell topologies aim to reduce these switching losses by introducing resonance operation to the transformer link. The target of the resonance operation is to obtain a zero current switching (ZCS) behavior. The resonance operation also decreases the voltage drop over the transformer because leakage inductance is part of the resonance tank. The considered bridge topologies are presented in Figure 3.35.

\section{H-bridge cell with four IGBTs}

The first cell topology is the basic IGBT H-bridge, which comprises four transistors with antiparallel diodes. This is the topology that has been used in all the simulations presented earlier in this work. Fundamentally, the H-bridge is able to produce a three-level output voltage, the maximum and minimum values of which are $\pm u_{\mathrm{dc}}$. However, the zero voltage level is not employed in the supply voltage of the transformer. Winding current and DC link voltage waveforms with symmetric and asymmetric transformers are presented.

The winding currents can be observed to pulsate almost synchronously in Figure 3.36a. The

Table 3.5. Simulation study parameters of the cell topology comparison.

\begin{tabular}{llll}
\hline Element & Symbol & Value & Unit \\
\hline Grid frequency & $f_{\text {grid }}$ & 50 & $\mathrm{~Hz}$ \\
Grid line-to-line voltage & $U_{\mathrm{LL}}$ & 1100 & $\mathrm{~V}$ \\
Series-connected belts & $M$ & 1 & - \\
Switching frequency (grid\&load) & $f_{\mathrm{sw}, \mathrm{app}}$ & 6.0 & $\mathrm{kHz}$ \\
Transformer operating frequency & $f_{\mathrm{sw}, \mathrm{trafo}}$ & 18.0 & $\mathrm{kHz}$ \\
Dead time (all bridges) & $t_{\mathrm{dead}}$ & 2.0 & $\mu \mathrm{s}$ \\
Motor power (in steady state) & $P_{\mathrm{mot}}$ & 750 & $\mathrm{~kW}$ \\
Motor speed (in steady state) & $n_{\mathrm{mot}}$ & 1486 & $\mathrm{rpm}$ \\
DC link capacitance & $C_{\mathrm{dc}}$ & 1.0 & $\mathrm{mF}$ \\
Reference DC link voltage & $u_{\mathrm{dc}}^{*}$ & 1100 & $\mathrm{~V}$ \\
\hline
\end{tabular}

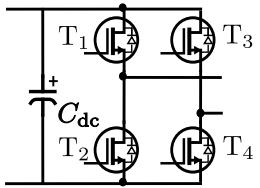

(a) H-bridge cell

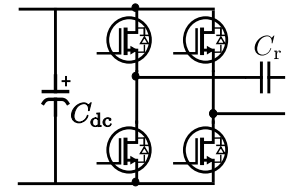

(b) H-bridge cell with an output capacitor

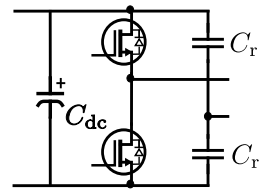

(c) Half bridge and a two-capacitor cell (HC bridge)

Figure 3.35. Three cell topologies considered in the comparison. 


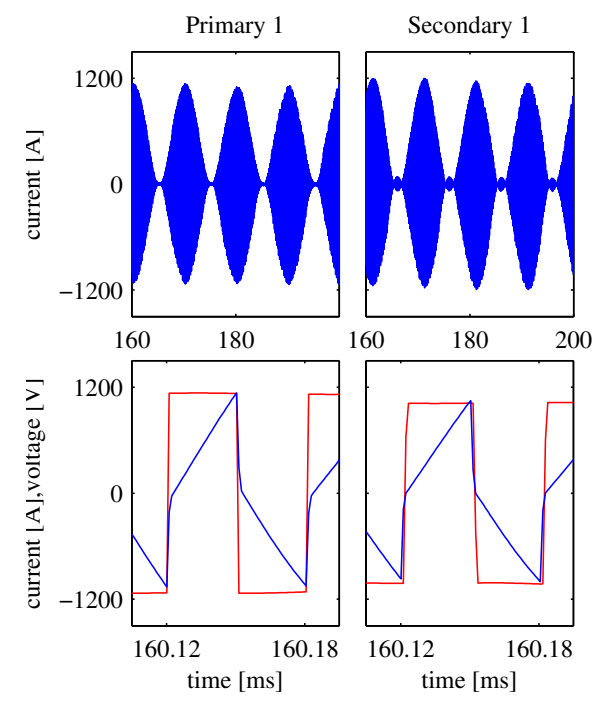

(a) Winding currents and voltages

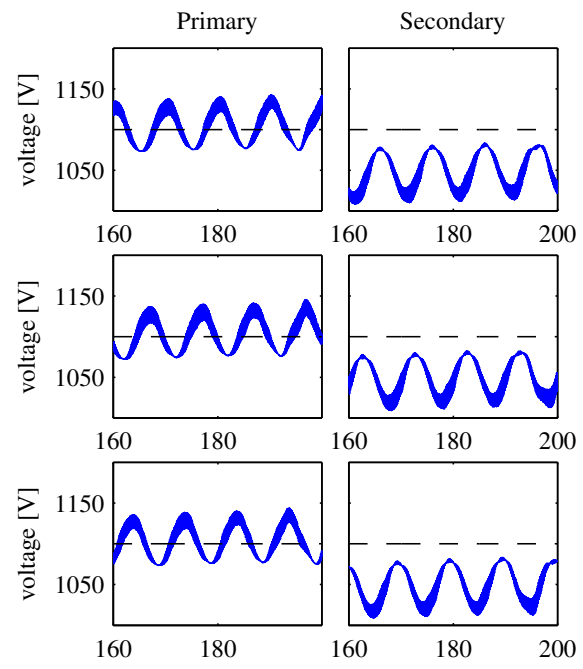

(b) DC link voltages

Figure 3.36. Steady-state operation with H-bridge cells interfacing the transformer.

motor is controlled to a $1486 \mathrm{rpm}$ speed by the motor controller, and with the nominal torque, the electrical frequency of the motor is very close to $50 \mathrm{~Hz}$ making the input and output seem synchronous at the presented time interval. The primary-side winding current peak value reaches $1.2 \mathrm{kA}$ while the RMS value is below $400 \mathrm{~A}$. Indeed, the frequency spectrum of the current includes harmonics of the transformer operating frequency, as can be seen in the enlargement in the figure. The dominant component at low frequencies is the double input/output frequency $(100 \mathrm{~Hz})$, which can be seen from the amplitude variation of the current. The same low-frequency ripple is seen in the DC link voltages, see Figure 3.36b. The $100 \mathrm{~Hz}$ fluctuation is in the phase opposition between the primary and secondary sides.

Figure 3.37 illustrates the effect of having a transformer with an asymmetric magnetic coupling of the submodules. No visible change can be observed in the winding currents, but the difference can be seen in the DC link voltages. The amplitude of double fundamental frequency fluctuation is clearly different between the submodules, and also the average values have slight variations: the primary-side voltage average values are $1103.0 \mathrm{~V}, 1104.1 \mathrm{~V}$, and 1103.4 V. The average values on the secondary side are $1041.2 \mathrm{~V}, 1041.2 \mathrm{~V}$, and $1041.6 \mathrm{~V}$. The differences in average values are negligibly small, but with the symmetric transformer, the average values were equal among all primary-side submodules and correspondingly equal among the secondary-side submodules. The difference in the average value will give rise to the circulating currents in the parallel connection of belts as was discussed in Section 3.5.2. 


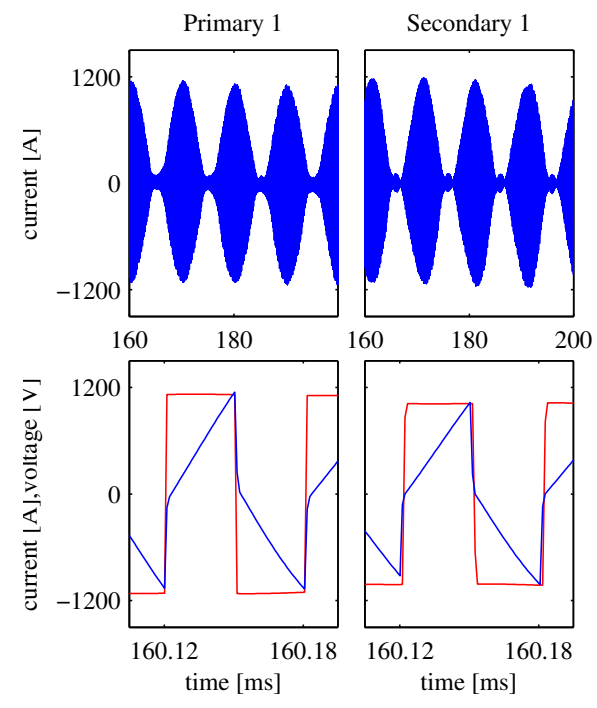

(a) Winding currents and voltages

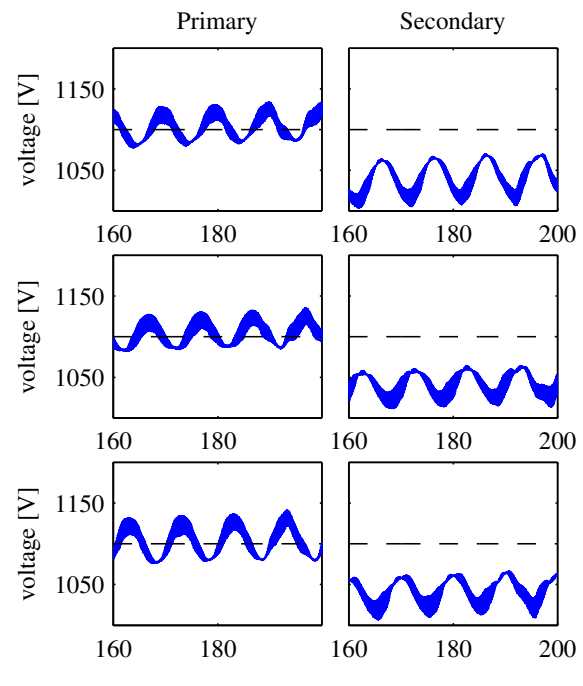

(b) DC link voltages

Figure 3.37. Steady-state operation with H-bridge cells interfacing the transformer. The asymmetric leakage inductance matrix creates variations between the DC link voltages.

\section{H-bridge cell with four IGBTs and an output capacitor}

The second cell topology considered in this comparative study is provided by adding a series capacitor to the output of a regular H-bridge. This topology was studied in detail in (Sankala et al., 2013). The purpose of the series capacitor is to act as part of a resonant tank tuned to the operation frequency of the transformer. The other, inductive, energy storage of the resonant tank is the leakage inductance of the transformer. At the resonance frequency, the reactance of the circuit goes to zero, and thus, the voltage and current are in phase. This means that the switch-off current of the IGBTs is significantly lower than in the hard-switching H-bridge cell topology. It was concluded in (Sankala et al., 2013), that true zero current switching is not obtained with this configuration because of the magnetizing current of the transformer and the component tolerances. The magnetizing current will always lag the voltage by 90 degrees because the magnetizing inductance $L_{\mathrm{m}}$ becomes part of the circuit. As $L_{\mathrm{m}}$ is usually a couple of decades greater than $L_{\sigma}$, the resonance is not obtained.

The capacitance of the added capacitor in each winding can be calculated when the operating frequency is known. As stated in (Sankala et al., 2013), the dead time of the H-bridges has also to be considered as it effectively decreases the conduction time of the IGBTs. With a zero dead time, the voltage transits from $u_{\mathrm{dc}}$ to $-u_{\mathrm{dc}}$ occur when transistors $\mathrm{T}_{1}$ and $\mathrm{T}_{4}$ are turned off and, at the same time, transistors $\mathrm{T}_{2}$ and $\mathrm{T}_{3}$ are turned on. With a perfect resonance, this is also the exact moment of the zero crossing of the current. When the dead time is nonzero, a short time period is waited before turning $\mathrm{T}_{2}$ and $\mathrm{T}_{3}$ on. In order to obtain ZCS operation, 
the current should be zero at the turn-off moment, but the positive half period of the current was started later than in an ideal case because of the dead time. Thus, the length of the half period is $t_{\mathrm{sw} \text {,trafo }} / 2-t_{\text {dead }}$. Knowing this, the capacitance can be defined as

$$
C_{\mathrm{r}}=\frac{1}{\left(2 \pi f_{\mathrm{r}}\right)^{2} L_{\mathrm{r}}}=\frac{1}{\left(2 \pi \frac{1}{t_{\mathrm{sw}, \text { trafo }}-2 t_{\text {dead }}}\right)^{2} L_{\mathrm{r}}},
$$

where $L_{\mathrm{r}}$ is the inductance in the resonance tank. If a symmetric transformer is used, $L_{\mathrm{r}}$ equals the leakage inductance. With an asymmetric transformer, the average leakage inductance must be calculated and substituted into the equation. If the leakage inductance matrix (3.49) is considered, the average value is $1.32 \mu \mathrm{H}$ and (3.50) gives a value of $51 \mu \mathrm{F}$ for the capacitor. The simulation results for this system are shown in Figure 3.38.

As a result of the resonance and nearly zero reactance of the circuit, the average of the DC link voltages on the secondary side are only $15 \mathrm{~V}$ lower than the primary-side voltages. The voltage drop is dictated by the on-time voltage drops of the IGBTs. The currents can be observed to behave almost sinusoidally, but the current zero crossing is not concurrent with the zero crossing of voltage, and thus, ZCS operation is not achieved. This is caused by the unequal magnetic coupling of the transformer.

As proposed in (Sankala et al., 2013), the asymmetry problem can be mitigated by adding an additional inductance with a higher value than the leakage in each winding. An additional inductance $L_{\mathrm{add}}=2 \mu \mathrm{H}$ was applied, and $C_{\mathrm{r}}$ was recalculated with $L_{\mathrm{r}}=3.32 \mu \mathrm{H}$. Then, the simulation result shown in Figure 3.39 was obtained. Now, the currents are closer to zero at the time of the switch-off compared with the previous simulation result. A downside is that the voltage drop over the transformer has been doubled.

The effect of tolerances in the values of $L_{\text {add }}$ and $C_{\mathrm{r}}$ on the performance of resonance was studied in (Sankala et al., 2013), and a detailed analysis is therefore omitted here. It was observed that, compared with the symmetric transformer and zero component tolerances, the switch-off current is doubled on the primary side and is unaffected on the secondary side when series inductances are added and component tolerances of $L_{\text {add }}$ and $C_{\mathrm{r}}$ of approximately $8 \%$ are applied.

\section{Half-bridge with two IGBTs and two capacitors}

The final bridge topology considered in the comparative study is provided by replacing the IGBTs of the other H-bridge leg with capacitors as shown in Figure 3.35c. This cell topology reduces the number of transistors inside a belt by 12 compared with the H-bridge cell. The capacitors can be dimensioned to obtain resonant operation and thereby reduce switching losses of the remaining IGBTs. The downside of the half bridge cell is that the supply voltage of the transformer is reduced to half, and the winding current is doubled as the power remains the same.

The winding current is always shared between the two capacitors, and therefore, the capacitance of the resonance circuit is formed by parallel connection of the two capacitors. Thus, 


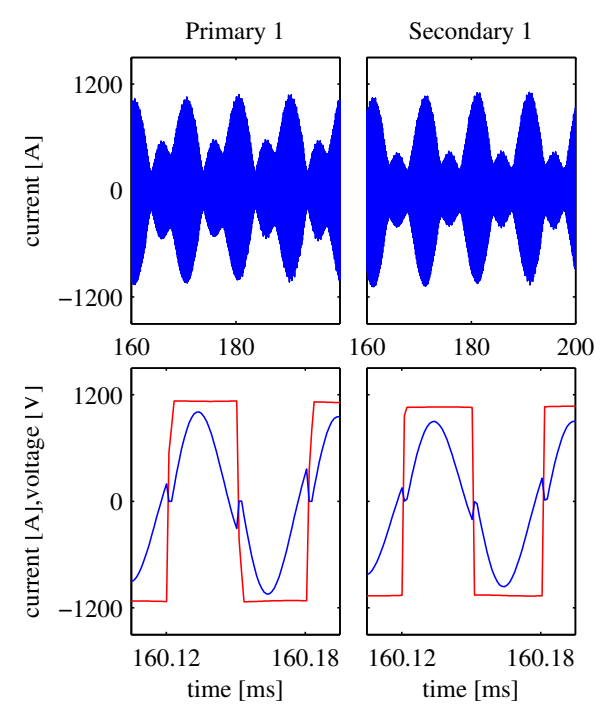

(a) Winding currents and voltages

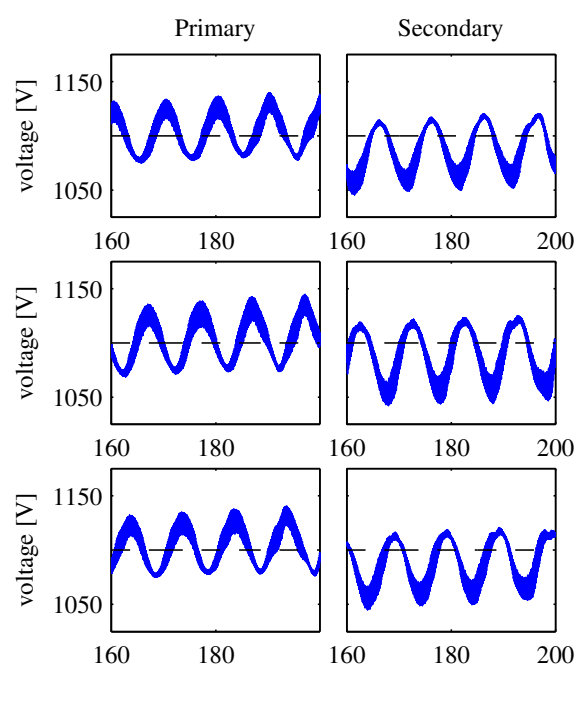

(b) DC link voltages

Figure 3.38. Steady-state operation with H-bridge cells with series capacitors interfacing the transformer. Resonance operation reduces the voltage drop to $15 \mathrm{~V}$.

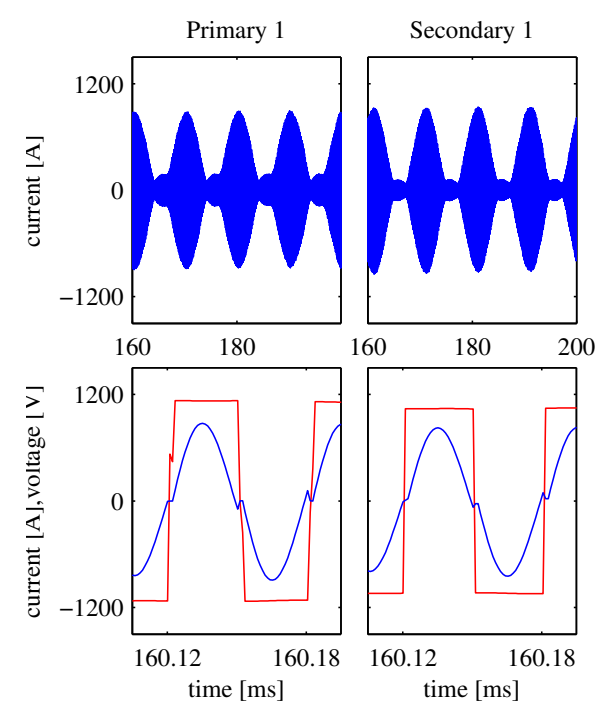

(a) Winding currents and voltages

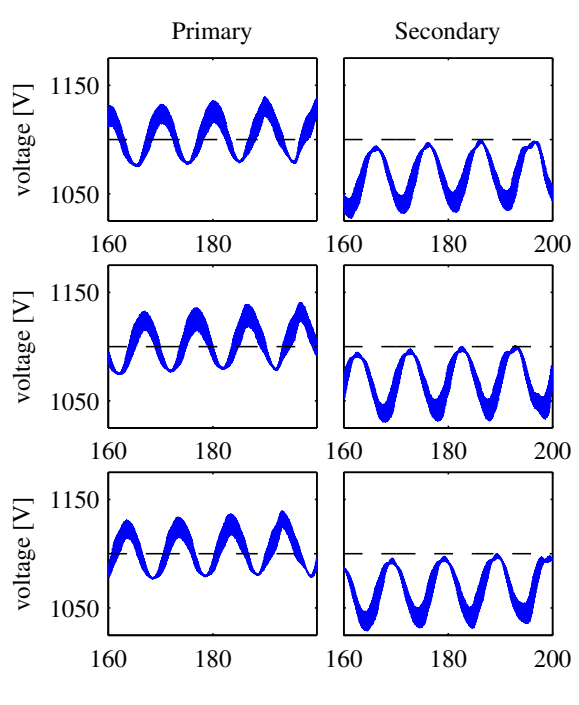

(b) DC link voltages

Figure 3.39. Steady-state operation with H-bridge cells with series capacitors interfacing the transformer. Asymmetric leakage inductances are compensated by an additional inductance. 


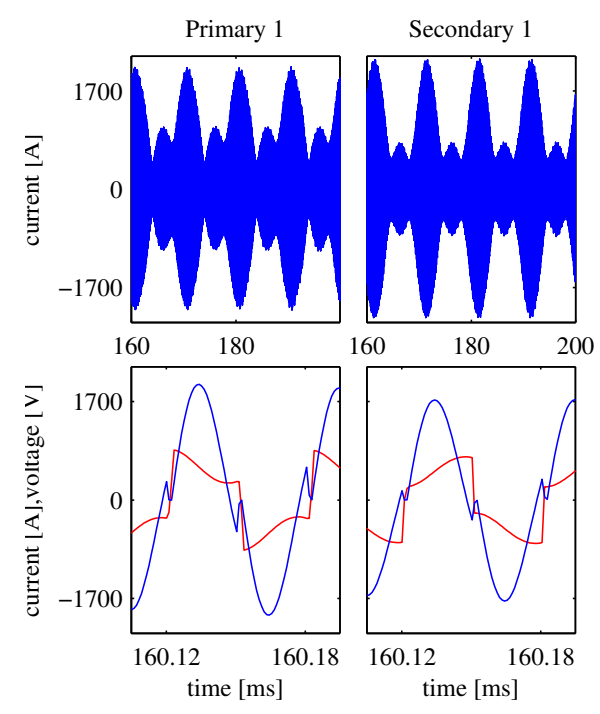

(a) Winding currents and voltages

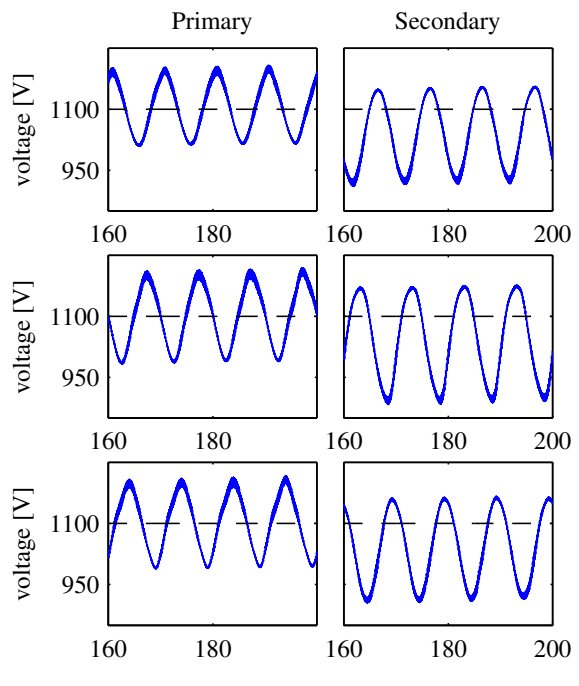

(b) DC link voltages

Figure 3.40. Steady-state operation with HC-bridge cells interfacing the transformer. The transformer current is high because of the lower winding voltage.

the capacitance of a single capacitor can be dimensioned by dividing the result of (3.50) by two.

Figure 3.40 shows the simulation result when an asymmetric transformer is used. The immediate observation from Figure 3.40a is the clearly higher amplitude of the winding currents; the peaks exceed $2500 \mathrm{~A}$. The winding voltages shown in the graph are measured from the output of the cell. Evidently, they no longer have a rectangular waveform as the voltage of the components of the resonant circuit produce distortion. The DC link voltages in Figure $3.40 \mathrm{~b}$ show a higher amplitude of oscillation at twice the fundamental frequency compared with the previous topologies.

\section{Comparison of bridge topologies}

In order to compare the three transformer bridge cell topologies, the simulation results shown previously were processed into a table showing the values of DC voltage ripple and voltage drop over the transformer and winding current peak and RMS values. The data are presented in Table 3.6. The DC voltage difference presented in the table is calculated as

$$
u_{\mathrm{dc}, \mathrm{diff}}=\frac{1}{3}\left(u_{\mathrm{dc}, \mathrm{avg}, \mathrm{A}}+u_{\mathrm{dc}, \mathrm{avg}, \mathrm{B}}+u_{\mathrm{dc}, \mathrm{avg}, \mathrm{C}}\right)-\frac{1}{3}\left(u_{\mathrm{dc}, \mathrm{avg}, \mathrm{U}}+u_{\mathrm{dc}, \mathrm{avg}, \mathrm{V}}+u_{\mathrm{dc}, \mathrm{avg}, \mathrm{W}}\right),
$$

where $u_{\mathrm{dc}, \mathrm{avg}, i}$ is the average value of the DC link voltage in phase $i$ calculated from the data points of a $40 \mathrm{~ms}$ time span. This means that the presented voltage difference is the average 
Table 3.6. Comparison of transformer bridge cell topologies.

\begin{tabular}{llcccc}
\hline $\begin{array}{l}\text { Cell } \\
\text { topology }\end{array}$ & $\begin{array}{l}\text { Transformer } \\
\text { setup }\end{array}$ & $\begin{array}{l}\text { DC volt- } \\
\text { age rip- } \\
\text { ple [V] }\end{array}$ & $\begin{array}{l}\text { DC voltage } \\
\text { difference } \\
{[\mathrm{V}]}\end{array}$ & $\begin{array}{l}\text { Winding } \\
\text { current } \\
\text { peak [A] }\end{array}$ & $\begin{array}{l}\text { Winding } \\
\text { current } \\
\text { RMS [A] }\end{array}$ \\
\hline H-bridge & symmetric & 74.5 & 53.9 & 1204 & 374 \\
& asymmetric & 66.3 & 62.2 & 1222 & 376 \\
H-bridge $+C_{\mathrm{r}}$ & symmetric & 44.7 & 15.5 & 979 & 350 \\
& asymmetric & 82.8 & 15.1 & 1255 & 426 \\
HC-bridge & asymmetric $+L_{\text {add }}$ & 71.8 & 33.8 & 973 & 366 \\
& symmetric & 89.0 & 61.6 & 1892 & 740 \\
& asymmetric & 294 & 62.1 & 2529 & 936 \\
\hline
\end{tabular}

voltage drop from the primary to the secondary.

When the DC link voltage ripple values are compared, the H-bridge cell with the resonance capacitor and the symmetric transformer stands out as the best alternative. The asymmetric transformer doubles the ripple voltage with this cell, but the ripple can be reduced by adding a compensating inductor $L_{\text {add }}$. While the added inductor increases the effective voltage drop, the peak values of winding currents are reduced.

The HC bridge has a feasible DC link voltage ripple with the symmetric transformer, but the ripple gets too high with the asymmetric transformer. With both transformer configurations, the HC bridge exhibits very high winding current values compared with the other cell topologies. With this cell, the switches must withstand a much higher current, and therefore, this cell topology is not considered beneficial to the MDC. Thus, the HC bridge is disregarded hereafter.

When the regular $\mathrm{H}$-bridge and the resonant $\mathrm{H}$-bridge are compared, the resonant topology is better in the light of data shown in the table. The asymmetric transformer poses problems with both cell topologies as the winding currents are not balanced, leading to differences in the ripple of the DC link voltages between submodules.

The final decision on which of the two topologies is better requires a more detailed study. As the resonance was applied in the hope of lower switching losses, a comparison of the efficiencies with and without a resonance capacitor is needed to verify the actual impact of the approach on the losses. Further research on these details is recommended.

\subsubsection{Analysis of the submodule current and voltage frequency spec- trum}

In the following, an approximate frequency spectrum analysis is made for the submodule voltages and currents. The analysis is based on the analysis presented in (Lezana et al., 2009). 
The target of the analysis is to establish a theoretical background for the different components in the frequency spectrum of the transformer winding currents and the submodule DC link voltages. The notation used in the analysis is explained in Figure 3.41.

The analysis is an iterative process, which starts from the assumption that the input voltage and current (= transformer winding voltage and current) are sinusoidal with the frequency $\omega_{\mathrm{i}}=2 \pi f_{\mathrm{sw} \text {,trafo }}$ and free of harmonic content. Similarly, the output voltage and current $(=$ motor/grid voltage and current) are approximated to comprise one frequency component, $\omega_{0}$. The DC link voltage is solved and the input current is recalculated as a modulation of the DC link input current $i_{2}$ and the transformer operating frequency. Thus, the initial assumption of the sinusoidal current is updated with inclusion of other relevant frequency components.

Let us consider the output power of a single submodule on the load side. Neglecting switching harmonics, the output voltage and current can be considered sinusoidal

$$
\begin{aligned}
u_{\mathrm{o}}(t) & =\hat{u}_{\mathrm{o}} \sin \left(\omega_{\mathrm{o}} t+\varphi_{\mathrm{o}}\right) \\
i_{\mathrm{o}}(t) & =\hat{i}_{\mathrm{o}} \sin \left(\omega_{\mathrm{o}} t\right) .
\end{aligned}
$$

Consequently, the instantaneous output power can be written as

$$
p_{\mathrm{o}}(t)=\hat{u}_{\mathrm{o}} \hat{i}_{\mathrm{o}} \sin \left(\omega_{\mathrm{o}} t+\varphi_{\mathrm{o}}\right) \sin \left(\omega_{\mathrm{o}} t\right)=\frac{\hat{u}_{\mathrm{o}} \hat{i}_{\mathrm{o}}}{2}\left[\cos \varphi_{\mathrm{o}}-\cos \left(2 \omega_{\mathrm{o}} t+\varphi_{\mathrm{o}}\right)\right]
$$

Thus, the power contains a component with twice the output frequency and the DC component. The output power can also be written using the output current from the DC link

$$
p_{\mathrm{o}}(t)=u_{\mathrm{dc}}(t) i_{1}(t)
$$

The DC link output current is then

$$
i_{1}(t)=\frac{\hat{u}_{\mathrm{o}} \hat{i}_{\mathrm{o}}}{2 u_{\mathrm{dc}}(t)}\left[\cos \varphi_{\mathrm{o}}-\cos \left(2 \omega_{\mathrm{o}} t+\varphi_{\mathrm{o}}\right)\right] .
$$

Let us now consider the DC link voltage of a submodule. The current of the capacitor can be written as

$$
i_{\mathrm{c}}(t)=C_{\mathrm{dc}} \frac{\mathrm{d} u_{\mathrm{dc}}(t)}{\mathrm{d} t}
$$

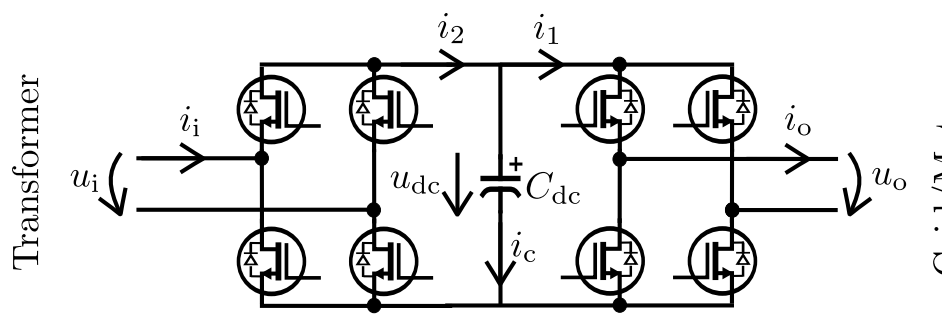

Figure 3.41. Circuit diagram of a submodule. 
Considering that

$$
\frac{\mathrm{d} u_{\mathrm{dc}}^{2}(t)}{\mathrm{d} t}=2 u_{\mathrm{dc}}(t) \frac{\mathrm{d} u_{\mathrm{dc}}(t)}{\mathrm{d} t}
$$

or

$$
\frac{\mathrm{d} u_{\mathrm{dc}}(t)}{\mathrm{d} t}=\frac{1}{2 u_{\mathrm{dc}}(t)} \frac{\mathrm{d} u_{\mathrm{dc}}^{2}(t)}{\mathrm{d} t}
$$

the expression of the current can be changed to

$$
i_{\mathrm{c}}(t)=\frac{C_{\mathrm{dc}}}{2 u_{\mathrm{dc}}(t)} \frac{\mathrm{d} u_{\mathrm{dc}}^{2}(t)}{\mathrm{d} t}
$$

The instantaneous power of the DC link capacitor is then

$$
p_{\mathrm{c}}(t)=u_{\mathrm{dc}}(t) i_{\mathrm{c}}(t)=\frac{C_{\mathrm{dc}}}{2} \frac{\mathrm{d} u_{\mathrm{dc}}^{2}(t)}{\mathrm{d} t} .
$$

From the instantaneous power equation, the capacitor voltage can be written as

$$
u_{\mathrm{dc}}(t)=\sqrt{\frac{2}{C_{\mathrm{dc}}} \int_{0}^{t} p_{\mathrm{c}}(\tau) \mathrm{d} \tau+u_{\mathrm{dc}}^{2}(0)},
$$

where $u_{\mathrm{dc}}(0)$ is the average value of the DC link voltage in the steady state. Next, the voltage of the capacitor is expressed as its Taylor expansion. The Taylor expansion of a function can be written as

$$
f(x)=f(0)+\frac{f^{\prime}(0)}{1 !} x+\frac{f^{\prime \prime}(0)}{2 !} x^{2}+\ldots
$$

Using only the first derivative, the voltage can be written as

$$
u_{\mathrm{dc}}(t) \approx u_{\mathrm{dc}}(0)+\frac{1}{u_{\mathrm{dc}}(0)} \frac{1}{C_{\mathrm{dc}}} \int_{0}^{t} p_{\mathrm{c}}(\tau) \mathrm{d} \tau
$$

The capacitor instantaneous power is the difference between the submodule input and output powers

$$
p_{\mathrm{c}}(t)=p_{\mathrm{i}}(t)-p_{\mathrm{o}}(t)
$$

In order to keep the analysis simple, the input power (power from the transformer) is approximated to contain only the first harmonics of current and voltage, and the magnetizing current of the transformer is disregarded.

$$
p_{\mathrm{i}}(t)=\hat{u}_{\mathrm{i}} \hat{i}_{\mathrm{i}} \sin \left(\omega_{\mathrm{i}} t+\varphi_{\mathrm{i}}\right) \sin \left(\omega_{\mathrm{i}} t\right)=\frac{\hat{u}_{\mathrm{i}} \hat{\mathrm{i}}_{\mathrm{i}}}{2}\left[\cos \varphi_{\mathrm{i}}-\cos \left(2 \omega_{\mathrm{i}} t+\varphi_{\mathrm{i}}\right)\right] .
$$

In the steady state, the DC component terms in the input and output powers must be equal, otherwise the average DC link voltage will rise.

$$
p_{\mathrm{C}}(t)=\frac{\hat{u}_{\mathrm{o}} \hat{i}_{\mathrm{o}}}{2} \cos \left(2 \omega_{\mathrm{o}} t+\varphi_{\mathrm{o}}\right)-\frac{\hat{u}_{\mathrm{i}} \hat{\mathrm{i}}_{\mathrm{i}}}{2} \cos \left(2 \omega_{\mathrm{i}} t+\varphi_{\mathrm{i}}\right)
$$

Substituting (3.67) into (3.64) yields

$$
u_{\mathrm{dc}}(t) \approx u_{\mathrm{dc}}(0)+\frac{1}{C_{\mathrm{dc}} u_{\mathrm{dc}}(0)} \int_{0}^{t}\left[\frac{\hat{u}_{\mathrm{o}} \hat{i}_{\mathrm{o}}}{2} \cos \left(2 \omega_{\mathrm{o}} \tau+\varphi_{\mathrm{o}}\right)-\frac{\hat{u}_{\mathrm{i}} \hat{\mathrm{i}}_{\mathrm{i}}}{2} \cos \left(2 \omega_{\mathrm{i}} \tau+\varphi_{\mathrm{i}}\right)\right] \mathrm{d} \tau .
$$


Solving the integral of (3.68) and reorganizing the terms gives the following expression

$$
u_{\mathrm{dc}}(t) \approx u_{\mathrm{dc}}(0)-\frac{\hat{u}_{\mathrm{i}} \hat{i}_{\mathrm{i}}}{4 C_{\mathrm{dc}} u_{\mathrm{dc}}(0)} \sin \left(2 \omega_{\mathrm{i}} t+\varphi_{\mathrm{i}}\right)+\frac{\hat{u}_{\mathrm{o}} \hat{i}_{\mathrm{o}}}{4 C_{\mathrm{dc}} u_{\mathrm{dc}}(0)} \sin \left(2 \omega_{\mathrm{o}} t+\varphi_{\mathrm{o}}\right) .
$$

Based on the above, the current to the DC link can be considered to contain a DC component and components on $2 \omega_{\mathrm{o}}$ and $2 \omega_{\mathrm{i}}$ and thus, the following expression can be written

$$
i_{2}(t)=I_{\mathrm{dc}}+\hat{i}_{2 \omega_{\mathrm{o}}} \sin \left(2 \omega_{\mathrm{o}} t+\varphi_{\mathrm{o}}\right)+\hat{i}_{2 \omega_{\mathrm{i}}} \sin \left(2 \omega_{\mathrm{i}} t+\varphi_{\mathrm{i}}\right)
$$

where $\hat{i}_{2 \omega_{\mathrm{o}}}$ and $\hat{i}_{2 \omega_{\mathrm{i}}}$ are the amplitudes of the AC components of the current at the frequencies $2 \omega_{\mathrm{o}}$ and $2 \omega_{\mathrm{i}}$, respectively.

The submodule input current $i_{\mathrm{i}}(t)$ can now be recalculated. It is formed as a modulation of the transformer pulse frequency and $i_{2}(t)$ as follows

$$
\begin{aligned}
i_{i}(t)= & i_{2}(t) \times \sin \left(\omega_{\mathrm{i}} t\right) \\
= & I_{\mathrm{dc}} \sin \left(\omega_{\mathrm{i}} t\right)+ \\
& \frac{\hat{i}_{2 \omega_{\mathrm{o}}}}{2}\left[\cos \left(\left(2 \omega_{\mathrm{o}}-\omega_{\mathrm{i}}\right) t+\varphi_{\mathrm{o}}\right)-\cos \left(\left(2 \omega_{\mathrm{o}}+\omega_{\mathrm{i}}\right) t+\varphi_{\mathrm{o}}\right)\right]+ \\
& \frac{\hat{i}_{2 \omega_{\mathrm{i}}}}{2}\left[\cos \left(\omega_{\mathrm{i}} t+\varphi_{\mathrm{i}}\right)-\cos \left(3 \omega_{\mathrm{i}} t+\varphi_{\mathrm{i}}\right)\right]
\end{aligned}
$$

Consequently, there are components at the input frequency (transformer pulse frequency), around the input frequency $\left(\omega_{i} \pm 2 \omega_{0}\right)$, and at triple the input frequency. One notable issue is that there are two components at the input frequency with different phases and amplitudes. The first of these components has an amplitude of $I_{\mathrm{dc}}$ and is in phase with the transformer voltage. The second component at the transformer pulse frequency leads the dominant component by $\frac{\pi}{2}$ if the input power factor is 1 , i.e. $\varphi_{i}=0$. Thus, when the dominant component is close to zero $\left(\omega_{\mathrm{i}} t \approx 0, \pi\right.$ or $\left.2 \pi\right)$, the second component dominates.

Next, a simulation is performed to investigate the frequency spectra of the DC link voltage and the transformer winding current on the load side. The simulation is performed for a four-belt system. The motor frequency is $44 \mathrm{~Hz}$, and nominal external torque is applied. The apparent switching frequency of the load-side CHB is $8.0 \mathrm{kHz}$ making the switching frequency of one cell equal to $2.0 \mathrm{kHz}$. The transformer operating frequency is $18.0 \mathrm{kHz}$. A $1 \mu$ s sample time is used, and a Fast Fourier Transform (FFT) is calculated from the data points from the one-second time span. Thus, the number of samples is one million, and the frequency resolution is $1 \mathrm{~Hz}$. The average value of the DC link voltage is subtracted from the signal for which the FFT is calculated. The spectrum estimates are shown in Figures $3.42-3.45$.

The dominant components of the frequency spectrum were accurately predicted by the simplified analysis: the DC link voltage includes the $2 f_{\text {mot }}=88 \mathrm{~Hz}$ component, and the winding current includes the components $f_{\mathrm{sw} \text {,trafo }}=18 \mathrm{kHz}, f_{\mathrm{sw} \text {,trafo }} \pm 2 f_{\text {mot }}=18 \pm 0.088 \mathrm{kHz}$, and $3 f_{\mathrm{sw}, \text { trafo }}=54 \mathrm{kHz}$. The DC link voltage includes the predicted $2 f_{\mathrm{sw}, \text { trafo }}=36 \mathrm{kHz}$, but its magnitude is very low as a result of the low impedance of the DC link capacitor at high frequencies. The switching behavior of the output cell has quite a dramatic effect on both of the frequency spectrum estimates. As the cell switching frequency is $2 \mathrm{kHz}$, the attenuation produced by the DC link capacitor is not sufficient to suppress this component. Therefore, this 


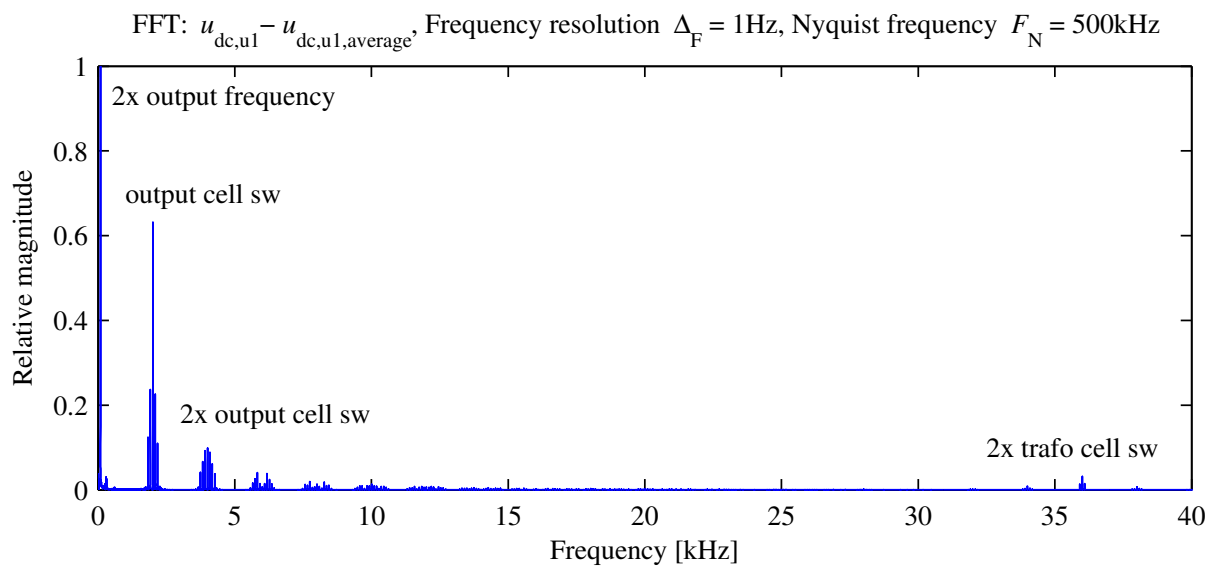

Figure 3.42. Frequency spectrum estimate of the submodule DC link voltage.

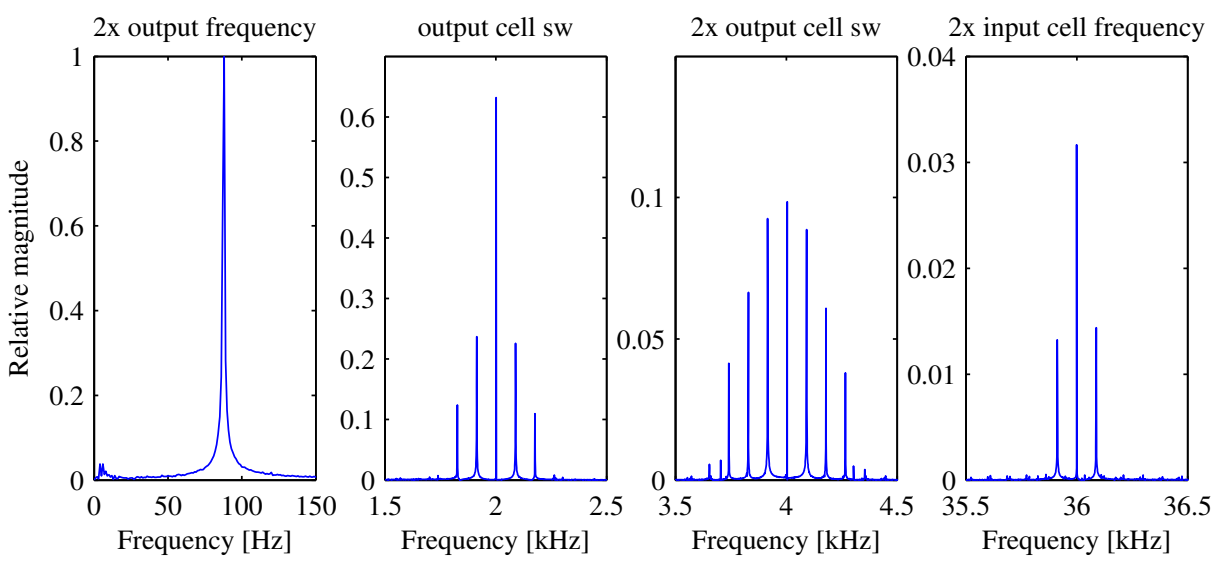

Figure 3.43. Submodule DC link voltage spectrum estimate focused on the dominant peaks.

component is translated into the winding currents similarly to the double output frequency component.

The switching harmonics of the cells were not taken into account in the preliminary analysis, but the frequencies and their harmonics generate a set of additional components of their own to the spectrum of DC link voltages, and consequently, to the actual winding current. These components together with the magnetizing current should be added to (3.71) for a complete description of the current. It should be noted that the magnetizing current adds a third component at the frequency $\omega_{\mathrm{i}}$ that lags the transformer voltage by $\frac{\pi}{2}$.

When the load-side DC links are considered, the AC components are twice the transformer operating frequency and twice the motor frequency. At the grid-side submodules, the latter 
FFT: $i_{\mathrm{S} 1,1}$, Frequency resolution $\Delta_{\mathrm{F}}=1 \mathrm{~Hz}$, Nyquist frequency $F_{\mathrm{N}}=500 \mathrm{kHz}$

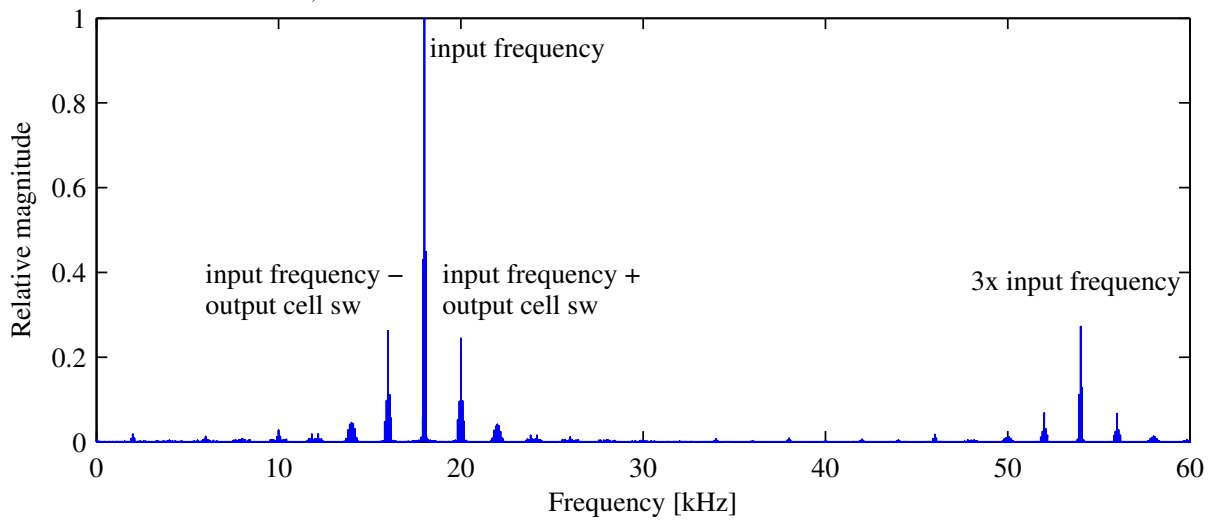

Figure 3.44. Frequency spectrum estimate of a transformer winding current.

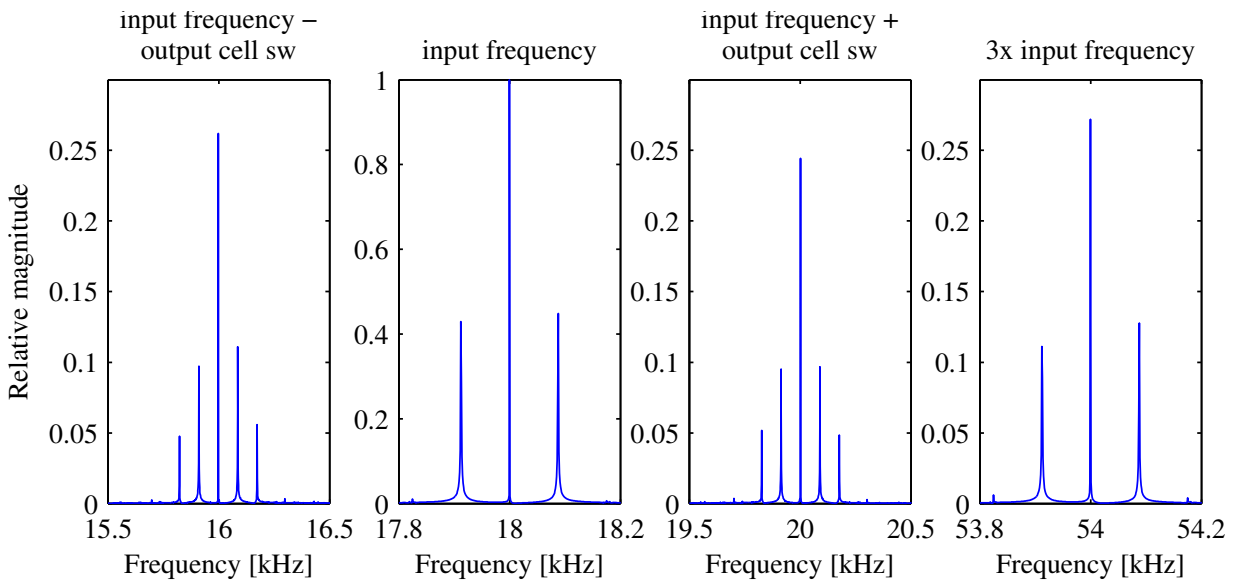

Figure 3.45. Transformer winding current spectrum estimate focused on the dominant peaks.

component is twice the grid frequency. As the energy flow through the transformer is based on the voltage difference of the feeding and receiving submodules, and energy is transferred from the grid to the motor, it is expected that the DC link voltages will also contain a frequency component that is a linear combination of the grid and motor frequencies. However, this component is not visible in the DC link voltage or the winding current; see Figures 3.43 and 3.45. The three-phase input and output of the belt explain this feature: The amplitude of this frequency component is attenuated because the feeding submodule changes when the DC link voltage difference of the original source-sink pair decreases. The twice fundamental frequency ripple is 120 degrees phase shifted between the submodules on either side of the isolation, and a new submodule will become the source when the DC link voltage of the original source goes down with the ripple voltage. 


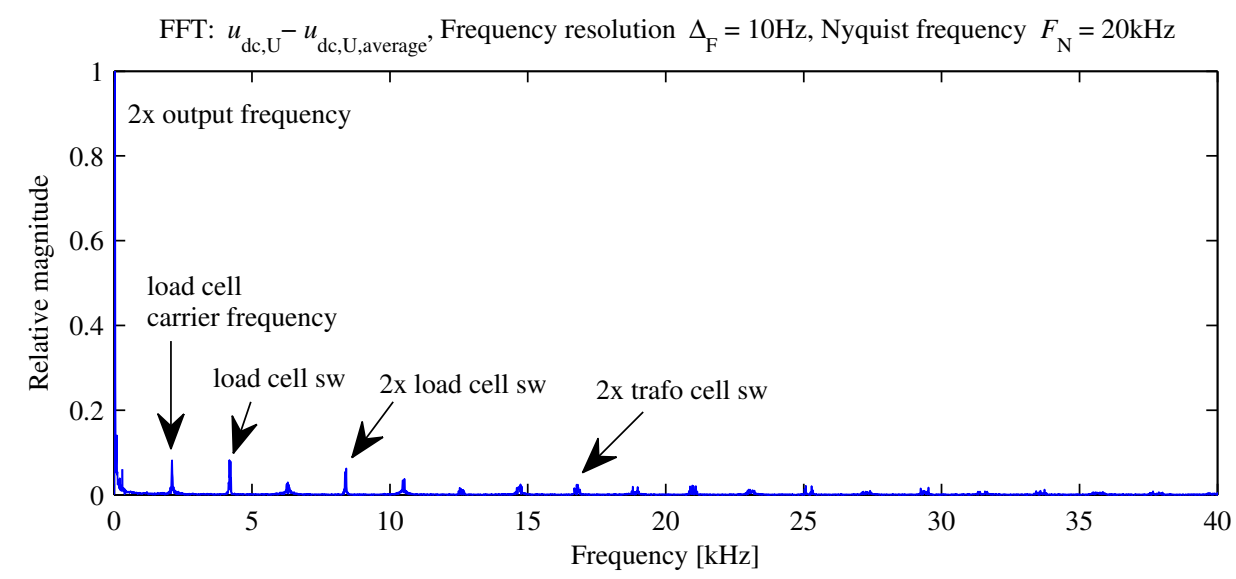

Figure 3.46. Frequency spectrum estimate of a submodule DC link voltage. The result is obtained from the experimental setup.

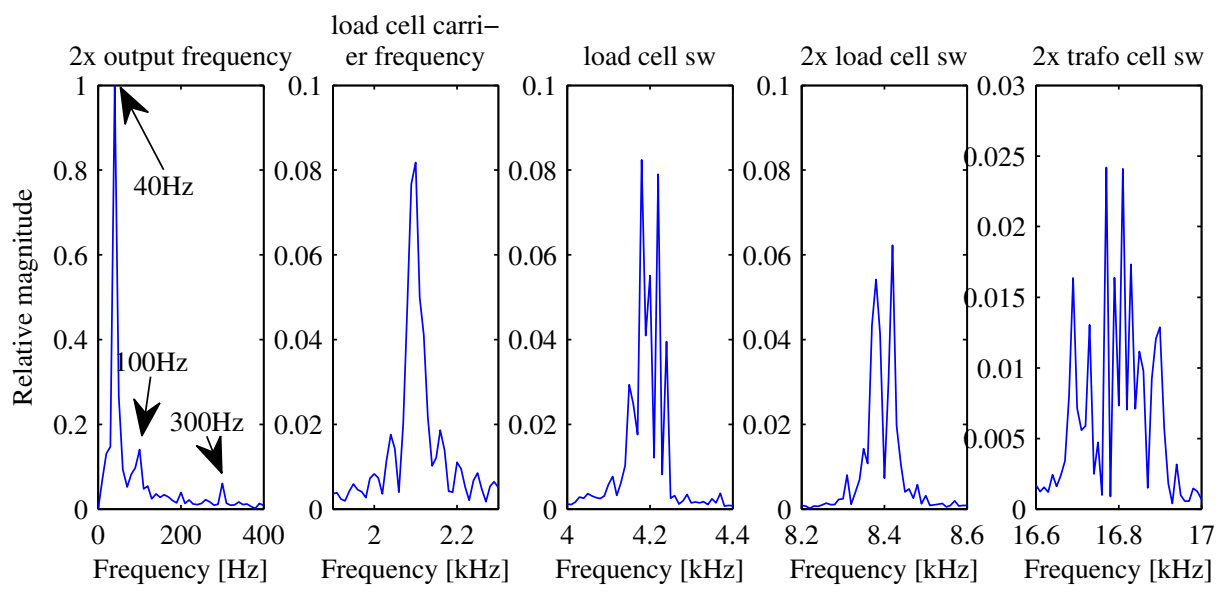

Figure 3.47. Estimate of the submodule DC link voltage spectrum focused on the dominant peaks. The result is obtained from the experimental setup.

To support the analysis given in this section, measurement results are provided in Figures 3.46-3.49. In the measurements, a low-voltage induction motor was operated with one belt. The grid voltage and frequency were $400 \mathrm{~V}$ (line-to-line) and $50 \mathrm{~Hz}$. The motor was running at $600 \mathrm{rpm}$, and the output frequency of the belt was set at $21 \mathrm{~Hz}$. The motor operating point was $P_{\mathrm{mot}} \approx 53 \mathrm{~kW}$ and the power factor $\cos \varphi_{\mathrm{mot}} \approx 0.85$. The switching frequencies of the transformer and the load-side H-bridges were $f_{\mathrm{sw} \text {,trafo }}=8.4 \mathrm{kHz}$ and $f_{\mathrm{sw} \text {,cell }}=4.2 \mathrm{kHz}$, respectively. Further details of the measurement setup are given in Section 3.7.

The spectrum estimate of a measured secondary-side DC link voltage shows the same spikes as the simulation result. The component at $8.4 \mathrm{kHz}$ equals the transformer operating fre- 
FFT: $i_{\mathrm{S} 1}$, Frequency resolution $\Delta_{\mathrm{F}}=10 \mathrm{~Hz}$, Nyquist frequency $F_{\mathrm{N}}=20 \mathrm{MHz}$

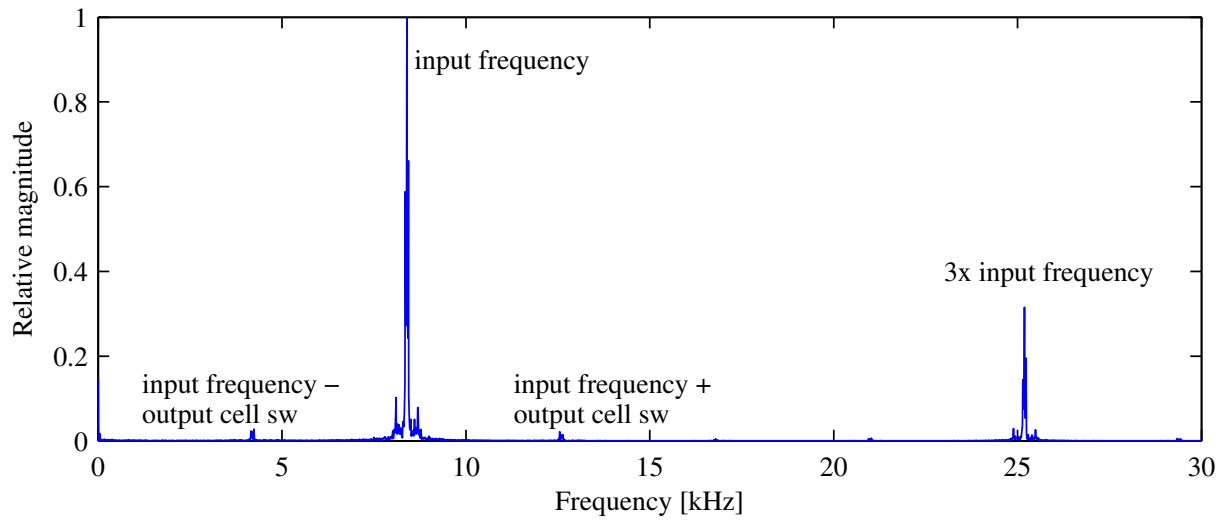

Figure 3.48. Frequency spectrum estimate of a transformer secondary-side winding current. The result is obtained from the experimental setup.
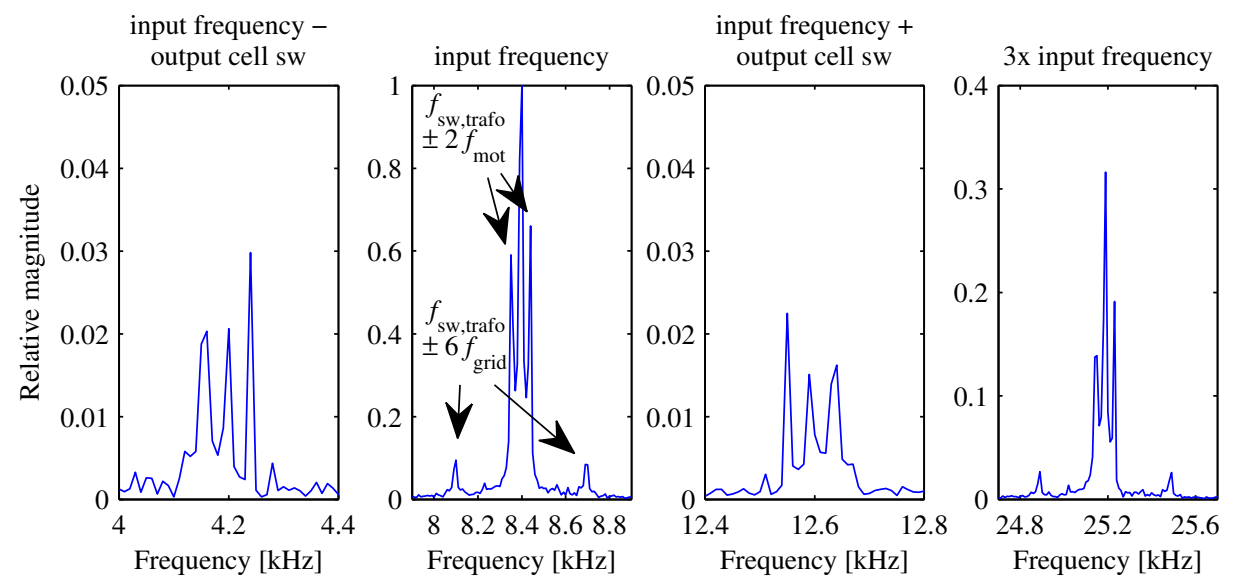

Figure 3.49. Estimate of the transformer winding current spectrum focused on the dominant peaks. The result is obtained from the experimental setup.

quency, but it is actually caused by the double output cell frequency. The relative magnitudes of the frequency spikes are different from the simulated case for three reasons: 1) a different DC link capacitance ( $1 \mathrm{mF}$ in the simulations, $10 \mathrm{mF}$ in the experimental setup), 2) a different output cell switching frequency $(2 \mathrm{kHz}$ in the simulations, $4.2 \mathrm{kHz}$ in the measurements), and 3) a different operating point of the motor. Double and six times the grid frequency are also visible in the measured DC link voltage because the impedance of the transformer link is higher in the experimental setup and the transformer is asymmetric. An additional spike at the carrier frequency of the load cell $f_{\text {car }}=0.5 f_{\text {sw.cell }}=2.1 \mathrm{kHz}$ is detected with the measurement setup. The actual mechanism that generates this additional component has not been identified, but it is anticipated that the reverse-recovery current of the antiparallel diodes may 
be the reason.

The spectrum of the transformer current follows the principles delineated in the analysis. The dominant components are the transformer operating frequency, its third harmonic, and the corresponding sidebands. The $\pm 6 f_{\text {grid }}= \pm 300 \mathrm{~Hz}$ sidebands are visible in the spectrum as expected since that component was also visible in the measured DC link voltage. In the simulation result shown in Figure 3.45, the output cell switching frequency was clearly visible in the transformer current spectrum because the voltage ripple was relatively large at that frequency ( $>60 \%$ of the ripple at twice the output frequency). Therefore, it is expected that the $2.1 \mathrm{kHz}$ spike seen in the measured DC link would appear as sidebands around the transformer operating frequency in the spectrum of the measured transformer current. However, this is not the case, which raises a question about the reliability of the DC link voltage measurement. It is noted that the spike at the output cell switching frequency $(4.2 \mathrm{kHz})$ in the DC link voltage is as high as the spike at $2.1 \mathrm{kHz}$, and the sidebands $8.4 \pm 4.2 \mathrm{kHz}$ are present in the current spectrum.

\subsection{Experimental setup}

The experimental setup of the MDC is presented schematically in Figure 3.50, and a photograph depicts the construction in Figure 3.51. The main elements of the setup are nine commercial three-phase drive inverters, a specifically designed medium-frequency six-winding transformer, a dSpace controller, a computer, a communication master device, and control cards for each inverter. On the grid side, the inverters include a diode-bridge rectifier, an intermediate DC link capacitor, and an IGBT output bridge. As the inverters are three-phase units, one input and one output phase are not used. The rectifiers at the grid interface are connected in delta. The load side consists of six three-phase inverter units. One submodule is formed by a back-to-back connection of two similar inverters, both of which include a DC link capacitor and an IGBT output bridge. The DC link capacitance in a secondary-side submodule is double compared with a primary-side submodule because of the back-to-back connection. The load bridges are connected in wye, and a $110 \mathrm{~kW}$ induction motor is the load. The shaft of the motor is connected to a generator, which is connected to a grid inverter. The main parameters are given in Table 3.7.

A photograph of the prototype is shown in Figure 3.51. The cabin on the left houses the three inverters used on the grid side. The second cabin from the left contains the transformer. The three secondary-side inverters are located in the third cabin while the motorconnected inverters are inside the fourth cabin on the right. Busbars with a cross-section of $3 \mathrm{~mm} \times 30 \mathrm{~mm}$ are used to connect the inverters to the transformer and also to provide the back-to-back connection of inverter units on the secondary side.

The communication method used to synchronize the modulation of the inverter units in the experimental setup is a modified version of the method presented in (Laakkonen, 2010). Further analysis of the communication method is omitted. 


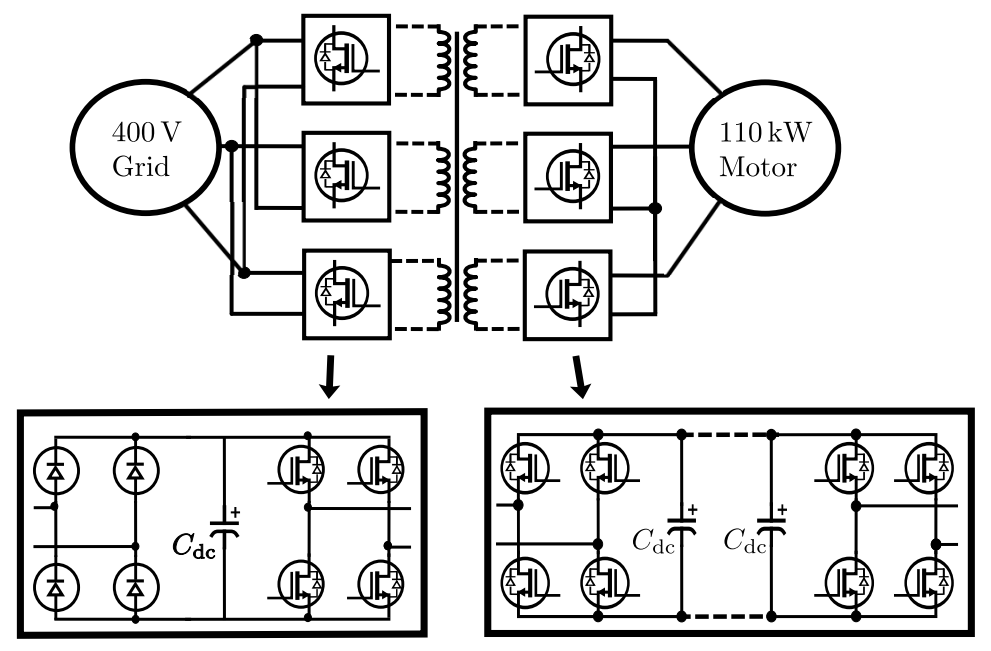

Figure 3.50. Circuit topology of the one-belt experimental setup. The connection of the inverters to the transformer and the back-to-back connection of inverters on the secondary side are made with $3 \mathrm{~mm} \times 30 \mathrm{~mm}$ busbars. The busbars are indicated by dashed lines.

The original purpose of the prototype was to 1) validate the functionality of the MDC by measuring the transformer link operation, 2) measure the efficiency, 3) verify the control principles of the transformer link, and 4) assess the dynamic behavior in different motor operating point changes. Because of setbacks in the project time schedule, poor mechanical design of the prototype, a lack of active grid bridges, and a lack of a proper motor controller, the prototype was never used in full to meet all of the initial purposes. Thus, only the functionality of the MDC could be verified with the experimental setup, and at the time of the writing of the doctoral dissertation, the prototype had already been disassembled.

Table 3.7. Parameters of the experimental system

\begin{tabular}{llll}
\hline Element & Symbol & Value & Unit \\
\hline Grid frequency & $f_{\text {grid }}$ & 50 & $\mathrm{~Hz}$ \\
Grid line-to-line voltage & $U_{\mathrm{LL}}$ & 400 & $\mathrm{~V}$ \\
Series-connected belts & $M$ & 1 & - \\
Switching frequency (load) & $f_{\mathrm{sw}, \mathrm{app}}$ & 4.2 & $\mathrm{kHz}$ \\
Transformer operating frequency & $f_{\mathrm{sw}, \text { trafo }}$ & 8.4 & $\mathrm{kHz}$ \\
Dead time (all bridges) & $t_{\mathrm{dead}}$ & 3.2 & $\mu \mathrm{s}$ \\
Primary-side DC link capacitance & $C_{\mathrm{dc}}^{\text {prim }}$ & 5.0 & $\mathrm{mF}$ \\
Secondary-side DC link capacitance & $C_{\mathrm{dc}}^{\mathrm{sec}}$ & 10.0 & $\mathrm{mF}$ \\
DC link voltage (no load) & $u_{\mathrm{dc}, \text { nom }}$ & 560 & $\mathrm{~V}$ \\
IGBT rated voltage & $V_{\mathrm{CES}}$ & 1200 & $\mathrm{~V}$ \\
\hline
\end{tabular}




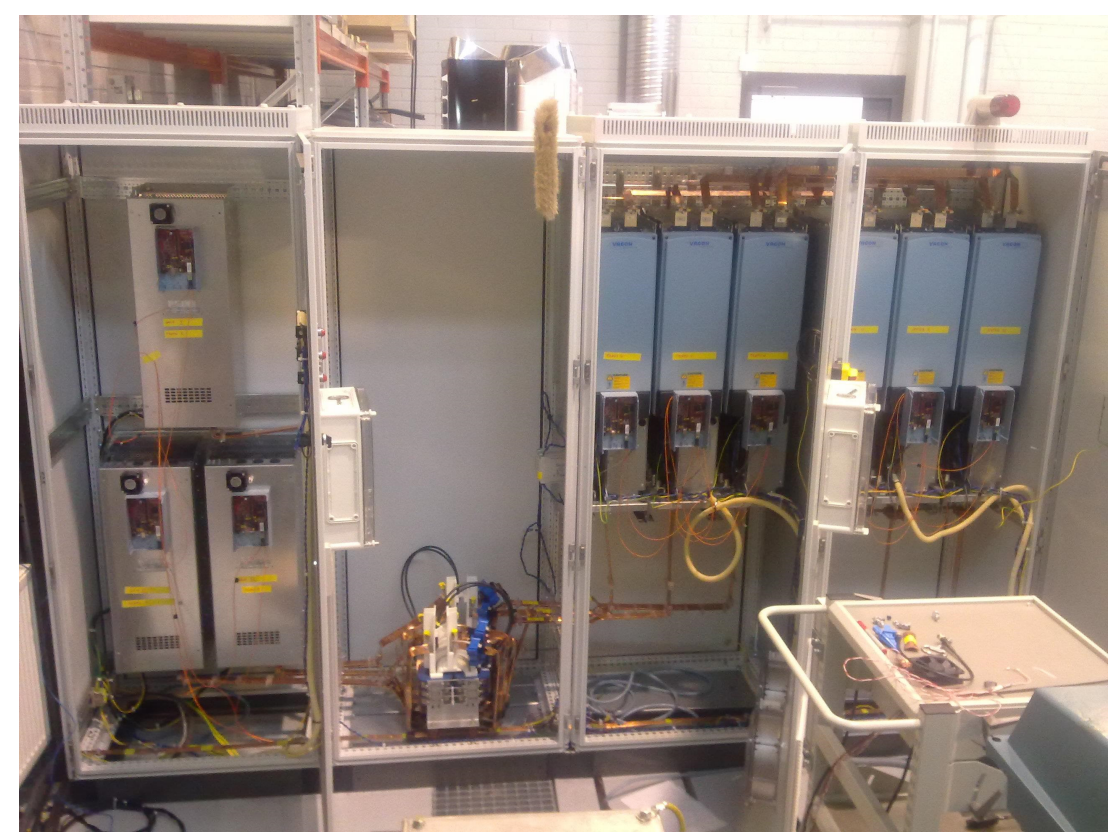

Figure 3.51. Photograph of the MDC prototype. Nine commercial inverters and a six-winding transformer are all visible. The control cards (color: red) are mounted on the front panel in each inverter. The communication of the master controller (not shown) and the inverter control cards is performed with optic fibers.

\subsubsection{Measurement results}

The functionality of the MDC topology is assessed by a series of measurements. Contrary to the simulations, the output voltage and frequency of the MDC were not controlled by an FOC. Rather, the output voltage and frequency were manually commanded from the dSpace humanmachine interface making it impossible to validate the functionality in dynamic situations. Therefore, only the steady-state performance can be assessed by the measurements.

In the measurement setup, the currents were measured with Agilent N2780A probes with a bandwidth ranging from DC to $2 \mathrm{MHz}$. The voltages were measured with Tektronix P5210 differential probes with a bandwidth from DC to 50 MHz. Two Agilent DSO6104A oscilloscopes were used, one for three concurrent current measurements and the other for three concurrent voltage measurements. The trigger signal for both of the oscilloscopes was generated at the dSpace and synchronized to the motor fundamental cycle.

Each operating point was measured in multiple steps. First, the current and voltage probes were placed at the motor terminals, and synchronized waveforms were captured. Second, the system was shut down, the probes were moved to the transformer secondary interface, and the system was restarted and driven to the desired operating point. Then, again, synchronized waveforms were captured. This procedure was continued until the currents and voltages from 
each H-bridge and the grid were obtained. Finally, the primary and secondary-side DC link voltages were measured by a similar procedure.

The measurement procedure explained above ensures that the measured current and voltage of a certain H-bridge output are synchronized. Further, the three voltages and currents obtained from one measurement are synchronized to the same instant of the motor fundamental cycle. Again, the measurements of the grid, transformer, motor, and DC voltages are synchronized to the motor fundamental cycle making a comparative analysis possible. However, the waveforms of the grid interface and the transformer primary side are not synchronized because the motor and grid waveforms are not in phase.

The steady-state performance of the prototype in two different operating points is shown in Figure 3.52. The first figure comprises the AC currents of the system. In the left-hand figure set, the motor was controlled to run at $600 \mathrm{rpm}$ by the load drive. The output frequency of the MDC was set to $21 \mathrm{~Hz}$, and the active power was approximately $53 \mathrm{~kW}$. The power was calculated from the measured motor currents and voltages. In the right-hand figure set, the motor was running at $1500 \mathrm{rpm}$ with the output frequency of the MDC set at $52.5 \mathrm{~Hz}$, and the active power of the motor was $76 \mathrm{~kW}$. Observations from the measured currents can be summarized as follows:

1. The diode rectifiers used in the grid interface cause high harmonic content;

2. The primary-side currents of the transformer exhibit a fluctuation of the envelope waveform at twice the grid frequency;

3. The secondary-side currents of the transformer exhibit a fluctuation of the envelope waveform at twice the load frequency, but also the twice grid frequency fluctuation is visible;

4. On top of the fundamentally sinusoidal motor currents, a small switching harmonic ripple is present. The raw measurement data of motor currents were filtered by a first-order lowpass filter $(-3 \mathrm{~dB}$ at $84 \mathrm{kHz})$ to filter out the high-frequency noise (the same filter was used for grid currents also);

5. The proportional difference in the currents between the two operating points is approximately the same in every current.

Considering the first remark of the above list, the total harmonic distortion of grid currents in the case of $76 \mathrm{~kW}$ active power was $34.6 \%$ with 50 harmonics included in the calculation. Remarks 2 and 3 are in accordance with the analysis in Section 3.6.2. Remark 5 is further analyzed in the following. Comparisons of the RMS and peak values of currents in different parts are shown in Tables 3.8 and 3.9. Two fundamental-frequency cycles of the grid and the motor were considered in the calculations of RMS values: all RMS values for the grid currents and the primary-side winding currents are calculated from a $40 \mathrm{~ms}$ time period whereas the RMS values for the secondary-side winding currents and the motor currents are calculated from a period of $95.2 \mathrm{~ms}$ when $f_{\text {out }}=21 \mathrm{~Hz}$ and $38.1 \mathrm{~ms}$ when $f_{\text {out }}=52.5 \mathrm{~Hz}$.

The power in the second operating point is $43 \%$ higher than in the first operating point. The change in the RMS currents is, on average, $34 \%$ in the grid, $39 \%$ in the primary windings, $52 \%$ in the secondary, and $28 \%$ in the motor. The change is highest on the secondary side, which can be explained by the lower power factor of the motor in the operating point with 

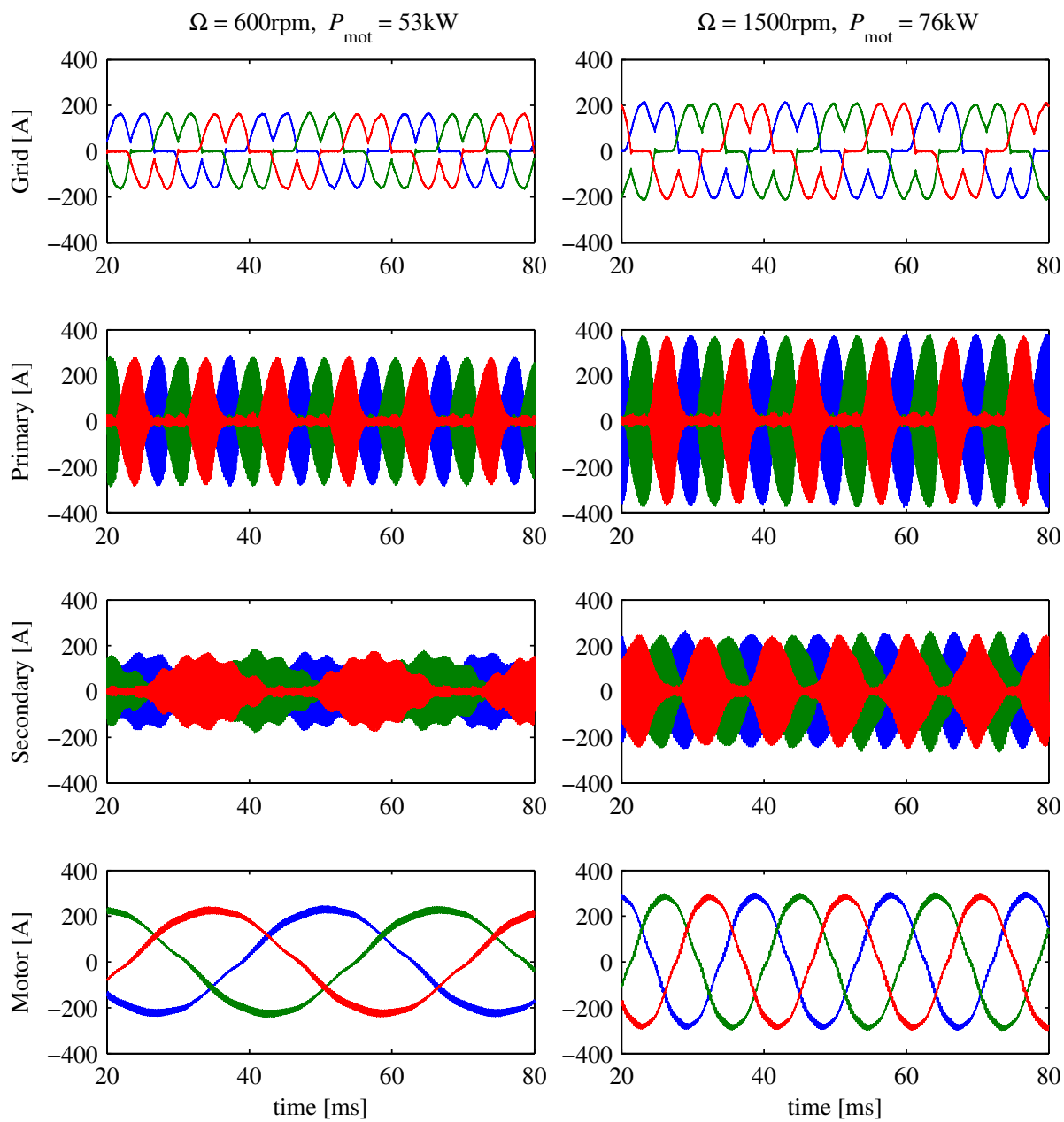

Figure 3.52. Currents of the MDC experimental system in two operating points of the motor. Colors: blue-phase A or U, green-phase B or V, red-phase C or W.

a higher active power. Indeed, the power factor is decreased from 0.83 to 0.61 when the operating point is changed. The reason for this kind of behavior of the motor voltage and current is a result of the manual tuning of the frequency and amplitude of the inverter output voltage.

The voltages of the prototype system in the second operating point are shown in Figure 3.53. The following remarks are made on the voltages:

1. The grid voltages are sinusoidal with an amplitude of $325 \mathrm{~V}$;

2. The raw data of all DC link voltages were filtered by a first-order lowpass filter $(-3 \mathrm{~dB}$ at $50 \mathrm{kHz}$ ) to get rid of the high-frequency noise, the amplitude of which was at the same level as the amplitude of twice the fundamental voltage ripple; 

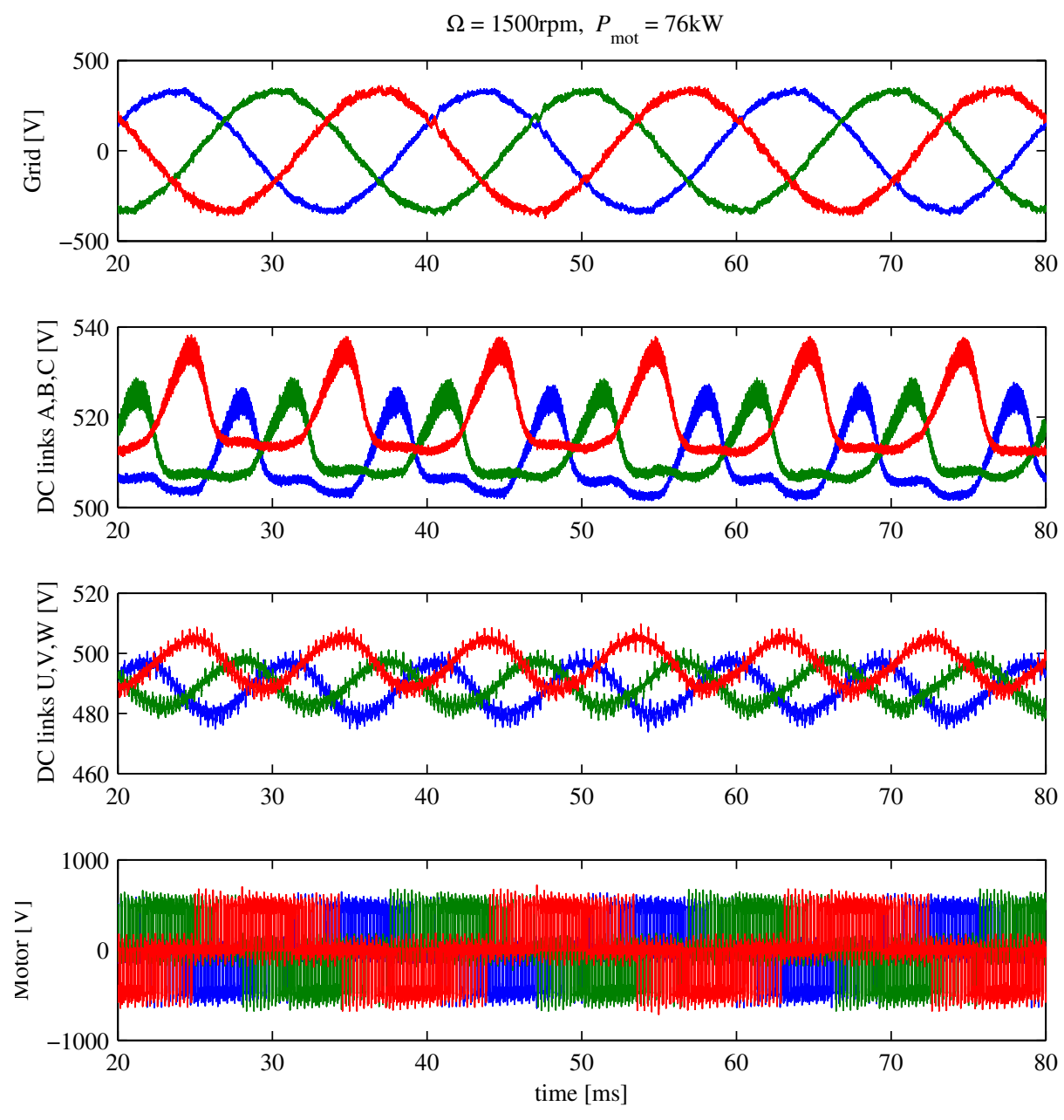

Figure 3.53. Voltages of MDC experimental system. Colors: blue-phase A or U, green-phase B or $\mathrm{V}$, red-phase $\mathrm{C}$ or $\mathrm{W}$.

3. DC link voltages in the submodules of phases $\mathrm{C}$ and $\mathrm{W}$ show a positive offset of over $10 \mathrm{~V}$ compared with the other DC links on the same side of isolation;

4. An approximately $20 \mathrm{~V}$ voltage drop occurs over the transformer.

5. Motor bridge output voltages have three levels. A high-frequency ripple is visible.

The offset seen in the DC link voltages of phases $\mathrm{C}$ and $\mathrm{W}$ may be due to improper calibration of the differential voltage probes. It is noted that the very same probe was used to obtain these two measurements. In addition to the measurement setup described above, all inverter units sent internal measurement data to the central controller at dSpace. The DC link voltages obtained by this means showed an offset of less than one volt between the voltages of different DC links. Therefore, the voltage probe calibration is blamed for the offset seen in Figure 3.53. 


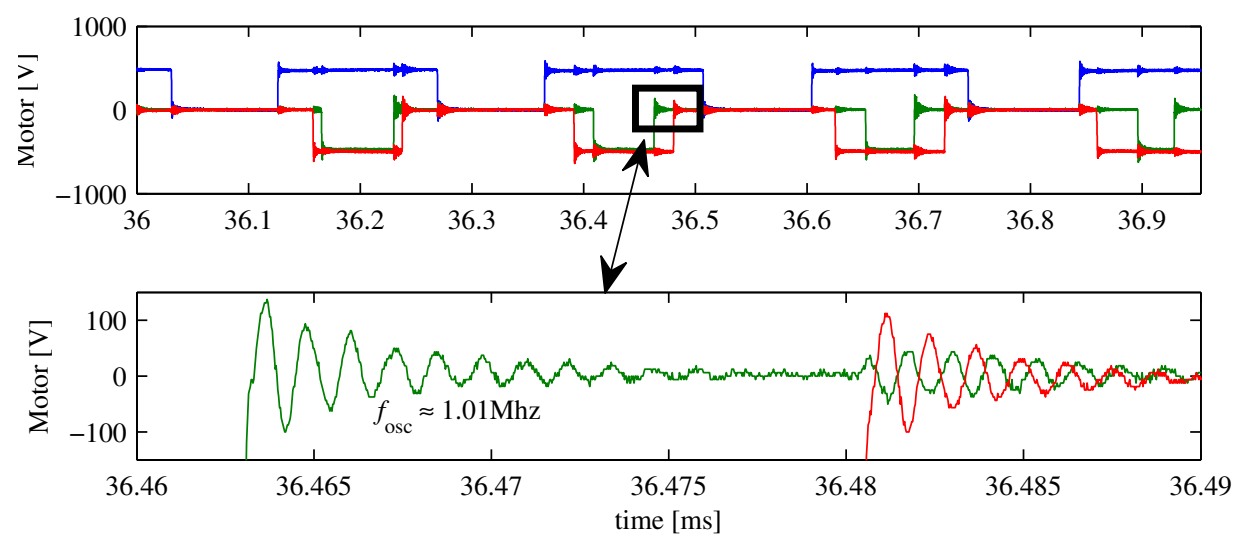

Figure 3.54. Motor bridge output voltages of the MDC experimental system. Oscillation at a very high frequency is detected. Switching in any phase excites oscillation in every phase.

Table 3.8. RMS values of the AC currents in different parts of the MDC prototype. The values are in amperes. The percentage values describe the average increase in the corresponding current between the two operating points.

\begin{tabular}{llllllll} 
Operating point & $\Omega=$ & 600 & $\mathrm{rpm}$ & $\Omega=$ & 1500 & $\mathrm{rpm}$ & \\
\hline Phase & $\mathrm{A} / \mathrm{U}$ & $\mathrm{B} / \mathrm{V}$ & $\mathrm{C} / \mathrm{W}$ & $\mathrm{A} / \mathrm{U}$ & $\mathrm{B} / \mathrm{V}$ & $\mathrm{C} / \mathrm{W}$ & \\
\hline Grid currents & 99 & 99 & 99 & 134 & 133 & 132 & $134 \%$ \\
Primary windings & 74 & 75 & 73 & 103 & 105 & 100 & $139 \%$ \\
Secondary windings & 53 & 53 & 52 & 80 & 82 & 78 & $152 \%$ \\
Motor currents & 161 & 161 & 160 & 206 & 206 & 204 & $128 \%$ \\
\hline
\end{tabular}

The high-frequency ripple of the motor-bridge output voltages is examined further in Figure 3.54. The upper plot shows a time period of four modulation cycles. It is seen that each switching transition excites a high-frequency oscillation in each output phase. The lower plot shows a zoom-in of two consecutive switching instants. The oscillation frequency is determined from the time difference of two peaks in the oscillations: $f_{\text {osc }}=1.01 \mathrm{MHz}$. The physical mechanism that generates this oscillation is unknown, but future research is expected to reveal if this behavior is a characteristic of the MDC. The oscillation has a peak value of approximately $150 \mathrm{~V}$, which is about $30 \%$ of the DC link voltage. 
Table 3.9. Peak values of the AC currents in different parts of the MDC prototype. The values are in amperes. The percentage values describe the average increase in the corresponding current between the two operating points.

\begin{tabular}{llllllll} 
Operating point & $\Omega=$ & 600 & $\mathrm{rpm}$ & $\Omega=$ & 1500 & $\mathrm{rpm}$ & \\
\hline Phase & $\mathrm{A} / \mathrm{U}$ & $\mathrm{B} / \mathrm{V}$ & $\mathrm{C} / \mathrm{W}$ & $\mathrm{A} / \mathrm{U}$ & $\mathrm{B} / \mathrm{V}$ & $\mathrm{C} / \mathrm{W}$ & \\
\hline Grid currents & 166 & 165 & 166 & 214 & 213 & 212 & $129 \%$ \\
Primary windings & 288 & 281 & 278 & 379 & 381 & 372 & $134 \%$ \\
Secondary windings & 181 & 172 & 172 & 257 & 263 & 250 & $147 \%$ \\
Motor currents & 245 & 241 & 240 & 305 & 302 & 299 & $125 \%$ \\
\hline
\end{tabular}




\section{Chapter 4}

\section{Feasibility study of the MDC}

This chapter aims to assess the feasibility of the MDC from the technical point of view. In order to accomplish this goal, the research is divided into two sections: The first section provides a comparison of the properties of the MDC and the existing topologies presented in Chapter 2. The component counts (passive and active) among other factors are compared in an example application.

The second section comprises various simulation studies aiming to demonstrate the performance of the MDC. The second section is divided into parts with the focus on the following: transformer voltage stress, performance in overloading conditions, input current quality, effect of an asymmetric transformer, introduction of a seventh winding, and common-mode voltage and current production. The third section summarizes the results of the chapter.

The efficiency and reliability of the converter topology are very important factors having a major impact on the actual feasibility of the topology. Although interesting and important, these aspects are left for future research mainly because of the lack of means to perform the required research. When it comes to efficiency, simulations with the tools available are not sufficient to give reliable results. On the other hand, the prototype system, described in Section 3.7, was not even close to an optimal design efficiencywise: The nine commercial inverters were initially three-phase inverters with an overrated current handling capability, making their thermal design nonoptimal for the MDC application. Moreover, the grid interface was constructed with diode bridges, which makes the operation of the prototype different from the intended operation of the MDC. For these reasons, the efficiency of the prototype system does not represent the actual efficiency of the MDC and was never measured. 


\subsection{Comparison of the MDC with the existing topologies in an example application}

In this section, the MDC is compared with other topologies in a specific application. The selected example is an actual application, which was described in brief at the beginning of Chapter 2: a downhill conveyor system in a Chilean mine. This application was selected because the application requires $4 \mathrm{Q}$ operation, and detailed published material is available about the different drive and motor configurations considered for the application (Rodriguez et al., 2002a,b).

In this application, a downhill conveyor carries minerals approximately 5800 tons per hour $1.3 \mathrm{~km}$ down a mountainside on a $12.6 \mathrm{~km}$ long track. The electrical system consists of three induction motor drives, $2.5 \mathrm{MW}$ each. The motors are characterized by the data given in Table 4.1. The supply voltage in the grid is $23 \mathrm{kV}$ (line-to-line). The drive system needed for one motor only is discussed in the following.

The general requirements in a conveyor system can be listed as follows (Rodriguez et al., 2002b):

1. high availability, continuous operation must be assured;

2. high reliability, avoidance of unprogrammed interruptions;

3. smooth control of torque to suppress tension in the conveyor belt during transient operation and to reduce the wear-out rate of the rotating parts;

4. sufficient controller, which ensures equal load sharing among the different motors and provides starting and braking ramps;

5. robust drive systems, perturbations in the grid voltage must have a minimum effect on the operation of the conveyor;

6. option to change the speed of the conveyor belt;

7. low current harmonics produced to the supplying network and a high input power factor;

8. field experience

Further, the requirements specific to the downhill conveyor system are listed as:

1. continuous braking torque production capability of the drive system;

2. regenerative operation, e.g. energy produced by the reducing altitude of the mass on the conveyor belt must be transmitted to the grid;

3. controlled braking of the conveyor belt in the case of power loss of the grid

The load-commutated inverter and cycloconverter are left out of the analysis here because the LCI cannot be used to drive induction motors, and the CC is not suitable for the required supply frequency of the motor. Therefore, the comparative analysis is performed for the CSI, CHB, UNIFLEX-PM, 5LANPC, and MDC. The original research on the possible drive configurations (inverter/motor pair) comprised synchronous motors, cycloconverters, and a current source PWM inverter, but eventually, the VSI technology coupled with induction 
4.1 Comparison of the MDC with the existing topologies in an example application 111

Table 4.1. Parameters of the induction motors in the downhill conveyor system.

\begin{tabular}{lll}
\hline Nominal frequency & $f_{\mathrm{m}, \mathrm{nom}}$ & $50 \mathrm{~Hz}$ \\
Nominal voltage & $U_{\mathrm{m}, \mathrm{nom}}$ & $3000 \mathrm{~V}$ \\
Nominal power & $P_{\mathrm{mot}}$ & $2500 \mathrm{~kW}$ \\
Motor pole pairs & $p_{\mathrm{m}}$ & 3 \\
Efficiency & $\eta$ & 0.96 \\
Power factor & $\cos \varphi$ & 0.87 \\
Nominal speed & $\Omega_{\mathrm{nom}}$ & $995 \mathrm{rpm}$ \\
Moment of inertia & $\mathrm{J}$ & $360 \mathrm{kgm}^{2}$ \\
\hline
\end{tabular}

motors was found to be the best alternative. The three-level NPC-topology was selected for the original application (Rodriguez et al., 2002b).

Since the supply voltage in the application is $23 \mathrm{kV}$, each topology (including MDC) requires a step-down input transformer. Because $4 \mathrm{Q}$ operation is required, all topologies should be equipped with active bridges in the grid interface. In the following, the configurations for different topologies are presented:

CSI: At the grid interface, a twelve-pulse rectification is obtained through a series connection of two six-pulse thyristor rectifiers (line-commutated rectifiers) connected to the secondaries of the input step-down transformer. The two secondaries of the transformer are phase shifted to cancel out the 5 th and 7 th harmonics from the line current. The voltage rating for the input bridge thyristors should be approximately $3 \mathrm{kV}$. The induction motor is driven by a PWM CSI employing four gate-turn-off thyristors per output phase, where a series connection of two GTOs makes up one switch. The series connection allows the use of components with half the voltage rating compared with the case of one thyristor per switch. The GTOs are also rated for approximately $3 \mathrm{kV}$. Both rectifiers and the inverter need three output capacitors each to filter out commutation-induced voltage spikes. This is the preferred CSI design for this application presented in (Rodriguez et al., 2002b).

CHB: The design is based on a cascade connection of three cells. The DC link voltage of a cell is controlled to $1100 \mathrm{~V}$ by an AFE. A three-phase IGBT input bridge is included in each cell to meet the requirement of $4 \mathrm{Q}$ operation. An L-filter is used to filter unwanted harmonics from the grid current. All IGBTs are rated for $1700 \mathrm{~V}$. The input transformer has nine three-phase secondaries with a voltage of $1000 \mathrm{~V}$ (line-to-line).

UNIFLEX-PM: Similarly to the CHB, a cascade connection of three cells is assumed with the $1100 \mathrm{~V}$ DC link voltage. The input step-down transformer has one three-phase secondary with a $3.3 \mathrm{kV}$ voltage (line-to-line). All IGBTs are rated for $1700 \mathrm{~V}$.

5LANPC: The datasheet for the ABB ACS2000 product specifies the output voltage range as $4.0-6.9 \mathrm{kV}$ and the power range as $250-2500 \mathrm{~kW}(\mathrm{ABB}, 2012)$. Thus, the specified application is below the voltage rating of the product and, at the same time, at the upper limit of the power range. The drive reaches the $2.5 \mathrm{MW}$ power when the rated voltage is 
6.6-6.9 kV. If the input transformer is designed to produce the specified voltage but the motor rating remains at $3.0 \mathrm{kV}$, the nominal power of the motor would require approximately twice the rated current of the ACS2000. In practice, this means that the product is not perfectly suitable for the application. However, this is a limitation of the product, not the topology. Thus, the following artificial design is used in the comparison of the topologies: The input transformer output voltage is $3.3 \mathrm{kV}$. Both the AFE and the inverter include eight IGBTs per phase. The DC link voltage is $5.0 \mathrm{kV}$ with a $7.1 \%$ boost ratio, and thus, half of the IGBTs are rated for $4.5 \mathrm{kV}$ while the other half for $3.3 \mathrm{kV}$.

MDC: Even though the MDC is capable of voltage transformation, a step-down from $23 \mathrm{kV}$ to $3.0 \mathrm{kV}$ would require a nonpractical solution: 18 cascaded belts on the grid side and, on the motor side, three cascaded belts and six belts in parallel. The power through each belt would be approximately $140 \mathrm{~kW}$, which constitutes under $20 \%$ of the rated belt power $(750 \mathrm{~kW})$. Thus, the input step-down transformer is a more practical solution. Similarly to the CHB and the UNIFLEX-PM, a cascaded connection of three cells with $1100 \mathrm{~V}$ DC link voltages is used. The step-down transformer has one three-phase secondary with a voltage of $3.3 \mathrm{kV}$ (line-to-line). All IGBTs are rated for $1700 \mathrm{~V}$.

Tables 4.2 and 4.3 summarize the hardware required for the main circuit of the different topologies. It is evident from the tables that a fair comparison of the topologies is not straightforward based on the hardware alone. The component count, both passive and active, is the lowest with the current source topology. However, the dynamic performance of the CSI is not on par with the VSI topologies. The 5LANPC stands out of the voltage source topologies with the lowest component count: the number of IGBTs is half of that required for the CHB. On the other hand, all IGBTs in the CHB, UNIFLEX, and MDC are low-voltage transistors making it difficult to draw conclusions based on the component count.

When the MDC and the UNIFLEX are compared, the only difference according to the tables is the number of medium-frequency transformers. Again, the transformers are not similar in the two topologies. The size of a transformer core is dictated by the flux density, which is inversely proportional to the operating frequency with a fixed voltage. The number of windings in a transformer does not affect the flux density, and therefore, if the operating frequencies and voltages match between the topologies, the total amount of core material needed is lower in the MDC as a result of the smaller number of transformers. Another factor affecting the volume of core material is the combination of the core shape and the required winding window area if the turns ratio and the winding shapes are assumed equal. In the MDC case, the six windings require a larger window area. Thus, comparison is difficult without detailed data on the transformer constructions.

However, data on the transformer constructions are available. The transformer used in the prototype system of the UNIFLEX-PM is described in detail in (Siemaszko et al., 2009). The following table compares the key figures of the UNIFLEX and MDC prototype transformers. The operating frequency of the UNIFLEX transformer is $11 \%$, and the rated power $5 \%$ of the values of the MDC transformer. Still, the MDC transformer weighs $16 \mathrm{~kg}$ less than the UNIFLEX transformer even though the number of windings is higher. Based on this comparison, the total volume and mass of the MDC transformer would be smaller than that of the 
Table 4.2. Topology hardware setup comparison

\begin{tabular}{|c|c|c|c|}
\hline Topology & Input section & DC link & Output section \\
\hline CSI & $\begin{array}{l}\text { Phase-shifting transformer; } \\
\text { C-filter; } \\
\text { Twelve-pulse thyristor bridge }\end{array}$ & Reactor & $\begin{array}{l}\text { GTO bridge; } \\
\text { C-filter }\end{array}$ \\
\hline CHB & $\begin{array}{l}\text { Phase-shifting transformer; } \\
\text { L-filter per cell; } \\
\text { Two-level three-phase } \\
\text { IGBT bridge per cell }\end{array}$ & $\begin{array}{l}\text { Single capacitor } \\
\text { per cell }(1100 \mathrm{~V})\end{array}$ & $\begin{array}{l}9 \text { cells total; } \\
\text { 7-level CHB }\end{array}$ \\
\hline UNIFLEX & $\begin{array}{l}\text { Step-down transformer; } \\
\text { LCL filter; } \\
\text { 7-level CHB }\end{array}$ & $\begin{array}{l}\text { Single capacitor } \\
\text { per submodule } \\
(1100 \mathrm{~V})\end{array}$ & 7-level CHB \\
\hline 5LANPC & $\begin{array}{l}\text { Step-down transformer; } \\
\text { LCL filter; } \\
\text { 5-level ANPC }\end{array}$ & $\begin{array}{l}\text { Dual capacitors } \\
(2.5+2.5 \mathrm{kV})\end{array}$ & $\begin{array}{l}\text { 5-level } \\
\text { ANPC }\end{array}$ \\
\hline MDC & $\begin{array}{l}\text { Step-down transformer; } \\
\text { LCL filter; } \\
\text { 7-level CHB }\end{array}$ & $\begin{array}{l}\text { Single capacitor } \\
\text { per submodule } \\
(1100 \mathrm{~V})\end{array}$ & 7-level CHB \\
\hline
\end{tabular}

Table 4.3. Topology component count comparison

\begin{tabular}{ccccc}
\hline Topology & Transformers & Capacitors & Inductors & Transistors \\
\hline CSI & $1(\mathrm{LF})$ & $6(\mathrm{AC})$ & $1(\mathrm{DC})$ & $\begin{array}{l}12 \text { Thyristors; } \\
12 \text { GTOs }\end{array}$ \\
\hline CHB & $1(\mathrm{LF})$ & $9(\mathrm{DC})$ & $9(\mathrm{AC})$ & 90 IGBTs; \\
\hline UNIFLEX & $\begin{array}{l}1(\mathrm{LF}) ; \\
9(\mathrm{MF})\end{array}$ & $\begin{array}{l}18(\mathrm{DC}) ; \\
3(\mathrm{AC})\end{array}$ & $6(\mathrm{AC})$ & 144 IGBTs; \\
\hline 5LANPC & $1(\mathrm{LF})$ & $\begin{array}{l}2(\mathrm{DC}) ; \\
3(\mathrm{AC})\end{array}$ & $6(\mathrm{AC})$ & 48 IGBTs; \\
\hline MDC & $1(\mathrm{LF}) ;$ & $\begin{array}{l}18(\mathrm{DC}) ; \\
3(\mathrm{AC})\end{array}$ & $6(\mathrm{AC})$ & 144 IGBTs; \\
\hline
\end{tabular}

UNIFLEX transformer, but this is not a fair comparison because of unequal power ratings of the two transformers. The power rating of the UNIFLEX transformer is insufficient for the application considered in this section.

Because the component count alone cannot be used to determine which of the topologies to use, the two specification lists provided at the beginning of this section are referenced. 
Table 4.4. Key figures of the UNIFLEX and MDC prototype transformers.

\begin{tabular}{ccc}
\hline Parameter & UNIFLEX & MDC \\
\hline Rated power & $36.3 \mathrm{kVA}$ & $760 \mathrm{kVA}$ \\
Operating frequency & $2.0 \mathrm{kHz}$ & $18.0 \mathrm{kHz}$ \\
Weight (core+copper) & $53 \mathrm{~kg}$ & $37.3 \mathrm{~kg}$ \\
\hline
\end{tabular}

Each topology is capable of smooth torque control, 4Q operation, varying the speed of the conveyor belt, and providing continuous braking torque. Then, the problem comes down to availability, reliability, field experience, operation during a power loss of the grid, and current harmonics produced to the grid. Interestingly, the efficiency of the converter is absent from the requirement specification lists.

It is difficult to assess the availability and reliability of converter topologies that do no exist in the real world, such as the MDC and the UNIFLEX-PM. On the other hand, an artificial design for the 5LANPC was used, and its availability and reliability are also not available. Thus, these aspects are not discussed further in the comparison.

The CSI, CHB, and 5LANPC are found in actual applications, and therefore, there is field experience available of those topologies. This aspect increases the apparent reliability of the converter, but is not a quantitative measure. Then, only the operation during disturbances in the grid and the produced current harmonics remain as viable measures on which the decision of the topology can be based.

When a power loss of the grid occurs, the energy produced by the potential energy of the mass on the conveyor belt is both an asset and a nuisance: On the one hand, the energy can be used to maintain the voltage inside the converter, and on the other hand, a certain amount of braking torque is required so that the speed of the conveyor belt does not increase. The excessive braking energy has to be dissipated in a resistive load. The CSI does not allow the use of the energy stored in the DC link in the case of a power loss of the grid. Thus, the CSI is not a viable solution for the application (Rodriguez et al., 2002b).

None of the voltage source topologies have integrated brake choppers or brake resistors. Addition of such a device is therefore a necessity for each topology to make them viable for the present application. In the 5LANPC, only one brake chopper is required since there is only one DC link. The CHB, MDC, and UNIFLEX-PM need distributed brake choppers. In the case of the $\mathrm{CHB}$, an intuitive solution is to add a brake chopper and a resistor in each cell. Similarly, the UNIFLEX-PM requires a brake chopper in each motor-side submodule. Likewise, this is an intuitive solution for the MDC, but there is also another solution available: Inside a belt, the transformer can be equipped with an additional seventh winding, where the brake chopper and the resistor can be placed. The number of brake choppers required in the MDC is three whereas the CHB and the UNIFLEX-PM need nine. The idea of the seventh winding in the MDC transformer is further discussed in Section 4.2.5. 
The dissipation of excessive braking energy during a grid blackout eliminated the option of using a CSI in the application. In the case of VSI topologies, the addition of braking choppers is required in each topology, but does not give sufficient reasons to prefer one over others. The harmonics produced by the converter to the grid currents and the dynamic performance of the converters must then be used as a basis for making the decision. A simulation model for each converter topology is required to assess these aspects; nevertheless, because of the amount of work needed to accomplish the simulation study, it is omitted. Therefore, the topology that best fits the application cannot be explicitly determined.

\subsection{Performance of the MDC}

The performance of the MDC is assessed in this section. Section 4.2.1 explores the limits of the operating voltage by studying the voltage stress experienced by the transformer. The factors affecting the overloading capability of the converter are analyzed in Section 4.2.2. The harmonic content of grid currents is assessed in Section 4.2.3, and a topology-specific feature is pointed out. Because the design of a symmetric transformer may be difficult in practice, the effect of an asymmetric transformer on the system performance is studied in Section 4.2.4. In Section 4.2.5, the potential improvement in the converter performance by a hardware modification of the transformer is presented. Finally, 4.2.6 presents the converterspecific features related to the production of common-mode voltages and currents.

\subsubsection{Transformer voltage stress}

Because of the medium-frequency transformer, the MDC is a highly modular topology. However, the modularity has its limits. The blocking voltage of the transformer isolation material sets a limit on the maximum voltage of the drive. This section explores the required blocking voltage for the transformer. Simulation results are shown.

In order to perform the simulation study, artificial star points were added to both the grid and the motor side by connecting three high-value resistors $(10 \mathrm{k} \Omega)$ to the output of the grid inverter and the motor inverter to form star points. On the grid side, this star point represents the protective earth, or in short, the ground potential. The star point on the motor-side is also connected to the ground potential to simulate the grounding of the motor chassis. In reality, the connection from the motor windings to the chassis is created by stray capacitances, and therefore, it is not resistive. Using high-value resistors to form the star points provides an opportunity to measure the voltage differences over the isolation barrier.

Figure 4.1 shows the waveforms of the voltage stress experienced by the transformer in the case of three belts when the motor is supplied with a $40 \mathrm{~Hz}$ supply voltage. Four different points are measured from two belts (1 and 3):

1. voltage from one primary-side winding to another primary-side winding, measured be- 
tween windings $1+$ and 3-;

2. voltage from one secondary-side winding to another secondary-side winding, measured between windings $4+$ and 6-;

3. voltage from one primary-side winding to a secondary-side winding, measured between windings 3 - and 4+;

4. voltage from one primary-side winding to the ground (gnd), measured between winding $1+$ and gnd.

It is seen from the voltage waveforms that the transformer in belt 1 experiences a higher voltage stress than the transformer in belt 3 . In the simulation, the main circuit matches that of Figure 3.1, where the grid and the motor are connected to belt 1, and the neutral point connection of the CHB configuration on both sides is located at belt 3 . The voltage stress between two primary windings is dictated by the grid voltage, whereas on the motor side, the motor voltage determines the voltage stress. The voltage stress over the isolation barrier (from the primary to the secondary) includes both frequency components: $50 \mathrm{~Hz}$ from the grid and $40 \mathrm{~Hz}$ from the motor.

The belt connection configuration is changed such that on the grid side, the neutral point of the CHB inverter is located at belt 3 and on the motor side at belt 1 . Thus, the grid is connected to belt 1 and the motor to belt 3 . The gate signals generated by the motor inverter modulator and the order of the DC link measurement feedbacks for the modulator are changed accordingly. The target of this change is to balance the voltage stresses of the transformers in
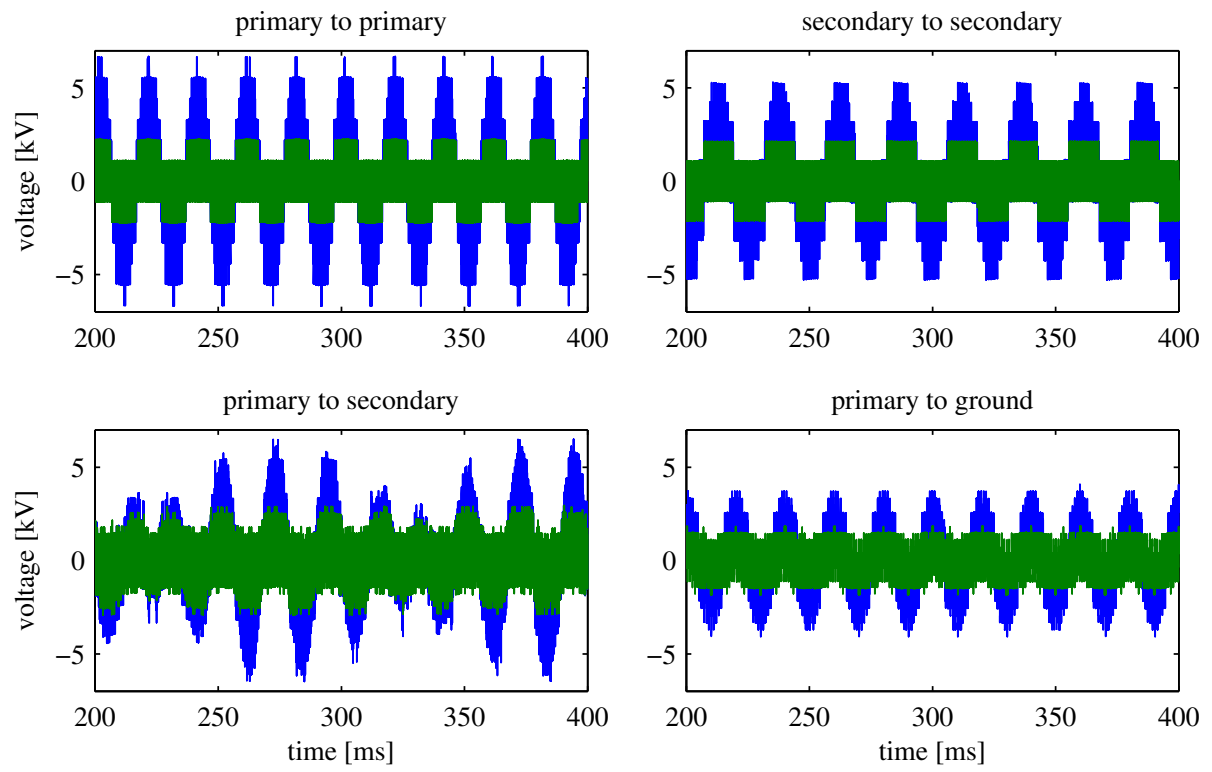

Figure 4.1. Waveform of the voltage stress of the transformer when the grid voltage is $3.3 \mathrm{kV}$ and three belts are used. The blue graphs are measured from belt 1 and the green graphs from belt 3 . 

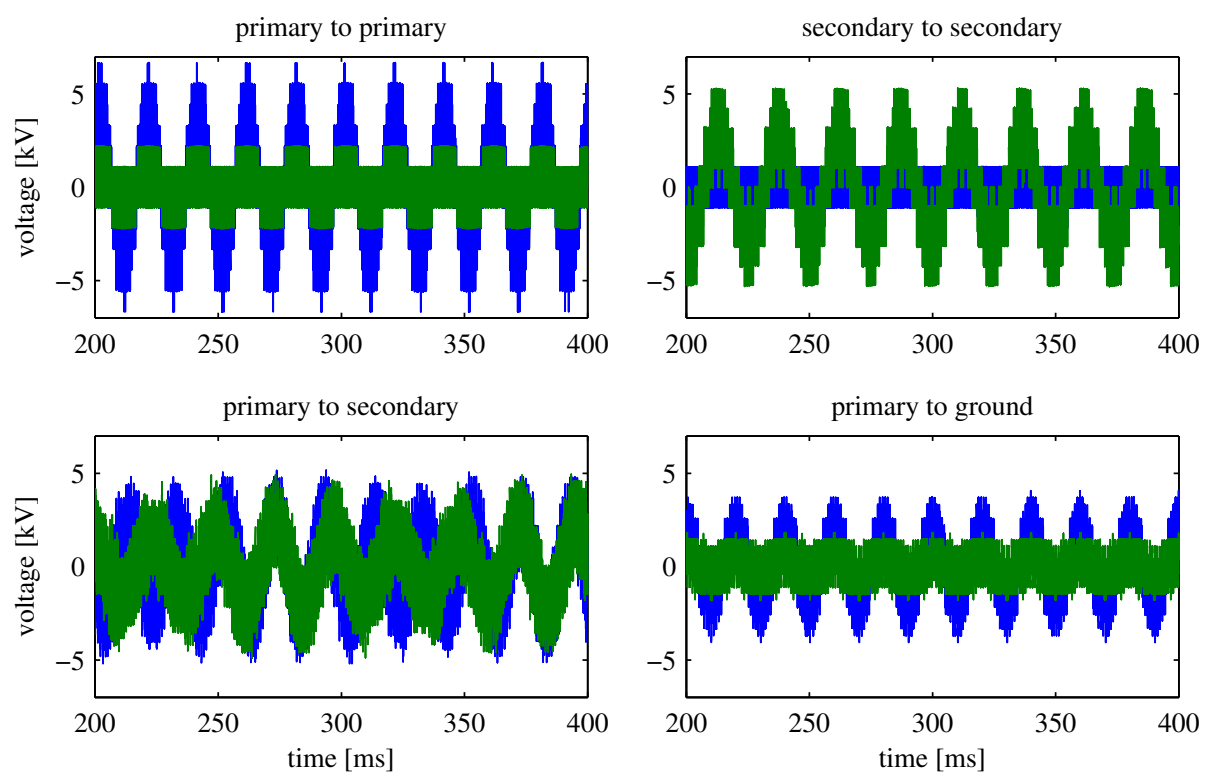

Figure 4.2. Waveform of the voltage stress of the transformer when the grid voltage is $3.3 \mathrm{kV}$, and a modified belt order is used. The blue graphs are measured from belt 1 and the green graphs from belt 3 .

different belts. The waveforms of the transformer blocking voltages in this configuration are shown in Figure 4.2.

With the motor connected to belt 3 , the voltage stress over the isolation (from the primary to the secondary) is balanced between the two belts. On the other hand, the voltage stress between the secondary windings in the transformers of belts 1 and 3 have switched places. Thus, it is evident that this configuration only balances the voltage stresses over the isolation barrier.

Further balancing of the transformer voltage stresses could be obtained by distributing the neutral point connection to multiple belts. For example in the case of three belts, one Hbridge cell from each belt is connected to the neutral point on either side of isolation. Correspondingly, one H-bridge cell from each belt is connected to one of the grid or motor phases. This kind of a configuration could, however, be a nonpractical solution as the mechanical construction of the wiring becomes complex. Additionally, the optimal configuration is not straightforward if the number of belts is a nontriplen value $(2,4,5,7, \ldots)$.

Next, the required blocking voltage in the case of different numbers of belts $(M=1 \ldots 5)$ was investigated by simulations. The neutral point of the grid inverter was in belt $M$ while the neutral point of the motor inverter was in belt 1 . Correspondingly, the grid was connected to belt 1 and the motor to belt $M$ in all simulations. The results of the simulation study are shown in Figure 4.3. The RMS and peak values of the measured voltage stresses are 

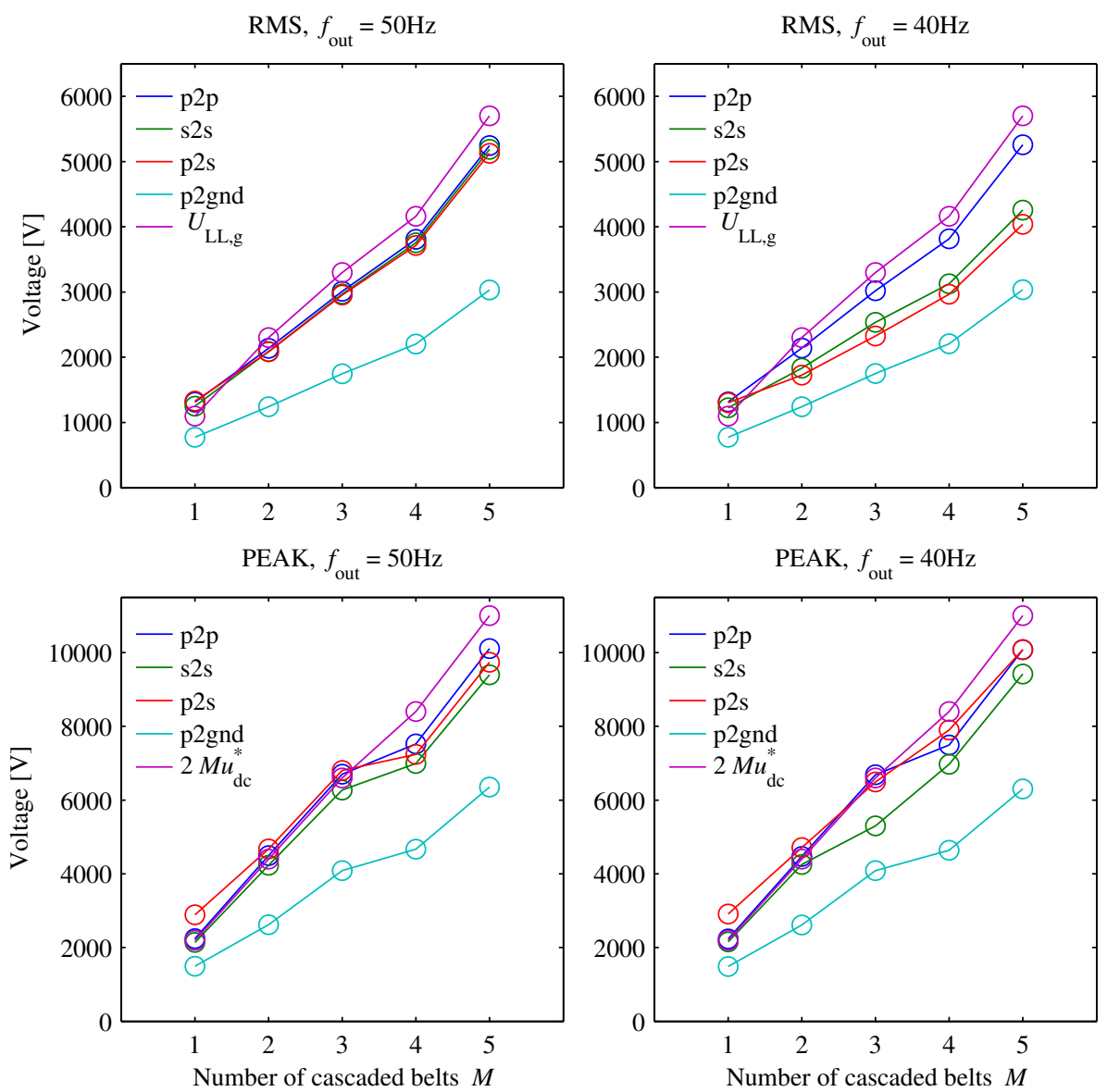

Figure 4.3. Voltage stress of the transformer under two different loading conditions as a function of number of belts. Measurements are from the transformer inside belt no. 1 .

shown in cases of $50 \mathrm{~Hz}$ and $40 \mathrm{~Hz}$ motor supply frequencies. It is evident that the grid and motor voltages play significant roles in the voltage stress production. With a lower motor frequency, also its supply voltage is lower and the voltage stresses from the primary to the secondary and between the secondaries are lower compared with the case of the $50 \mathrm{~Hz}$ motor frequency. While the RMS values of the voltage stresses correlate strongly with the grid and motor voltage values, the peak values of the voltage stresses are dictated by the DC link voltages because of the PWM operation.

The simulation study on the transformer voltage stress revealed a few points worth mentioning:

1. Location of the neutral point of the CHB configuration affects the voltage stress experi- 
enced by the belt transformers. Forming the neutral point to the first belt on the grid side and to the last belt on the motor side balances the voltage stress over the isolation barrier experienced by different transformers in the MDC;

2. The required blocking voltage capability is determined by the voltage level of the grid (dictates the RMS value), and the total DC link voltage (dictates the peak value);

3. In order to maintain the high modularity of the MDC, the transformer has to be designed according to the maximum voltage stress. Then, in applications with a lower voltage rating than the absolute maximum of the MDC, the transformer is excessively rated in terms of blocking voltage capability;

4. The transformer of the MDC prototype (see Section 3.7) had a voltage blocking capability of $13 \mathrm{kV}(\mathrm{AC})$. With this rating, the maximum number of cascaded belts with $1100 \mathrm{~V}$ DC link voltages is six, and thus, the application voltage is restricted below $7 \mathrm{kV}$ line-to-line.

Finally, it is noted that the whole construction of the belt has to withstand the same voltage stress as the transformer. In other words, the isolation between the conductors of the DC links and other conductive parts of different submodules must withstand the presented voltage stress.

\subsubsection{Overloading conditions}

The energy transfer through the transformer link is based on the voltage difference of the submodules. The stray impedance of the transformer determines the amount of current at a certain voltage difference. At the same time, the stray impedance creates a current-dependent voltage drop. This means that the higher is the power drawn by the load, the higher the voltage drop becomes. Intuitively, there is a power limit when the voltage drop over the isolation becomes too high, and problems arise. The limit set by this phenomenon is assessed by two simulations. Two series-connected belts are connected to a $2.3 \mathrm{kV}$ grid. The motor is also $2.3 \mathrm{kV}$ with a nominal power of $1.5 \mathrm{MW}$. In the first simulation, all values of the transformer stray inductance matrix are set at $L_{\sigma}=1.32 \mu \mathrm{H}$. In the second simulation, the stray inductance is divided by two to demonstrate the effect on the voltage drop and the MDC performance in an overloading situation.

The simulation starts with the nominal external torque applied to the shaft of the motor, and the speed is nominal at $1486 \mathrm{rpm}$. The external torque is incremented by steps. Each step adds another $20 \%$ of nominal torque and, after five steps, the torque is double compared with the initial value. As the motor controller aims to maintain a constant speed, the power will be doubled at the end of the simulation.

The result of the first simulation with $L_{\sigma}=1.32 \mu \mathrm{H}$ is shown in Figure 4.4. At each step, the average value of the secondary-side DC voltage decreases and the voltage ripple on both sides of the isolation increases. The average values of the DC link voltages $\left(u_{\mathrm{dc}, i j, \mathrm{filt}}\right)$ are illustrated with filtered signals shown by red graphs. The filtered signals are obtained by filtering the DC link voltages by moving average filters with $100 \mathrm{~ms}$ windows. When $160 \%$ of the nominal torque is reached (i.e., $t \approx 800 \mathrm{~ms}$ ), the voltage drop is approximately $100 \mathrm{~V}$ and the ripple on the secondary side is $200 \mathrm{~V}$. At this point, the motor supply voltage PWM 

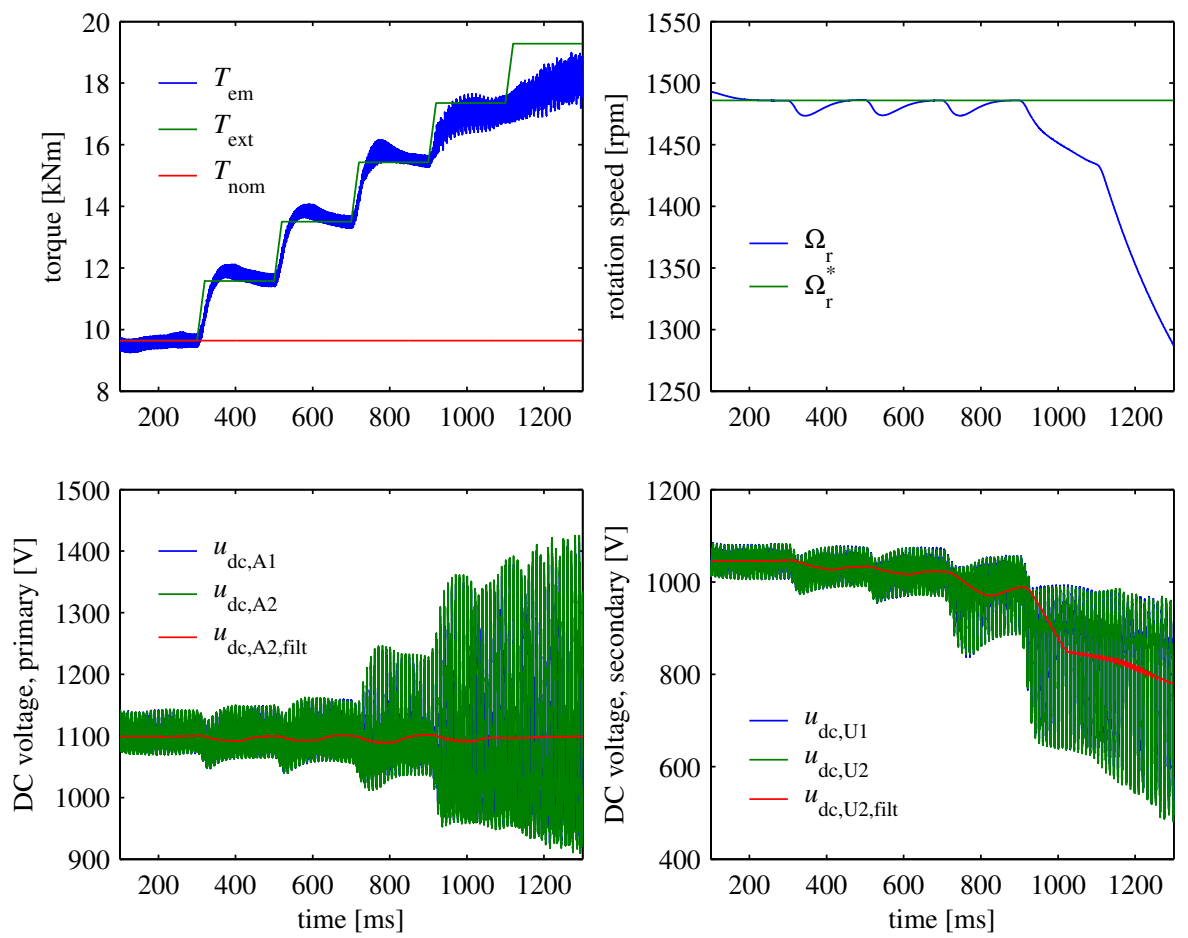

Figure 4.4. MDC performance in an overload situation. An external torque incremented by 0.2 pu until twice the nominal torque is reached. The speed cannot be maintained at the reference because of the secondary-side DC link voltage drop.

goes into overmodulation, but the converter maintains its functionality. The last two torque steps appear unbearable: The DC link voltages exhibit a ripple of more than $500 \mathrm{~V}$ when the voltage drop exceeds $200 \mathrm{~V}$. The motor controller fails to maintain the speed because of insufficient voltage available.

The result of the second simulation with the lower stray inductance of the transformer is shown in Figure 4.5. Here, the voltage drop is kept below $100 \mathrm{~V}$ even in the case of double the nominal power. The speed of the motor is maintained and the converter remains operational. This shows that special attention has to be paid to the minimization of the impedance of the transformer link when the transformer and the mechanical construction of the belt are designed.

The results of the two simulations are compared in Figure 4.6. The average voltage drops are shown. The average values are calculated as a difference of one primary-side and one secondary-side DC link voltage:

$$
u_{\mathrm{drop}, \mathrm{avg}}=u_{\mathrm{dc}, \mathrm{A} 2, \mathrm{filt}}-u_{\mathrm{dc}, \mathrm{U} 2, \text { filt }} .
$$

The average voltage drop is approximately double in the first simulation until the overmodula- 

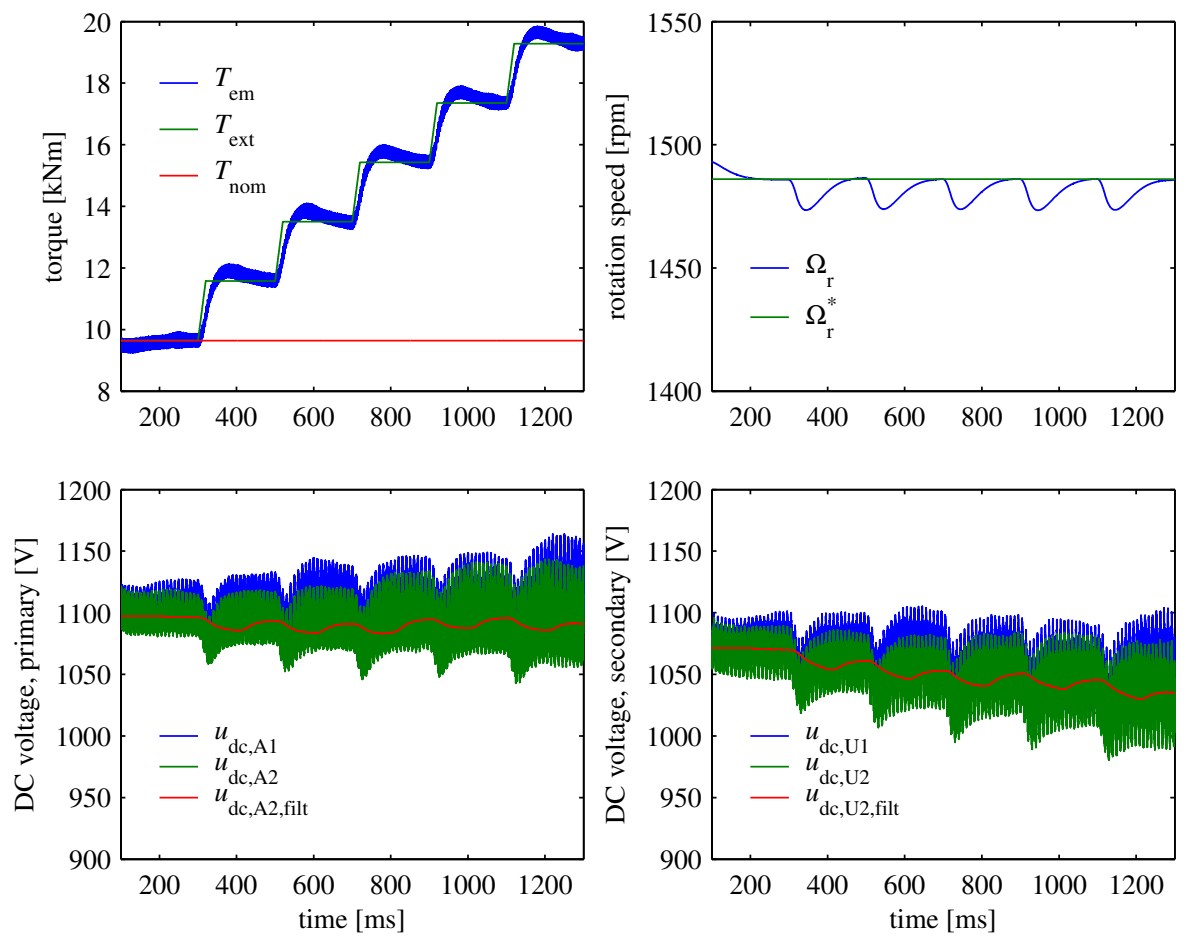

Figure 4.5. MDC performance in an overload situation with a lower stray impedance of the transformer. The external torque is incremented by consecutive steps until twice the nominal torque is reached.

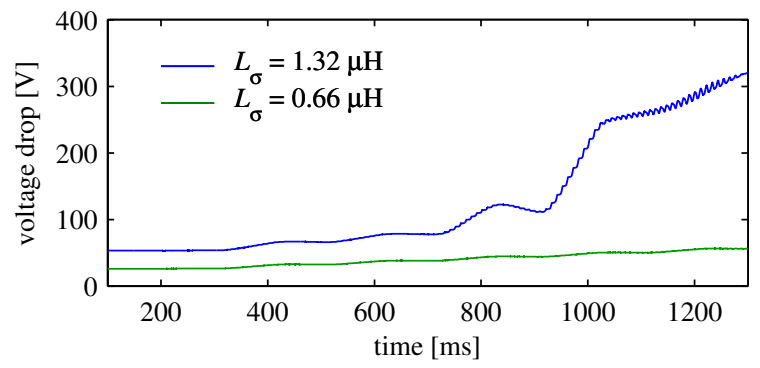

Figure 4.6. Voltage drop over the isolation in an overload situation. The external torque is incremented by consecutive steps until twice the nominal torque is reached.

tion range is entered. The system assumes nonlinear behavior, and the voltage drop increases uncontrollably.

The higher throughput power, the ripple of the DC link voltages, and the voltage drop over the transformer affect the winding currents. With $L_{\sigma}=1.32 \mu \mathrm{H}$ and the nominal power, the 
Table 4.5. Transformer winding current peak values in overloading conditions.

\begin{tabular}{ccc}
\hline External torque & $L_{\sigma}=1.32 \mu \mathrm{H}$ & $L_{\sigma}=0.66 \mu \mathrm{H}$ \\
\hline$T_{\text {ext }}=1.0 T_{\text {nom }}$ & $1210 \mathrm{~A}$ & $1210 \mathrm{~A}$ \\
$T_{\text {ext }}=1.2 T_{\text {nom }}$ & $1510 \mathrm{~A}$ & $1510 \mathrm{~A}$ \\
$T_{\text {ext }}=1.4 T_{\text {nom }}$ & $1720 \mathrm{~A}$ & $1750 \mathrm{~A}$ \\
$T_{\text {ext }}=1.6 T_{\text {nom }}$ & $2730 \mathrm{~A}$ & $2730 \mathrm{~A}$ \\
$T_{\text {ext }}=1.8 T_{\text {nom }}$ & - & $4740 \mathrm{~A}$ \\
$T_{\text {ext }}=2.0 T_{\text {nom }}$ & - & $5720 \mathrm{~A}$ \\
\hline
\end{tabular}

peak values of the winding currents are approximately $1210 \mathrm{~A}$. The following table presents the winding current peak values in different loading conditions for both simulations. Examination of the data in the table reveals three issues worth mentioning:

1. The peak value of the winding current is not impacted by the leakage impedance, because the voltage drop is smaller with lower impedance;

2. The peak value of the winding current rises with an increasing slope as the power gets higher;

3. As the power increases with a factor of two, the peak current increases with a factor of nearly five.

Since the voltage drop over the isolation is dominated by the leakage inductance, the impedance can be reduced by decreasing the operating frequency of the transformer. This method could be applied in short overloading situations. However, the method comes with certain disadvantages: First, the peak value of the transformer current will be significantly higher as a result of the longer voltage cycle (Figure 3.33). Second, the longer period time increases the applied volt-seconds (i.e., flux) leading to a saturation of the transformer if the saturation region is close to the nominal operating point. The saturation decreases the amount of magnetizing inductance leading to high peak values of winding currents, and higher core losses because of the larger hysteresis loop area.

\subsubsection{Input current quality}

An exhaustive simulation study has been performed in the course of the MDC research project. In the various simulations it has been seen that the time-domain waveforms of grid currents are distorted, and interestingly, the distortion seems to get worse as the number of cascaded belts gets higher. This section aims to assess this behavior by comparing the time-domain waveforms and frequency spectra of the simulated grid currents with different numbers of cascaded belts.

Two sets of simulations are performed for belt numbers $M=1 \ldots 5$. In the first simulation set, the apparent switching frequency of the grid inverter is $6.0 \mathrm{kHz}$ regardless of the number of belts. The second simulation uses a fixed IGBT switching frequency of $1.0 \mathrm{kHz}$ making the apparent switching frequency increase with the number of belts. The system is in the 
steady state under nominal operating conditions in all simulations. The load power is $P_{\text {load }}=$ $M \times 750 \mathrm{~kW}$. Figures 4.7 and 4.8 show the simulation results: the set of subplots on the left present the time-domain waveforms of the grid currents from a $30 \mathrm{~ms}$ time period, and the subplots on the right present the corresponding frequency spectra with the focus on the low-frequency range.

The frequency spectra are calculated by transforming the three-phase quantities into a complex vector $i_{\alpha \beta}$ in the stationary plane using the well-known transformation:

$$
\left[\begin{array}{c}
i_{\alpha} \\
i_{\beta}
\end{array}\right]=\left[\begin{array}{ccc}
\frac{2}{3} & \frac{1}{3} & \frac{1}{3} \\
0 & \frac{1}{\sqrt{3}} & -\frac{1}{\sqrt{3}}
\end{array}\right]\left[\begin{array}{c}
i_{\mathrm{A}} \\
i_{\mathrm{B}} \\
i_{\mathrm{C}}
\end{array}\right]
$$

and

$$
i_{\alpha \beta}=i_{\alpha}+j i_{\beta} .
$$

When the frequency spectrum is estimated using a Fast Fourier Transform (FFT) algorithm and the signal has complex values, the frequency spectrum estimate becomes two-sided. This means that positive and negative frequencies are separated. The sample time of the simulation is $1.0 \mu \mathrm{s}$ and the frequency spectra are estimated from $200 \mathrm{~ms}$ time spans making the length and frequency resolution of the FFT calculations equal to $200 \times 10^{3}$ samples and $5 \mathrm{~Hz}$, respectively.

When the simulated time-domain waveforms are assessed at $f_{\mathrm{sw}, \text { app }}=6.0 \mathrm{kHz}$, deterioration of the current waveform is seen. With $M=1$ and $M=2$, the waveform is seemingly sinusoidal with low harmonics. With $M>2$, distortion of the waveforms is evident. The frequency spectra give insight into the components present in each simulation. With $M=1-3$, the dominant components are the $-5^{\text {th }}$ and $7^{\text {th }}$ harmonics of the grid fundamental frequency. The negative sequence of the fundamental and the second harmonic positive sequence are present when $M=1$. The $-11^{\text {th }}$ and $13^{\text {th }}$ harmonics are amplified with $M=4$, and when $M=5$, they become the dominant harmonic components.

The components at $-f_{\text {grid }}$ and $2 f_{\text {grid }}$ result from the twice the fundamental frequency fluctuation of the DC links, while the components at $-5 f_{\text {grid }}$ and $7 f_{\text {grid }}$ result from the six times the fundamental frequency fluctuations of the DC link voltages. Actually, in the MDC, the $6 f_{\text {grid }}$ fluctuation is not present in a single submodule DC link. Rather, it is present in the virtual DC link produced by the transformer coupling of the grid-side submodules inside one belt.

It is interesting that each harmonic component gets divided into two separate peaks when $M=4$ and $M=5$. The separate peaks are $\pm 20 \mathrm{~Hz}$ from the actual harmonic component with four belts and $\pm 10 \mathrm{~Hz}$ with five belts. It is reminded here that the frequency resolution is exactly $5 \mathrm{~Hz}$, and thus, the spacing between the harmonic component and the spectrum peak may be different in reality. However, these components degrade the time-domain current waveforms with a higher number of belts. The underlying mechanism that produces these peaks is the different grid and motor supply frequencies. The motor controller adjusts the speed of the motor to $1486 \mathrm{rpm}$ in each simulation, and the output frequency is not necessarily exactly $50 \mathrm{~Hz}$. 

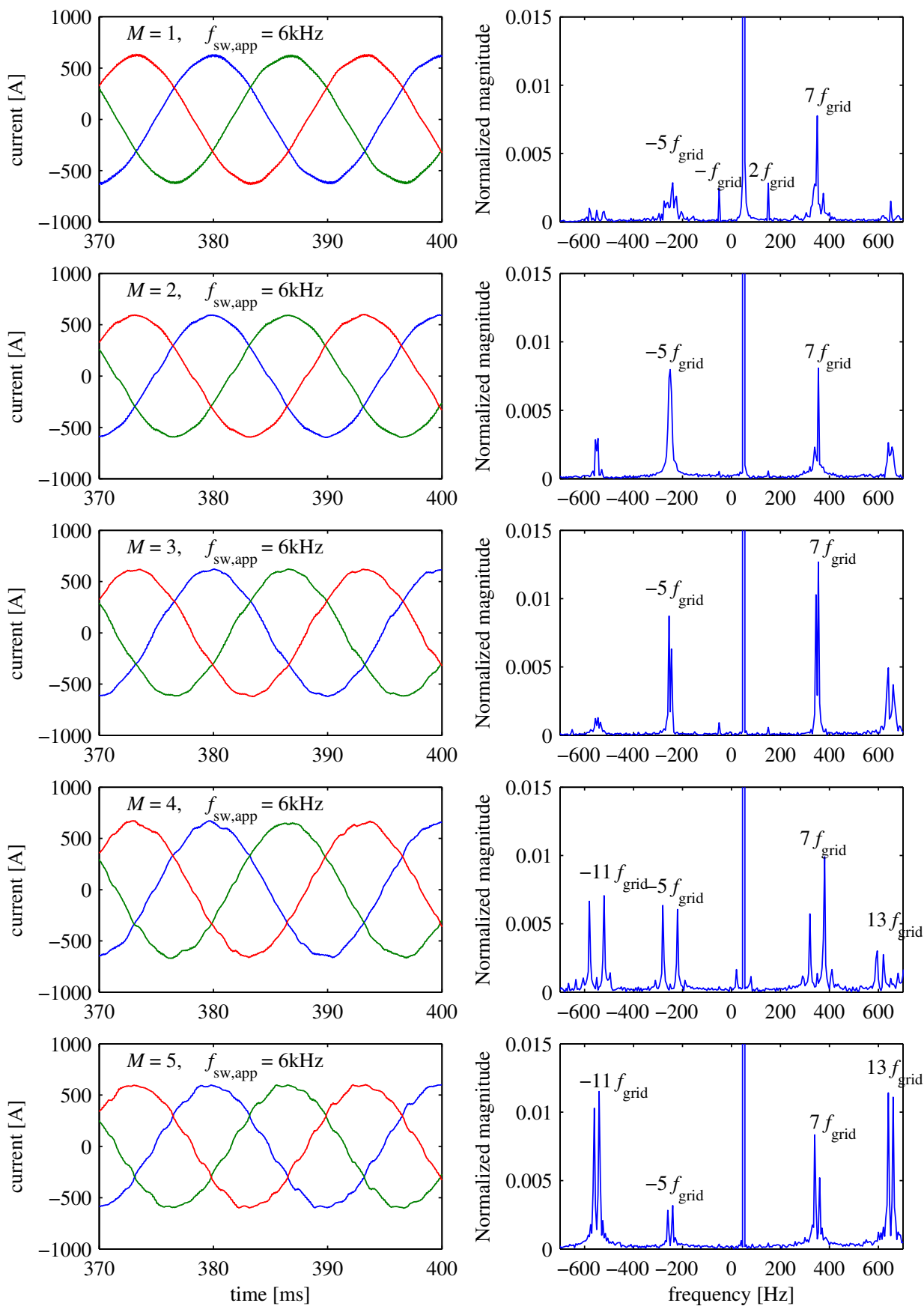

Figure 4.7. Time-domain waveforms and frequency spectra of the grid currents in the steady state at the nominal load with different numbers of cascaded belts. The apparent switching frequency of the grid inverter is $6.0 \mathrm{kHz}$ in each simulation. 

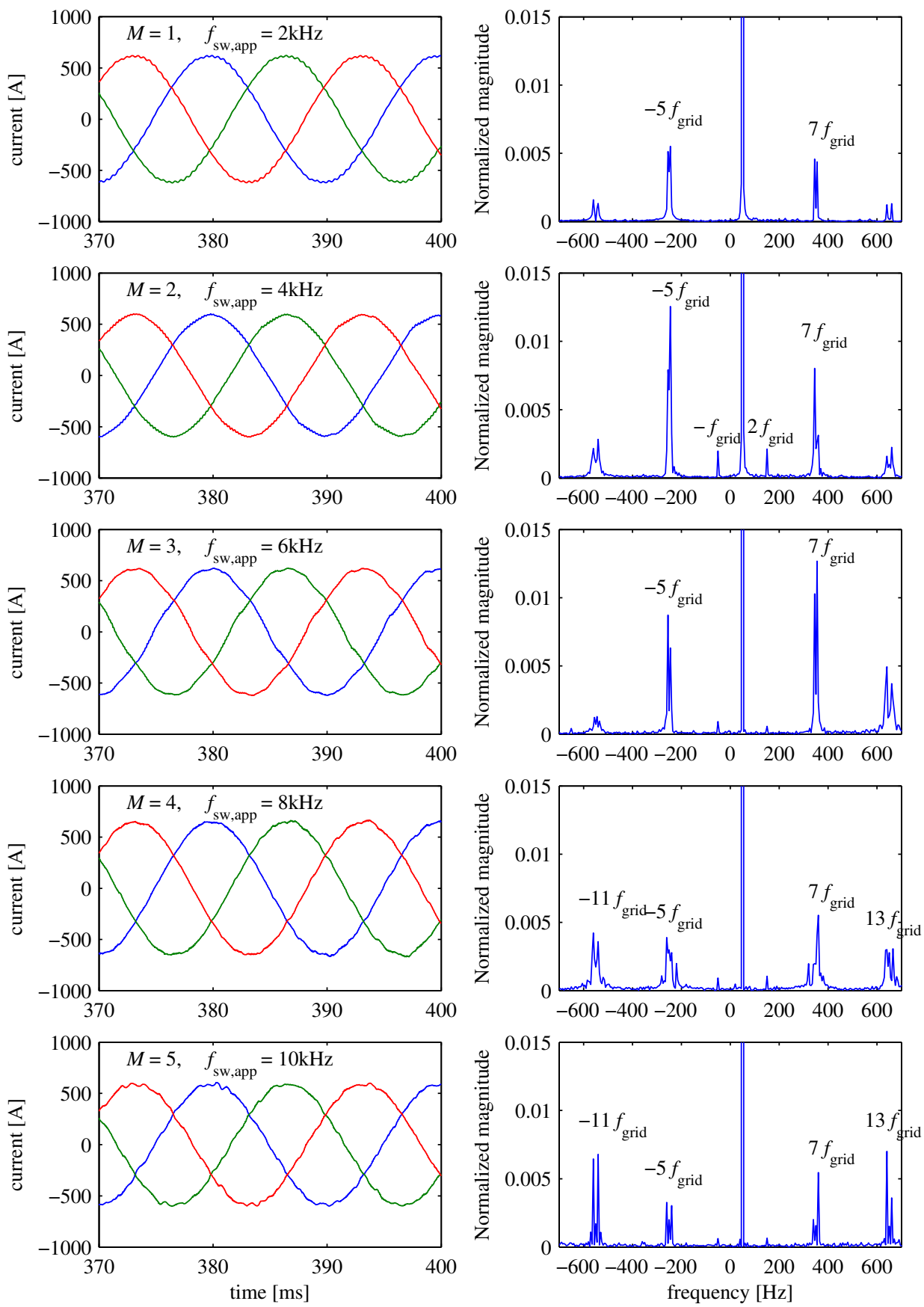

Figure 4.8. Time-domain waveforms and frequency spectra of the grid currents in the steady state at the nominal load with different numbers of cascaded belts. The switching frequency of a single IGBT of the grid inverter is $1.0 \mathrm{kHz}$ in each simulation. 
The divided spikes seen in the frequency spectra are consequences of the uncontrolled nature of the transformer energy link: Voltage fluctuation at a certain frequency on one side of isolation causes oscillations on the other side at a frequency that is a mixed result of the fluctuations on the two sides. As was discussed in Section 3.6.2, twice the fundamental frequency fluctuation is attenuated (i.e., it does not transfer from one side of the isolation to the other) because of the $120^{\circ}$ phase shift. The sixth harmonic of the fundamental, however, penetrates the transformer link.

The second simulation set corresponds better to a potential actual application of the MDC: the apparent switching frequency of the grid inverter is higher with a higher number of belts. The switching frequency of a single IGBT is fixed at $1.0 \mathrm{kHz}$, and thus, the apparent switching frequencies vary from $2.0 \mathrm{kHz}$ to $10.0 \mathrm{kHz}$ as the number of belts goes from one to five. When the results of this second simulation set are compared with the first, the following remarks are made:

1. Time-domain waveforms in the case of four and five belts have improved;

2. The locations of the dominating peaks in the frequency spectra are unchanged;

3. The magnitudes of the dominating peaks have changed:

- $M=1:$ the $-5^{\text {th }}$ has increased, the $7^{\text {th }}$ has decreased;

- $M=2:$ the $-5^{\text {th }}$ has increased, the $7^{\text {th }}$ is unchanged;

- $M=3$ : No changes because the switching frequencies match;

- $M=4$ and $M=5$ : All harmonic component peaks have lower magnitudes.

The previous comparison can be summarized as follows: the waveforms are better with $M>3$ when the apparent switching frequency is higher. However, the time-domain waveforms still include a seemingly higher distortion compared with the cases when $M<4$. If the motor supply frequency differs from the grid frequency, oscillations are generated in the voltages of the grid-side DC links. Backed with the analysis of Section 3.6.2, this behavior is a characteristic of the MDC.

The fact that the harmonic content is amplified with a higher number of belts may result from the total amount of capacitance. One belt has six DC links that are interconnected by the transformer. If the impedance of the transformer link is considered negligibly small, the DC links can be modeled as parallel-connected capacitors. Thus, the total capacitance of one belt is

$$
C_{\text {belt }}=6 C_{\mathrm{dc}}
$$

With $M$ series-connected belts, the total capacitance of the system becomes

$$
C_{\mathrm{tot}}=\frac{6 C_{\mathrm{dc}}}{M}
$$

This value can be verified by calculating the energy stored in the capacitors of the MDC. The energy stored in one capacitor is equal to

$$
E_{\mathrm{dc}}=\frac{1}{2} C_{\mathrm{dc}} u_{\mathrm{dc}}^{2}
$$



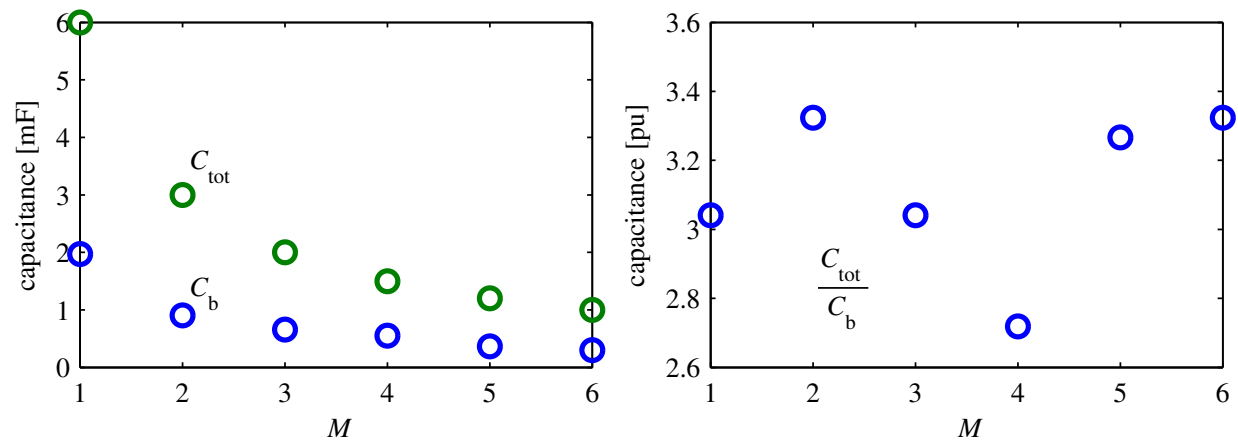

Figure 4.9. Total capacitance, base capacitance, and per-unit total capacitance of the MDC with $M$ belts.

The energy stored in all capacitors of the system is therefore

$$
E_{\mathrm{tot}}=6 M \frac{1}{2} C_{\mathrm{dc}} u_{\mathrm{dc}}^{2}
$$

Subsituting the total DC link voltage $M u_{\mathrm{dc}}$ and the total capacitance $C_{\mathrm{tot}}$ into (4.6) yields

$$
E_{\mathrm{tot}}^{\prime}=\frac{1}{2} C_{\mathrm{tot}}\left(M u_{\mathrm{dc}}\right)^{2} \text {. }
$$

Substituting (4.5) and rearranging the terms yields

$$
E_{\mathrm{tot}}^{\prime}=6 M \frac{1}{2} C_{\mathrm{dc}} u_{\mathrm{dc}}^{2}=E_{\mathrm{tot}}
$$

It is evident from (4.5) that the total amount of capacitance is inversely proportional to the number of series-connected belts. The total capacitance and base capacitance of the system are shown in Figure 4.9 as a function of $M$. As the amount of capacitance is inversely proportional to the number of belts, the voltage fluctuations are bound to increase as the power goes up. This may be the reason for the increasing magnitudes of the harmonic components when belts are added.

Finally, the study is concluded by two suggestions, which may improve the performance in practice, but will not remove the root cause of the harmonic content in the grid currents:

1. Synchronous sampling of current: the sampling instant of the currents should be concurrent with the peaks of modulator triangle waves to mitigate ripple at the switching frequency. The antialias filter is not effective enough to remove aliasing problems in the sampling.

2. An antialias filter is employed for the DC link voltage measurements to provide the feedback for the DC link voltage compensator. The cut-off frequency of this filter is $500 \mathrm{~Hz}$ regardless of the switching frequency and the number of belts. The bandwidth of this filter should be refined. 


\subsubsection{Asymmetric transformer}

Most of the simulations have been performed with a symmetric transformer, the stray impedances of which are equal among the six windings. The design and construction of this type of a transformer may prove very difficult in practice. Additionally, the manufacturing tolerances are never zero, which leads to differences between individual transformers.

The effect of an asymmetric transformer on the system performance is assessed in this section. A simulation model comprising two cascaded belts is used. The transformer of the first belt is symmetric with $L_{\sigma}=1.32 \mu \mathrm{H}$. The transformer in the second belt is asymmetric, and its stray inductance is determined by (3.49). The average values of the stray inductances in the two transformers are equal. The simulated DC link voltages are shown in Figure 4.10.

The system is in the steady state in nominal operating conditions. The average DC link voltages of belt 1 (blue and red traces) are higher compared with belt 2 . The amplitude of the ripple is also different: phase $\mathrm{A}$ in belt 2 has a clearly lower ripple amplitude than phase $\mathrm{A}$ in belt 1 . The fluctuations in phases $\mathrm{A}$ and $\mathrm{U}$ are approximately $180^{\circ}$ phase shifted, which means that most of the power from phase $A$ is transferred to phase $U$. In the case of an asymmetric transformer, the stray inductance from winding 1 to winding 4 is $1.06 \mu \mathrm{H}$ while in the symmetric transformer the inductance is $1.32 \mu \mathrm{H}$. Thus, the impedance between these two windings is approximately $30 \%$ higher in belt 1 . Thus, the ripple voltage of the corresponding DC link voltages is higher.

At $t=650 \mathrm{~ms}$, the assisted DC link voltage controller is turned on. After a short transient, the average values of the DC link voltages are equal on the primary side, but on the secondary side the average value of phase $U$ is higher in belt 1 . The numeric values of the average and ripple voltages in every DC link before and after turning on the assisted DC link voltage controller are shown in Table 4.6.

It is seen in Table 4.6 that the DC links around the symmetric transformer are always higher

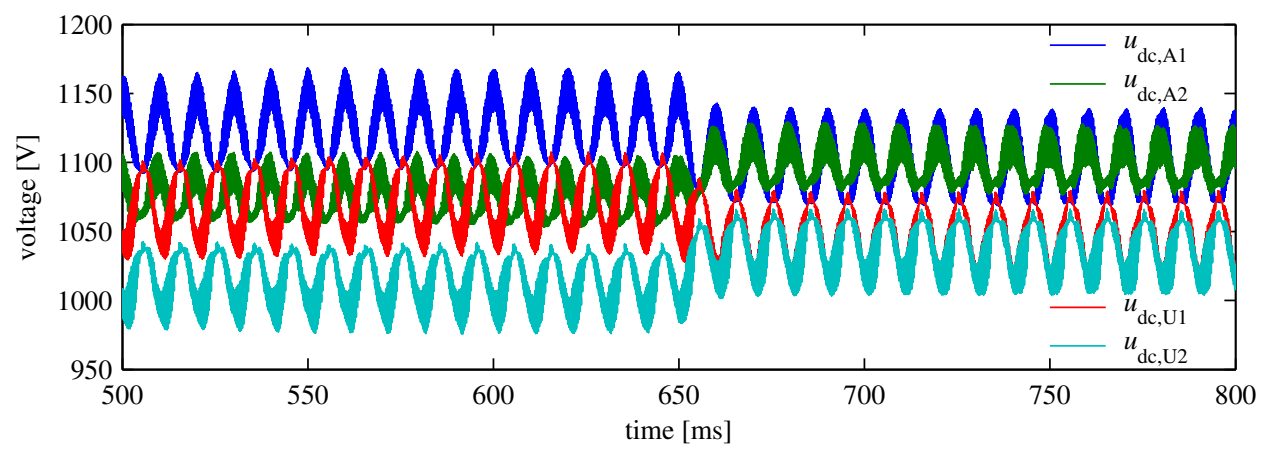

Figure 4.10. DC link voltages in two belt systems where the transformer stray impedances are different between the belts. The assisted DC link voltage control is turned on at $t=650 \mathrm{~ms}$. 
Table 4.6. DC link voltage average and ripple values in two belts in the nominal operating point when the transformer of the first belt is symmetric and the second belt is asymmetric.

\begin{tabular}{cccccc}
\hline & & $t<600 \mathrm{~ms}$ & $t<600 \mathrm{~ms}$ & $t>700 \mathrm{~ms}$ & $t>700 \mathrm{~ms}$ \\
Belt & Phase & Average [V] & Ripple [V] & Average [V] & Ripple [V] \\
\hline 1 & A & 1124 & 76 & 1098 & 71 \\
2 & A & 1076 & 53 & 1100 & 51 \\
1 & B & 1124 & 74 & 1098 & 71 \\
2 & B & 1078 & 57 & 1101 & 53 \\
1 & C & 1124 & 73 & 1098 & 68 \\
2 & C & 1077 & 64 & 1100 & 60 \\
1 & U & 1071 & 77 & 1044 & 73 \\
2 & U & 1014 & 67 & 1037 & 63 \\
1 & V & 1071 & 78 & 1044 & 75 \\
2 & V & 1014 & 55 & 1037 & 54 \\
1 & W & 1071 & 82 & 1044 & 78 \\
2 & W & 1014 & 58 & 1037 & 55 \\
\hline
\end{tabular}

on average. The assisted DC link controller adjusts the primary-side DC links very close to the reference value, but the asymmetric transformer has a higher voltage drop. The voltage drops are $54 \mathrm{~V}$ and $63 \mathrm{~V}$ for the symmetric and asymmetric transformers, respectively.

This study is concluded by the following remarks:

1. The average values of the DC link voltages on one side of the isolation in one belt are equal even with an asymmetric transformer;

2. All DC link voltages of the belt with a higher voltage drop are lower on average compared with the other belt;

3. The imbalance of the primary-side average DC link voltages between the belts can be corrected with the assisted DC link voltage controller;

4. A higher impedance of the transformer link results in a higher ripple voltage, which is not balanced by the assisted DC link voltage controller.

\subsubsection{Seventh winding in the transformer}

The MDC as a whole could benefit from the addition of a seventh winding to the mediumfrequency transformer. This hardware modification could serve multiple purposes, some of which are listed below.

1. Voltage balancing/energy exchange between the belts;

- The $7^{\text {th }}$ windings of each belt transformer are connected in parallel.

- Provides an isolated route for energy exchange between belts.

2. Location of a brake chopper;

- Brake choppers are compulsory in applications that require continuous braking torque 
even in the case of a network blackout.

3. Sense winding for the magnetic state of the transformer;

- The $7^{\text {th }}$ winding is equipped with voltage measurement instrumentation, which, together with a processor, provides the feedback signal required by the antisaturation control of the transformer; see (Sankala et al., 2014a).

- This functionality cannot be operational concurrently with voltage balancing or brake chopper application.

These advantages motivate the research on the applicability of the seventh winding. The third item, namely the feedback signal for the antisaturation control, is omitted here, because the control algorithm is not studied further in the scope of this work. The first two items, that is, the voltage balancing between the belts and the brake chopper, are analyzed in the following by simulations.

The six main windings have to be as symmetric as possible to obtain a balanced voltage of each submodule inside a belt. The starting point for the design of the seventh winding is an assumption that this winding does not need to be a symmetric part of the transformer. When it comes to voltage balancing between the belts, current will flow from one transformer to another if the induced voltages are different. The actual value of the stray impedance sets the magnitude of the current and the time constant for the voltage balancing. The stray impedance of the seventh winding has practically no effect on the operation of the brake chopper if the chopper is implemented as shown in Figure 4.11.

If the transformer is constructed with an E-core with the main windings wrapped around the central leg, the seventh winding can be distributed evenly on the outer legs of the core. By applying this method, the addition of the seventh winding will not compromise the design of the symmetric main windings. The number of turns in the seventh winding is chosen equal to the main windings. Thus, low-voltage IGBTs can be used for the brake chopper.

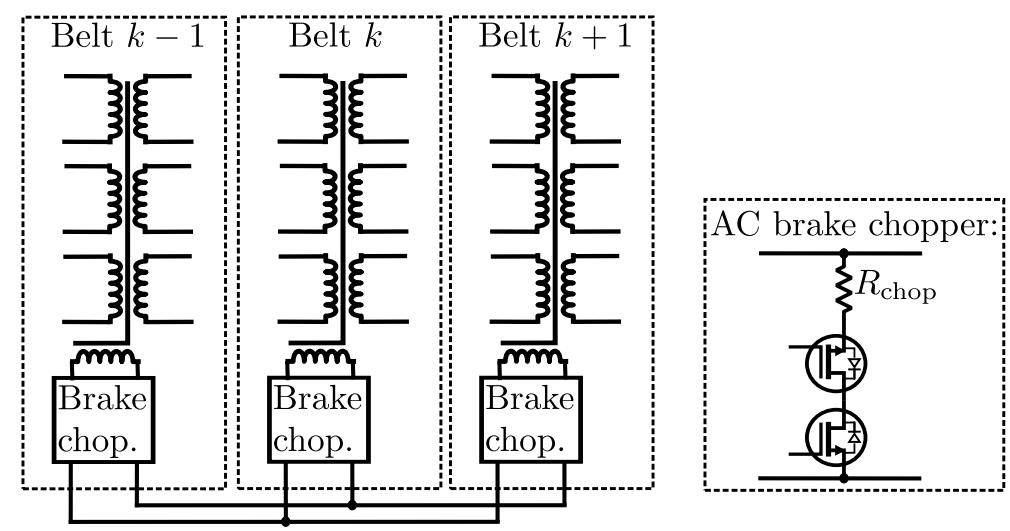

Figure 4.11. Circuit diagram description of the seventh winding with a brake chopper and the interconnection of belts. 
The performance of the system with the seventh winding is assessed by simulations of a two-belt system. Two simulations are performed:

1. Voltage balance during a load power sign change and brake chopper operation with a negative load power in the case of a network blackout, and

2. Voltage balance of the belts is observed in a multiport application and compared with the results shown in Section 3.5.4.

Addition of the seventh winding modifies the stray impedance matrix; see (3.49). The size of the new matrix is $7 \times 7$. The simulations are performed with the following matrix, where the main windings are symmetric, and the inductance and resistance of the seventh winding are double compared with the main windings.

$$
\mathbf{L}_{\sigma}=\left[\begin{array}{ccccccc}
0 & 1.32 & 1.32 & 1.32 & 1.32 & 1.32 & 2.64 \\
1.32 & 0 & 1.32 & 1.32 & 1.32 & 1.32 & 2.64 \\
1.32 & 1.32 & 0 & 1.32 & 1.32 & 1.32 & 2.64 \\
1.32 & 1.32 & 1.32 & 0 & 1.32 & 1.32 & 2.64 \\
1.32 & 1.32 & 1.32 & 1.32 & 0 & 1.32 & 2.64 \\
1.32 & 1.32 & 1.32 & 1.32 & 1.32 & 0 & 2.64 \\
2.64 & 2.64 & 2.64 & 2.64 & 2.64 & 2.64 & 0
\end{array}\right] \times 10^{-6}[\mathrm{H}]
$$

Gate pulses for the two IGBTs of the brake chopper are generated by a simple hysteresis block. If the average DC link voltage on the grid side exceeds $1300 \mathrm{~V}$, both IGBTs are turned on. Once the voltage level goes below $1200 \mathrm{~V}$, the switches are turned off. The simple logic was selected to obtain a proof-of-concept simulation. The resistance of the chopper is selected as $R_{\text {chop }}=1.0 \Omega$.

The first simulation starts from the nominal operating point, $P_{\mathrm{mot}}=1.5 \mathrm{MW}$. At $t=150 \mathrm{~ms}$, the external torque of the load is reversed with a limited ramp while the motor controller acts to maintain a constant speed. A negative nominal torque is reached at $t=350 \mathrm{~ms}$, and a steady state is attained after a short transient produced by the motor controller. At $t=600 \mathrm{~ms}$, a grid blackout occurs: all phase voltages go to $0 \mathrm{~V}$. At the same time, the grid-side modulator is bypassed, and each IGBT of the grid inverter is turned off. The operating point is not changed during the last $400 \mathrm{~ms}$ of the simulation.

The motor torque and speed are shown in Figure 4.12. The initialization transient of the simulation is not over before the external torque starts descending towards a negative value. During the torque reversal, the speed of the motor increases to approximately $1530 \mathrm{rpm}$, but shortly after the torque has assumed a constant negative value, the speed settles to the reference value, $1486 \mathrm{rpm}$. The motor changes from the motoring mode to the generator mode at $t \approx 250 \mathrm{~ms}$. The grid blackout, which occurs at $t=600 \mathrm{~ms}$, has no effect on the mechanical operation of the motor.

The voltage balance and current needed to balance the two belts are depicted in Figure 4.13. The DC link voltages on one side of the isolation are exactly on top of each other, which means that balancing is very effective. The current needed for the balancing to take place is 
only $35 \mathrm{~A}_{\text {peak }}$ and $17 \mathrm{~A}_{\mathrm{RMS}}$. The RMS value is calculated from a time span of $400 \mathrm{~ms} \leq t \leq$ $500 \mathrm{~ms}$.

The operation of the brake choppers is shown in Figure 4.14, where the time span of $550 \mathrm{~ms}$
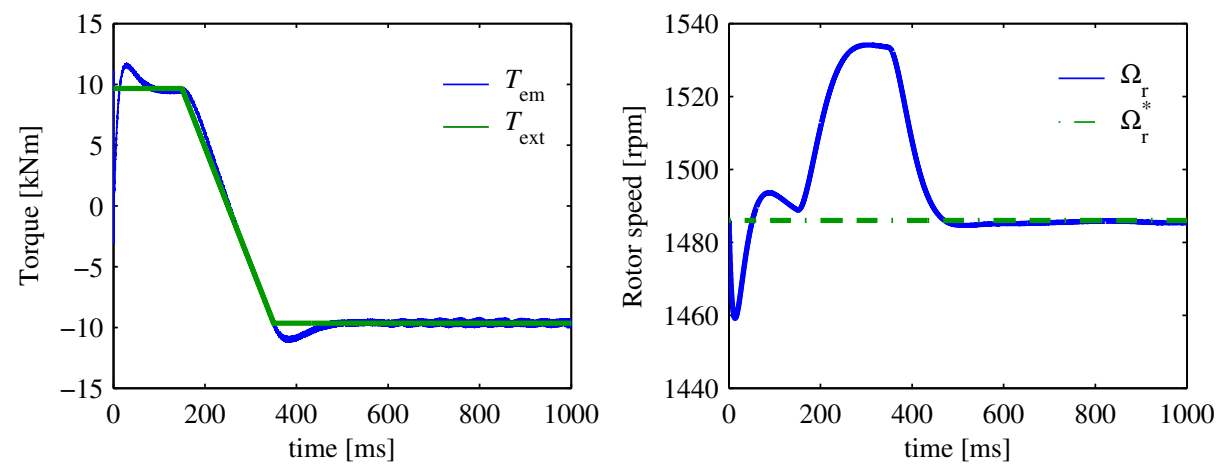

Figure 4.12. Motor torque (external and electromagnetic) and rotor speed in the simulation with the seventh winding and the brake chopper.
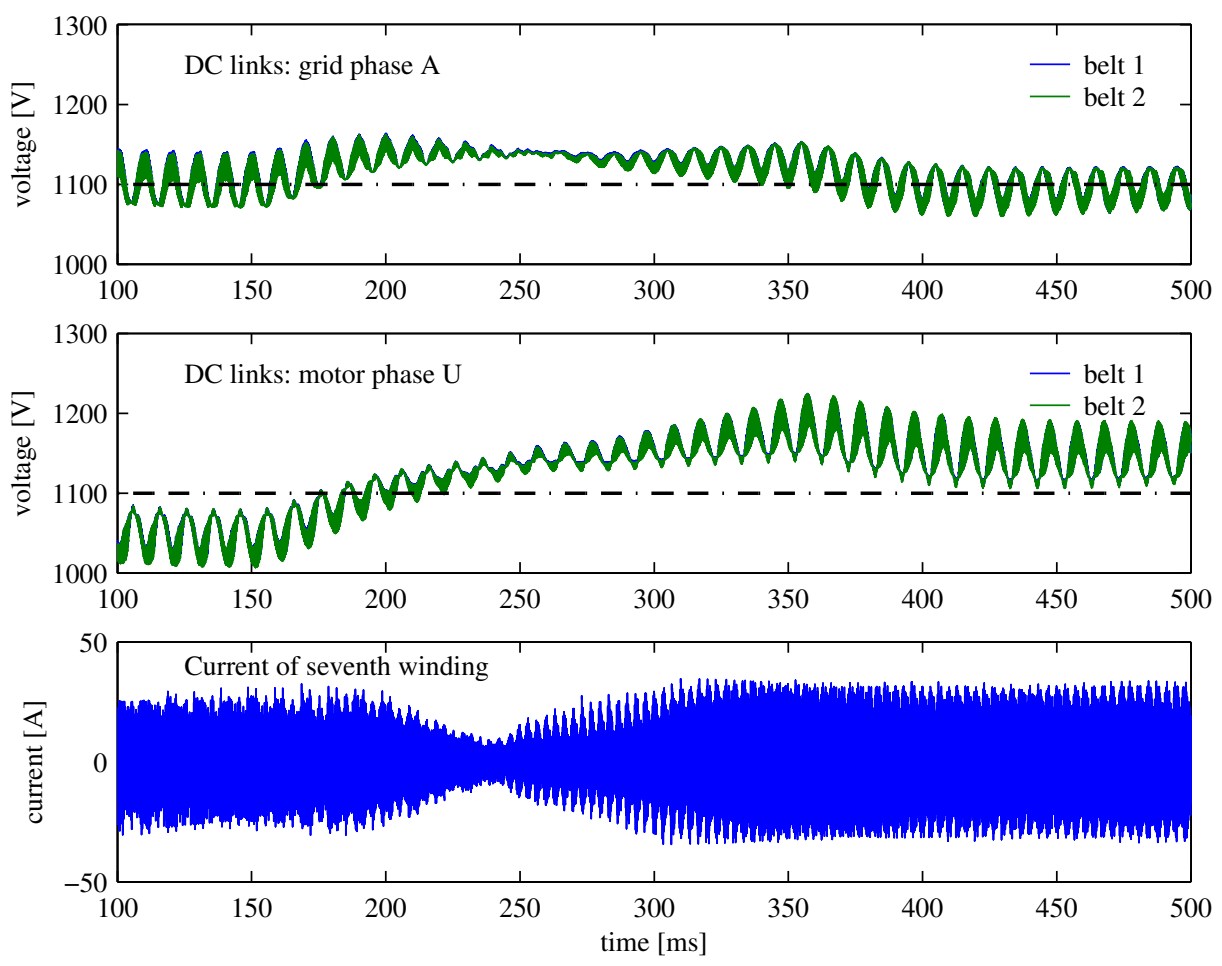

Figure 4.13. DC link voltages and current of the seventh winding during the power sign reversal. 

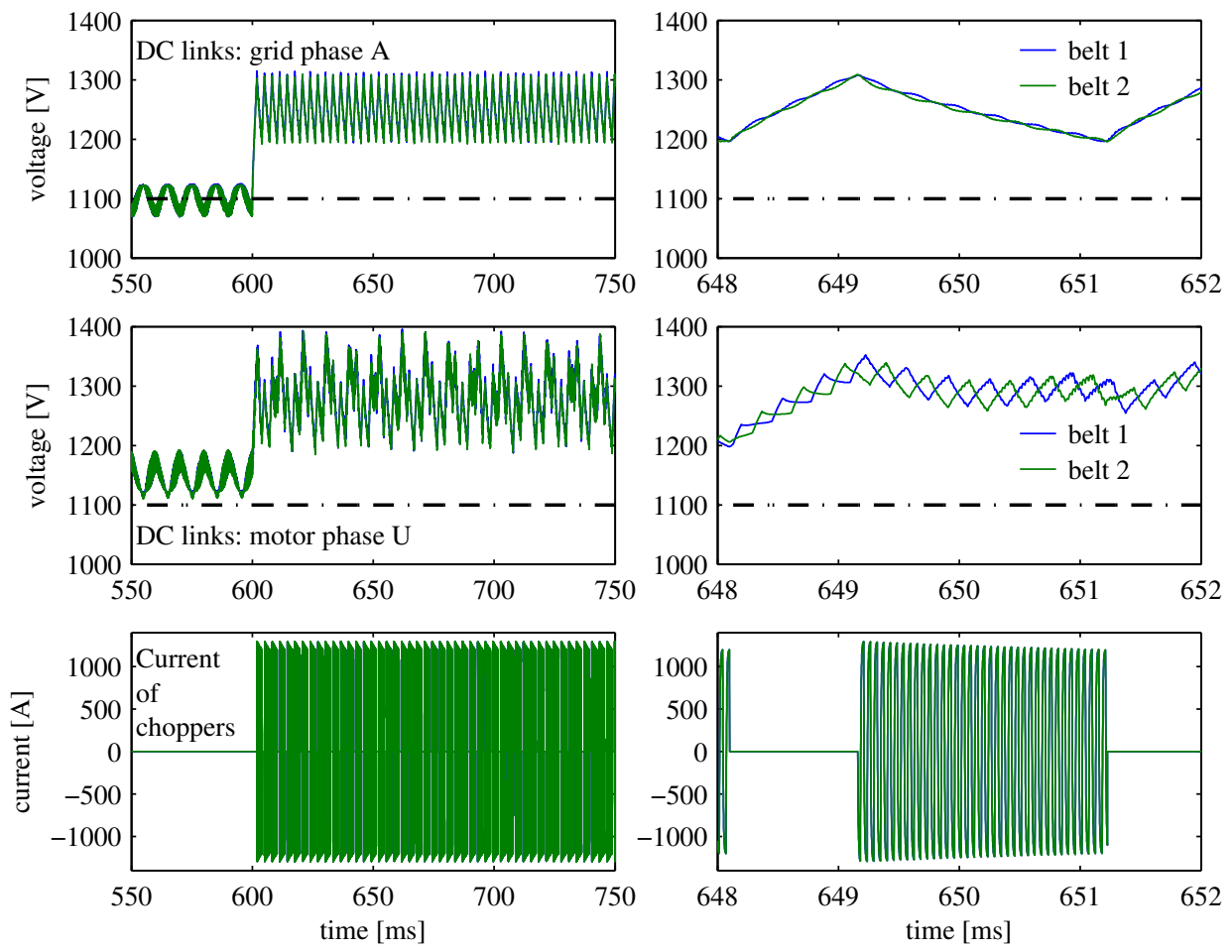

Figure 4.14. Operation of the brake choppers during a network blackout. A zoom-in figure on the right.

$\leq t \leq 750 \mathrm{~ms}$ is shown on the left and a focused time span $648 \mathrm{~ms} \leq t \leq 652 \mathrm{~ms}$ on the right. The DC link voltages jump from $1100 \mathrm{~V}$ to $1200 \mathrm{~V} \ldots 1300 \mathrm{~V}$ when the network blackout starts. The current starts to flow through the brake chopper resistors: the peak value of current is $1300 \mathrm{~A}$ and the RMS value is $830 \mathrm{~A}$. The two brake resistors dissipate the load power, which means that the two resistors are quite large objects in practice and have to be cooled effectively. It is clear that these resistors and their cooling system cannot be included in the mechanical construction of the belt. Instead, they are separate objects located outside the main converter structure, which is a common practice in the industry.

The DC link voltages are kept within the hysteresis band on the primary side, while on the secondary side they occasionally reach $1400 \mathrm{~V}$. A better design of the hysteresis bands is required. The feedback signal should also be formed from the average of the secondary-side DC link voltages, because the power is negative and the voltages on the secondary side are higher.

The second simulation shows the advantage of having a seventh winding in a multiport application. The simulation of Section 3.5.4, Figure 3.32, is repeated with the additional winding. Figure 4.15 shows the electromagnetic torques and speeds of both motors. Smooth operation is obtained during and after the braking of motor 2 . The torque current reference was lim- 

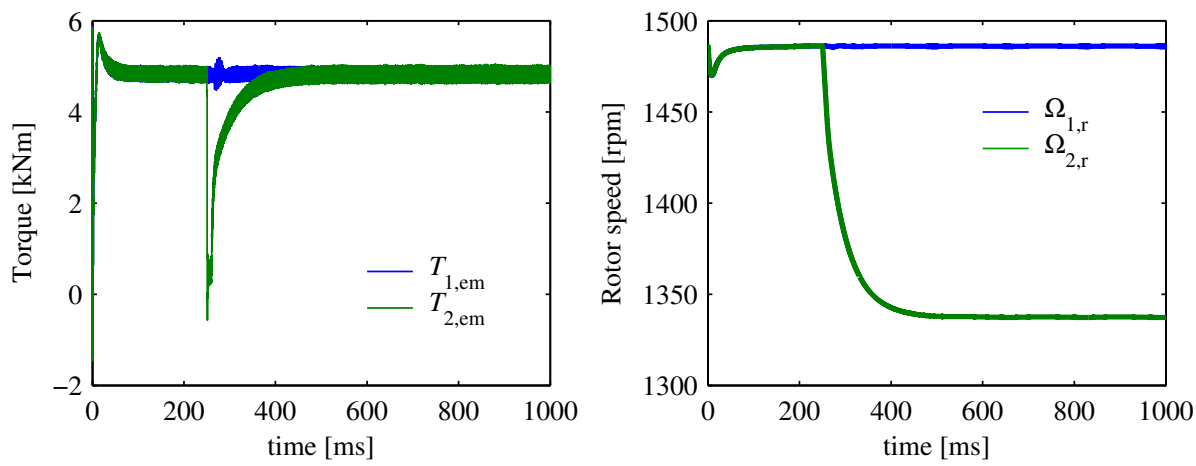

Figure 4.15. Electromagnetic torque and rotation speeds of the two motors in a multiport application when the belts are coupled through the seventh winding.

ited to $0.05 \mathrm{pu}$ so that the sign of the power does not change during the transient. This was done because the operation of the assisted DC link voltage controller is not satisfactory with negative power; see Section 3.4.4.

The DC link voltages in the multiport application are shown in Figure 4.16. Voltage ripple occurs during the transient caused by braking of the second motor, but the performance is superior compared with the case where the assisted DC link voltage controller was the only element providing regulation of the voltages; see Figure 3.32. The current of the seventh winding is observed to reach quite a high peak value during the transient: $760 \mathrm{~A}$. However, the RMS value is low because the transient of the DC link voltages is over in less than $100 \mathrm{~ms}$. Note that the brake choppers were disabled in this simulation.

The presented simulation results showed that the seventh winding is an effective balancing method for the DC link voltages between belts. The added winding can also be employed in an application that requires a brake chopper. However, these two applications present considerably different specifications to the design of the seventh winding. This is evident for the current rating of the seventh winding. In normal operation, the current required to balance the belts is in the range of tens of amperes. The RMS value of the current required for the balancing in the multiport application is also low, but the peak value is relatively high. In the case of a brake chopper, the RMS value of the current is nearly $1000 \mathrm{~A}$ because the brake resistor has to dissipate the nominal power of the belt. The design of the seventh winding for the brake chopper application allows it to be used in all applications presented here. The seventh winding was implemented with $100 \%$ higher values for stray inductance and resistance compared with the main windings, and the desired functionality was obtained. This verifies the initial assumption that the seventh winding does not necessarily have to be a symmetrical part of the transformer. Even though not mentioned before in this section, the balancing provided by the seventh winding is predicted to help mitigate circulating currents when the belts are connected in parallel, because the voltage difference between the belts will be smaller. 

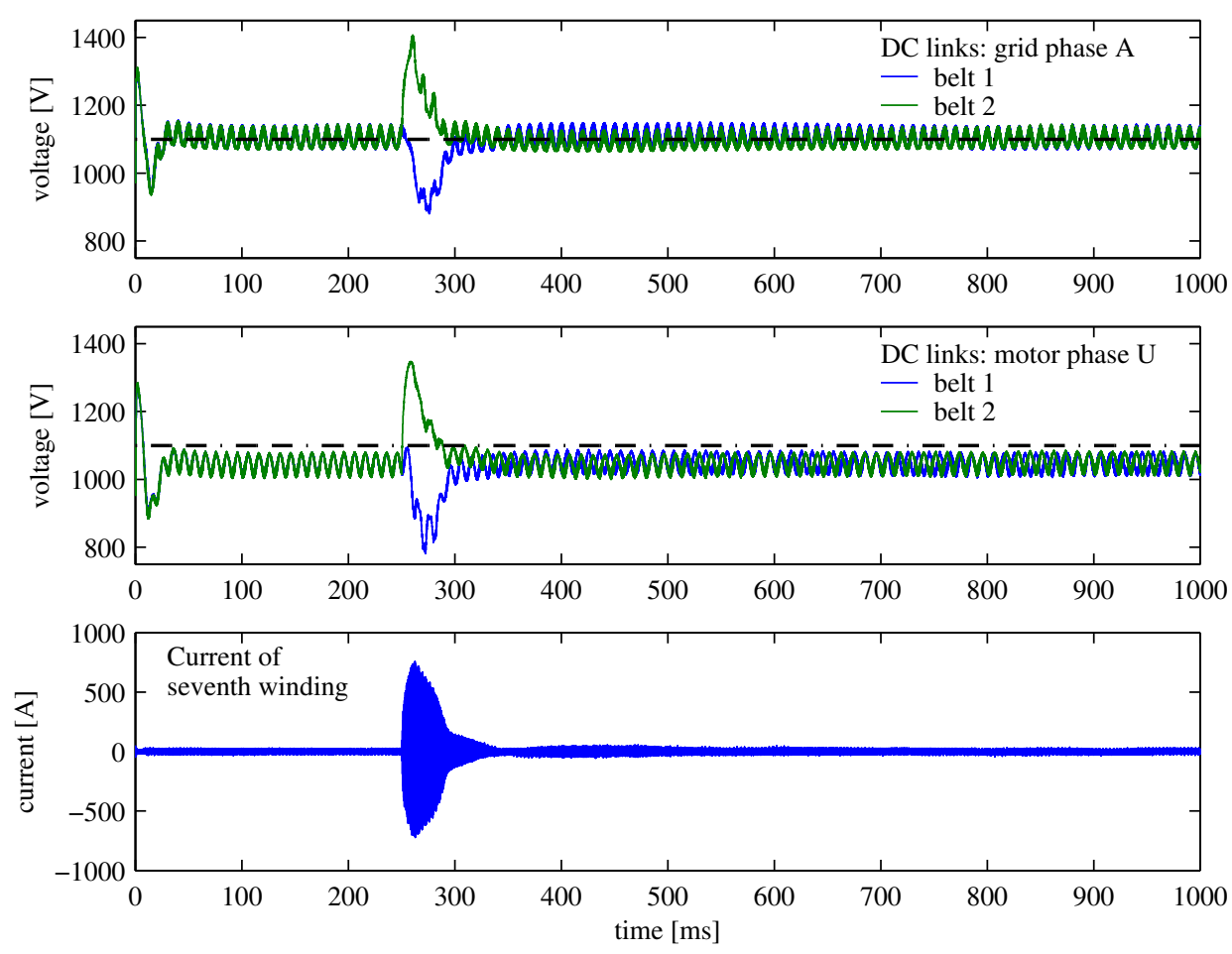

Figure 4.16. DC link voltages and current of the seventh winding in a multiport application with two motors.

\subsubsection{Common-mode voltages and currents}

Inverters are known to produce common-mode $(\mathrm{CM})$ voltages that cause $\mathrm{CM}$ currents. These currents are AC by nature and cause wear-out for example in motor bearings (Chen et al., 1996). Therefore, minimization of the CM voltage is of utmost importance. Many contributions have studied the relation of the modulation method and the common-mode voltage. For example, Naumanen (2010) compared three modulation methods in a CHB application.

The CM voltage is the average of the three phase voltages produced by the inverter. Therefore, the instantaneous value of CM voltage can be calculated by

$$
u_{\mathrm{CM}}(t)=\frac{1}{3}\left(u_{\mathrm{U}}(t)+u_{\mathrm{V}}(t)+u_{\mathrm{W}}(t)\right)
$$

The amount of CM voltage in the MDC is not expected to differ from a normal CHB inverter when similar modulation algorithms are used. The production of common-mode voltage was studied with different numbers of belts and in two operating conditions. In the first operating condition, the motor is operated at $50 \mathrm{~Hz}$ (nominal speed), and in the second condition, the 

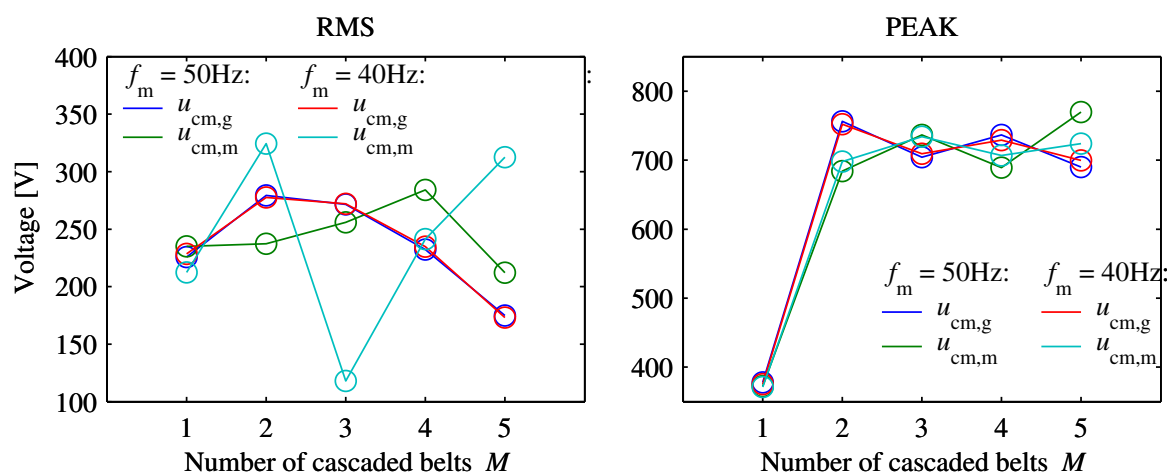

Figure 4.17. Common-mode voltages produced by the grid and the motor inverters under two different loading conditions as a function of number of belts.

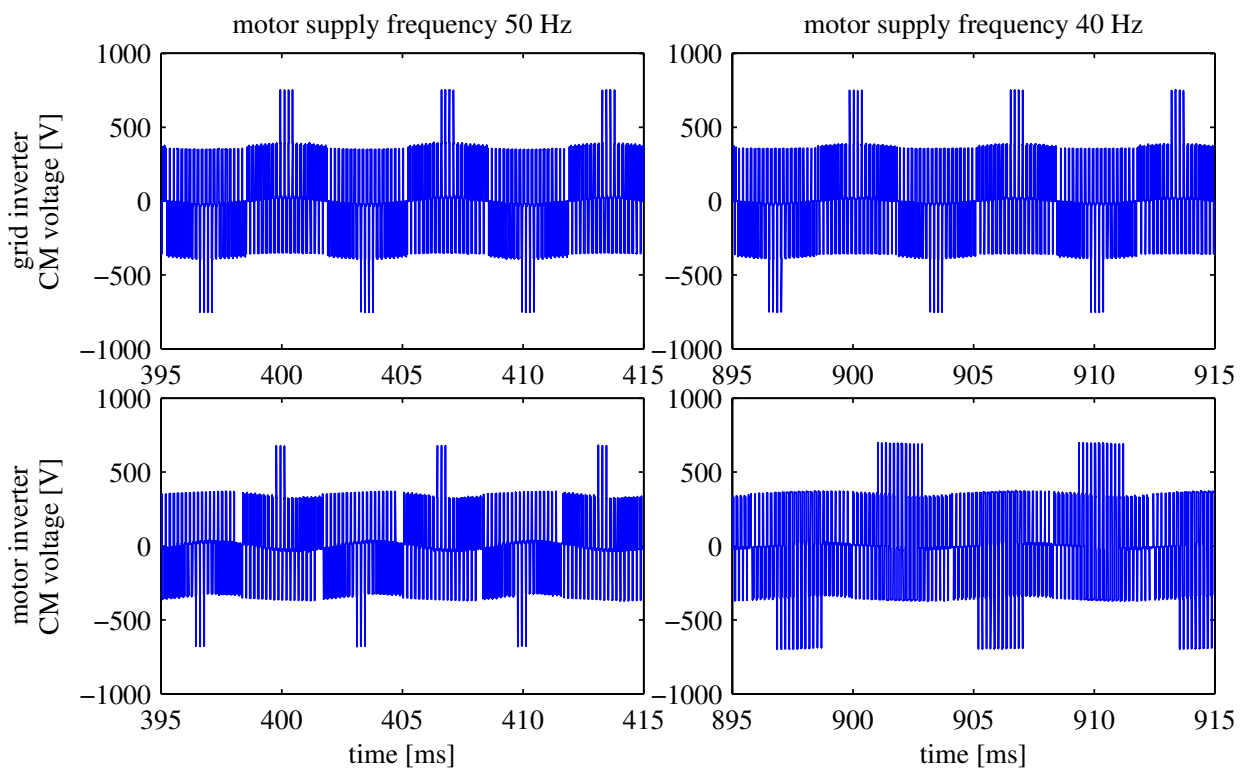

Figure 4.18. From top: Common-mode voltage of the grid inverter and the motor inverter. $50 \mathrm{~Hz}$ and $40 \mathrm{~Hz}$ motor supply frequencies are applied in the left and right subplots, respectively.

motor is operated at $40 \mathrm{~Hz}$. The resulting CM voltage RMS and peak values are presented in Figure 4.17. The CM voltage time-domain waveforms in both operating points with two belts are shown in Figure 4.18. It is seen that the CM voltage does not increase with a higher application voltage rating, where more belts are needed. Two exceptions are present: With one belt, the peak value of the CM voltage is half of the peak value when $M \geq 2$. With three belts and a $40 \mathrm{~Hz}$ motor supply frequency, the RMS value of the CM voltage is significantly lower compared with the other measured points. 


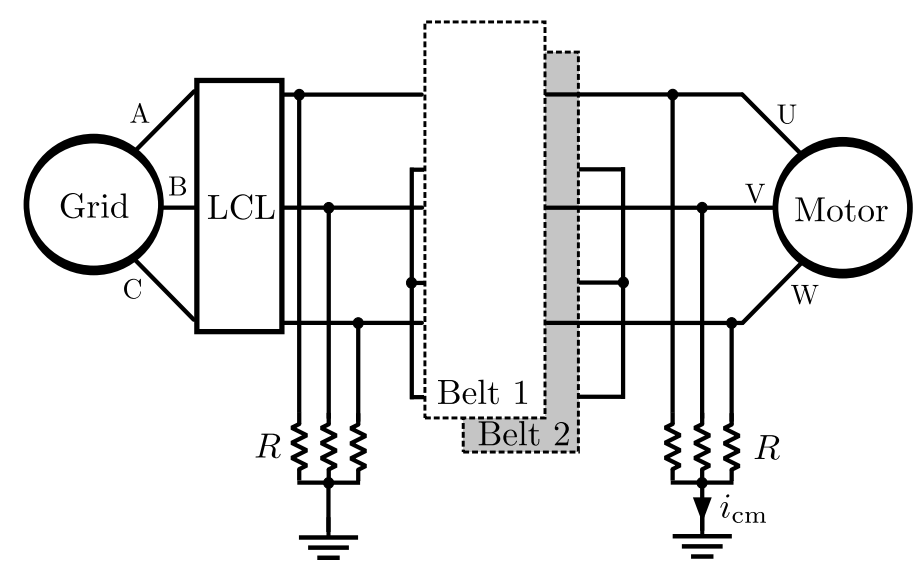

Figure 4.19. Artificial star points are created on both sides of the MDC by connecting a resistor from each line to the ground.

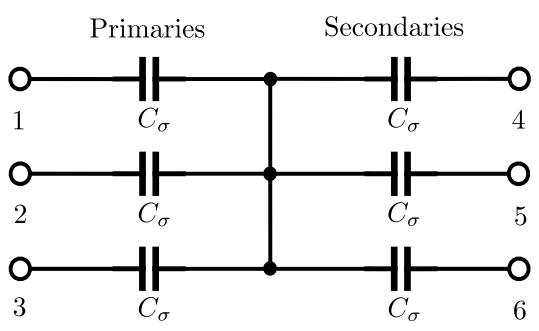

Figure 4.20. Capacitive couplings of the six transformer windings are modeled with six capacitors. The capacitive transformer model is connected in parallel with the inductive transformer model by connecting the positive winding end of each winding to one of the capacitors.

Common-mode current is studied with simulations of a two-belt MDC operating in the nominal loading condition in the steady state. In order to measure the CM current, two modifications to the simulation model were made. First, artificial star points were added on both sides of the converter as shown in Figure 4.19. Second, a model of stray capacitances in transformer windings was applied, see Figure 4.20. The star points are provided by resistors with $500 \Omega$ resistances. Normally the coupling from line to ground is capacitive, but here resistors are used for simplicity. The stray capacitance between two windings is selected as $10 \mathrm{nF}$, and thus, the value of a single capacitor of the circuit model is selected as $C_{\sigma}=20 \mathrm{nF}$ (two capacitors in series provide the path between two windings).

The simulated common-mode waveforms are shown in Figures 4.21 and 4.22. The first figure shows a practically zero CM current, because the stray capacitance model was not applied. The transformers in the MDC provide effective CM current filtering by cutting the return path of the current. However, when the stray capacitances of the windings are included in the simulation model, the CM current is nonzero. The CM current shown in the second figure has a peak value of $4.3 \mathrm{~A}$ and an RMS value of $0.65 \mathrm{~A}$. The repetitive short spikes seen in the CM current waveform recur at the transformer operating frequency, $18 \mathrm{kHz}$. The selected stray 


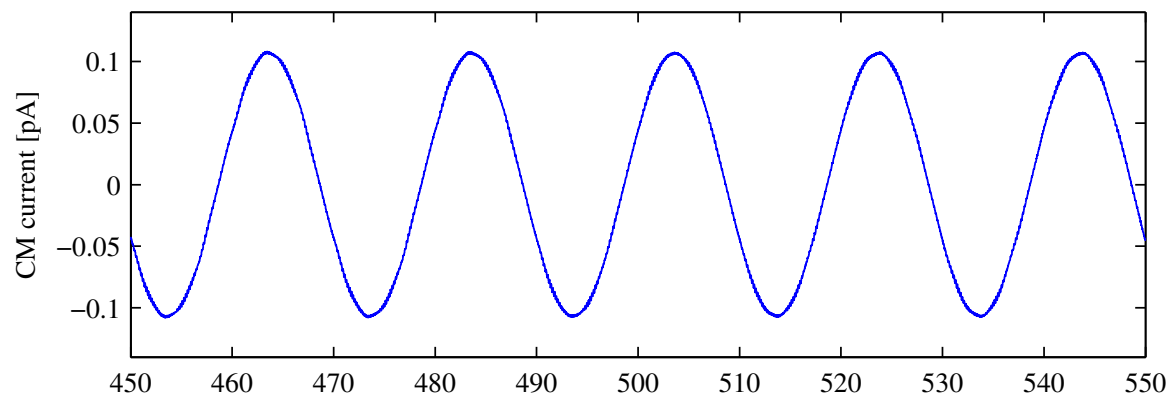

Figure 4.21. Common-mode current $i_{\mathrm{CM}}$ when the transformer stray capacitances are not included in the simulation model.

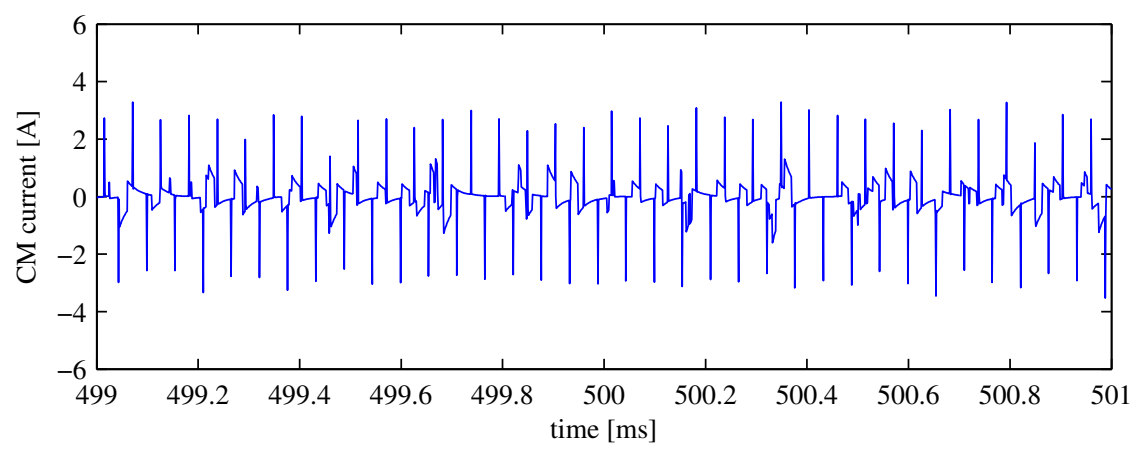

Figure 4.22. Common-mode current $i_{\mathrm{CM}}$ when the transformer stray capacitances are included in the simulation model.

capacitances and line-to-ground resistances only provide means to assess the transformer's capability to filter CM current. The actual values of the current are not relevant here.

The simulation results show that the stray capacitance of the transformer provides a path for the common-mode current. The transformer switching operation is clearly visible in the simulated common-mode current, which is an unexpected result. Future research should focus on validating the simulation result by experiments.

\subsection{Summary of the feasibility study}

This chapter explored the technical feasibility of the MDC. The research was divided into two separate parts: In the first part, the hardware requirements of the MDC were compared with 
other topologies. The topologies were selected among the ones that were analyzed in Chapter 2. The comparison showed that the topologies have significantly different hardware configurations in terms of the number of passive and active components. As expected, the MDC and the UNIFLEX-PM were quite similar, with the major difference being in the number of brake choppers needed. Whereas the UNIFLEX and the CHB need nine, the MDC copes with three brake choppers. The footprints of the medium-frequency transformers of the MDC and the UNIFLEX-PM were also compared. The 5LANPC provided a good compromise between the number of voltage levels and the component count. However, the topology requires IGBTs with $2 \mathrm{kV}$ and $4 \mathrm{kV}$ voltage ratings, whereas the other VSI topologies need $1700 \mathrm{~V}$ components.

The second part of the section addressed various technical aspects of the MDC. An integral part in each studied detail was the six-winding medium-frequency transformer. The study was started by investigating the voltage stress experienced by the transformer. It was pointed out that transformers in different belts face different amounts of voltage stress. In order to obtain a better balance in the voltage stresses of different transformers, a relocation of the neutral point was suggested. Then, the RMS and peak values of the voltage stress were simulated in the cases of one to five cascaded belts. It was observed that the RMS and peak values are dictated by the grid voltage and the DC link voltage, respectively. The maximum operating voltage of the MDC is restricted below $7 \mathrm{kV}$ (line-to-line) if the voltage blocking capability of the prototype transformer is not increased in the potential final design.

Next up was the performance in overloading conditions. The stray inductance of the transformer produces a load-dependent voltage drop. It was found out that a $180 \%$ load power produces too high a voltage drop: the speed of the motor cannot be maintained without reducing the operating voltage, that is, going into flux weakening even when the speed of the motor is nominal or under the nominal value. It was shown by simulations that a lower inductance mitigates the problem. Thus, minimization of the stray inductance in the transformer design is of very high importance.

The input current quality was studied next. Even though the number of voltage levels in the produced voltage increases with the number of belts, the quality of voltage may be degraded. It was shown that a higher apparent switching frequency does not mitigate this problem. The problem is a characteristic of the MDC resulting from the uncontrolled nature of the transformer energy link. One factor that may cause the problem to amplify with a higher number of belts is the lower total effective DC link capacitance.

The fourth technical aspect studied in this section was the effect that asymmetric transformer leakage impendaces and tolerances between different transformer units have on the system performance. It was observed that DC link voltages differ by average and ripple voltage values between belts with slightly differently parametrized transformers. The difference in the average values of the grid-side DC link voltages can be compensated by using the assisted DC link voltage controller. However, this controller cannot help with different effective voltage drops of the transformer, and as a consequence, the average values on the motor side are not equal. This problem causes slight deterioration in the voltage quality on the motor side, but as the difference in the average DC link voltages is below ten volts, this is not expected to 
pose problems in a practical application. This statement applies to cascaded belts, but when belts are connected in parallel, unequal DC link voltages give rise to circulating currents.

The second to last section presented a hardware modification of the transformer. Through the addition of a seventh winding, the voltage balance problem described in the previous paragraph can be mitigated. The belts can exchange energy when the seventh windings are connected in parallel. This method also provides means to overcome the high DC link voltage ripple present in the transient state of multiport applications. Furthermore, a brake chopper can be installed on the seventh winding, which increases the modularity of the system. However, the current rating of the seventh winding must be chosen such that the nominal power of the belt can be safely driven through one winding.

The final technical aspect considered in this chapter was the common-mode voltage and current produced by the MDC. Again, the RMS and peak values of the CM voltage on the grid and motor sides were defined as a function of number of cascaded belts. It was seen that with the chosen modulation method, the peak value of the CM voltage is $\frac{2}{3} u_{\mathrm{dc}}$. The amplitude stays below $1 \mathrm{kV}$, even when the DC link voltage fluctuation is taken into account. This result was expected because similar behavior is obtained with the CHB inverter. The simulations of the common-mode current revealed that current has no return path in the ideal case, but when stray capacitance of the transformer windings are included in the model, the common-mode current gets a nonzero value. 


\section{Chapter 5}

\section{Discussion}

In the introduction of the doctoral dissertation, three questions were presented. These research questions are answered in the following paragraphs with remarks that rely on the research conducted.

The first question was answered immediately: The modular multilevel converter, which employs low-voltage power modules and "gets the green flag" in intellectual property issues, is the proposed new topology: the modular double-cascade converter.

Next, the applicability of the existing low-voltage products to construct an MDC was in question. The following assumption has been persistent throughout the research: identical power modules are used to construct each energy conversion stage. This assumption is beautiful, yet unpractical. As presented in various parts of the dissertation, the peak value of the current of the transformer-connected H-bridges is $200-300 \%$ of that in the grid or motor bridges in nominal operating conditions. Further, in Section 4.2.2, it was shown that with a $200 \%$ load, the peak values of the transformer currents are nearly $1000 \%$ of the nominal peak current of the other bridges. In practice, this means that the current rating has to be selected according to the transformer bridges, which makes the grid and motor bridges overrated if similar building blocks are used. A more practical solution is to select a higher current rating for the transformer connected bridges.

Furthermore, the construction of the prototype taught that special care has to be put on the mechanical design to minimize and balance the stray impedances of the transformer link. The effect of unbalanced impedances was further investigated in Section 4.2.4. In order to minimize the impedances and to build belts as close to identical as possible, the mechanical design should be an integrated one, where the distance of the H-bridges and the transformer is as short as possible, and the distances from all cells to the transformer are equal. This kind of a construction is very difficult to provide with off-the-shelf inverter products and the six-winding transformer. 
The third question relates to the credibility, reliability, and investment cost of the converter. There is no straight answer, but some educated speculations can be made. The MDC shows some good features for medium-voltage drives, such as high modularity, small footprint, high input and output power quality, and voltage transformation capability. However, the bulk of the actual industrial applications do not require any of these properties. Converters with 4Q capability are often referred to as low-harmonic drives or premium drives in the industry. These drives are usually more expensive than their 2Q counterparts. Many applications such as fans do not require 4Q operation. The MDC is an unattractive solution for this kind of an application because an active front end and a grid filter are required. Without an AFE, that is, when diode bridges are used, the harmonic content of grid currents is very high.

Other topologies such as the CHB and variants of the NPC and the FC apply a phase-shifting transformer and multipulse rectification to reduce the harmonic content in $2 \mathrm{Q}$ applications. This kind of a grid configuration could be used in the MDC also. In this setup, the grid frequency phase-shifting input transformer would comprise $M$ low-voltage three-phase secondaries, each connected to the three-phase input of one belt. The grid sides of the belts are not wired together, but a cascaded connection of the H-bridges would be provided on the medium-voltage motor side. This configuration of the MDC includes two separate isolation stages adding cost and losses to the system. It also removes certain advantages such as small footprint. Therefore, it is unlikely that the MDC could compete with the other topologies in $2 \mathrm{Q}$ applications. In addition, the component count is significantly higher in the MDC compared with the other topologies.

Next, some future research subjects requiring additional effort are presented. Even though Chapter 3 provided a comprehensive analysis of the grid filter and AFE controller design, future research on this topic should focus at least on the following:

1. Study on the applicability of a simple L-filter to simplify the grid interface;

2. Performance of the AFE controller and the MDC in grid fault conditions;

3. Development of the assisted DC link voltage controller to overcome the issues encountered with reversed load power.

Issues related to the motor control are not topology-specific. Therefore, motor control development does not come under the research project of the MDC. However, the load connection principles and the voltage transformation issues presented in Chapter 3 call for further study:

1. Optimization of the output inductance value in the case of parallel and delta connections;

2. Can an assisted DC link voltage controller be used on the motor side also?

- DC link unbalance on the motor side between belts is produced when the transformer stray impedances are unequal among the belts.

- Is the addition of a seventh winding a more rational solution to the voltage unbalance issue?

When talking about the advantages of the MDC, the innovative transformer link is brought 
up: it provides isolation of the modules, common-mode filtering, and modularity, reduces the footprint, cycles reactive energy between motor-connected submodules, and power-flow is easy to control. However, when the disadvantages of the MDC are in question, the transformer link is brought up again: it adds complexity, requires additional energy conversion stages, increases component count, adds losses, produces a load-dependent voltage drop, needs a flux and current balancing control, and is difficult to design with minimum and equal stray impedances.

Thus, the transformer link is an integral part in both the good and the bad of the MDC topology. Further studies on the improvement of its operation are encouraged. The following topics are given as examples of the details that the research on the transformer could include:

1. Transformer operating frequency was set at $18 \mathrm{kHz}$ throughout the simulations of this doctoral dissertation, because it was the designed operating frequency of the prototype transformer.

- Research should be conducted to find the operating frequency that provides the best compromise between the physical size and losses of the transformer and the losses of the H-bridge cells.

2. What are the best physical core geometry and winding configuration for a symmetric transformer?

- How does the inclusion of a seventh winding affect the design?

3 . Is it possible to apply the phase-shifting control, which is widely used in dual-activebridge DC/DC converters? The features of the phase-shifting control can be summarized as follows:

- Zero-voltage turn-off of the IGBTs reduces switching losses;

- Peak values of the current are considerably reduced;

- Active control of the phase shift provides regulation of the DC link voltages and eliminates the voltage drop of the transformer.

4. Replacing the multiwinding single-phase transformer with a high-frequency threephase transformer;

- The H-bridges apply the PWM strategy to produce phase voltages for the transformer.

5. An antisaturation control for the transformer was disclosed in (Korhonen et al., 2013b) and (Sankala et al., 2014a). Experimental validation of the control algorithm is highly recommended.

Finally, the matters of efficiency and reliability of the MDC are brought up. These two are major topics both of which were omitted from the dissertation. When it comes to the efficiency, a simple deduction can be performed: An H-bridge comprises four semiconductors. Two semiconductors carry the current at any given time instant, and the MDC includes $12 M$ $\mathrm{H}$-bridges. This means that there are $24 M$ conducting semiconductors at any given time instant. The same number for the CHB is $15 M$ or $12 M$ when a three-phase or single-phase input bridge configuration is applied, respectively. It is evident that the MDC exhibits higher conduction losses out of these topologies if the submodule voltage and current ratings are assumed equal. In addition, the transformer link generates high switching losses because a hard switching scheme is used. Without further calculation, it can be said that the efficiency of the MDC will very likely be lower than that of the CHB. However, if the switching losses 
at the transformer link can be mitigated for example by a phase-shift control, or by a seriesresonant capacitor, the efficiency may become comparable with the $\mathrm{CHB}$, since the MDC has no input transformer. Thus, further study on the efficiency is highly recommended.

Considering the reliability, excessive research was conducted on the topic in the UNIFLEXPM project. As the two topologies have an almost similar construction, the applicability of the research on the MDC should be investigated. After that, the details related to the reliability issue could be addressed. It is pointed out, however, that there are numerous factors that affect the reliability: component selection (passive and active), physical design of the power modules, and issues related to the manufacturing are but a few examples. Further, it is very difficult to assess these aspects at the topology concept level. 


\section{Chapter 6}

\section{Conclusion}

Multilevel converters have gained a strong foothold in medium-voltage motor drives in the industry over the past couple of decades. New power circuit topologies emerge frequently as research is conducted by the industry and academia. The development of new topologies is motivated by energy saving, striving for modular topologies, and intellectual property issues.

This dissertation work is a part of a university-industry collaboration research project. The industry partner's new innovative topology with the objectives presented above was in the focus of the project. The topology is known as the modular double-cascade converter (MDC), and it features two sets of cascaded H-bridge (CHB) inverters: one for the grid connection and the other for the load. The MDC is a highly modular topology constructed with low-voltage power modules. The number of modules is chosen according to the voltage and current rating of the application. The main results of the research project were presented in this doctoral dissertation.

The principle of converter operation and the development of the required control algorithms were presented. The MDC includes three separate interfaces, each with different control strategies. The first interface is the grid connection. The grid is interfaced with an LCL filter and a CHB rectifier. The design of the filter and the active front end controller was presented. The motor load is interfaced with a CHB inverter. Different connection principles; star, delta, and parallel connections, and multiport operation were analyzed. The third interface is a sixwinding medium-frequency transformer link, where each winding is supplied by an H-bridge inverter. The transformer is supplied by coherent rectangular voltages in an uncontrolled manner. The energy transfers through the isolation barrier based on the voltage difference of the submodules that are connected to the transformer.

Experimental results were obtained by a down-scaled prototype system. The prototype was constructed with commercial low-voltage inverters provided by the industry partner. The measurement results served the purpose of the proof-of-concept for the functionality of the MDC. 
The feasibility of the converter was studied by two approaches. In the first approach, the MDC was compared with parallel topologies. These other topologies were introduced in the literature review of the dissertation. The topologies were compared with regard to the component count and technical features with the focus on a special application in mining industry. It was discovered that the concept-level topology comparison is not sufficient to determine the most suitable topology for the application.

The second approach of the feasibility study focused on various technical details that affect the performance of the MDC. The study revealed both advantages and disadvantages of the MDC: The disadvantages were the limited application voltage rating resulting from the transformer maximum voltage blocking capability, poor performance in overloading conditions because of the voltage drop caused by the transformer, and the increasing magnitude of the $11^{\text {th }}$ and $13^{\text {th }}$ harmonics in grid currents when the number of cascaded power modules is increased. Considering the advantages, it was noticed that common-mode voltages are relatively low and the common-mode current has no return path, as it is blocked by the medium-frequency transformer in ideal case. However, the stray capacitances of the transformer create a path for the common-mode current.

At the beginning of the research project, it was anticipated that the topology could be constructed for commercial purposes with the existing low-voltage products. The research showed that this is not a practical approach because of the unequal current ratings of the different power modules and the complex medium-frequency transformer link. The modularity of the topology is further reduced by the nonmodular grid filter and the restriction on the number of cascaded cells. The latter restriction is due to the limited voltage blocking capability of the transformer.

While further development is needed to complete the features in the control algorithms, and especially in the transformer link, the efficiency and reliability of the MDC remain the most important future research subjects. 


\section{References}

ABB (2006), "MEGADRIVE-LCI, Medium voltage AC drive for control and soft starting of large synchronous motors," Tech. rep., ABB, available: www.abb.com.

ABB (2012), "Medium voltage AC drive ACS 2000, 250 - 2500 kW, 4.0 - 6.9 kV," Tech. rep., ABB, available: www.abb.com/drives.

Barbosa, P., Steimer, P., Steinke, J., Meysenc, L., Winkelnkemper, M., and Celanovic, N. (2005), "Active Neutral-Point-Clamped Multilevel Converters," in IEEE 36th Power Electronics Specialists Conference, 2005. PESC '05., pp. 2296-2301.

Bernet, S. and Brückner, T. (2003), "Open-loop and closed-loop control method for a threepoint converter with active clamped switches, and apparatus for this purpose," US Patent number: US2003165071A1.

Blasko, V. and Kaura, V. (1997), “A novel control to actively damp resonance in input LC filter of a three-phase voltage source converter," IEEE Transactions on Industry Applications, vol. 33, no. 2, pp. 542-550.

Carrara, G., Gardella, S., Marchesoni, M., Salutari, R., and Sciutto, G. (1992), “A new multilevel PWM method: a theoretical analysis," IEEE Transactions on Power Electronics, vol. 7, no. 3, pp. 497-505.

Chen, S., Lipo, T., and Fitzgerald, D. (1996), "Source of induction motor bearing currents caused by PWM inverters," IEEE Transactions on Energy Conversion, vol. 11, no. 1, pp. 25-32.

De Doncker, R., Divan, D., and Kheraluwala, M. (1991), “A three-phase soft-switched highpower-density DC/DC converter for high-power applications," IEEE Transactions on Industry Applications, vol. 27, no. 1, pp. 63-73.

Franquelo, L., Rodríguez, J., Leon, J., Kouro, S., Portillo, R., and Prats, M. (2008), “The age of multilevel converters arrives," IEEE Industrial Electronics Magazine, vol. 2, Issue 2, pp. 28-39.

Friedli, T. and Kolar, J. (2012), "Milestones in Matrix Converter Research,” IEEJ Journal of Industry Applications, vol. 1, no. 1, pp. 2-14.

Glinka, M. and Marquardt, R. (2005), “A new AC/AC multilevel converter family,” IEEE Transactions on Industrial Electronics, vol. 52, no. 3, pp. 662-669. 
Hammond, P. (1997), “A new approach to enhance power quality for medium voltage AC drives," IEEE Transactions on Industry Applications, vol. 33, no. 1, pp. 202-208.

Hiti, S., Vlatkovic, V., Borojevic, D., and Lee, F. (1994), “A new control algorithm for threephase PWM buck rectifier with input displacement factor compensation," IEEE Transactions on Power Electronics, vol. 9, no. 2, pp. 173-180.

Huang, H., Xu, A., and Xie, S. (2008), "Research on cascaded inverter with separated filters," in proceedings of 3rd IEEE Conference on Industrial Electronics and Applications, 2008. ICIEA 2008, pp. $2368-2372$.

Inoue, S. and Akagi, H. (2006), “A Bi-Directional Isolated DC/DC Converter as a Core Circuit of the Next-Generation Medium-Voltage Power Conversion System," in proceedings of 37th IEEE Power Electronics Specialists Conference, 2006. PESC '06, pp. 1-7.

Inoue, S. and Akagi, H. (2007), "Voltage Control of a Bi-Directional Isolated DC/DC Converter for Medium-Voltage Motor Drives," in proceedings of Power Conversion Conference - Nagoya, 2007. PCC '07, pp. 1244-1250.

Iov, F., Blaabjerg, F., Clare, J., Wheeler, P., Rufer, A., and Hyde, A. (2009), “UNIFLEX-PM - A Key-Enabling Technology for Future European Electricity Networks," EPE Journal, vol. 19 , no. 4 , pp. 6-16.

Itkonen, T. (2010), "Parallel-operation three-phase voltage source inverters - circulating current modeling, analysis and mitigation," Doctoral dissertation, Acta Universitatis Lappeenrantaensis 389 .

Kieferndorf, F., Basler, M., Serpa, L., Fabian, J.H., Coccia, A., and Scheuer, G. (2010), “A New Medium Voltage Drive System Based on ANPC-5L Technology," in proceedings of IEEE International Conference on Industrial Technology (ICIT), 2010, pp. 643-649.

Kieferndorf, F., Karamanakos, P., Bader, P., Oikonomou, N., and Geyer, T. (2012), "Model Predictive Control of the Internal Voltages of a Five-Level Active Neutral Point Clamped Converter," in proceedings of IEEE Energy Conversion Congress and Exposition (ECCE), 2012, pp. 1676-1683.

Komulainen, R. and Sarén, H. (2013), "Power transmission method and power transmission apparatus," U.S. Patent US 2013/8,553,432 B2.

Korhonen, J., Sankala, A., Ström, J.P., Luukko, J., Silventoinen, P., Komulainen, R., Sarén, H., Södö, N., and Isaksson, D. (2013a), "Power Direction Control of Medium Frequency Isolation DC/DC Converter for Modular Double Cascade Converter," in proceedings of 28th annual IEEE Applied Power Electronics Conference and Exposition (APEC), pp. 2240-2246.

Korhonen, J., Sankala, A., and Strandberg, S. (2013b), “Muuntajan ohjaus,” Finnish Patent, no. 123771 .

Kouro, S., Malinowski, M., Gopakumar, K., Pou, J., Franquelo, L., Wu, B., Rodriguez, J., Perez, M., and Leon, J. (2010), "Recent Advances and Industrial Applications of Multilevel Converters," IEEE Transactions on Industrial Electronics, vol. 57, no. 8, pp. 2553-2580. 
Laakkonen, T. (2010), "Distributed Control Architecture of Power Electronics BuildingBlock-Based Frequency Converters," Doctoral dissertation, Acta Universitatis Lappeenrantaensis 403 .

Lezana, P., Rodriguez, J., Perez, M., and Espinoza, J. (2009), "Input Current Harmonics in a Regenerative Multicell Inverter With Single-Phase PWM Rectifiers," IEEE Transactions on Industrial Electronics, vol. 56, no. 2, pp. 408-417.

Liserre, M., Blaabjerg, F., and Hansen, S. (2005), "Design and control of an LCL-filter-based three-phase active rectifier," IEEE Transactions on Industry Applications, vol. 41, no. 5, pp. 1281-1291.

Magro, M.C. and Savio, S. (2009), "Dependability and Impact Analysis for a Universal and Flexible Power Management System,” EPE Journal, vol. 19, no. 4, pp. 51-58.

McGrath, B. and Holmes, D. (2002), "Multicarrier PWM strategies for multilevel inverters," IEEE Transactions on Industrial Electronics, vol. 49, no. 4, pp. 858-867.

McMurray, W. (1971), "Fast response stepped-wave switching power converter circuit," United States Patent, no. 3581212.

Meynard, T. and Foch, H. (1993), "Dispositif électronique de conversion d'énergie électrique," French Patent, no. 267971 5B1.

Mohan, N., Undeland, T.M., and Robbins, W.P. (2003), Power Electronics Converters, Applications, and Design, John Wiley \& Sons Inc., third edn.

Nabae, A., Takahashi, I., and Akagi, H. (1981), "A New Neutral-Point-Clamped PWM Inverter," IEEE Transactions on Industry Applications, vol. IA-17, no. 5, pp. 518-523.

Najafi, E. and Yatim, A. (2012), "Design and Implementation of a New Multilevel Inverter Topology," IEEE Transactions on Industrial Electronics, vol. 59, no. 11, pp. 4148-4154.

Naumanen, V. (2010), "Multilevel Converter Modulation: Implementation and Analysis," Doctoral dissertation, Acta Universitatis Lappeenrantaensis 386.

Neft, C. and Schauder, C.D. (1992), "Theory and design of a 30-hp matrix converter," IEEE Transactions on Industry Applications, vol. 28, no. 3, pp. 546-551.

Ortiz, G., Faessler, L., Kolar, J., and Apeldoorn, O. (2014), "Flux Balancing of Isolation Transformers and Application of "The Magnetic Ear" for Closed-Loop Volt-Second Compensation," IEEE Transactions on Power Electronics, vol. 29, pp. 4078-4090.

Ortiz, G., Muhlethaler, J., and Kolar, J. (2011), “"Magnetic Ear"-based balancing of magnetic flux in high power medium frequency dual active bridge converter transformer cores," in Proceedings of IEEE 8th International Conference on Power Electronics and ECCE Asia (ICPE ECCE), pp. 1307-1314.

Patel, H.S. and Hoft, R.G. (1973), "Generalized Techniques of Harmonic Elimination and Voltage Control in Thyristor Inverters: Part I-Harmonic Elimination," IEEE Transactions on Industry Applications, vol. IA-9, no. 3, pp. 310-317. 
Petersson, T. and Frank, K. (1972), "Starting of Large Synchronous Motor Using Static Frequency Converter," IEEE Transactions on Power Apparatus and Systems, vol. PAS-91, no. 1 , pp. 172-179.

Phillips, K.P. (1972), “Current-Source Converter for AC Motor Drives," IEEE Transactions on Industry Applications, vol. IA-8, no. 6, pp. 679-683.

Rockhill, A., Liserre, M., Teodorescu, R., and Rodriguez, P. (2011), "Grid-Filter Design for a Multimegawatt Medium-Voltage Voltage-Source Inverter," IEEE Transactions on Industrial Electronics, vol. 58, no. 4, pp. 1205-1217.

Rodríguez, J., Franquelo, L., Kouro, S., Leon, J., Portillo, R., Prats, M., and Perez, M. (2009), "Multilevel Converters: An Enabling Technology for High-Power Applications," Proceedings of the IEEE, vol. 97, no. 11, pp. 1786-1817.

Rodríguez, J., Lai, J.S., and Peng, F.Z. (2002), "Multilevel inverters: a survey of topologies, controls, and applications," IEEE Transactions on Industrial Electronics, vol. 49, no. 4, pp. 724-738.

Rodriguez, J., Pontt, J., Alzarnora, G., Becker, N., Einenkel, O., and Weinstein, A. (2002a), "Novel 20-MW Downhill Conveyor System Using Three-Level Converters," IEEE Transactions on Industrial Electronics, vol. 49, no. 5, pp. 1093-1100.

Rodriguez, J., Pontt, J., Becker, N., and Weinstein, A. (2002b), "Regenerative Drives in the Megawatt Range for High-Performance Downhill Belt Conveyors," IEEE Transactions on Industry Applications, vol. 38, no. 1, pp. 203-210.

Sankala, A., Korhonen, J., Hannonen, J., Ström, J.P., Silventoinen, P., Komulainen, R., Sarén, H., Strandberg, S., and Södö, N. (2014a), "Flux and Winding Current Balancing Control for a Medium-Frequency Six-Winding Transformer," in proceedings of 40th Annual Conference of the IEEE Industrial Electronics Society (IECON), pp. 1473-1479.

Sankala, A., Korhonen, J., Purhonen, M., Ström, J.P., Silventoinen, P., Komulainen, R., Sarén, H., Södö, N., and Strandberg, S. (2014b), "Design of an Active Front End for a Modular Double-Cascade converter," in proceedings of 16th European Conference on Power Electronics and Applications, EPE'14 ECCE Europe, pp. 1-10.

Sankala, A., Korhonen, J., Ström, J.P., Luukko, J., Silventoinen, P., Komulainen, R., Sarén, H., Södö, N., and Isaksson, D. (2012), "Modular Double-Cascade Converter," in proceedings of 27th annual IEEE Applied Power Electronics Conference and Exposition (APEC), pp. 647-652.

Sankala, A., Korhonen, J., Ström, J.P., Luukko, J., Silventoinen, P., Komulainen, R., Sarén, H., Södö, N., and Isaksson, D. (2015), "Modular Double-Cascade Converter for HighPower Medium-Voltage Drives," IET Power Electronics, accepted for publication on March 26th 2015.

Sankala, A., Korhonen, J., Ström, J.P., Silventoinen, P., Komulainen, R., Sarén, H., Södö, N., and Dilley, D. (2013), "Modular Double-Cascade converter with soft switching DC/DC isolation converter," in proceedings of 15th European Conference on Power Electronics and Applications, EPE'13 ECCE Europe, pp. 1-9. 
Siemaszko, D., Zurkinden, F., Fleischli, L., Villar, I., Novaes, Y.R.D., and Rufer., A. (2009), "Description and Efficiency Comparison of Two $25 \mathrm{kVA}$ DC/AC Isolation Modules," EPE Journal, vol. 19, no. 4, pp. 17-24.

Spichartz, M., Staudt, V., and Steimel, A. (2013), "Modular Multilevel Converter for propulsion system of electric ships," in proceedings of IEEE Electric Ship Technologies Sympo$\operatorname{sium}(E S T S), 2013$, pp. 237-242.

UNIFLEX-PM (2007), "Report on converter structures - UNIFLEX-PM project deliverable D3.1," Tech. rep., University of Nottingham, availabe: http://www.eee.nottingham.ac.uk/uniflex/.

Watson, A., Wheeler, P., and Clare, J. (2007), “A selective harmonic elimination system for restoring and equalising DC link voltages in a multilevel active rectifier," in proceedings of European Conference on Power Electronics and Applications, 2007, pp. 1-7.

Wu, B., Pontt, J., Rodriguez, J., Bernet, S., and Kouro, S. (2008), “Current-Source Converter and Cycloconverter Topologies for Industrial Medium-Voltage Drives," IEEE Transactions on Industrial Electronics, vol. 55, no. 7, pp. 2786-2797.

Yaskawa (2008), "Super Energy-Saving Medium-Voltage Matrix Converter with Power Regeneration FSDrive-MX1S," Tech. Rep. LITERATURE NO. KAEP C710688 00C, Yaskawa Electric Corporation, available: www.yatec.com. 
Appendices 


\section{Appendix A}

\section{Simulation model}

The simulation model in the Matlab Simulink environment is presented in this appendix. The model comprises a three-phase grid, an LCL filter, one MDC belt, an asynchronous induction machine model, all related controllers, and a signal storage for offline data analysis.

The model has a hierarchical structure, and only the first two levels of the hierarchy are shown. The details of the lower levels are delineated in the body text of the dissertation.

For illustrative purposes, the model was divided into seven parts, each of which is presented in Figures A.1-A.7.

The first figure outlines the electrical connections on the grid side of the belt. The grid and the LCL filter are lumped inside one block. The belt is connected in a wye configuration on both the grid and motor sides. A phase voltage and current measurement block is located between the submodules and the transformer. The second figure shows the corresponding connections on the motor side of the transformer. In the cases of multiple belts, the belt construction shown here is copied and pasted, and the required connections are made.

The third and fourth figures show the controllers and modulators for the grid and motor interfaces, respectively. The functionality of the lower hierarchy levels is presented in the body text. The modulators were individually constructed for each number of belts.

The fifth figure presents the transformer modulator. Each transformer-connected H-bridge is commanded by the same gate instructions. The figure also shows the blocks used for signal storage for the offline data analysis.

The sixth figure depicts the components used to build each submodule. The transistors are IGBT/diode blocks provided by the Simscape/SimpowerSystems toolbox. The block implements an ideal IGBT and an antiparallel diode. The parameters of the IGBT block used in the simulations are: an internal resistance of $1 \mathrm{~m} \Omega$, a snubber resistance of $100 \mathrm{k} \Omega$, and an 
infinite snubber capacitance.

The final figure shows the models of the grid and the LCL filter. Phase voltages are generated by three AC voltage sources connected in series with an inductor and a resistor. Both the inductors and the capacitor of the LCL have series resistors. The block outputs four threephase measurements. The grid voltage measurements include the voltage drop produced by the grid impedance. Inverter-side voltages are measured against the neutral point of the grid inverter, which is routed to the block from outside.

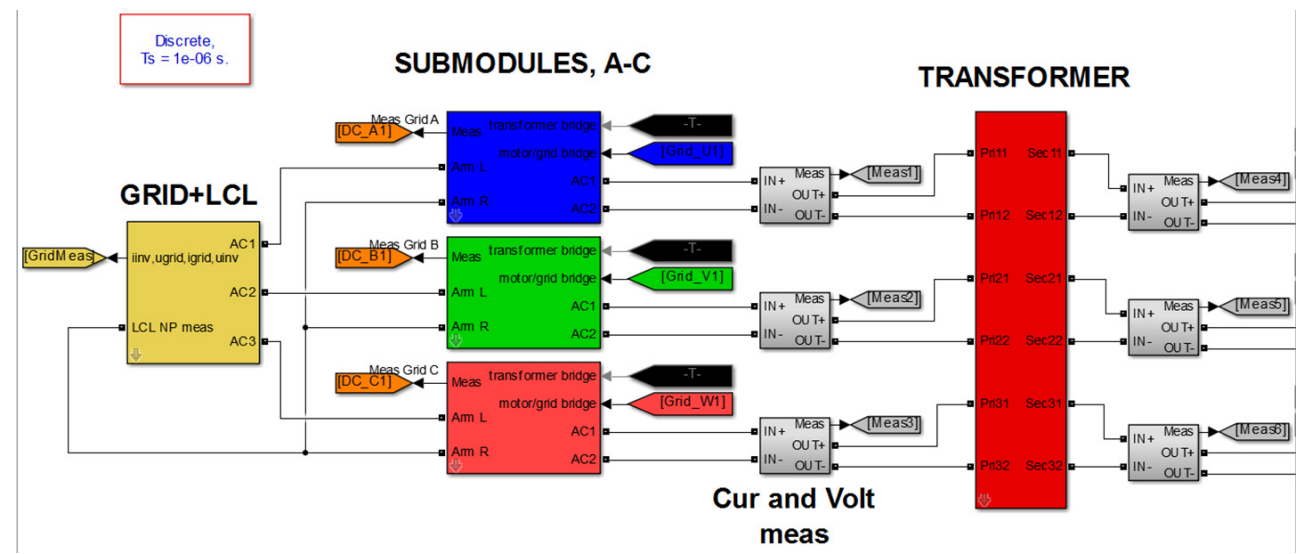

Figure A.1. Simulation model Part 1: Top-level connections of the grid+LCL, the submodules on the grid side and the transformer.

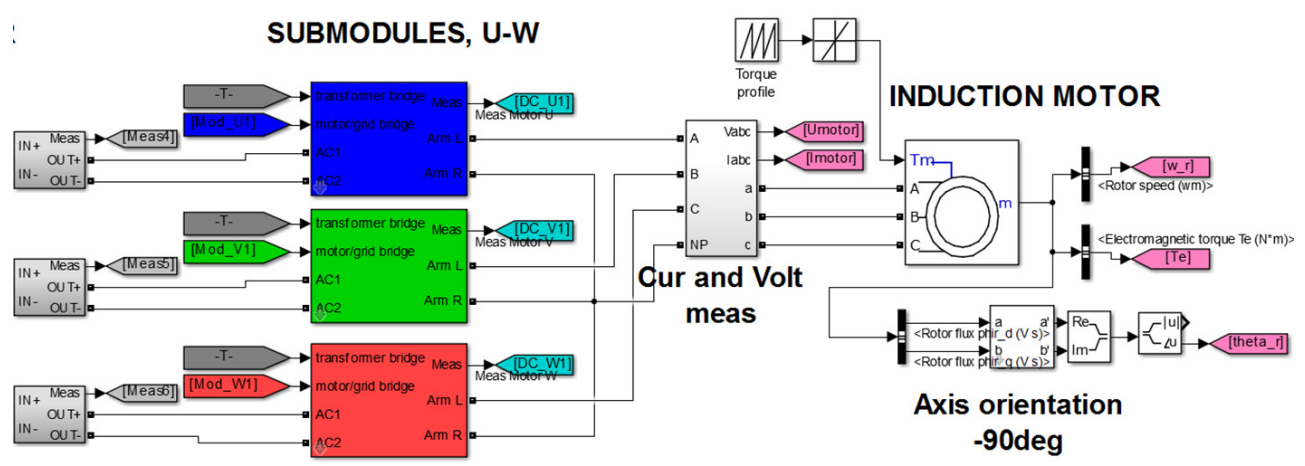

Figure A.2. Simulation model Part 2: Top-level connections of the transformer, the submodules on the motor side and the motor. 


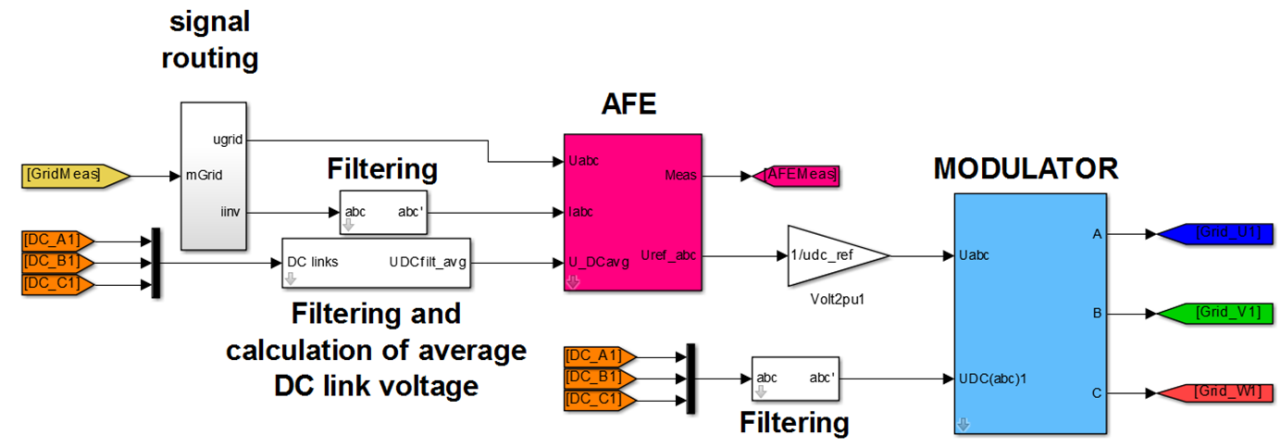

Figure A.3. Simulation model Part 3: Top-level, the AFE and the grid bridge modulator.

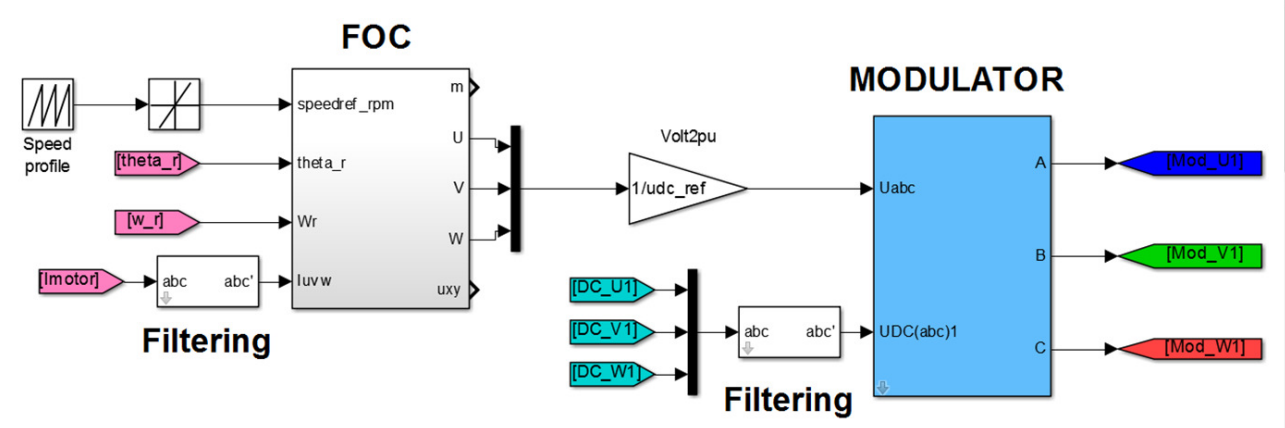

Figure A.4. Simulation model Part 4: Top-level, the FOC and the motor bridge modulator.

TRANSFORMER MODULATOR

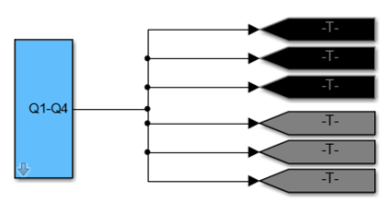

Storage simulation results to .mat files

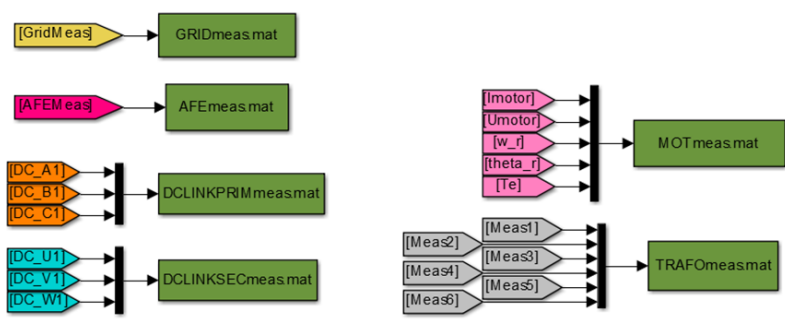

Figure A.5. Simulation model Part 5: Top-level, the transformer bridge modulator and the signal storage for offline processing. 


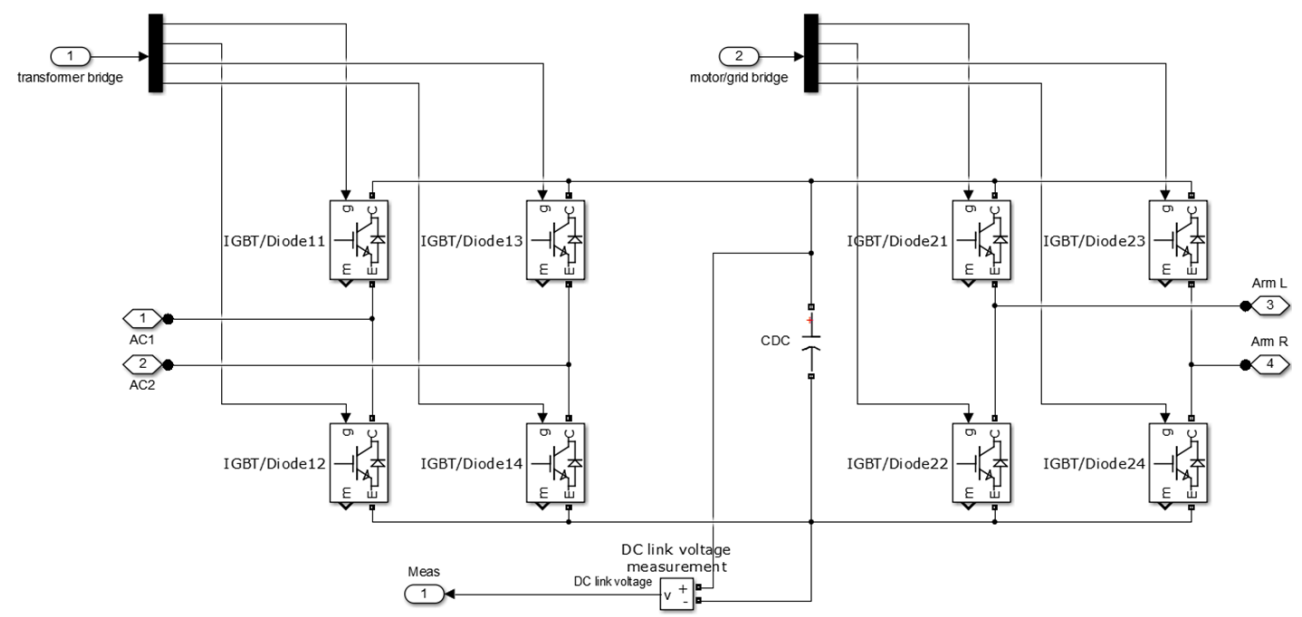

Figure A.6. Simulation model Part 6: Submodule block: Two IGBT/diode H-bridges connected backto-back with an intermediate DC link capacitor.

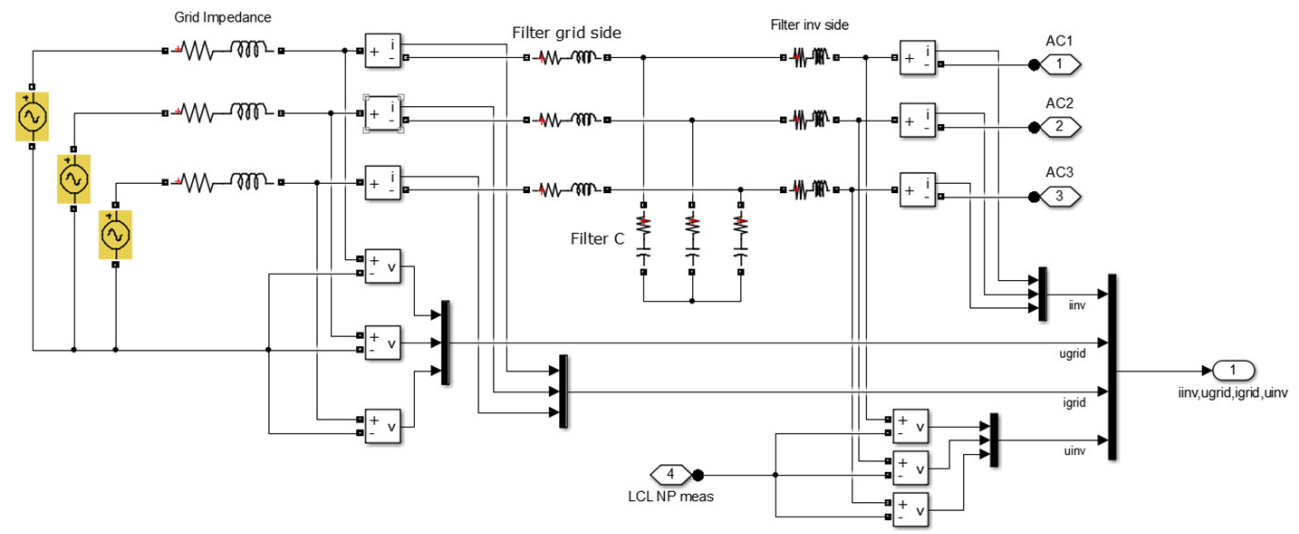

Figure A.7. Simulation model Part 7: Grid+LCL block. 


\section{Appendix B}

\section{Simulation parameters}

The parameters used in the simulation model of the MDC are described in this appendix. The simulations are performed for systems with 1,2,3,4, and 5 cascaded belts on the grid side. In most of the simulations, the load-side belts are also cascaded with the only exception being the simulations of section 3.5 .

The parameters that are common for each simulation are listed in Table B.1. Different grid impedances are used in the simulations with different power levels. The short-circuit to nominal current ratio has been kept constant at 100. The grid inductance and resistance values are presented in Table B.2.

The LCL filter and the AFE controller are designed individually for each power level using the guidelines presented in sections 3.4.1 and 3.4.2, respectively. The numerical values of the filter and controller parameters are given in Tables B.3 and B.4 for the simulations where the apparent switching frequency of the grid inverter is $6.0 \mathrm{kHz}$ regardless of the number of belts. On the other hand, Tables B.5 and B.6 present the filter and controller parameters for the simulations where the switching frequency of a single IGBT is $1.0 \mathrm{kHz}$, and the apparent switching frequency increases with the number of cascaded belts $M$. The cross coupling factor $k_{\mathrm{cc}}$ is calculated individually for each system by

$$
k_{\mathrm{cc}}=\omega_{\text {grid }} L_{\mathrm{tot}}=2 \pi f_{\text {grid }}\left[L_{\mathrm{g}}\left(I_{\text {ratio }}=50\right)+L_{1}+L_{2}\right] .
$$


Table B.1. Simulation parameters: Common for all simulations

\begin{tabular}{llcl}
\hline Element & Symbol & Value & Unit \\
\hline Grid frequency & $f_{\text {grid }}$ & 50 & $\mathrm{~Hz}$ \\
Nominal active power of a belt & $P_{\text {belt }}$ & 750 & $\mathrm{~kW}$ \\
Submodule DC link capacitance & $C_{\mathrm{dc}}$ & 1000 & $\mu \mathrm{F}$ \\
Simulation time step & $t_{\mathrm{s}}$ & 1.0 & $\mu \mathrm{s}$ \\
Phase leg dead time in every H-bridge & $t_{\text {dead }}$ & 2.0 & $\mu \mathrm{s}$ \\
Transformer operating frequency & $f_{\text {sw,trafo }}$ & 18.0 & $\mathrm{kHz}$ \\
Apparent switching frequency, motor & $f_{\text {sw,app,mot }}$ & 6.0 & $\mathrm{kHz}$ \\
Short-circuit to nominal current ratio & $I_{\text {ratio }}$ & 100 & - \\
\hline
\end{tabular}

Table B.2. Simulation parameters: Grid

\begin{tabular}{cccc}
\hline$M$ & $U_{\mathrm{LL}}$ & $L_{\mathrm{g}}$ & $R_{\mathrm{g}}$ \\
\hline 1 & $1100 \mathrm{~V}$ & $50.3 \mu \mathrm{H}$ & $3.20 \mathrm{~m} \Omega$ \\
2 & $2300 \mathrm{~V}$ & $110 \mu \mathrm{H}$ & $7.10 \mathrm{~m} \Omega$ \\
3 & $3300 \mathrm{~V}$ & $151 \mu \mathrm{H}$ & $9.70 \mathrm{~m} \Omega$ \\
4 & $4160 \mathrm{~V}$ & $180 \mu \mathrm{H}$ & $11.5 \mathrm{~m} \Omega$ \\
5 & $5700 \mathrm{~V}$ & $270 \mu \mathrm{H}$ & $17.3 \mathrm{~m} \Omega$ \\
\hline
\end{tabular}

Table B.3. Simulation parameters: LCL, $f_{\mathrm{sw}, \text { app, grid }}=6.0 \mathrm{kHz}$

\begin{tabular}{ccccccc}
\hline$M$ & $L_{1}$ & $R_{1}$ & $C_{\mathrm{f}}$ & $R_{\mathrm{d}}$ & $L_{2}$ & $R_{2}$ \\
\hline 1 & $412 \mu \mathrm{H}$ & $8.10 \mathrm{~m} \Omega$ & $19.7 \mu \mathrm{F}$ & $0.70 \Omega$ & $230 \mu \mathrm{H}$ & $8.10 \mathrm{~m} \Omega$ \\
2 & $430 \mu \mathrm{H}$ & $17.6 \mathrm{~m} \Omega$ & $42.4 \mu \mathrm{F}$ & $0.70 \Omega$ & $92.3 \mu \mathrm{H}$ & $17.6 \mathrm{~m} \Omega$ \\
3 & $412 \mu \mathrm{H}$ & $24.2 \mathrm{~m} \Omega$ & $26.3 \mu \mathrm{F}$ & $0.70 \Omega$ & $158 \mu \mathrm{H}$ & $24.2 \mathrm{~m} \Omega$ \\
4 & $372 \mu \mathrm{H}$ & $28.8 \mathrm{~m} \Omega$ & $25.9 \mu \mathrm{F}$ & $0.70 \Omega$ & $157 \mu \mathrm{H}$ & $28.8 \mathrm{~m} \Omega$ \\
5 & $427 \mu \mathrm{H}$ & $43.3 \mathrm{~m} \Omega$ & $17.3 \mu \mathrm{F}$ & $0.70 \Omega$ & $242 \mu \mathrm{H}$ & $43.3 \mathrm{~m} \Omega$ \\
\hline
\end{tabular}

Table B.4. Simulation parameters: AFE, $f_{\mathrm{sw}, \text { app,grid }}=6.0 \mathrm{kHz}$

\begin{tabular}{ccccc}
\hline$M$ & $K_{\mathrm{P}, \mathrm{CC}}$ & $K_{\mathrm{I}, \mathrm{CC}}$ & $K_{\mathrm{P}, \mathrm{VC}}$ & $K_{\mathrm{I}, \mathrm{VC}}$ \\
\hline 1 & 1.48 & 98.6 & 4.50 & 929.8 \\
2 & 1.49 & 98.6 & 1.70 & 175.6 \\
3 & 1.69 & 136 & 1.70 & 128.5 \\
4 & 1.78 & 161 & 1.70 & 96.4 \\
5 & 2.42 & 243 & 1.70 & 70.25 \\
\hline
\end{tabular}


Table B.5. Simulation parameters: LCL, $f_{\mathrm{sw}, \mathrm{app}, \text { grid }}=2 M f_{\mathrm{sw}, \mathrm{IGBT}}=2 M \times 1.0 \mathrm{kHz}$

\begin{tabular}{ccccccc}
\hline$M$ & $L_{1}$ & $R_{1}$ & $C_{\mathrm{f}}$ & $R_{\mathrm{d}}$ & $L_{2}$ & $R_{2}$ \\
\hline 1 & $1235 \mu \mathrm{H}$ & $8.07 \mathrm{~m} \Omega$ & $78.9 \mu \mathrm{F}$ & $0.70 \Omega$ & $514 \mu \mathrm{H}$ & $8.07 \mathrm{~m} \Omega$ \\
2 & $646 \mu \mathrm{H}$ & $17.6 \mathrm{~m} \Omega$ & $36.1 \mu \mathrm{F}$ & $0.70 \Omega$ & $273 \mu \mathrm{H}$ & $17.6 \mathrm{~m} \Omega$ \\
3 & $412 \mu \mathrm{H}$ & $24.2 \mathrm{~m} \Omega$ & $26.3 \mu \mathrm{F}$ & $0.70 \Omega$ & $158 \mu \mathrm{H}$ & $24.2 \mathrm{~m} \Omega$ \\
4 & $279 \mu \mathrm{H}$ & $28.8 \mathrm{~m} \Omega$ & $16.6 \mu \mathrm{F}$ & $0.70 \Omega$ & $138 \mu \mathrm{H}$ & $28.8 \mathrm{~m} \Omega$ \\
5 & $256 \mu \mathrm{H}$ & $43.3 \mathrm{~m} \Omega$ & $11.0 \mu \mathrm{F}$ & $0.70 \Omega$ & $124 \mu \mathrm{H}$ & $43.3 \mathrm{~m} \Omega$ \\
\hline
\end{tabular}

Table B.6. Simulation parameters: AFE, $f_{\mathrm{sw}, \text { app,grid }}=2 M f_{\mathrm{sw}, \mathrm{IGBT}}=2 M \times 1.0 \mathrm{kHz}$

\begin{tabular}{ccccc}
\hline$M$ & $K_{\mathrm{P}, \mathrm{CC}}$ & $K_{\mathrm{I}, \mathrm{CC}}$ & $K_{\mathrm{P}, \mathrm{VC}}$ & $K_{\mathrm{I}, \mathrm{VC}}$ \\
\hline 1 & 1.23 & 15.1 & 0.30 & 15.50 \\
2 & 1.52 & 65.8 & 2.50 & 258.3 \\
3 & 1.69 & 136 & 1.70 & 128.5 \\
4 & 2.07 & 215 & 4.50 & 255.1 \\
5 & 3.07 & 404 & 4.50 & 186.0 \\
\hline
\end{tabular}




\section{ACTA UNIVERSITATIS LAPPEENRANTAENSIS}

597. POLIKARPOVA, MARIIA.Liquid cooling solutions for rotating permanent magnet synchronous machines. 2014. Diss.

598. CHAUDHARI, ASHVINKUMAR. Large-eddy simulation of wind flows over complex terrains for wind energy applications. 2014. Diss.

599. PURHONEN, MIKKO. Minimizing circulating current in parallel-connected photovoltaic inverters. 2014. Diss.

600. SAUKKONEN, ESA. Effects of the partial removal of wood hemicelluloses on the properties of kraft pulp. 2014. Diss.

601. GUDARZI, DAVOOD. Catalytic direct synthesis of hydrogen peroxide in a novel microstructured reactor. 2014. Diss.

602. VALKEAPÄÄ, ANTTI. Development of finite elements for analysis of biomechanical structures using flexible multibody formulations. 2014. Diss.

603. SSEBUGERE, PATRICK. Persistent organic pollutants in sediments and fish from Lake Victoria, East Africa. 2014. Diss.

604. STOKLASA, JAN. Linguistic models for decision support. 2014. Diss.

605. VEPSÄLÄINEN, ARI. Heterogenous mass transfer in fluidized beds by computational fluid dynamics. 2014. Diss.

606. JUVONEN, PASI. Learning information technology business in a changing industry landscape. The case of introducing team entreneurship in renewing bachelor education in information technology in a university of applied sciences. 2014. Diss.

607. MÄKIMATTILA, MARTTI. Organizing for systemic innovations - research on knowledge, interaction and organizational interdependencies. 2014. Diss.

608. HÄMÄLÄINEN, KIMMO. Improving the usability of extruded wood-plastic composites by using modification technology. 2014. Diss.

609. PIRTTILÄ, MIIA. The cycle times of working capital: financial value chain analysis method. 2014. Diss.

610. SUIKKANEN, HEIKKI. Application and development of numerical methods for the modelling of innovative gas cooled fission reactors. 2014. Diss.

611. LI, MING. Stiffness based trajectory planning and feedforward based vibration suppression control of parallel robot machines. 2014. Diss.

612. KOKKONEN, KIRSI. From entrepreneurial opportunities to successful business networks - evidence from bioenergy. 2014. Diss.

613. MAIJANEN-KYLÄHEIKO, PÄIVI. Pursuit of change versus organizational inertia: a study on strategic renewal in the Finnish broadcasting company. 2014. Diss.

614. MBALAWATA, ISAMBI SAILON. Adaptive Markov chain Monte Carlo and Bayesian filtering for state space models. 2014. Diss.

615. UUSITALO, ANTTI. Working fluid selection and design of small-scale waste heat recovery systems based on organic rankine cycles. 2014. Diss. 
616. METSO, SARI. A multimethod examination of contributors to successful on-the-job learning of vocational students. 2014. Diss.

617. SIITONEN, JANI. Advanced analysis and design methods for preparative chromatographic separation processes. 2014. Diss.

618. VIHAVAINEN, JUHANI. VVER-440 thermal hydraulics as computer code validation challenge. 2014. Diss.

619. AHONEN, PASI. Between memory and strategy: media discourse analysis of an industrial shutdown. 2014. Diss.

620. MWANGA, GASPER GODSON. Mathematical modeling and optimal control of malaria. 2014. Diss.

621. PELTOLA, PETTERI. Analysis and modelling of chemical looping combustion process with and without oxygen uncoupling. 2014. Diss.

622. NISKANEN, VILLE. Radio-frequency-based measurement methods for bearing current analysis in induction motors. 2014. Diss.

623. HYVÄRINEN, MARKO. Ultraviolet light protection and weathering properties of woodpolypropylene composites. 2014. Diss.

624. RANTANEN, NOORA. The family as a collective owner - identifying performance factors in listed companies. 2014. Diss.

625. VÄNSKÄ, MIKKO. Defining the keyhole modes - the effects on the molten pool behavior and the weld geometry in high power laser welding of stainless steels. 2014. Diss.

626. KORPELA, KARI. Value of information logistics integration in digital business ecosystem. 2014. Diss.

627. GRUDINSCHI, DANIELA. Strategic management of value networks: how to create value in cross-sector collaboration and partnerships. 2014. Diss.

628. SKLYAROVA, ANASTASIA. Hyperfine interactions in the new Fe-based superconducting structures and related magnetic phases. 2015. Diss.

629. SEMKEN, R. SCOTT. Lightweight, liquid-cooled, direct-drive generator for high-power wind turbines: motivation, concept, and performance. 2015. Diss.

630. LUOSTARINEN, LAURI. Novel virtual environment and real-time simulation based methods for improving life-cycle efficiency of non-road mobile machinery. 2015. Diss.

631. ERKKILÄ, ANNA-LEENA. Hygro-elasto-plastic behavior of planar orthotropic material. 2015. Diss.

632. KOLOSENI, DAVID. Differential evolution based classification with pool of distances and aggregation operators. 2015. Diss.

633. KARVONEN, VESA. Identification of characteristics for successful university-company partnership development. 2015. Diss.

634. KIVYIRO, PENDO. Foreign direct investment, clean development mechanism, and environmental management: a case of Sub-Saharan Africa. 2015. Diss. 
